\title{
Paramedics' perceptions of caring for those who self-harm: A Qualitative Study
}

\author{
Rees, Nigel
}

How to cite:

Rees, Nigel (2019) Paramedics' perceptions of caring for those who self-harm: A Qualitative Study. Doctoral thesis, Swansea University.

http://cronfa.swan.ac.uk/Record/cronfa51912

Use policy:

This item is brought to you by Swansea University. Any person downloading material is agreeing to abide by the terms of the repository licence: copies of full text items may be used or reproduced in any format or medium, without prior permission for personal research or study, educational or non-commercial purposes only. The copyright for any work remains with the original author unless otherwise specified. The full-text must not be sold in any format or medium without the formal permission of the copyright holder. Permission for multiple reproductions should be obtained from the original author.

Authors are personally responsible for adhering to copyright and publisher restrictions when uploading content to the repository.

Please link to the metadata record in the Swansea University repository, Cronfa (link given in the citation reference above.)

http://www.swansea.ac.uk/library/researchsupport/ris-support/ 


\title{
Paramedics' perceptions of caring for those who self-harm: A Qualitative Study
}

Nigel Rees QAM, FCPara, BSc (Hons), MSc,

\author{
Submitted to Swansea University \\ in fulfilment of the requirements of the degree of \\ Doctor of Philosophy $(\mathrm{PhD})$ \\ Swansea University
}

December 2018

Primary Supervisor: Associate Professor Alison Porter

Associate Supervisor: Professor Anne John

Associate Supervisor: Professor Frances Rapport 


\section{Summary}

\section{Background}

Self-harm (SH) accounts for over 5\% of the workload of emergency ambulance services and therefore paramedics are often the first health professional in contact with people who SH. This study aimed to explore paramedics' perceptions of caring for those who self-harm in order to inform education policy and practice.

\section{Methods}

A systematic review of quantitative literature and metasynthesis of the qualitative literature were conducted and informed the study proper, which took place between 2014-2016 in one UK ambulance service covering a population of three million people. Semi structured interviews were conducted, purposively sampling paramedics until saturation was reached. Interviews were recorded, transcribed verbatim, and coded through open, axial and selective levels of coding, identifying the Basic Social Process (BSP) and developing a Grounded Theory. A second researcher $(\mathrm{SH})$ independently reviewed early results, which were also memberchecked with participants.

\section{Results}

Eleven paramedics were interviewed. The following six categories emerged: Context; Judgements and values; Isolation and system failure; Managing risk; Competence at the boundary of mental and physical health needs; Professional, legal and ethical tensions. The BSP decision making in a context of risk was identified. The final Grounded Theory that emerged was one of 'Wicked Complexity of paramedic care for people who $\mathrm{SH}$, which includes usual factors such as tiredness and frequent callers, heightened factors including lack of support and pathways, and factors specific to $\mathrm{SH}$ such assessing mental health and suicide risk.

\section{Conclusions}

The study reported in this thesis builds on a very small body of literature to have explored paramedic care for people who $\mathrm{SH}$ and has found that this care interaction provides uniquely complex challenges. The multiple influences within the categories defined in this study need considering conjointly when making improvements to care. 


\section{Declaration}

This work has not previously been accepted in substance for any degree and is not being concurrently submitted in candidature for any degree.

Signed

Date

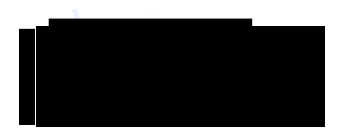

$29 / 07 / 2019$

\section{Statement 1}

This thesis is the result of my own investigations, except where otherwise stated. Where correction services have been used, the extent and nature of the correction is clearly marked in a footnote(s).

Other sources are acknowledged by giving explicit references. A bibliography of references is appended.

Signed (candidate)

Date 29/07/2019

\section{Statement 2}

I hereby give consent for my thesis, if accepted, to be available online in the University's Open Access Repository and for inter-library loan, and for the title and summary to be made available to outside organisations.

Signed

(candidate)

Date 29/07/2019 


\section{Table of Contents}

$\begin{array}{llllllllllll}\text { Summary } & . . & . . & . . & . & . & . & . . & . . & . . & . . & 2\end{array}$

$\begin{array}{lllllllllll}\text { Declarations and Statements } & . . & . . & . . & . . & . . & . . & 3\end{array}$

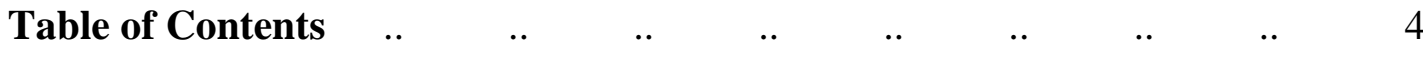

$\begin{array}{llllllllllllll}\text { List of Figures } & . . & . . & . . & . . & . & . & . & . & & . & & & \end{array}$

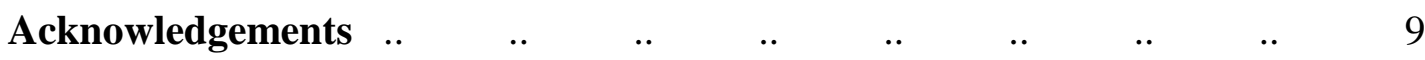

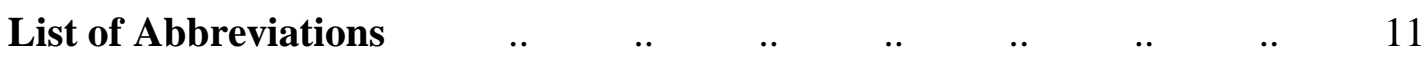

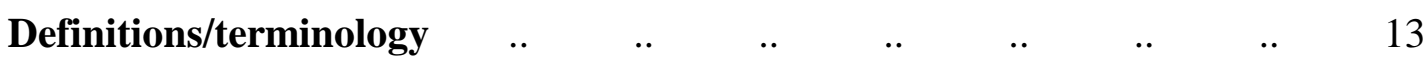

$\begin{array}{llllllllll}\text { Chapter One - Introduction } & . . & . . & . & \text {. } & \text {. } & \text {. } & \text {.. } & 19\end{array}$

$\begin{array}{lllllllll}\text { Research aims and objectives } & . . & . . & . . & . . & . . & . . & 19\end{array}$

$\begin{array}{lllllllllll}\text { Background } & . . & . . & . . & . & . & . & . . & . . & . . & 20\end{array}$

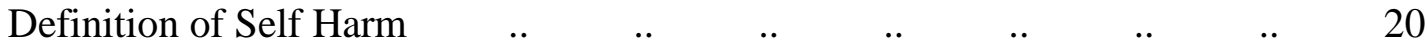

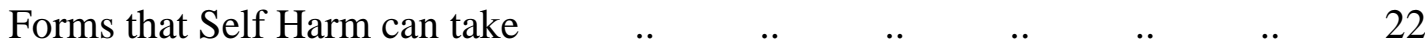

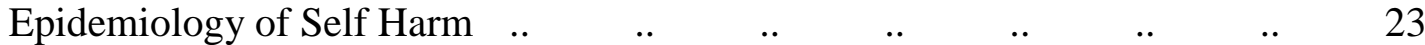

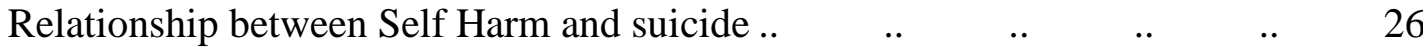

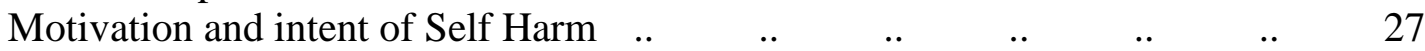

Experience of the care interaction between health professionals and people who

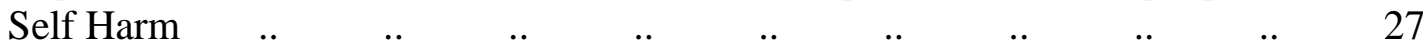

Role of police and detention in the care of people who Self Harm $\quad . . \quad 3 \quad$.. 30

$\begin{array}{lllllllllll}\text { Overview of thesis } & . . & . . & . . & . . & . . & . . & . . & . . & 32\end{array}$

Chapter Two - Context of paramedic care for those who Self harm

$\begin{array}{lllllllllll}\text { Introduction } & . . & . . & . . & . . & . . & . . & . . & . . & . . & 33\end{array}$

Current prehospital care provision: within the United Kingdom and

$\begin{array}{lllllllllll}\text { Internationally .. } & . . & . . & . . & . & . & . . & . . & . . & 33\end{array}$

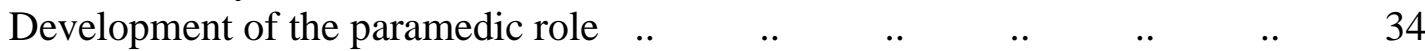

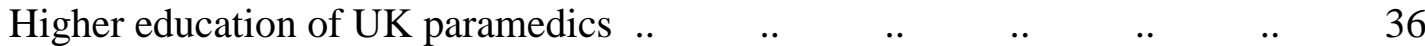

Move towards clinical effectiveness, governance, and evidence-based care in

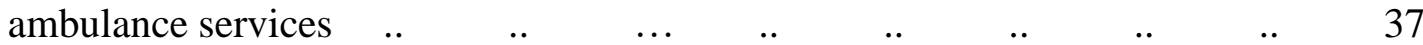

$\begin{array}{llllllllll}\text { Ambulance services as clinical providers of care } & . & . & . & & . & & . & & 38\end{array}$

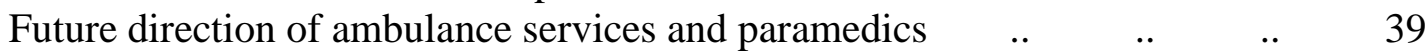

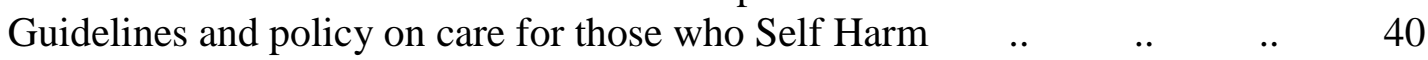

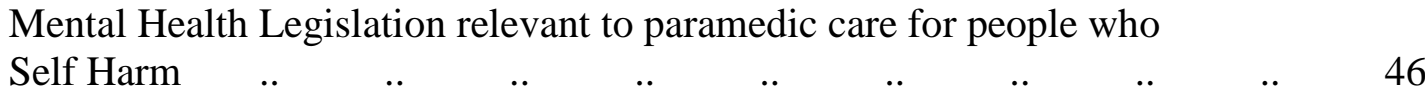

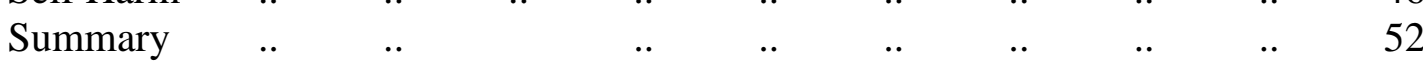

Chapter Three - Research Design: Methodology and methods

$\begin{array}{lllllllllll}\text { Introduction } & . . & . . & . & . . & . . & . . & . . & . . & . . & 56\end{array}$

Literature review and its place in Evolved Grounded Theory Methodology (EGTM)

Grounded Theory Methodology (GTM)

Evolution of GTM 


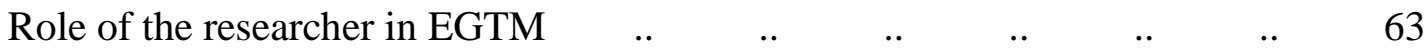

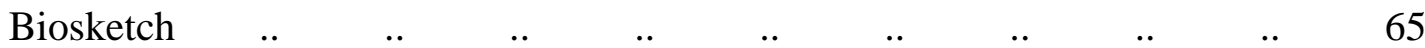

$\begin{array}{lllllllll}\text { Why EGTM for this study } & . . & . & . & . & . . & . . & . . & 66\end{array}$

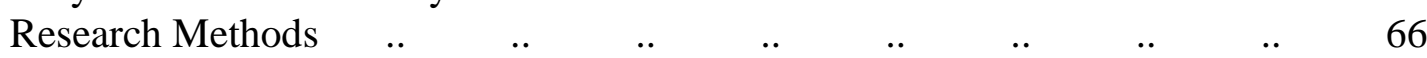

$\begin{array}{llllllllll}\text { Sample selection } & . . & . . & . . & . . & . . & . . & . . & . . & 66\end{array}$

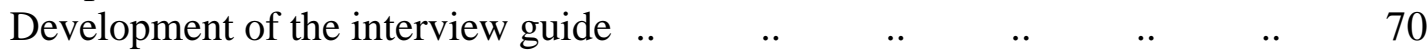

Data collection $\quad, \quad, \quad, \quad, \quad, \quad, \quad, \quad, \quad, \quad, \quad, \quad 72$

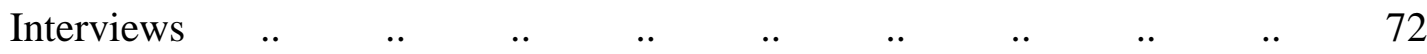

$\begin{array}{llllllllllll}\text { Coding } & . . & . . & . . & . . & . . & . & . & . & . . & . . & 73\end{array}$

$\begin{array}{lllllllllll}\text { Data analysis } & . . & . . & . . & . . & . . & . . & . . & . . & . . & 73\end{array}$

$\begin{array}{lllllllll}\text { Demonstration of rigour } & . . & . . & . . & . . & . . & . . & . . & 76\end{array}$

Considerations of transparency and trustworthiness through reflexivity and

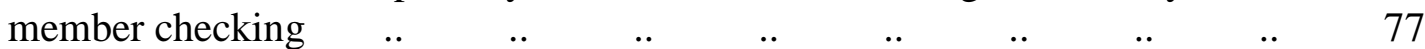

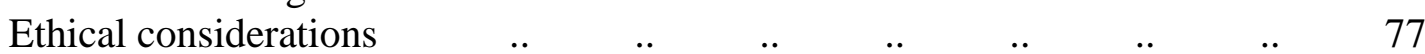

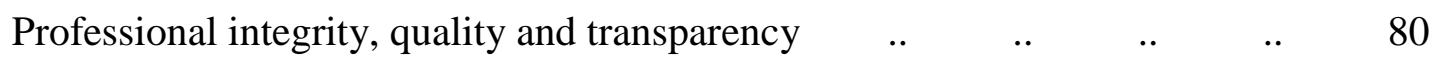

Relations with, and responsibilities to, sponsors or funders, independence of

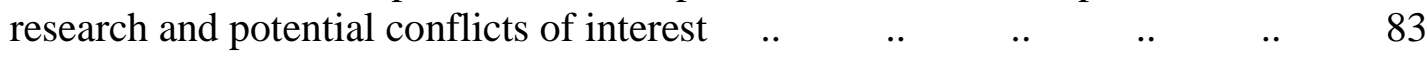

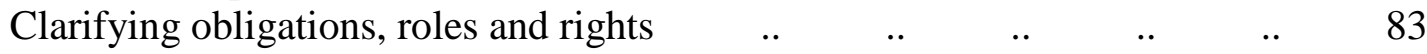

Pre-empting outcomes, negotiations about research and accurate reporting .. $\quad 84$

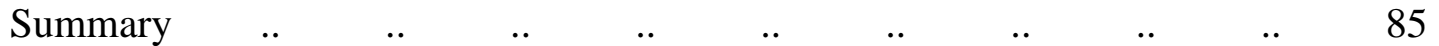

Chapter Four - Perceptions of emergency care staff of those who Self Harm: A systematic review of the quantitative literature

$\begin{array}{lllllllllll}\text { Introduction } & . . & . . & . . & . . & . . & . . & . . & . . & . . & 90\end{array}$

$\begin{array}{lllllllllll}\text { Methods } & . . & . . & . . & . . & . . & . . & . . & . . & . . & 92\end{array}$

$\begin{array}{llllllllll}\text { Search strategy } & . . & . . & . . & . . & . . & . . & . . & . . & 92\end{array}$

$\begin{array}{llllllllll}\text { Eligibility criteria } & . . & . . & . . & . . & . . & . . & . . & . . & 92\end{array}$

$\begin{array}{llllllllll}\text { Quality assessment .. } & . . & . & . & . . & . . & . . & . . & . . & 94\end{array}$

$\begin{array}{lllllllll}\text { Data extraction and synthesis } & . . & . . & . . & . . & . . & . . & 94\end{array}$

$\begin{array}{llllllllllll}\text { Results } & . & . & . & . . & . . & . . & . . & . . & . . & . . & 94\end{array}$

Critique of quality of the literature included in the systematic review $\quad . . \quad 98$

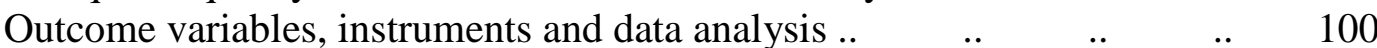

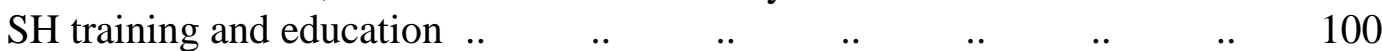

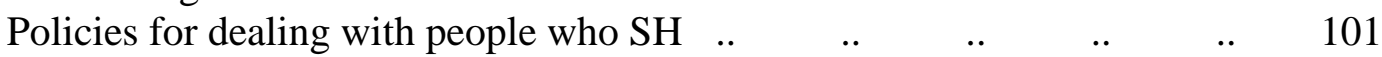

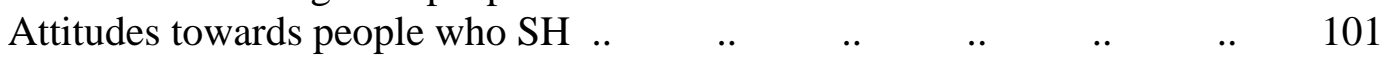

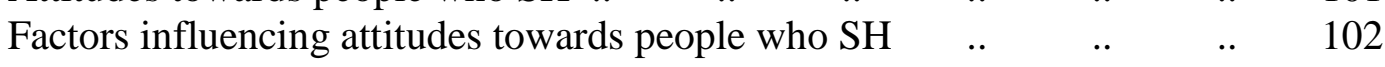

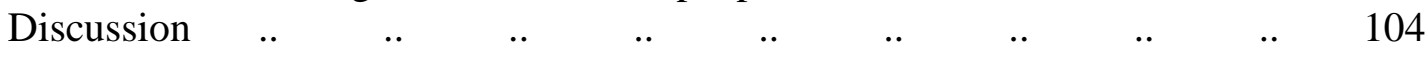

$\begin{array}{llllllllllll}\text { Limitations } & . . & . . & . . & . . & . & . & . & . . & . . & . . & 108\end{array}$

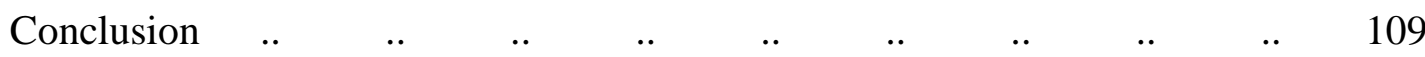

Chapter Five - Perceptions of paramedics and emergency staff about the care they provide to people who Self Harm: Constructivist metasynthesis of the qualitative literature

$\begin{array}{llllllllllll}\text { Introduction } & . . & . . & . . & . . & . . & . . & . . & . . & . . & 111\end{array}$

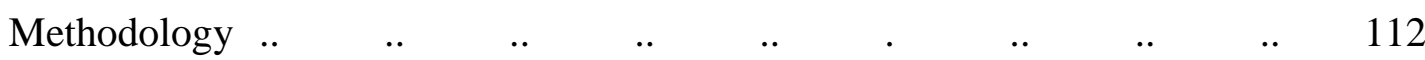

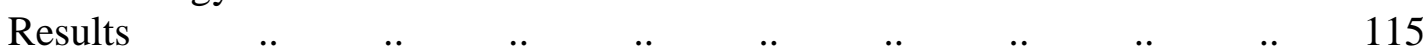

$\begin{array}{llll}\text { Critique of the quality of the literature included in metasynthesis .. } & \text {.. } & 115\end{array}$

$\begin{array}{llllllllllll}\text { Discussion } & . . & . . & . . & . . & . & . & . & . . & . . & . . & 130\end{array}$

$\begin{array}{llllllllllll}\text { Limitations } & . . & . . & . . & . . & . . & . . & . . & . . & . . & 133\end{array}$

$\begin{array}{llllllllllll}\text { Conclusion } & . . & . . & . . & . . & . . & . & . & . . & . . & . . & 134\end{array}$ 


\section{Chapter Six - Results}

Introduction

Categories:

Category 1: Context.

Category 2: Judgements and values

Category 3: Isolation and system failure

Category 4: Managing Risk

Category 5: Confidence and Competence at boundary of mental and $\begin{array}{llllllllll}\text { physical health needs .. } & . & . & . & . & \end{array}$

Category 6: Professional, legal and ethical tensions

\section{Chapter Seven - Final Evolved Grounded Theory}

Basic Social Process (BSP) in paramedic care for Self Harm: Decision making in a context of risk Final Grounded Theory:

Wicked Complexity of paramedic care for people who Self Harm: a

Grounded Theory

Usual factors

Heightened factors ..

Factors specific to Self Harm

Conclusion

\section{Chapter Eight - Discussion}

$\begin{array}{llllllllllll}\text { Introduction } & . . & . . & . . & . . & . . & . . & . . & . . & . . & 172\end{array}$

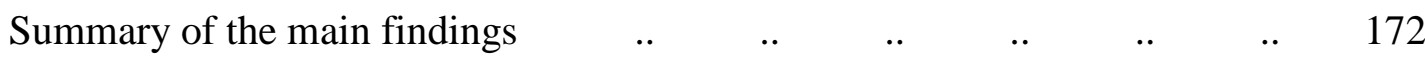

What has this study found and what does it add to our understanding? $\quad$.. 173

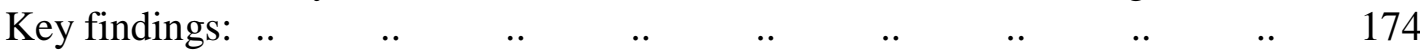

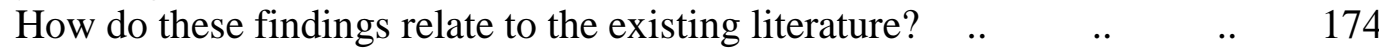

Influence of the context of paramedic care for people who Self Harm $\quad$.. 175

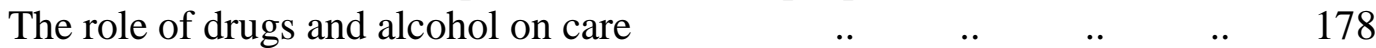

$\begin{array}{llll}\text { Competence with physical mental health care needs } & \text {.. } & \text {. } & 179\end{array}$

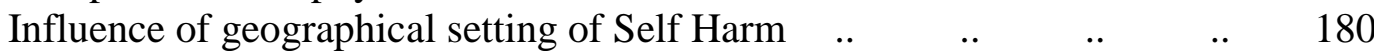

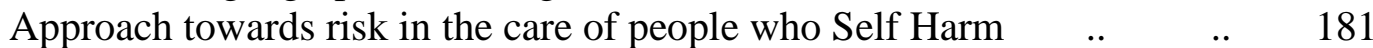

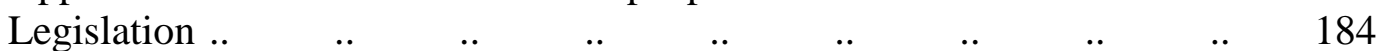

Social value and stigma in paramedic care for people who Self Harm .. 189

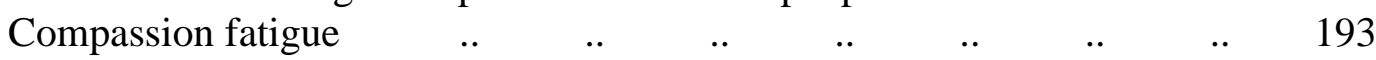

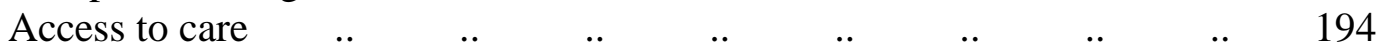

Training and education for paramedics in Self Harm care $\quad$.. $\quad$. $\quad 195$

Case management and joint police mental health working initiatives $\quad$.. 196

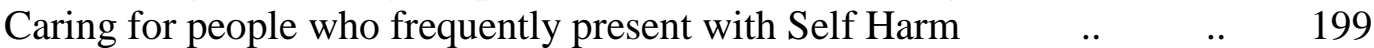

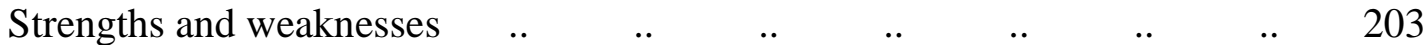

$\begin{array}{llllllllll}\text { Insider status of the researcher } & . . & . . & . . & . . & . . & . & & 206\end{array}$

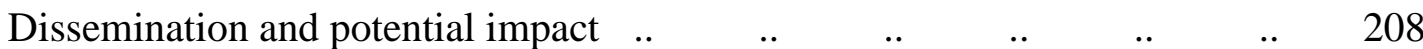

$\begin{array}{lllll}\text { Improvements in paramedic care for people who Self Harm .. } & \text {.. } & \text {. } & 212\end{array}$

$\begin{array}{lllllllll}\text { Implications for future research } & . . & . . & . . & . . & . . & . . & 214\end{array}$

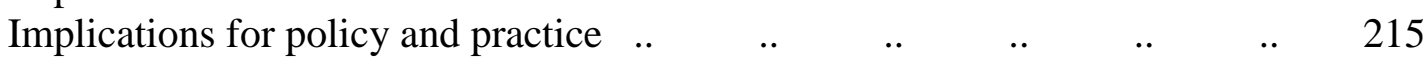




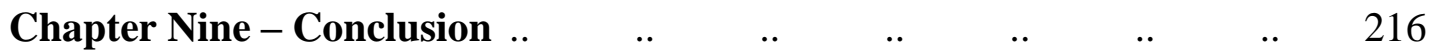

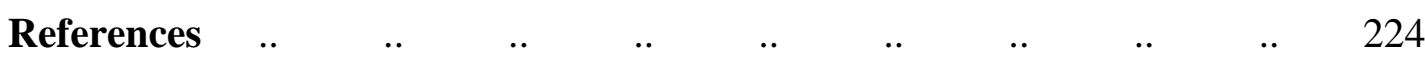

\section{Appendices}

Appendix A: NICE (2004 p.29) recommend a range of key aims and objectives in the treatment of Self Harm, , , , , .. 279

Appendix B. NICE (2004) key priorities for implementation in care for

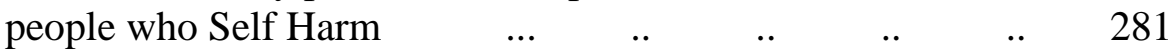

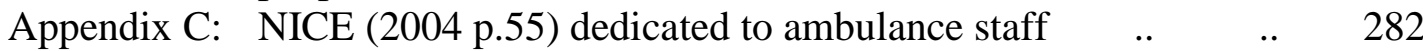

Appendix D: Royal College of Psychiatrists RCPsych (2006) guidelines in relation to ambulance staff care for people who Self Harm 284

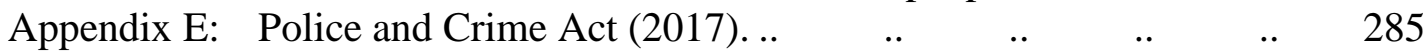

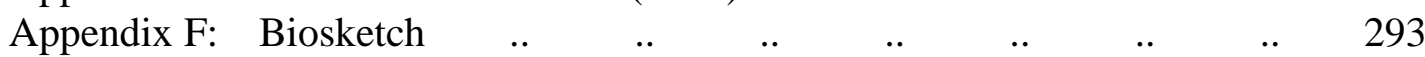

Appendix G Participant information sheet, Demographics Consent

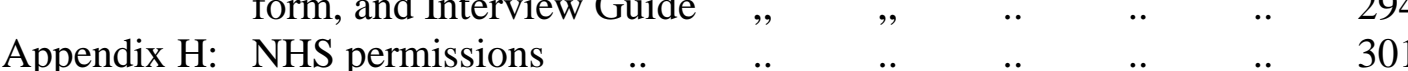

Appendix I: $\quad$ Rees, N. Rapport, F. Thomas, G. John, A. Snooks, H. (2014)

Perceptions of Paramedic and Emergency Care Workers of those who Self Harm: A Systematic Review of the Quantitative Literature. Journal of Psychosomatic Research. 77: 449-456 $\quad$.. $\quad$.. 303

Appendix J: Rees, N. Rapport, F. Snooks, H. (2015) Perceptions of paramedics and emergency staff about the care they provide to people who Self Harm: Constructivist Metasynthesis of the qualitative literature. Journal of Psychosomatic Research. 78: 529-535 , , . . 304

Appendix K: Truncated version of the metasynthesis coding book.. $\quad$.. 305

Appendix L: Rees N, Porter A, Rapport F, Hughes S, John A (2018)

Paramedics' perceptions of the care they provide to people who self-harm: A qualitative study using evolved grounded theory methodology. PLOS ONE 13(10): e0205813.

https://doi.org/10.1371/journal.pone.0205813

Appendix M: $\quad$ Truncated representation of the coding book for study proper and the interrelationship within codes $\quad . . \quad$..

Appendix N: Activities to include meetings, peer reviewed oral and poster presentations at conferences, publications, and $\begin{array}{llllllll}\text { supporting position statements } & . & . & & . . & & . . & 320\end{array}$

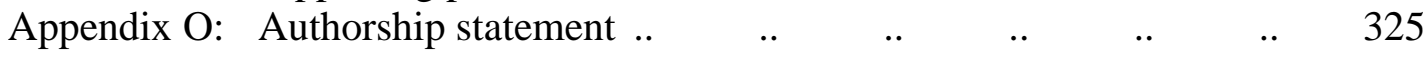




\section{Table of Figures}

Figure 1: Incidence of Self Harm per 100,000 in UK population by age and gender 1996-2005 (Hawton 2007 p.565)...

Figure 2: JRCALC (2006 p.191) Suicide and Self-harm Risk Assessment Form.. , , , , , , , , , ,

Figure 3: Age-standardised suicide rates by sex, deaths registered between

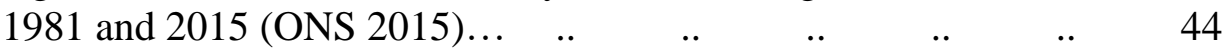

Figure 4: Strauss and Corbin (1998) induction, deduction and verification 73

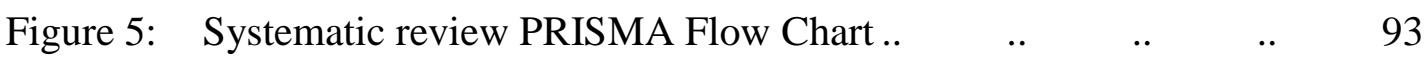

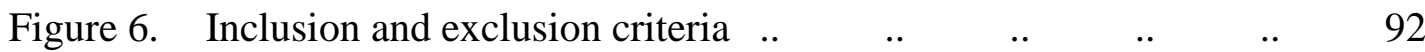

Figure 7 Systematic review data abstraction form , , , , , , , 95

Figure 8. Metasynthesis PRISMA Flow Chart , , , , , , , 118

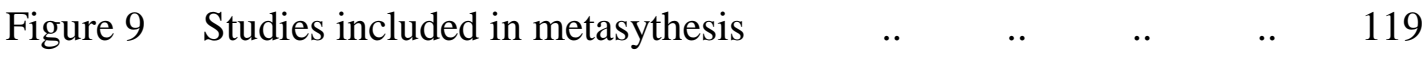

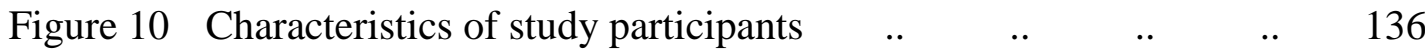

Figure 11. Overview of Grounded Theory (GT): Wicked complexity in

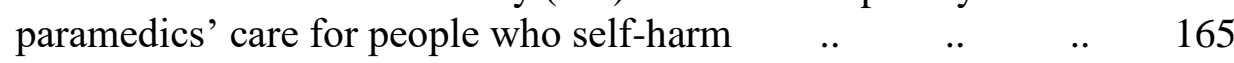




\section{Acknowledgements}

I would like to express my sincere gratitude to all those individuals who have made this study possible and who have supported me at every step. Firstly, I would like to thank the paramedics who participated in the study and who shared their rich and detailed accounts of this important topic area; the work would not have been possible without them. I am deeply indebted to my most respected supervisors throughout this study including Dr Alison Porter, Prof Ann John, Prof Frances Rapport and Prof Helen Snooks and I would like to thank them most sincerely for their invaluable support, guidance and assistance, and for being available and supervising me very patiently and calmly, even at their busiest moments. I would also like to thank Sarah Hughes for assistance with coding.

I would like to acknowledge the support and encouragement of my dearest friends and former colleagues Mr Richard Whitfield and Mrs Jane Turner, who encouraged me when stepping out of a clinical career as a paramedic to focus my energies on this intense period of study. I would also like to thank Dr Brendan Lloyd, my manager for the greater proportion of the study, who again provided me with the most patient support and encouragement. The Research \& Development (R\&D) Forum of the Welsh Ambulance Services NHS Trust is made up of a range of leaders, clinicians and academics with an interest in supporting ambulance services research to provide the highest quality evidence-based care. I would like to acknowledge their support for me and this study throughout many operational, leadership and organisational changes. Many challenging areas were revealed during this study due to the complex and sensitive nature of its focus, but the members of the R\&D Forum and leadership within WAST provided steadfast support and reassurance in allowing such sensitive research to be conducted.

The study was supported by a FALCK Foundation research grant, which enabled me to undertake fieldwork and to attend and present the work at many prestigious conferences. These opportunities enhanced my confidence and profile as an aspiring researcher paramedic. The College of Paramedics have followed this study closely over its lifetime and have drawn upon its findings to develop policy and position 
statements for Government consultations. I would therefore like to thank the College of Paramedics for this support.

The Journal of Psychosomatic Research, International Journal of Law \& Psychiatry and Plos One published academic papers arising from the study reported in this thesis. To the editors, peer reviewers and many others who enabled these peer reviewed articles to be published, thank you.

Over the last seven years I have been on a journey of discovery, both personally and academically, as I have undertaken the task of completing this thesis and the associated research. Because of this at times tumultuous personal and academic journey, I have grown both as a person and as an academic and it has brought both joy and sadness. I would like to thank my brothers, sisters and ever supportive mother for their unending support. To those friends and family who sadly passed away before being able to see this thesis completed, I also acknowledge their support. There are many other people, too numerous to mention by name, who have helped me in this journey but I sincerely thank them all for their support over the last eight years. Finally. I would like to thank my beloved wife Rebecca, my daughter Ffion and son Lloyd who have encouraged and supported me to complete this journey. 


\section{List of abbreviations}

\begin{tabular}{|c|c|}
\hline $\mathrm{A} \& \mathrm{E}$ & Accident and Emergency \\
\hline AMI & Acute Myocardial Infarction \\
\hline AMHP & Approved Mental Health Professional \\
\hline BPA & British Paramedic Association \\
\hline BSA & British Sociological Association \\
\hline BSP & Basic Social Process \\
\hline $\mathrm{BSc}$ & Bachelor of Science \\
\hline $\mathrm{CoP}$ & College of Paramedics \\
\hline $\mathrm{CCC}$ & Clinical Contact Centre \\
\hline $\mathrm{CHD}$ & Coronary Heart Disease \\
\hline $\mathrm{DOH}$ & Department of Health \\
\hline DipHE & Diploma of Higher Education \\
\hline ED & Emergency Department \\
\hline EGT & Evolved Grounded Theory \\
\hline EGTM & Evolved Grounded Theory Methodology \\
\hline EMS & Emergency Medical Services \\
\hline EOC & Emergency Operations Centre \\
\hline EMT & Emergency Medical Technician \\
\hline ESRC & Economic and Social Research Council \\
\hline GAfREC & Governance Arrangements for Research Ethics Committees \\
\hline GP & General Practitioner \\
\hline GT & Grounded Theory \\
\hline $\mathrm{HCP}$ & Health Care Provider \\
\hline $\mathrm{HCPC}$ & Health \& Care Professions Council \\
\hline HEMS & Helicopter Emergency Medical Service \\
\hline HEI & Higher Education Institution \\
\hline HPC & Health Professions Council \\
\hline IHCD & Institute for Health and Care Development \\
\hline JRCALC & Joint Royal Colleges Ambulance Liaison Committee \\
\hline LAS & London Ambulance Service \\
\hline LPFT & Lincolnshire Partnership Foundation Trust \\
\hline MI & Myocardial Infarction \\
\hline MCA & Mental Capacities Act \\
\hline MHA & Mental Health Act \\
\hline NCCMH & National Collaborating Centre for Mental Health \\
\hline NHS & National Health Service \\
\hline NHSTD & National Health Service Training Directorate \\
\hline NICE & The National Institute for Health and Care Excellence \\
\hline NIHR & National Institute for Health Research \\
\hline NISCHR & National Institute for Social Care and Health Research \\
\hline NRES & National Research Ethics Service \\
\hline NSF & National Service Framework \\
\hline NSF MH & National Service Framework on Mental Health \\
\hline NSSI & Non-Suicidal Self-Injury \\
\hline ONS & Office of National Statistics \\
\hline PRS & Proportionate Review Service \\
\hline $\mathrm{R} \& \mathrm{D}$ & Research and Development \\
\hline
\end{tabular}


RCPsych

REC

SAS

SDB

SDM

$\mathrm{SH}$

UK

WHO
Royal College of Psychiatrists

Research Ethics Committee

Scottish Ambulance Service

Social Desirability Bias

Shared Decision Making

Self Harm

United Kingdom

World Health Organisation 


\section{Definitions/terminology}

There are many terms used throughout this thesis. These are explained in the following text:

\begin{tabular}{|c|c|}
\hline Accident and Emergency & $\begin{array}{l}\text { Also known as Emergency Department (ED) } \\
\text { emergency room (ER), emergency ward (EW) or } \\
\text { casualty department, this is a medical treatment facility } \\
\text { specializing in emergency medicine and the acute care } \\
\text { of patients who present without prior appointment }\end{array}$ \\
\hline Acute Myocardial Infarction & $\begin{array}{l}\text { Sometimes called a heart attack. The term "myocardial } \\
\text { infarction" focuses on the heart muscle, which is called } \\
\text { the myocardium, and the changes that occur in it due } \\
\text { to the sudden deprivation of circulating blood }\end{array}$ \\
\hline Ambulance Trust & $\begin{array}{l}\text { There are ten regional ambulance trusts in the NHS in } \\
\text { England and Wales responsible for providing } \\
\text { Emergency and Urgent care and transportation }\end{array}$ \\
\hline $\begin{array}{l}\text { Approved Mental Health } \\
\text { Professional }\end{array}$ & $\begin{array}{l}\text { A person who is warranted or authorised to make } \\
\text { certain legal decisions and applications under } \\
\text { the Mental Health Act 1983. These can include } \\
\text { doctors, social workers, psychiatric nurses and } \\
\text { occupational therapists }\end{array}$ \\
\hline $\begin{array}{l}\text { British Paramedic } \\
\text { Association }\end{array}$ & $\begin{array}{l}\text { A professional association to represent the paramedic } \\
\text { profession and undertake the self-regulation of } \\
\text { paramedic standards and education required by the } \\
\text { HPC. In } 2009 \text { its name was changed to the College of } \\
\text { Paramedics. This reflected the association's ambitions } \\
\text { to be not only the professional voice of pre-hospital } \\
\text { ambulance clinicians, but also the driving authority for } \\
\text { increasing sections of pre-hospital clinical care } \\
\text { education, training, proficiency and continuous } \\
\text { professional development }\end{array}$ \\
\hline
\end{tabular}




\begin{tabular}{|c|c|}
\hline $\begin{array}{l}\text { British Sociological } \\
\text { Association }\end{array}$ & $\begin{array}{l}\text { A scholarly and professional society for sociologists in } \\
\text { the United Kingdom founded in 1951. It publishes the } \\
\text { academic journals Sociology, Work, Employment and } \\
\text { Society and Cultural Sociology (with SAGE } \\
\text { Publications) as well as its membership } \\
\text { newsletter Network and a monthly eNewsletter. }\end{array}$ \\
\hline College of Paramedics & $\begin{array}{l}\text { The recognised professional body for paramedics in } \\
\text { the United Kingdom. The role of the College is to } \\
\text { promote and develop the paramedic profession across } \\
\text { England, Scotland, Wales and Northern Ireland. }\end{array}$ \\
\hline Care $\mathrm{p}$ & $\begin{array}{l}\text { A planned, coordinated and multidisciplinary practice. } \\
\text { This can span across different health and social care } \\
\text { sectors, as well as the statutory, voluntary and private } \\
\text { sectors. }\end{array}$ \\
\hline $\begin{array}{l}\text { Clinical Contact Centre } \\
\text { (Wales) }\end{array}$ & $\begin{array}{l}\text { Coordinates and responds mobile health care } \\
\text { resources, providing telephone triage, advice, and } \\
\text { facilitating an online health care resource. The services } \\
\text { provided include access to nurse telephone triage, } \\
\text { health information or advice, online health } \\
\text { information, resources and advice, out of hours call } \\
\text { handling where commissioned, emergency ambulance } \\
\text { services, non-emergency transportation planning and } \\
\text { control and dental help line. Formerly known as } \\
\text { Ambulance Control, Ambulance Services in England } \\
\text { use the term Emergency Operations Centre }\end{array}$ \\
\hline $\begin{array}{l}\text { Department of Health and } \\
\text { Social Care }\end{array}$ & $\begin{array}{l}\text { Formally created in } 1988 \text { as the Department of Health } \\
\text { (DoH) and responsible for strategic leadership and } \\
\text { funding for both health and social care in England. } \\
\text { After the } 2018 \text { British cabinet reshuffle, the } \\
\text { department was renamed the Department of Health and } \\
\text { Social Care. It oversees the English National Health } \\
\text { Service (NHS). The Department is led by the Secretary } \\
\text { of State for Health and Social Care with two Ministers }\end{array}$ \\
\hline
\end{tabular}




\begin{tabular}{|c|c|}
\hline & $\begin{array}{l}\text { of State and three Parliamentary Under-Secretaries of } \\
\text { State. }\end{array}$ \\
\hline Emergency Department & $\begin{array}{l}\text { Also known as an accident \& emergency } \\
\text { department (A\&E), emergency room (ER), emergency } \\
\text { ward (EW) or casualty department, is a medical } \\
\text { treatment facility specializing in emergency medicine, } \\
\text { the acute care of patients who present without prior } \\
\text { appointment; either by their own means or by that of } \\
\text { an ambulance. The emergency department is usually } \\
\text { found in a hospital or other primary care centre. }\end{array}$ \\
\hline $\begin{array}{l}\text { Evolved Grounded Theory } \\
\text { Methodology (EGT) }\end{array}$ & $\begin{array}{l}\text { A term to describe the spiral of methodological } \\
\text { development and revision of the original methodology } \\
\text { of Grounded Theory introduced by Strauss and Corbin. } \\
\text { EGT follows a constructivist perspective and positions } \\
\text { the researcher as the author of a reconstruction of } \\
\text { experience and meaning. }\end{array}$ \\
\hline $\begin{array}{l}\text { Emergency Medical } \\
\text { Services }\end{array}$ & $\begin{array}{l}\text { This term refers to the treatment and transport of } \\
\text { people in crisis health situations that may be life } \\
\text { threatening. EMS are staffed by trained medical } \\
\text { professionals, called Emergency Medical Technicians, } \\
\text { trained in a limited scope of acute pre hospital care } \\
\text { practices, and paramedics who deliver advanced care, } \\
\text { and are increasingly becoming registered health } \\
\text { professionals internationally }\end{array}$ \\
\hline $\begin{array}{l}\text { Emergency Operations } \\
\text { Centre }\end{array}$ & $\begin{array}{l}\text { Also known as Ambulance Control or Clinical Contact } \\
\text { Centre. An emergency operations centre (EOC) is a } \\
\text { central command and control facility responsible for } \\
\text { EMS and Ambulance Services }\end{array}$ \\
\hline $\begin{array}{l}\text { Emergency Medical } \\
\text { Technician }\end{array}$ & $\begin{array}{l}\text { Clinicians trained to respond quickly to emergency } \\
\text { situations regarding medical issues, traumatic injuries } \\
\text { and accidents. Most commonly found working } \\
\text { in ambulances, but should not be confused with } \\
\text { "ambulance drivers" or "ambulance attendants" - }\end{array}$ \\
\hline
\end{tabular}




\begin{tabular}{|c|c|}
\hline & $\begin{array}{l}\text { ambulance staff who in the past were not trained in } \\
\text { emergency care or driving. EMTs operate under a } \\
\text { limited scope of practice. }\end{array}$ \\
\hline General Practitioner & $\begin{array}{l}\text { A medical doctor based in the community who treats } \\
\text { patients with minor or chronic illnesses and refers } \\
\text { those with serious conditions to a hospital. }\end{array}$ \\
\hline Grounded Theory & $\begin{array}{l}\text { Grounded theory is an inductive, theory discovery } \\
\text { methodology that allows the researcher to develop a } \\
\text { theoretical account of the general features of a topic } \\
\text { while simultaneously grounding the account in } \\
\text { empirical observations or data }\end{array}$ \\
\hline $\begin{array}{l}\text { Health and Care Professions } \\
\text { Council }\end{array}$ & $\begin{array}{l}\text { The regulatory body for allied health professions } \\
\text { including paramedics }\end{array}$ \\
\hline $\begin{array}{l}\text { Helicopter Emergency } \\
\text { Medical Service }\end{array}$ & $\begin{array}{l}\text { A helicopter or, less commonly, a fixed wing aircraft, } \\
\text { used to evacuate a person who requires } \\
\text { immediate medical attention that cannot be provided } \\
\text { on site }\end{array}$ \\
\hline $\begin{array}{l}\text { Joint Royal Colleges } \\
\text { Ambulance Liaison } \\
\text { Committee }\end{array}$ & $\begin{array}{l}\text { A committee which develops evidence based clinical } \\
\text { guidelines for UK ambulance services }\end{array}$ \\
\hline Mental Health Act (1983) & $\begin{array}{l}\text { An Act of the Parliament of the United Kingdom } \\
\text { which applies to people in England and Wales. It } \\
\text { covers the reception, care and treatment of mentally } \\
\text { disordered persons, the management of their property } \\
\text { and other related matters. }\end{array}$ \\
\hline $\begin{array}{l}\text { Mental Capacities Act } \\
\text { (2005) }\end{array}$ & $\begin{array}{l}\text { An Act of the Parliament of the United Kingdom } \\
\text { designed to protect and empower people who may lack } \\
\text { the mental capacity to make their own decisions about } \\
\text { their care and treatment. It applies to people aged } 16 \\
\text { and over. }\end{array}$ \\
\hline National Health Service & $\begin{array}{l}\text { The publicly funded national healthcare system for } \\
\text { England and one of the four National Health Services } \\
\text { for each constituent country of the United Kingdom. }\end{array}$ \\
\hline
\end{tabular}




\begin{tabular}{|c|c|}
\hline $\begin{array}{l}\text { National Health Service } \\
\text { Training Directorate }\end{array}$ & The NHS training body in the $1980 \mathrm{~s}-90 \mathrm{~s}$ \\
\hline $\begin{array}{l}\text { National Institute for Health } \\
\text { and Care Excellence }\end{array}$ & $\begin{array}{l}\text { Provides UK national guidance and advice to improve } \\
\text { health and social care }\end{array}$ \\
\hline $\begin{array}{l}\text { National Institute for Health } \\
\text { Research (NIHR) }\end{array}$ & $\begin{array}{l}\text { Funded through the Department of Health to improve } \\
\text { the health and wealth of the nation through research. It } \\
\text { provides support for applied health research focused } \\
\text { on the needs of patients and the public. Since its } \\
\text { establishment in April 2006, the NIHR has } \\
\text { transformed research in the NHS. }\end{array}$ \\
\hline $\begin{array}{l}\text { National Institute for Social } \\
\text { Care and Health Research } \\
\text { (Re-named Health and Care } \\
\text { Research Wales) }\end{array}$ & $\begin{array}{l}\text { The research and development arm of Welsh } \\
\text { Government in the early 2000s which set policy and } \\
\text { provided an infrastructure to support the governance } \\
\text { and delivery of research within NHS Wales }\end{array}$ \\
\hline $\begin{array}{l}\text { National Service } \\
\text { Framework }\end{array}$ & $\begin{array}{l}\text { A series of condition specific frameworks for quality } \\
\text { improvement and fair access introduced into the UK } \\
\text { NHS in the1980-1990s }\end{array}$ \\
\hline $\begin{array}{l}\text { Non-Suicidal Self-Injury } \\
\text { (NSSI) }\end{array}$ & $\begin{array}{l}\text { Direct, deliberate destruction of one's own body tissue } \\
\text { in the absence of intent to die. These features } \\
\text { distinguish it from behaviour whose harmful } \\
\text { consequences are unintended (e.g., lung cancer from } \\
\text { smoking), and from suicidal behaviour whose } \\
\text { prevalence, correlates, course, and response to } \\
\text { treatment differ. Culturally sanctioned bodily } \\
\text { modification, such as tattooing or body piercing, is not } \\
\text { classified as NSSI. (Nock } 2009 \text { p.3) }\end{array}$ \\
\hline Paramedic & $\begin{array}{l}\text { Provides specialist care and treatment to patients who } \\
\text { are either acutely ill or injured. They can administer an } \\
\text { advanced range of drugs and carry out certain surgical } \\
\text { techniques. Paramedics are increasingly becoming } \\
\text { registered health professionals across the world }\end{array}$ \\
\hline
\end{tabular}




\begin{tabular}{|l|l|}
\hline Parasuicide & $\begin{array}{l}\text { An act with non-fatal outcome, in which an individual } \\
\text { deliberately initiates a non-habitual behaviour that, } \\
\text { without intervention from others, will cause self-harm, } \\
\text { or deliberately ingests a substance in excess of the } \\
\text { prescribed or generally recognised therapeutic dosage } \\
\text { and which is aimed at realising changes which the } \\
\text { subject desired via the actual or expected physical } \\
\text { consequences (Kerkhof et al, 1994) }\end{array}$ \\
\hline Parity of Esteem & $\begin{array}{l}\text { A principle enshrined in law, which seeks to value } \\
\text { mental health equally with physical health. }\end{array}$ \\
\hline Poyal College of & $\begin{array}{l}\text { The professional body responsible for education and } \\
\text { training, and setting and raising standards in psychiatry }\end{array}$ \\
\hline Shared Decision Making & $\begin{array}{l}\text { Is when health professionals and patients work } \\
\text { together on care or treatment options, fully exploring } \\
\text { their risks and benefits and putting people at the centre } \\
\text { of decisions about their own treatment and care. }\end{array}$ \\
\hline Self Harm & $\begin{array}{l}\text { The intentional act of self-poisoning or self-injury } \\
\text { irrespective of the type of motivation or degree of } \\
\text { suicidal intent. Thus it includes suicide attempts as } \\
\text { well as acts where little or no suicidal intent is } \\
\text { involved (e.g. where people harm themselves to reduce } \\
\text { internal tension, distract themselves from intolerable } \\
\text { situations, as a form of interpersonal communication }\end{array}$ \\
\hline
\end{tabular}




\section{Chapter One - Introduction}

Paramedics are registered health professionals specialising in emergency and prehospital care, but whilst paramedic practice has traditionally been developed for those presenting with life threatening emergencies such as serious trauma and acute medical emergencies (Lendrum et al 2000), the role of the paramedic is changing and increasingly involves assessing and managing people with non-life-threatening emergencies and mental health problems. People presenting with mental health problems can account for up to $10.7 \%$ of 999 calls to ambulance services, and of these 53\% relate to self-harm (SH) (Shaban 2005a, INVENT 2013), representing a significant patient group, which will require appropriate care and management. Despite this, patients who SH report low levels of satisfaction with the care they receive from clinical staff in general (Warm, Murray, \& Fox 2002), with some citing negative and hostile reactions from ambulance staff (Mental Health Foundation 2006). Whilst this situation may be disappointing to clinicians, ambulance services and patients, such substandard care may have significant implications for the patient, as the Royal College of Psychiatrists (RCPsych 2010) advises that the care patients receive at their first contact with a health professional may determine the quality and continuity of care they receive from then on, encourage further willingness to seek help when needed, improve quality of life and contribute to overcoming the Selfharming behaviour.

Paramedics report being ill-prepared for dealing with such patients presenting with mental health problems and that there are limited services available for those at risk following SH (Shaban 2005a). SH may therefore be a challenging presentation for paramedics, yet their views on how they respond to patients presenting with $\mathrm{SH}$ have not been comprehensively examined. This thesis uses Evolved Grounded Theory Methodology (EGTM) to explore this area of patient care from the point of view of paramedics working in an emergency ambulance service.

\section{Research aims and objectives}

Aim: To explore paramedics' perceptions of caring for those who $\mathrm{SH}$ in order to inform education policy and practice. 
Objective 1. To reveal paramedics' perceptions of caring for those who SH

Objective 2: To develop a theory on paramedics' perceptions of caring for $\mathrm{SH}$

\section{Background}

\section{Definition of Self Harm}

SH has been defined by the Royal College of Psychiatrists as an:
"intentional act of self-poisoning or self-injury irrespective of the type of motivation or degree of suicidal intent. Thus it includes suicide attempts as well as acts where little or no suicidal intent is involved (e.g. where people harm themselves to reduce internal tension, distract themselves from intolerable situations, as a form of interpersonal communication of distress or other difficult feelings, or to punish themselves." (RCPsych 2010)

The RCPsych (2010) definition has been adopted for this study as it is a standard definition used in UK SH policy and guidelines, such as those produced by NICE (2004). The RCPsych (2010) definition also covers the wide range of clinical presentations of SH paramedics may encounter, along with the breadth of severity of $\mathrm{SH}$, as it refers to those on the suicidal continuum ranging from high suicidal intent, through to acts that are aimed at coping with overwhelming feelings or may be more habitual. Defining SH is however not straightforward as there are various types of $\mathrm{SH}$, different motives and meanings for individuals, and different contexts in which SH occurs. These issues will be of importance to paramedics as they can encounter a whole range of different types of $\mathrm{SH}$, and motives for $\mathrm{SH}$ and experience the various contexts within which this occurs. There are a variety of terms used to describe behaviours that involve an individual inflicting harm on themselves. These include self-harm, self-injurious behaviour, self-mutilation, parasuicide, self-abuse and nonsuicidal self-injury (NSSI). One area of considerable debate around the definition of $\mathrm{SH}$ is the presence of suicidal intent. A definitive discussion of this debate is beyond the scope of this thesis; however, it is important to recognise the background to areas of challenge and relationship with suicidal intent, as these will impact on decision making and perceptions of paramedic care for people who SH. It is important to note that when paramedics are responding to people who SH they may not necessarily be able to identify if there is suicidal intent or not.

Non-suicidal self-injury (NSSI) can be thought of as a sub-group of $\mathrm{SH}$, and has been defined as: 
"direct, deliberate destruction of one's own body tissue in the absence of intent to die. These features distinguish it from behavior whose harmful consequences are unintended (e.g., lung cancer from smoking), and from suicidal behavior whose prevalence, correlates, course, and response to treatment differ. Culturally sanctioned bodily modification, such as tattooing or body piercing, is not classified as NSSI”. (Nock 2009 p.3)

Despite the prevalence of NSSI, little is known about why people engage in this behaviour. Nock (2007) presented a theoretical model of the development and maintenance of NSSI, and rather than a symptom of mental disorder, suggested that NSSI can be conceptualized as a harmful behaviour that can serve several intrapersonal (e.g. affect regulation) and interpersonal (e.g. help-seeking) functions. The definition by Nock (2007) differs from the RCPsych (2010) definition, which includes individuals in whom there may be suicidal intent. Nock (2007) also fails to reflect the details of motivations and behaviours involved with $\mathrm{SH}$ that are acknowledged in RCPsych (2010); namely behaviours aimed at reducing internal tension, distracting themselves from intolerable situations, or as a form of interpersonal communication of distress or other difficult feelings, or to punish themselves. The RCPsych (2010) definition therefore reflects the communicative and help seeking behaviours of $\mathrm{SH}$, which is important for this study as paramedics may be some of the early participants in this communication and are often the first health professionals in contact following $\mathrm{SH}$.

In 1986, the World Health Organisation (WHO) adopted the term parasuicide which is defined as:

"An act with non-fatal outcome, in which an individual deliberately initiates a non-habitual behaviour that, without intervention from others, will cause self-harm, or deliberately ingests a substance in excess of the prescribed or generally recognised therapeutic dosage and which is aimed at realising changes which the subject desired via the actual or expected physical consequences" (Kerkhof et al, 1994)

This definition differs in many ways to the RCPsych (2010) definition and, as with Nock (2007), whilst it does not exclude the motivation of suicide, it is not specifically mentioned. Linguistic challenges also exist with the prefix para, and its failure to fully clarify or show the magnitude or nature of the act of SH (De Leo et al 2004), and parasuicide has been replaced with terms such as suicide attempt and non- 
fatal suicidal behaviour (Gandhi et al 2016). One can also appreciate how such classification presents challenges, as clinically qualifying the intent to die is not always feasible.

Gandhi et al (2016) reviewed definitions of various forms of self-directed violent behaviours in India, comparing the usage of these definitions with the usage by World Health Organisation. They suggested that there is an international lack of consensus over the use of various forms of self-directed violent behaviours, and that most terms and definitions used are culturally informed, contextually relevant, evolve over time and, therefore, make the presentation of concrete guidelines difficult. The RCPsych (2010) however is the most robust definition for the purposes of this study, as it is one which is commonly used in the UK and culturally informed, it is the definition used in the NICE (2004) guidelines on SH care, and therefore appropriate within the context of this study. The RCPsych (2010) reflects the context in which the study took place, and refers to those who $\mathrm{SH}$ on the suicidal continuum ranging from high suicidal intent, through to acts that are aimed at coping with overwhelming feelings or more habitual behaviours.

\section{Forms that Self-Harm can take:}

Methods of SH can include burning, hanging, strangulation, scratching, banging or hitting body parts, and mutilation of parts of the body or interfering with wound healing. It may also involve other behaviours such as disembowelment, eye enucleation, degloving, amputation, castration, ingesting substances, inserting or removing objects through orifices or wounds, serious tampering with medical interventions, jumping from high places, asphyxiation, head banging, bone breaking, striking of self, and lack of self-care that results in a medical crisis (Fagan et al. 2010, Lanes 2009).

A person's choice of a SH is not necessarily static and people often switch from cutting to self-poisoning, for example (Owens et al 2015). The most common form of SH is cutting, and with a variety of implements and degrees of severity (RCPsych 2010). Cutting may also be more habitual and repetitively carried out over multiple occasions than other forms of SH (Lilley et al 2008, Evren et al 2010, Lanes 2009). 
However, Doshi et al (2005) found that in terms of presentation to ED, the most common method of SH was poisoning (68\%), followed by cutting or piercing (20\%). Unlike cutting, poisoning may be more influenced by the environment in which the patient is, as substances used to self-poison reflect trends in prescribing. In the UK, non-opiate analgesics such as paracetamol are the most widely used for $\mathrm{SH}$, whereas in developing countries, organophosphates, other pesticides and naturally occurring poisons are often used, especially in rural areas (Hawton et al 2007). Self-poisoning also has other added dangers linked to the context of the $\mathrm{SH}$; for instance, heavy drinking often precedes overdose, which increases the potential for further harm due to unpredictable interactions with alcohol and poisoning agents used in $\mathrm{SH}$, (Hawton et al 2007) or indeed any prescription drugs the individual may also be taking resulting in increased harm to the person.

The method of SH may well influence paramedics' perceptions of care for people who $\mathrm{SH}$, but irrespective of this, all methods of $\mathrm{SH}$ are associated with future risk of suicide, including self-cutting, which incurs no less of a risk than other methods (Bergen et al 2012, Cooper et al 2005, Hawton et al 2012). Patients who SH therefore need an emergency response from Ambulance Services and appropriate and urgent assessment and attention in hospital.

\section{Epidemiology of Self Harm}

SH is one of the five top causes of acute hospital admissions (RCPsych 2010), accounting for approximately 200,000 hospital attendances annually in England and Wales (Chan et al 2016). Hospital admissions due to SH rose from 91,341 in September 2005-August 2006 to 112,096 in September 2014-August 2015 (HSCIC 2015). These figures relate to demand for hospital services in England and Wales, with a population of approximately 60 million. From an ambulance service perspective, Duncan et al (2017) found that during 2011, there were 9,014 calls attended by the Scottish Ambulance Service (SAS), which serves a population of approximately 6 million; up to half of patients attended by SAS made at least one repeat call. Paramedics are therefore likely to encounter significant numbers of people who SH, many of whom present repeatedly, and it is therefore important to understand this care interaction. 
Despite the significant number of people who SH presenting to ambulance services, most people who SH hide the fact, with reports of up to $84 \%$ hiding it from their family, and only $12.5 \%$ reporting their first act of SH to others (SANE 2017). Indeed, findings from population-based studies indicate that only 10-20\% of those who engage in SH present to hospital (Pages et al. 2004, Ystgaard et al. 2003, Doyle et al 2015). This has led to suggestions that there is a hidden population of distressed individuals, including some that may have serious mental health problems (Ystgaard, et al 2008). The true scale is estimated to be 1 in 130 people, as many make efforts to avoid A\&E because of the unsympathetic response they expect there (MIND 2004). This hidden population poses potential problems for patients, carers and providers of services, resulting in few opportunities to intervene and support patients following $\mathrm{SH}$. Given the significant risk of suicide and health damaging effects of $\mathrm{SH}$, it is important to understand any barriers and opportunities in care, especially in those in early contact with people who SH (RCPsych 2010) such as paramedics.

People who SH tend to experience multiple difficulties, both psychological and social in nature (Haw \& Hawton 2008, Hume \& Platt 2007). Psychiatric illnesses, including depression, bipolar disorder, alcohol abuse, anxiety disorders, eating disorders, schizophrenia and substance abuse are common in people who SH, and McManus et al (2009) found the rate of a diagnosed mental illness of those who have died by suicide to be more than $80 \%$. In another study involving 1108 patients presenting for $\mathrm{SH}$ at general hospitals, alcohol or drug misuse was identified in $32 \%$, a severe mental illness in $7 \%$, depression in 29\%; anxiety/stress-related disorders in $13 \%$, and a further $4 \%$ were diagnosed with personality disorders (Dickson et al, 2009). Caring for people who SH therefore involves the need for understanding and assessment for a complex range of mental health and psychosocial problems, and this is made more challenging, because while SH may not always indicate a severe mental health condition, the person may be experiencing a period of 'transient distress' (Hawton et al 2006 a).

People of all ages and from all social and cultural backgrounds may $\mathrm{SH}$, however some groups are especially vulnerable (RCPsych 2010). SH occurs across the 
lifespan, but young people have disproportionately higher rates of SH (Fliege et al 2009). SH often starts in early adolescence, then increases between ages 16 to 25 , but is rare in those under 12 (Skegg 2005). Adolescent females are the group with the highest incidence of SH; (Figure 1) demonstrates the high rates of $\mathrm{SH}$ in adolescents.

\section{Incidence of deliberate self-harm}

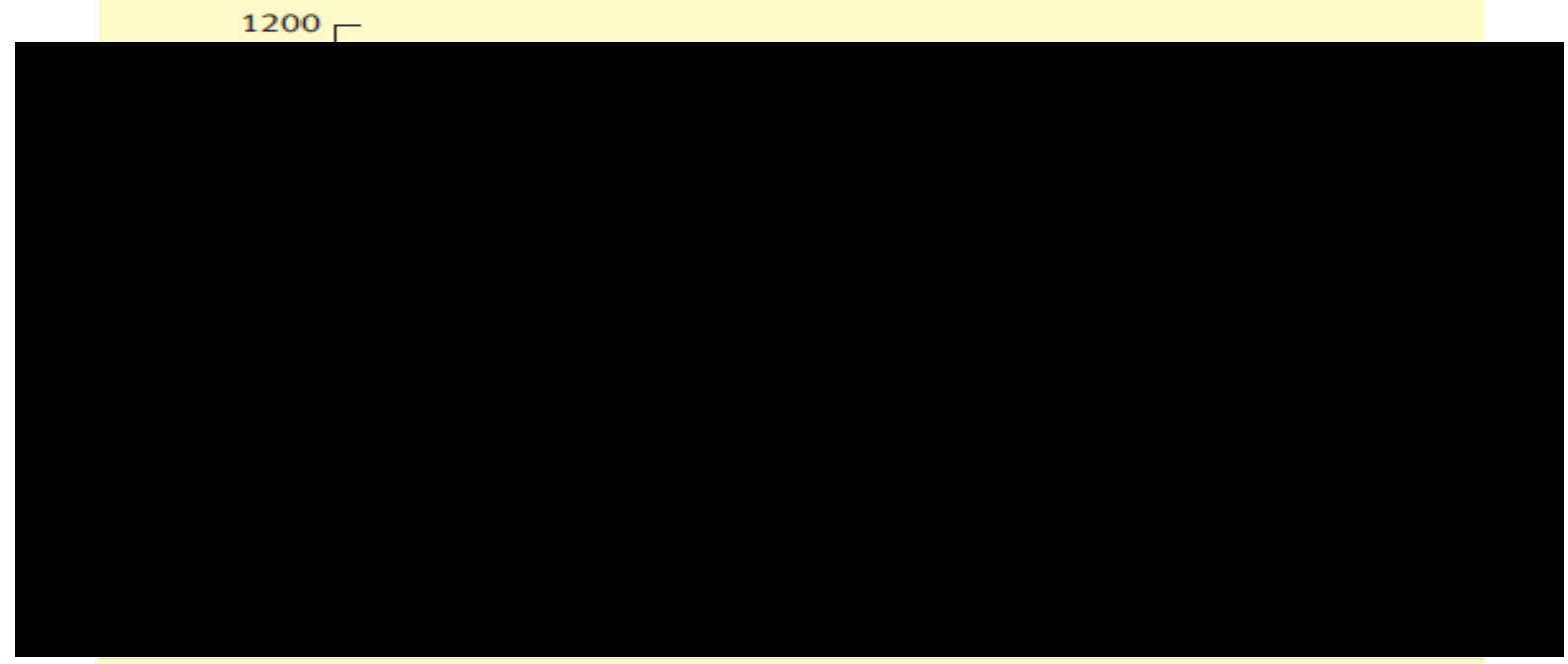

Data from Oxford, 1996-2005.

Fig 1. Incidence of SH per 100,000 in UK population by age and gender 19962005 (Hawton 2007 p.565)

Problems with schoolwork, friends, girlfriends/boyfriends, alcohol and drug use, and bullying are some of the psychosocial factors shown to have an impact on the risk of $\mathrm{SH}$ in this age range (de Kloet et al 2011). There is a higher than average incidence of SH among prisoners, armed forces veterans, asylum seekers, those bereaved by suicide, and some cultural minority groups (RCPsych 2010). It is highest in lower socioeconomic status individuals, and in urban areas characterized by socioeconomic deprivation, overcrowding and poor social integration (Hawton et al 2003). Single and divorced individuals are more at risk than those in other marital categories, and there is a strong link with alcohol abuse (Haw et al 2005). Unemployment markedly increases the risk of self-poisoning, being particularly high in those who are longterm unemployed. Platt et al (2005), however, argue that this is mediated by the effects of unemployment on mental health, by poverty and deprivation, and often this 
association reflects the fact that individuals with poor mental health, and at greater risk of SH, are more likely to become unemployed.

Whilst many people who SH have psychiatric disorders (Haw et al 2001) such as anxiety, and depression (Fliege et al 2009), Lanes (2009) recognises that factors in life histories of individuals increase the likelihood of engaging in $\mathrm{SH}$ behaviour and suicide attempts. These include: abuse and neglect during childhood, brain injury, lack of formal education, and mental illness (mood disorder or personality disorder). Sexual abuse and family dysfunction have also been found to be prevalent amongst those who SH (Klonsky et al 2007, Skegg 2005, Fliege et al 2009).

\section{Relationship between Self Harm and suicide}

In the UK, suicide is the third largest contributor to premature mortality (after heart disease and cancer) (World Health Organization, 2000). In 2014 there were 6,581 recorded cases of suicide in the UK and Republic of Ireland (Samaritans 2016). It is important however to note that these figures may not represent the true scale. Suicide statistics in England and Wales are derived from death certificates issued after coroners' inquests into unnatural or unexpected deaths. It has long been recognised that death certificate data are imperfect (Adelstein 1975), and most deaths given open verdicts are likely to be suicides (Linsley 2001). Narrative verdicts can be used by a coroner or jury instead of a short form verdict to express their conclusions as to the cause of death following an inquest (ONS 2011).

$\mathrm{SH}$ is a complex phenomenon and has been suggested to lie on a continuum of suicide that ranges from ideas, to SH, and finally suicide completions (Marris 2002), and along this continuum lie many who underestimate how lethal their behaviour could be (Stanley et al 2001). Likely consequences of their actions are that 1 person in every 100 appearing in hospital following SH dies by suicide within a year, and five per cent in the following decade (Hawton \& Fag 1998). SH elevates risk of suicide 50 to 100-fold within the year following SH. (Chan et al, 2016). Those who SH are also 100 times more likely than the general population to die by suicide (NICE 2004), presenting a clear link between SH and suicide, and any discussion around paramedic care for those who SH must recognise such a link. 


\section{Motivation and intent of Self Harm}

The literature suggests that while in some people the intention is to die, for others, $\mathrm{SH}$ is a behaviour without the intention or desire to die lying behind it. Rather, $\mathrm{SH}$ serves as a form of self-preservation, a mobilisation of help, and can be habitual or even addictive (Csipke \& Horne, 2008). It can be conducted as temporary escape from stress (Van Heeringen 2002), to punish oneself or get relief from a terrible state of mind (Scoliers et al 2008). In some, $\mathrm{SH}$ serves as a means to release anger, communicate distress to others, or resolve conflict, tension, or emotional pain (Walsh et al 2007, Lloyd-Richardson et al 2007, Fagan et al., 2010, Jeglic et al., 2005). Interviews with people who SH suggest motives for this behaviour may also include being able to manage extreme pain or unbearable situations (Mental Health Foundation, 2006; O'Connor et al, 2009). SH is therefore often a coping mechanism or as a distraction that brings relief, accompanied by a complex set of feelings including self-disgust and shame (Chapman et al 2006).

The motivating factors in SH have been expressed through research exploring experiences of patients. In a report by the Mental Health Foundation, for example, a patient told how:

'Cutting for me releases all the built up anger and frustration and pain I feel inside. (MHF 2006 p.18)

Whilst in a study by Harris (2012) a participant said:

"The purpose of some acts of self-harm is to preserve life... professionals sometimes find this a difficult concept to understand."

\section{Experience of the care interaction between health professionals and people who Self Harm}

Very little literature exists about paramedic care for people who SH. It is reported in the wider emergency care literature that many patients allege that the quality of care and attitudes from staff are unsatisfactory (NICE 2004, MIND 2004, Johnstone 1997, Warm, Murray, \& Fox 2002), with patients reporting negative and hostile reactions from ambulance and A\&E staff (Mental Health Foundation 2006). In a survey of those who had undertaken $\mathrm{SH}, 43 \%$ said that they had avoided emergency services because of previous negative experiences (NCCMH 2004). Some respondents to the survey recalled being told that they were wasting time and resources, which 
compounded their distress and led them to discharge themselves prematurely (NCCMH 2004). In a study by Royal College of Psychiatrists one SH respondent said:

'It was an awful experience, I would rather die than go back there' (A\&E) (RCPsych 2008 p.70),

This quote reflects the negative experience a patient had when attending the ED following $\mathrm{SH}$. The patient reflects on this awful experience, and how they would avoid such care from health services following SH. They also tell of the risks (of death) they were prepared to take in future to avoid going back to ED. Another respondent to a Mental Health Foundation study (Mental Health Foundation 2006) revealed similar dissatisfaction with their experience of attending ED following $\mathrm{SH}$, however they communicate the sense of a lack of care an interest in them from health staff, stating that:

"On the occasions I have been admitted to an $A \& E$ department they have concentrated on medically patching me up and getting me out. Never have I been asked any questions regarding whether this is the first time I have selfharmed or if I was to do it again or how I intend to deal with it." (Mental Health Foundation 2006 p31).

Patients who SH report a wide range of such negative experiences of care, feeling misunderstood and unsatisfied with health carers behaviour towards them (McHale \& Felton, 2010), and Cook et al (2004) argue that many SH patients find themselves ignored by health professionals, not just because of negative attitudes towards them, but also because they are perceived as difficult to deal with. In the hospital setting, staff reportedly distance themselves from such patients, characterising them as manipulative, 'attention seeking' or beyond help (Vivekananda 2000), yet often such perceptions are grounded in inaccuracies and myths (Anderson 2005). Whilst SH is a behaviour, it was recognised earlier that many who SH experience psychiatric illnesses and social problems (Haw \& Hawton 2008, Hume \& Platt 2007). Indeed, one study found that $80 \%$ of those who have died by suicide have a diagnosed mental illness (McManus et al 2009), therefore people who SH and present to paramedics may be suffering from a treatable mental health condition which requires appropriate assessment, management and after care. Above all, people who engage in $\mathrm{SH}$ want to be seen by empathetic health professionals who are able to listen, to be supportive and not judgemental (Burke et al 2008, RCPsych 2010, NICE 2013). 
It has been acknowledged that long-standing lack of parity between mental and physical health problems exists, and this has been recognised to be inequitable and socially unjust (RCPsych 2013). 'Parity of esteem' is a principle that has evolved which seeks to redress some of this disparity, and can be defined as: 'Valuing mental health equally with physical health' (RCPsych 2013). Parity of esteem was called for in the RCPsych report, Whole-Person Care: From Rhetoric to Reality (RCPsych 2013). This report highlighted the significant inequalities that exist between physical and mental health care, including preventable premature deaths, lower treatment rates for mental health conditions and an underfunding of mental healthcare relative to the scale and impact of mental health problems.

In keeping with the principle of parity of esteem, RCPsych (2010) hold that all SH patients deserve the same standard of care as those with, for example, myocardial infarction or acute asthma, arguing that "All patients who self-harm should have a full bio psychosocial assessment carried out by a skilled and experienced clinician" (RCPsych 2010 b. p141). This is also reflected in a raft of clinical guidelines (Royal College of Psychiatrists, 2004; National Collaborating Centre for Mental Health, 2004 NICE 2004), which agree that psychosocial assessment of personal circumstances, social context, mental state, risk, and needs are central to the clinical management of SH.

Kapur (2003) found that psycho social assessments can halve the risk of repetition of $\mathrm{SH}$. Despite this there is marked variability in the application of such psycho social assessments, with over 80,000 who attend $A \& E$ annually never receiving this assessment (NICE 2004). Such psychosocial assessments are conducted by mental health staff, and involve a comprehensive evaluation of factors such as an individual's personal situation, history of SH, family history and mental state (Kapur et al 2008, Hawton et al 2012). In a sample of 10,498 episodes of SH Kapur (2003) found that fewer than half of $\mathrm{SH}$ patients attending A\&E received assessment.

Paramedics are often the first professional encountered by those who threaten to harm themselves, engage in SH or die by suicide, yet there is limited published 
investigation of this care. Calls have been previously been made for research, and qualitative research in particular (Anderson 2005, NICE 2004), to focus upon such occupational groups who care for those who SH (NICE 2004, RCPsych 2010, Warm, Murray, \& Fox 2002),

\section{Role of police and detention in the care of people who $\mathrm{SH}$}

Police and paramedics often work together in the care of people who $\mathrm{SH}$, and have long been key front-line responders in mental health emergencies (Borum 2000). It has been estimated that incidents linked to mental health account for between 20 to $40 \%$ of police time, and such encounters rose by 33\% between 2011 and 2014 (Guardian 2016, Brown 2013). The link between SH and mental health conditions has previously been discussed, where depression, anxiety and alcohol misuse disorders are well-known risk factors for SH (Haw et al 2001), and people who SH experience multiple difficulties, both psychological and social in nature (Haw \& Hawton 2008, Hume \& Platt 2007). This poses significant clinical and legal challenges for police and paramedics caring for people who SH. During a mental health crisis, police and paramedics may have to manage the medical and physical consequences of SH, and also discern the impact of any psychosocial and mental health problem which may have influenced the SH.

A mental health crisis occurs in situations when an individual is faced with an experience that exceeds their ability to cope (Caplan 1964). The result can be functional decompensation and disturbances of behaviour, which may place the person in crisis or others at risk of harm (Penterman \& Nijman 2011). Police officers are often the first responders to individuals in crisis; they make the critical decision of who gets arrested, who gets medical services and who gets released, operating as gatekeepers into the mental health system (Canada, Angell, \& Watson 2010). Police officers have consequently been characterised as de facto psychiatric triage specialists, 'street-corner' psychiatrists or lay social workers (Durbin et al 2010, Lamb et al 2002, Matheson et al 2005, Compton et al 2009, Cumming et al 1965, Bitter et al 1967, Sheridan et al 1982, Telpim et al 1986, 1992, Borum 1998).

When police are confronted with a person with apparent mental health problems, they generally have three options: (a) transport them to a receiving psychiatric 
facility, (b) use informal verbal skills to de-escalate the situation, or (c) arrest or detain the person (Teplin 2000). In the UK, when faced with a patient who has selfharmed, police and paramedics may be required to detain the person for the safety of themselves or others, which may include the need for urgent medical attention or assessment of an ongoing mental health problem. These scenarios involve application of legislation such as the Mental Capacities Act (MCA 2005) and Mental Health Act (MHA 1983), which is discussed in more detail later in this thesis. However, one piece of mental health legislation which is sometimes used is Section 136 of the MHA (1983), which can be applied if:

"a constable finds in a place to which the public have access a person who appears to him to be suffering from mental disorder and to be in immediate need of care or control, the constable may, if he thinks it necessary to do so in the interests of that person or for the protection of other persons, remove that person to a place of safety"

Paramedics and police are on occasions faced with desperate situations requiring detaining of the person under Section 136 of the MHA 1983. Detaining a patient and denying them autonomy to prevent suicide following SH may be a very distressing practice (Otahbachi et al 2010), and there are rare occasions in which people are injured and even killed during this process (Huriash, 2001; Vickers, 2000). People who $\mathrm{SH}$ and are detained in this way therefore constitute a vulnerable group who may be at increased risk of injury and death (IPPC 2008). In recent years, a new technology has entered the detainment process, Tazers, which are hand held conducted electrical weapons (Dyer 2015) and in the rare instances where the police actually discharged Tazers, $67 \%$ of subjects were identified as mentally ill (The Justice Gap 2015).

Paramedics and police manage most encounters with people who SH with great skill and empathy, which involves a balance of protecting the patient and others from harm, but as indicated, there is potential for further harm and distress. The interaction between police and paramedics in caring for people who $\mathrm{SH}$ involves a complex range of clinical, ethical and legislative issues aimed at providing the highest standards of care, protecting the patient and others. 


\section{Overview of thesis}

The following chapters of this thesis will present the evolution of a Grounded Theory exploring paramedics' perceptions of caring for those who SH. This work begins with a brief review exploring the context of paramedic care for people who $\mathrm{SH}$, presented in Chapter Two. Chapter Three presents the study methodology and introduces Evolved Grounded Theory Methodology (EGTM). A rigorous systematic review of the quantitative literature and metasynthesis of the qualitative literature were conducted and published in peer reviewed journals (Rees et al 2014, 2015); these are presented as chapters within Chapters Four and Five. Chapter Six presents findings, which again have been published in a peer reviewed journal (Rees et al 2018), and includes the six emergent categories describing paramedics' perceptions of caring for people who SH, which are: Context; Judgements and Values; Isolation and System Failure; Managing Risk; Competence at the boundary of mental and physical health needs; Professional, Legal and Ethical Tensions. The findings also include the Basic Social Process (BSP) of Decision making in a context of risk, along with the final Grounded Theory (GT) of: Wicked Complexity in Paramedics' care for people who Self-Harm. The final chapters at the end of this thesis involve discussions and conclusions, reflecting the methodological outcomes and limitations, implications of its findings for policy, practice, education and directions for future research. 


\section{Chapter Two - Context of paramedic care for those who Self Harm}

\section{Introduction}

Before considering paramedics' perceptions of caring for people who $\mathrm{SH}$, it is important to recognise the context for the care that is provided by paramedics. Whilst it is beyond the reaches of this thesis to provide detailed anthropological insights into the development of paramedics as a cultural group, a consideration of the contextual issues that affect the way care is provided, how they are perceived by those they care for, their development as a group, and response to changing societal needs, will provide the reader with an appreciation of some of the complexities paramedics face when caring for people who SH.

In this chapter a discussion is presented on the move towards clinical effectiveness, governance, and evidence-based care in ambulance services, and their role in the development of United Kingdom (UK) ambulance services and the paramedic profession. The historical context of the development of paramedics is considered, reflecting their origins which stemmed from dealing in military conflict, and how this influenced the trajectory of paramedic and ambulance service care, to initially focus on trauma and life-threatening emergencies through to the advent of technology, drugs and equipment, and an increasing focus on the development of advanced life support for life threatening problems such as out-of-hospital cardiac arrest. With a changing case mix, members of the profession increasingly have had to manage minor illness or injury and psychosocial presentations such as people who $\mathrm{SH}$, those with chronic diseases, and mental health problems. The chapter explores how current policies, legislation and guidelines are increasingly reflecting this modern context of paramedic care, and the reader is presented with details of these policies, legislation and guidelines, along with a discussion of their implications for ambulance services and paramedics.

\section{Current prehospital care provision: Within the United Kingdom and internationally}

There are two broad models for Emergency Medical System (EMS) staffing in different parts of the world: the Anglo-American model and the Franco-German 
model. The Anglo-American model uses non-physician EMS units. This model is largely driven by the higher staffing costs and availability of physicians compared to Emergency Medical Technicians (EMTs) and paramedics, who are increasingly better trained and equipped to deliver high quality care. In contrast, the FrancoGerman model relies on physicians, and emphasizes a high degree of on-scene stabilisation prior to transportation to hospital. The Franco-German model is also utilised in many areas of South America, particularly in Argentina, Chile, Uruguay, and Brazil (Al-Shaqsi 2010). The UK has traditionally followed the Anglo-American model, relying on non-physicians such as EMTs and paramedics to staff ambulances.

Models of ambulance staffing will undoubtedly impact on care for people who SH, due to differences in expertise between doctors, paramedics and EMTs in assessing people with mental health needs, and the transferability of any research in this area should recognise such differing contexts of EMS provision. Education, skills, competence and even the availability of pharmacological agents which may be used to restrain individuals following $\mathrm{SH}$ are some examples of potential differences in physician versus non-physician models of EMS provision. Mental health legislation also differs across the world, which may also influence care in areas such as powers of detention of people who SH.

UK Ambulance Services are increasingly resisting characterisation as either FrancoGerman or Anglo-American, as a range of medical and allied health professionals now deliver services, and models of care are emerging which include mental health nurses and General Practitioners (GPs) working together in ambulance control rooms, or joining police, ambulance and mental health triage teams, which are then deployed by ambulance services (National Audit Office 2017, NIHR 2016).

\section{The development of the paramedic role}

The roots of modern paramedics and ambulance services lie in the battlefields of the Crimean war, which saw the formation and organisation of ambulances and medical attendants, dedicated solely to the care of the wounded. Since this time UK ambulance staff and paramedics have evolved their role; where early ambulance attendants in the UK were once only required to hold a driving licence and offer 
vocational first aid (Kilner 2004), today sees a greatly expanded range of medical care provision by paramedics, supported by national standards in education and training. These are laid down by a range of organisations such as the Health \& Care Professions Council (HCPC) and the College of Paramedics (CoP), which are respectively their professional registration body and professional college.

A series of significant events occurred in UK ambulance service provision which supported the development of UK paramedics. This included the Ministry of Health Committee $(1966 a$; 1966b) report on the equipment and training of staff in the UK NHS ambulance service, which recommended a basic training programme lasting eight weeks for all emergency ambulance staff. The course included first aid training along with other general and technical subjects, and included learning to care for mentally ill patients and some of the legal aspects associated with this care. After 12 months of experience and satisfactory reviews, the Ambulance Services Proficiency Certificate, also known as the Millar programme and certificate (Ministry of Health 1966a, 1966b), was awarded as the basic qualification to ambulance staff. The Millar (Ministry of Health 1966a, 1966b) programme evolved into the Institute of Health and Care Development (IHCD) ambulance technician programme (IHCD 2000), which was equivalent to its international counterpart, the Emergency Medical Technician (EMT) programme (Pozner et al 2004). During the 1980s and into the 2000s, UK ambulances were mostly staffed by ambulance technicians, however, many locally-based paramedic and extended training schemes began to evolve during the 1980s, that were led by enthusiastic medical staff who formed steering groups in individual health board areas (Carne 1999). In the 1990s these schemes were combined into the National Health Service Training \& Directorate ambulance extended training scheme (NHSTD 1991), and later the Institute of Health and Care Development (IHCD) paramedic programme which then followed (IHCD 2003). The IHCD paramedic programme had a major focus on the management of trauma, resuscitation and life-threatening emergencies, with limited provision for psychosocial, mental health or behavioural presentations (IHCD 2003). 


\section{Higher education of UK Paramedics}

Since the development of the initial UK IHCD paramedic programme, it has been increasingly recognised that the focus of paramedic training and education on major trauma, resuscitation and acute conditions such as cardiac and respiratory emergencies, no longer reflects the case mix that paramedics encounter in their dayto-day practice (Lendrum et al, Kilner et al 2004). It has been estimated that only $10 \%$ of patients in the 999 case mix, for example, have a life-threatening condition (DOH 2005). Pressures on the urgent and emergency care system have increased relentlessly over the past decade, and are no longer confined to the winter months (NHS Confederation 2015). This increased demand has created conditions of increasing and unremitting pressure in the Emergency Department (ED), with ambulance services suffering greater and greater delays at turnaround (the time from arrival at ED to becoming available for another call) (Robertson-Steel 2004). Despite this increase, $43 \%$ of ED attendances conveyed by ambulance are discharged, with over two-thirds of those discharged not needing follow-up treatment (DOH 2009).

In order to meet the needs of patients presenting with urgent or non-life threatening conditions, the Department of Health report Taking Healthcare to the Patient: Transforming NHS Ambulance Services (DoH 2005) recommended that:

\footnotetext{
"Ambulance clinicians should be equipped with a greater range of competencies that enable them to assess, treat, refer, or discharge an increasing number of patients and meet quality requirements for urgent care" (DoH, 2005, pg. 44)
}

This statement supported the shift of paramedic education towards higher education. In 2001, the introduction of the Health Professions Order (Health Professions Order 2001) required UK paramedics to register with the regulatory body, the Health Professions Council, from July 2003. The Health Professions Council Standards of Education and Training (2009) set the entry level to the register as a paramedic at equivalent to Certificate of Higher Education for paramedics. Higher Education Institutions (HEIs) developed paramedic science programmes, through partnership with ambulance services that exceeded the HCPC academic entry level. These programmes initially included a Foundation Degree (FD or FdSc) in Paramedic Science or Diploma of Higher Education (DipHE) in paramedic science. Higher 
education for paramedics has evolved further since conducting this study towards a minimum requirement of a $\mathrm{BSc}$ as the threshold entry onto the HCPC register; this is discussed later in this thesis.

\section{Move towards clinical effectiveness, governance, and evidence-based care in ambulance services}

Along with changes to education for ambulance staff, throughout the 1990s and 2000s a move towards clinical effectiveness, governance, and evidence-based care in ambulance services resulted in an evolution of the direction of UK Ambulance Trusts and the paramedic role. The consultation document A First-Class Service: Quality in the new NHS (DOH 1998) set out the framework for quality improvement and fair access in the NHS, the main components of which were communicated by the NHS Executive Health Service Circular (1999/065 p.3) Clinical Governance: in the new NHS, which included:

- Clear national standards for services and treatments through National Service Frameworks and a new National Institute for Clinical Excellence (NICE)

- Local delivery of high quality health care, through clinical governance underpinned by modernised professional self-regulation and extended lifelong learning

- Effective monitoring of progress through a new Commission for Health Improvement. A Framework for Assessing Performance in the NHS and a new national survey of patient and user experience.

The National Service Framework for Coronary Heart Disease (NSF CHD), published by the Department of Health (NHS Executive 2000) recognised the role of ambulance Services in caring for patients with suspected acute myocardial infarction (AMI). It set out organisational goals and milestones for the care of AMI, and many ambulance Services responded with strategies which included further development of their extended training into paramedic schemes. The NHS research and development programme helped with the production of evidence needed to inform clinical decision-making and service planning.

The role of ambulance Services was recognised in many other National Service Frameworks (NSFs), influencing the direction of ambulance Services and their 
delivery of care, evaluated through research. Examples of other NSFs which referred to the role of ambulance staff included the National Service Framework for Older People (DOH 2001), which advocated that ambulance crews refer older people who fall to community-based care and which resulted in a range of referral pathways for elderly fallers being initiated by ambulance Trusts. Such referral pathways for older people who fall have been evaluated through large scale research studies, such as the SAFER studies by Snooks et al (2004, 2012, 2017a, b). The NSF for (neurological) Long Term Conditions (DOH 2005) influenced the education of paramedics in early recognition of stroke and referral to specialist assessment treatment; this again was informed by research, and followed by large scale studies such as PASTA (Shaw et al 2016) and RIGHT-2 (Ankolekar et al 2012). However, the NSF Mental Health (DOH 1999) failed to mention the evolving paramedic role and ambulance services were only mentioned once, where it was acknowledged that people with mental health problems during out of hours may: 'phone for an ambulance' (DOH 1999 p. 28).

This lack of acknowledgment of the role of ambulance services and their staff has since changed and is discussed later in this thesis. However, when considering the significant influence of policies such as NSF Coronary Heart Disease (2000) over the evolution in the role and education of paramedics, greater recognition in mental health policy of the impact on ambulance services, and the potential contribution their staff could make to the provision of mental health care, may have resulted in better opportunities to influence education and the development of the paramedic role at a time of significant transition and development?

\section{Ambulance services as clinical providers of care}

Health services across the western world continue to face challenges with an ageing population, increases in long-term medical conditions, and changing expectations and demands from patients and the public. In 1997 ambulance services were placed at the forefront of a new NHS modernisation programme (UK Parliament 1997), aiming to ensure that they play a key role in the development of quality systems of healthcare delivery. The NHS modernisation programme (UK Parliament 1997) emphasised the importance of national standards to ensure consistent, high-quality 
care as specified in A first-class service (NHS Executive 1998), and the Health Service Circular: Modernisation of Ambulance Services (NHS Executive 1999) set out the government's view that quality care should be at the heart of the National Health Service, and set out a range of objectives for NHS ambulance services. These objectives included new plans for commissioning of emergency and urgent ambulance services, the requirement to have call prioritisation systems in place to identify immediately life threatening (Category A) 999 calls and achieve the required response time targets for all 999 and urgent ambulance calls. NHS ambulance services were also encouraged to work on joint emergency service pilots, and with local clinicians to set up and evaluate pilot studies of alternative ways of responding to minor emergency calls which take account of the clinical needs of patients. The Health Service Circular: Modernisation of Ambulance Services (NHS Executive 1999) recognised the need for these pilots to be agreed by the local health community and carefully explained to the public. The importance of gathering the evidence and evaluating the pilots was also highlighted in order to identifying best practice to inform future policy and allow dissemination within ambulance services.

Ambulance services have continued to develop the vital role they play in addressing these challenges to ensure all patients get the right care, in the right place, at the right time, by fitting their work around the emergence of new ambulance services and becoming main providers of care to the population. This expansion of services has been influenced by many factors, such as increased demand for services and changes in provision for urgent and unscheduled care, yet it must be recognised that a major influence on the recent improvements in clinical standards and the design of services has been the introduction of UK National Service Frameworks.

\section{Future direction of ambulance services and paramedics}

A vision for the future of urgent and emergency care in the UK was presented in the Urgent and Emergency Care Review (Keogh 2013). This positions the ambulance service and paramedics at the centre of care for people not only with life threatening problems, but also those with urgent (non-life threatening) conditions, providing highly responsive, effective, and personalised services outside of the hospital. The Urgent and Emergency Care Review (Keogh 2013) sets out a vision where 
sustainable, high-quality care in hospitals will be achieved in future by relieving pressure on hospital-based emergency services, thus maximising the chances of survival and recovery for people with more serious or life-threatening emergency needs. Ambulance services were called upon to develop alternative approaches to care, such as condition specific pathways of care in order to appropriately reduce ED attendances. This would also require paramedic education, policies and legislation to evolve to achieve this vision.

\section{Guidelines and policy on care for those who Self Harm}

Acknowledging the link between $\mathrm{SH}$ and suicide, the UK Government has sought to reduce suicide through strategies involving collaboration between services, patients and professional groups; these strategies provide guidance and call for more training for health staff caring for people with mental health needs (NICE 2004, NSF Mental Health 1999, The National Service Framework on Mental Health (NSF MH 1999) identified care for those who SH as a key area to meeting a $20 \%$ reduction in suicides by 2010 , and provided details of how such reductions in suicide could be achieved. When considering the impact of such strategies and comparing trends over time it is important to look over a relatively long period, as there may be natural fluctuations year-on-year which may present false increases or decreases that are attributable to any psycho-social predictors (Samaritans 2012). Suicides statistics can give a misleading picture of the prevalence of suicide when considered alone, as rates per 100,000 people are often reported which take into account the effect of population size on the number of suicides (Samaritans 2012). For instance, a group with a larger population may have more suicides than a group with a smaller population, but the rate per 100,000 may be lower. However, a reduction has been seen in the baseline suicide rate in the general UK population of 9.2 deaths per 100,000 population in 1997, to 7.3 deaths per 100,000 population in 2011 (ONS 2014 b.). This general decline in suicide deaths across all ages is shown in Fig 2. 


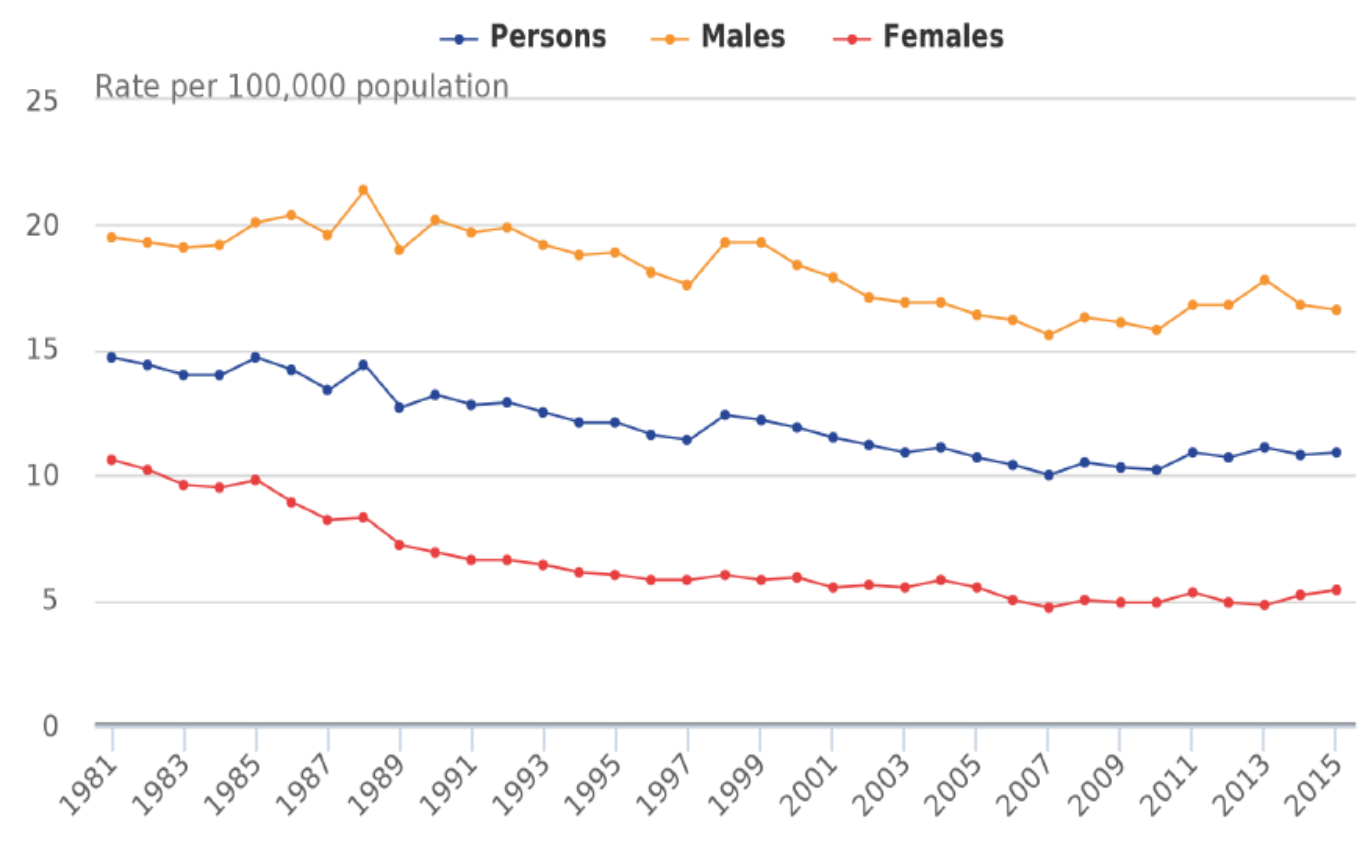

Figure 2: Age-standardised suicide rates by sex, deaths registered between 1981 and 2015 (ONS 2015).

In 2004, the National Institute of Clinical Excellence (NICE) published guidance on SH to advise on the short-term physical and psychological management and secondary prevention of SH in primary and secondary care (NICE 2004). These guidelines were developed by a multidisciplinary group of healthcare professionals, patients and researchers, and were influenced by the best available evidence. The guidelines were intended for use by clinicians and commissioners of services intent on providing and planning care for those people who $\mathrm{SH}$, while also emphasising the importance of the experience of care for service users and carers.

The NICE (2004) Guidelines on SH recognise that people who have self-harmed, their friends and their relatives frequently turn to the ambulance service for help. They recognise that ambulance staff are increasingly better trained in providing care and treatment at the scene and during transportation to hospital for patients with diverse medical conditions, and are in a privileged position to give early treatment and psychological support for patients following SH. NICE (2004) also acknowledges that ambulance staff often have access to the person's home 
environment, and can and gain insights from family and friends not present during hospital treatment on events leading up to the incident of SH.

NICE (2004 p.29) recommend a range of key aims and objectives in the treatment of SH. These are presented in more detail in Appendix A. They include: the need for rapid assessment of physical and psychological need (triage); effective engagement of service users; effective measures to minimise pain and discomfort; timely initiation of treatment; rapid and supportive psychosocial assessment (including risk assessment and comorbidity), and prompt referral for further psychological, social and psychiatric assessment and treatment when necessary; and an integrated and planned approach to the problems of people who self-harm. NICE (2004 p.48) also set out key priorities for implementation, which are provided on more detail in Appendix B, and include the need for respect, understanding and choice, staff training, effective triage, and an assessment of risk.

NICE (2004) recognises that:

"Ambulance staff have an increasingly important role in the assessment and early treatment of self harm, a role that needs to be well supported through effective collaboration with other professional groups" (NICE 2004, p15).

The recommendations from NICE (2004 p.55) relating to ambulance staff are presented in Appendix C. They apply many of the general principles in caring for SH set out above. They call for ambulance staff to urgently establish the likely physical risk, and the person's emotional and mental state, in an atmosphere of respect and understanding. These recommendations call for ambulance staff to be trained in the assessment and early management of $\mathrm{SH}$ and how, if following $\mathrm{SH}$ the service user does not require emergency treatment in the ED, ambulance staff should consider taking the service user to an alternative appropriate service, such as a specialist mental health service, and that the decision to do so should be taken jointly between ambulance staff, the service user and the receiving service. NICE (2004) also provide clear guidance to ambulance services, EDs and mental health trusts on the need to work in partnership to develop locally agreed protocols for ambulance staff to consider alternative care pathways to emergency departments for people who have self harmed. 
Since the publication of the NICE (2004) SH guidelines, the role of ambulance staff in the care of people who SH has appeared frequently in guidelines. RCPsych (2006) echoes many of the points raised in relation to ambulance staff in NICE (2004) guidelines. These recommendations are summarised in Appendix D, and again call for ambulance services to work with other organisations to develop care pathways including service users being taken directly to mental health units, primary care, crisis intervention teams or to social services. RCPsych (2006) suggest that ambulance services, the ED and mental health trusts should develop locally agreed protocols for alternative care pathways for people who have self-harmed. They also call for ambulance staff to have access to telephone advice from crisis resolution teams, approved doctors and social workers regarding the assessment of mental capacity and the possible use of the Mental Health Act (1983).

The Joint Royal Colleges Ambulance Liaison Committee (JRCALC) provides clinical oversight and expert clinical advice to UK Ambulance Services, and produces a set of nationally applicable evidence-based clinical practice guidelines, which are regularly reviewed and updated. JRCALC gathers the available evidence in areas of ambulance service and paramedic care, which is discussed by a team of academics, clinicians and ambulance service representatives. The guidelines provided by JRCALC offer support and advice to paramedics and ambulance services, informed by the best available evidence. However, organisations such as the College of Paramedics have previously criticised JRCALC for their poor referencing of such evidence (Woollard \& Furber 2010). It has long been recognised that much of what is currently believed about pre hospital and paramedic care is based on custom and tradition rather than sound scientific evidence (Lemonick 2009), and Callaham (1997 p231) described the situation as: "The scanty science of pre hospital care" (p.231). It is therefore important that care for people who SH is based on good evidence in order to provide clinically and cost-effective care.

JRCALC has published five updated sets of clinical guidelines in recent years covering paramedic practice, which include aspects of care for people who $\mathrm{SH}$ (JRCALC 2000, 2004, 2006, 2013, 2016). As these guidelines have evolved, they increasingly reflect the recognition of the role of paramedics in the care for people 
who SH. JRCALC (2000) provided limited guidance in relation to SH. Advice was provided on medically managing the physical consequences of $\mathrm{SH}$, such as dealing with the effects of poisoning, overdoses and care of wounds. There was limited information on the psychosocial aspects of $\mathrm{SH}$, such as why people $\mathrm{SH}$, what patients think of care after SH and the link between SH and completed suicides. JRCALC (2000) did however, provide a discussion on SH in relation to consent. They recognised that cases of $\mathrm{SH}$ presented challenges for health professionals such as paramedics. JRCALC (2000) advised that where a patient can communicate, an assessment of mental capacity should be made as a matter of urgency. If the person lacks mental capacity, JRCALC (2000) advised that they must be treated in their best interests unless there was an existing living will. However, JRCALC (2000) advised that if the patient has capacity and refuses treatment, the patient's GP should be contacted urgently to fully assess their level of capacity. They added that if the incident was more critical and there was insufficient time, crews should act more formally and in the patient's best interests as they currently acted rather intuitively, using documentation to assess whether they perceive the patient to be at risk of suicide. They advocated the use of a suicide assessment form (Fig 3) for this, which they advised may be of value in assessing some mental health patients

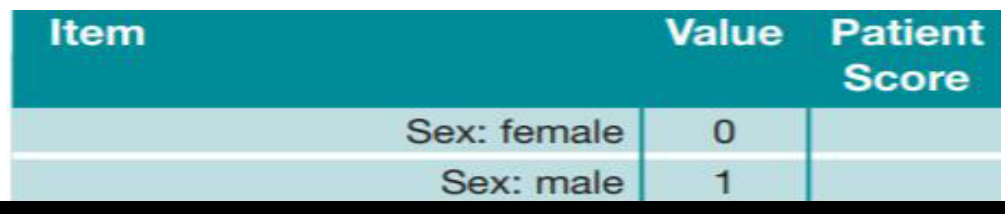
who either lack mental capacity or rationality. This suicide checklist was included in the 2013 JRCALC guidelines (JRCALC 2013), which were current when the research reported in this thesis first started.

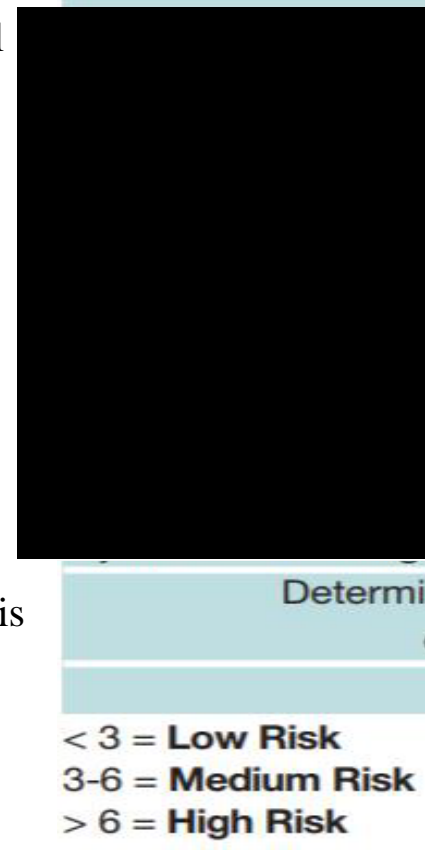

Fig 3 JRCALC (2006 p.191) Suicide and Self-harm Risk Assessment Form 
Paramedics have traditionally been taught how to conduct a psychosocial assessment within their training and education, but nevertheless may increasingly be using such risk assessment tools advocated by JRCALC which are commonly used in ED. Risk assessment tools are checklists of risk factors, symptoms or antecedents, but evidence for their effectiveness is limited (Hawley et al 2006 NICE 2006). Quinlivan et al (2014) found that a wide range of non-validated tools were in use among EDs and mental health services which, they suggested, demonstrates little consensus over the best instruments for risk assessment and reflects the style of service provision in that setting and a 'high risk' approach to management. Quinlivan et al (2014) also found that mental health staff were less likely to use published risk scales, reflecting a greater reliance on comprehensive psychosocial assessment. Whilst such assessment forms may be of assistance to paramedics in the care of people who $\mathrm{SH}$, RCPsych (2010) suggests that the prediction of suicide, and the assessment of suicide risk in respect of any individual patient is virtually impossible, and such tick box assessment mentally "removes staff from people, devalues engagement and impairs empathy...empathic listening and talking have key therapeutic benefits" (RCPsych 2010 p79). Overreliance on assessment tools leads to complacency, they suggest, and can misdirect people away from a detailed history-taking and mental state assessment (RCPsych2010). Assessment in which the patient's views are taken seriously and where they are encouraged to participate in decisions about their care and treatment, and which have clear explanations for decisions taken, are highly rated (Taylor et al, 2009). Paramedics may therefore for a long time have been using non-validated risk assessment tools in the care for people who $\mathrm{SH}$, with limited capacity to provide the psychosocial assessment called for by NICE (2004) for all people who present following SH. This reflects some of the limits of paramedic care for people who $\mathrm{SH}$, such as the need for collaboration with other professional groups who can provide an effective psychosocial assessment as called for by NICE (2004).

Throughout this thesis, it is consistently recognised that paramedics are often the first professionals to encounter people who SH. Paramedics encounter people who threaten to harm themselves, engage in $\mathrm{SH}$ or die by suicide, yet few researchers have sought to investigate their care for this patient cohort. In order to improve care in this encounter, it is important to understand $\mathrm{SH}$, what $\mathrm{SH}$ is and what it is not, so 
that subsequent care and treatment is appropriate to the needs of the person. The many definitions of self-harming behaviour were explored, and whilst the RCPsych (2010) definition is presented in this thesis, the challenges and limitations of such definitions are recognised. The Royal College of Psychiatrists (2010) hold that SH is a behaviour trait, a manifestation of emotional distress, and an indication that something is wrong, rather than a primary disorder. Such factors therefore have implications around the assessment of $\mathrm{SH}$ as a mental health problem, and application of legislation such as the Mental Capacities Act (2005) and Mental Health Act (1983).

The factors associated with $\mathrm{SH}$, what motivates a person to $\mathrm{SH}$ and wider issues which influence $\mathrm{SH}$, are also important considerations to improving how paramedics respond to people who SH. Guidelines recognise many of these factors, but to make meaningful improvements in the care for people who $\mathrm{SH}$, recognising this is not enough. There needs to be an understanding from the paramedic perspective why people SH and how multiple and complex issues influence their delivery of care. These multiple issues may include social and demographic factors in $\mathrm{SH}$, historical factors influencing $\mathrm{SH}$, psychological, psychosocial, motivation and intent of $\mathrm{SH}$, the nature of the act of $\mathrm{SH}$, the link between $\mathrm{SH}$ and suicide; these issues and many more have potential to influence paramedic care for people who SH.

\section{Mental Health Legislation relevant to paramedic care for people who Self Harm} In the England and Wales two pieces of legislation are of fundamental importance in the management of SH. These are the Mental Health Act (MHA 1983) and the Mental Capacity Act (MCA 2005). NICE (2004) recognises that in the pre hospital setting those who SH may refuse treatment. In the UK, the Joint Royal Colleges Ambulance Liaison Committee Paramedic Guidelines (JRCALC 2006) suggest that when a patient can communicate; an assessment of their mental capacity should be made urgently, and that if they lack capacity they must be treated in their best interests unless there is an existing living will. However, if the patient has capacity and refuses treatment, the patient's GP should be contacted urgently to fully assess their level of capacity. 
Following SH, all patients should have a full bio psychosocial assessment carried out by a skilled and experienced clinician (RCPsych 2010, NICE 2004) which assesses a range of factors such as personal circumstances, social context, mental state, risk and needs, and is central to the clinical management of SH. Kapur (2003) found that receiving psychosocial assessments can halve the risk of repetition of $\mathrm{SH}$, yet fewer than half of SH patients attending ED have been reported to have received assessment (Kapur 2003, NICE 2004).

Many who present to paramedics and police following SH and are at risk of further $\mathrm{SH}$, suicide, or injury to themselves and others, refuse to attend ED for such assessment and treatment (Rees et al 2016, IPCC 2015). In such circumstances the England and Wales Mental Health Act and Mental Capacities Act (MHA 1983, MCA 2005) are of relevance. The MCA (2005) upholds the right of an individual to make their own decisions and advocates a functional approach to decision-making around capacity that involves establishing the extent to which an individual's abilities meet the demands of a particular decision. Measuring such capacity requires the patient's ability to make decisions as well as:

- Understanding information relating to the specific decision

- Using the information to make a choice

- Communicating that choice.

The MCA (2005) reflects the Shared Decision Making (SDM) approach which has been advocated in UK NHS policy for several years (Coulter et al 2017a, National Voices 2013). Following a recent landmark ruling in Montgomery $v$ Lanarkshire Health Board (Coulter 2017b) SDM became a legal imperative throughout the UK, requiring that people with full mental capacity must be properly advised about their treatment options and the risks associated with each option so that they can make informed decisions when giving or withholding consent to treatment (Coulter et al 2017 a.). Paramedics therefore have an ethical, legal and professional responsibility (HCPC 2017) to apply the principles of SDM and MCA (2005), this is unless the UK Mental Health Act (MHA 1983) applies (NICE 2004). The MHA (1983) sets out when a person can be admitted, detained and treated in hospital against their wishes. The Sections used in emergency detention of people who SH are summarised below: 
- Section 4: Is applied in emergency situations to detain a person for up to 72 hours in the interests of their own health and safety or to protect other people. An application for admission must be made by an Approved Mental Health Professional (AMHP) or a nearest relative with recommendation of one doctor.

- Section 135 (a): an AMHP can seek a warrant from a magistrate, to allow a police officer, the AMHP and a doctor to enter premises and remove a patient to 'a place of safety' for assessment.

- Section 135 (b): a justice of the peace may grant a warrant allowing a police officer to force entry to a premises in order to search for a person who is 'absent without leave' or 'liable to be detained'. Officers may then use other powers under the MHA (1983).

- Section 136 is applicable if:

"A constable finds in a place to which the public have access a person who appears to him to be suffering from mental disorder and to be in immediate need of care or control, the constable may, if he thinks it necessary to do so in the interests of that person or for the protection of other persons, remove that person to a place of safety." (Sec. 1361983 MHA)

Since publication of the Mental Health Act 1983, there have been many policy documents which have supported and developed its application. The legislation itself has also evolved throughout the duration of this study, which culminated in changes to the Sections above of the MHA (1983) through the Police and Crime Act (2017).

In 2008 the Mental Health Act 1983: Code of Practice was introduced (Mental Health Code of Practice 2008). It is statutory guidance for registered medical practitioners and other professionals in relation to the medical treatment of patients suffering from mental disorder. As there have been substantial changes and updates in legislation, policy, case law, and professional practice, this code was revised in 2015 to reflect and embed developments in areas including the use of restrictive interventions, seclusion, use of police powers to detain people in places of safety, and the use of community treatment orders (Mental Health Code of Practice 2015). The Mental Health Code of Practice (2015) provides statutory guidance to registered medical practitioners, approved clinicians, managers, providers of care, other staff and approved mental health professionals on how they should carry out functions under this Act in practice. 
The Mental Health Code of Practice (2015) calls for ambulance services to ensure they have in place a clear joint policy for the safe and appropriate admission of people in their local area, agreed at board or board-equivalent level by each party, and that each party should appoint a named senior lead. The Code advises that those involved in such local policy should meet regularly to discuss its effectiveness in the light of experience, and review the policy where necessary, to decide when information about specific cases can be shared for the purpose of protecting the person or others, in line with the law. Those carrying out functions for these parties should understand the policies and their purpose, the roles and responsibilities of other agencies involved, and follow the local policy and receive the necessary training to be able to carry out fully their functions. In terms of SH, the intention of the Mental Health Code of Practice (2015) is to protect patients, and particularly those at risk of suicide and SH. However, it is recognised that any arrangements should also aim not to impose any unnecessary or disproportionate restrictions on patients or to make them feel as though they are subject to such restrictions.

In December 2017, amendments to Sections 135 and 136 of the Mental Health Act came into force following changes to the Police and Crime Act (2017). These changes were designed to ensure police officers can act faster and more flexibly, whilst ensuring that people receive the assessment and treatment they need in a timely manner. The changes also now allow for a person to be kept at a place of safety (and not solely removed for a mental health assessment), if it is appropriate and they consent.

The Police and Crime Act (2017) changes included the definition of public place, by identifying the following places where police cannot exercise their powers under Section 136:

a) any house, flat or room where that person, or any other person, is living, or;

b) any yard, garden, garage or outhouse that is used in connection with the house, flat or room, other than one that is also used in connection with one or more other houses, flats or rooms.

Other than these excluded areas, the police will be able to exercise their powers under Section 136 anywhere which will facilitate them to act quickly to protect 
people found in places such as railway lines, offices and rooftops which have previously not necessarily been considered as places to which the public have access.

Another important change introduced by The Police and Crime Act (2017) is that, where practical to do so, the police have an added duty to consult a registered medical practitioner, a registered nurse or an approved mental health professional, before deciding to remove a person to or to keep them at a place of safety. The amendments also make clear that a suitable private property (with consent of the occupier) is an appropriate place of safety. A place of safety may therefore be a person's own home or other places such as community centres or other multiple use buildings. The new addition of Section 136A prevents the use of police stations as a place of safety for under $18 \mathrm{~s}$ and seeks to limit the use of police cells as places of safety for adults and increase the safeguards in place where a police station is used as a place of safety for an adult. Section 136A also permits the Secretary of State to make regulations regarding the use of police stations as places of safety and may in future include provision for regular review and availability of appropriate medical treatment.

Important changes around timings of detentions were also introduced through the Police and Crime Act (2017). The maximum period for detention to allow for a mental health assessment under Section 135 and Section 136 changed from 72 hours to an initial maximum period of 24 hours, which commences from the time when the person arrives at the place of safety or the time a police officer enters the property if he/she subsequently decides to keep the person at that place. At the end of the 24hour period an extension of up to 12 hours may be granted by the registered medical practitioner responsible for the examination of the patient. A more detailed presentation of these changes provided by Brown (2018) can be found in Appendix (E).

Along with legislation and guidance documents on the application of such legislation, there is overarching strategy and policy which has implications for caring for people who SH. In 2014 the UK Department of Health published the Mental Health Crisis Care Concordat: Improving outcomes for people experiencing mental health crisis (Mental Health Crisis Care Concordat 2014). The aim of the concordat was for collaboration and improved care in a crisis for people with mental health 
problems, however the concordat resulted in joint statements, written and agreed by signatories, describing what people experiencing a mental health crisis should be able to expect of the public services that respond to their needs. The concordat serves as a joint statement of intent and common purpose, and of agreement and understanding about the roles and responsibilities of each service; it is intended to ensure people who need immediate mental health support at a time of crisis get the right services when they need them, and get the help they need to move on and stay well. Ambulance services and paramedics feature frequently in the Mental Health Crisis Care Concordat (2014), which also involves a wide range of partners including health and social care, commissioners, the police and local communities. The vision of the Concordat recognises the role of paramedics in providing initial assessment to people in mental health crisis, whilst acknowledging concerns, and that services do not always respond well, stating:

"Every day, people in mental health crisis situations find that our public services are there when they need them - the police officers who respond quickly to protect people and keep them safe; the paramedics who provide initial assessment and care; the mental health nurses and doctors who assess them and arrange for appropriate care; and the Approved Mental Health Professionals, such as social workers, who coordinate assessments and make contact with families." (p.6)

And:

"These services save lives. There is much to be proud of. But we must also recognise that in too many cases people find that the same services do not respond so well. There have long been concerns about the way in which health services, social care services and police forces work together in response to mental health crises." (p.6)

The Concordat reinforces how emergency staff should treat people who have selfharmed which is in line with the NICE (2004) guidance. As with the NICE (2004) guidelines, the Mental Health Crisis Care Concordat (2014) again emphasises how screening, following SH, should determine a person's mental capacity, their willingness to remain for further psychosocial assessment, their level of distress, the possible presence of mental illness and their need for referral for appropriate psychological therapies and follow-up. Further detail is also given in the concordat on what to expect when a decision is made by a police officer to use their power under Section 136 of the MHA (1983), and how it is essential that the person in crisis is screened by a healthcare professional as soon as possible. Recognising that 
ambulance staff are often the first health professionals in contact with somebody following $\mathrm{SH}$, the concordat recognises that in most cases it will be the ambulance service that will screen the person to exclude medical causes or complicating factors and advise on the local healthcare setting to which the person should be taken.

The Mental Health Crisis Care Concordat (2014) provides specific guidance for ambulance services. This includes making sure there is provision for round-the-clock advice from mental health professionals within the clinical support infrastructure in each ambulance control room. Enhanced levels of training for ambulance staff are called for on the management of mental health patients; this could be multi-agency training to ensure a truly joined up approach. The concordat also provides examples of good practice, which includes a Section 136 working group made up of the police, a hospital trust, the ambulance service, Approved Mental Health Professionals (AMHPs) and the local authority. This group created a joint mental health process which fully outlined operational protocols and responsibilities. Their protocol included a central Section136 number for the police to use to enable them to access information and support from mental health professionals.

\section{Summary}

This chapter explored the broad and changing context of paramedic care for people who SH. The differences in prehospital care provision and models for EMS staffing in other parts of the world were discussed. It was highlighted that in the UK, where this study was conducted, ambulance staffing has traditionally followed an AngloAmerican model, relying on non-physicians such as EMTs and paramedics to staff ambulances. This context was important to recognise due to differences in expertise between doctors, paramedics and EMTs in assessing people with mental health needs, and the transferability of any research in this area. Despite this it was recognised that, including paramedics and EMTs, a wide range of medical and allied health professionals now work in ambulance services, and these include mental health nurses and GPs working together in ambulance control rooms, or joint response initiatives with police and others. 
The development of the paramedic role and organisation of ambulance services were presented, and whilst these developments began from the battlefields with ambulance staff providing care of the wounded, since this time the UK paramedic role has evolved into an autonomous professional role regulated by the Health \& Care Professions Council (HCPC) and with its own professional college, the College of Paramedics $(\mathrm{CoP})$. Throughout this development it has been increasingly recognised that the focus of paramedic training and education on major trauma and lifethreatening emergencies no longer reflects the case mix that paramedics encounter in their day-to-day practice, as only $10 \%$ of patients in the 999 case mix have a lifethreatening condition. Paramedic development therefore continues to evolve to meet this changing case mix with paramedics now being developed through higher education paramedic science programmes.

Along with changes to education for ambulance staff, throughout the 1990s and 2000s there was a move towards clinical effectiveness, governance, and evidencebased care in ambulance services driven by policy documents such as A First-Class Service: Quality in the new NHS (DOH 1998) and the Health Service Circular: Modernisation of Ambulance Services (NHS Executive 1999). Policy documents such as these recognised that ambulance trusts/services play a key role in the development of quality systems of healthcare delivery, which need to be based on good evidence, and which started to emerge in documents such as National Service Frameworks.

The National Service Framework on Mental Health (NSF MH 1999) identified care for those who SH as a key area to meeting a $20 \%$ reduction in suicides by 2010 . Whilst the National Service Framework for Coronary Heart Disease (NSF CHD) and others recognised the role of ambulance staff and shaped the development of the paramedic role, the NSF Mental Health (DOH 1999) failed to mention the evolving paramedics role, and the only reference to ambulance trusts/services was that people with mental health problems may phone for an ambulance. Despite this, reductions have been seen in suicide rates in the general UK population following NSF MH (1999). However, since publication of the NSF MH (1999) a wide range of guidelines and policies have made detailed reference to the role of paramedics and 
ambulance trusts/services in providing early treatment and psychological support for patients following SH (Mental Health Crisis Care Concordat 2014, NICE 2004, RCPsych 2006), and along with such general policy and guidance (JRCALC 2000, 2004, 2006, 2013, 2016), also include specific guidance for paramedics in the care of $\mathrm{SH}$, which has also evolved over time.

A key aspect of these guidelines includes the need for people who SH to receive a psychosocial assessment; such assessment can halve the risk of repetition of $\mathrm{SH}$, yet fewer than half of SH patients attending A\&E have been reported to received assessment (Kapur 2003, NICE 2004). Paramedics have traditionally not been taught how to conduct psychosocial assessment within their training and education, and therefore this must be conducted by another appropriately skilled health professional. Suicide risk assessment tools are however advocated by JRCALC, and a wide range of such non-validated tools are commonly used in ED. Despite this, RCPsych (2010) advises that overreliance on assessment tools leads to complacency and can misdirect people away from detailed history-taking and mental state assessment.

All of the recent policies and guidelines in England and Wales focussing on the care for people who SH (Mental Health Crisis Care Concordat 2014, NICE 2004, RCPsych 2006, JRCALC 2000, 2004, 2006, 2013, 2016) highlight the fundamental importance of the Mental Health Act (MHA 1983) and the Mental Capacity Act (MCA 2005). The MCA (2005) upholds the right of an individual to make their own decisions, and that decision-making around capacity involves establishing the extent to which an individual's abilities meet the demands of a particular decision.

Measuring such capacity requires the patient's ability to make decisions as well as to understand information relating to the decision, and to use the information to make a choice and communicate that choice. The MCA (2005) reflects the Shared Decision Making (SDM) approach (Coulter et al 2017a, National Voices 2013) which requires that people with full mental capacity must be properly advised about their treatment options and the risks associated with each option so that they can make informed decisions when giving or withholding consent to treatment (Coulter et al 2017 a.). Paramedics therefore may encounter people with varying levels of mental capacity and have a legal and professional responsibility to adequately assess their mental 
capacity, and should the person lack capacity, the paramedic may be required to act in the patient's best interest.

Elements of the MHA (1983) relevant to the care of people who SH were also presented, which set out when a person can be admitted, detained and treated in hospital against their wishes, and the range of options in care decisions. It was discussed that Section 136 of this Act is sometimes applied by the police in the emergency detention and removal to a place of safety of a person following $\mathrm{SH}$, where it is in the interests of that person or for the protection of other people. 


\section{Chapter Three - Research Design: Methodology and Methods}

\section{Introduction}

It has previously been recognised in this thesis that following $\mathrm{SH}$ a paramedic is often the first health professional contact. Despite this, there is limited published literature or evidence providing understandings of this encounter. A small number of studies have focussed on the patient's perspective, and they report the quality of care and attitudes from these staff are unsatisfactory. Understanding this care from the provider's perspective may yield greater in-depth understandings of why this is the case, the multiple influences on care, and ultimately provide opportunities to improve services.

The aim of the present study was to explore paramedics' perceptions of caring for those who SH in order to inform education, policy and practice. The objectives were: Objective 1: To reveal paramedics' perceptions of caring for those who SH Objective 2: To develop a theory on paramedics' perceptions of caring for $\mathrm{SH}$

Health Services research is increasingly drawing on qualitative research methods to gain insights and understandings, as they are well suited for uncovering links between concepts and behaviours, understanding phenomena within their context, and generating and refining theory (Bradley et al 2007). In relation to SH, qualitative methods can provide understanding of paramedic care of this patient group, and paramedics' perceptions of the act of SH. The study used Evolved Grounded Theory Methodology (EGTM) (Strauss \& Corbin 1990, 1998, Charmaz 2000). Grounded Theory Methodology (GTM) was first conceived by Glaser and Strauss (Glaser \& Strauss, 1967) and is an inductive, theory-discovery methodological approach, which can provide researchers with a detailed understanding of what people do, their prime concerns, and how they deal with these concerns (Crooks 2001). GTM developed along two methodological paths, the traditional GTM of Glaser and Strauss (Glaser \& Strauss, 1967), and the evolved form of GTM (EGTM) used in this study, which follows a relativist ontology (Mills et al 2006). The relativist ontology reflects how the world consists of multiple individual realities influenced by context, and that concepts such as truth, reality, right, good, or norms must be understood as: 
"relative to a specific conceptual scheme, theoretical framework, paradigm, form of life, or society, or culture" (Bernstein, 1983, p. 8 In Mills et al 2006).

In this chapter the methodology and methods are presented, including a discussion on the theoretical perspective of the study. The literature review encompassed a systematic review of the quantitative literature and a metasynthesis of the qualitative literature, followed in this chapter by a discussion of the literature review and its place in EGTM and the implications of the constructivist perspective of EGTM on the literature review. This is then followed by a discussion of GTM, its evolution into EGTM, and why it was used in this study. Further details are then provided of the methods employed in the study, including the rationale for sampling, selection of participants, data collection, data analysis, considerations of transparency and trustworthiness through reflexivity and member checking, and finally the ethical considerations and limitations.

\section{The literature review and its place in EGTM}

There is much debate over the timing of the literature review in EGTM (Heath 2006, McGhee et al 2007). Glaser and Strauss (1967) acknowledged that the researcher will not enter the field free from ideas, but their views differed in relation to the role of literature, and in particular the need to conduct an initial review. Glaser (1978) viewed the researcher's role as that of expert, suggesting prior in-depth study of the literature may violate the emergent nature of qualitative research with pre-conceived ideas. This tension between emergence and forcing is at the heart of the debate between the need for reflexivity and the positioning of the literature review (Glaser 1992). Glaser \& Strauss (1967) agreed that is necessary to approach the area of study with more than 'general wonderment', however Strauss (1987) developed the notion that the use of self and the literature are an early influence, because while diffuse understandings provide sensitivity, both the literature and specific understandings from past experience may be used to stimulate theoretical sensitivity and generate hypotheses (Heath \& Cowley 2004). Strauss and Corbin (1994) suggested that researchers should reflect critically on themselves and their behaviour, as well as continuing to positively emphasise the researcher's familiarity with the literature associated with the research area in such a way that it might be played against systematically gathered data. 
EGTM is a way of generating new theory, grounded in the field but also set in the context of existing theory (McGhee, 2007), and Strauss \& Corbin (1990) advocate reviewing the literature early in a study for the following reasons:

- It provides a secondary source of data.

- It stimulates questions.

- It stimulates theoretical sensitivity.

- It directs theoretical sampling.

The methodological and philosophical justification for the use of EGTM has previously been discussed, however the utility and implications of EGTM continue to require attention when reviewing the literature. Questions emerge as to the relationship between the knower and what is known? How do we know what we know? And what counts as knowledge? A critical issue relating to the construction of the literature review and evolving theory in this study was that paramedics' perceptions of caring for those who SH involves a phenomenon occurring in the social world and, for that reason, a constructivist social science approach has been followed, which queries what therefore is the value of the literature review? And what understandings can be abstracted from studies published? In order to address these questions, the underlying philosophical and methodological stances in relation to the literature review required consideration.

The influence of research philosophy in the literature review in this study includes important assumptions about how the researcher observes or views the social world. Beliefs about the nature of reality, humanity, and the theory of knowledge that informs the research all affect the methods in which a researcher thinks about the research process (Bahari 2010). Tuli (2010) contends that consideration of the epistemology, ontology and methodology must be a central feature of any discussion about the nature of social science research as these elements give shape and definition to the conduct of an inquiry.

Epistemology is a branch of philosophy that studies the nature of knowledge and what constitutes acceptable knowledge in the field of study (Saunders et al 2007). It is concerned with the nature, validity and limits of inquiry (Rosenau 1992). Epistemological assumptions are associated with the methods through which 
knowledge can be acquired. Ontology refers to the nature of reality; it is the claim or assumption that a particular approach makes about the nature of the reality under investigation (Blaikie 1993). There are two broad epistemological positions: positivism and interpretivism-constructivism. In the interpretivist-constructivist perspective, the theoretical framework sees the world as constructed, interpreted, and experienced by people in their interactions with each other and with wider social systems (Lincoln \& Guba 2000). Positivism however assumes there are social facts with an objective reality apart from the beliefs of individuals (Bahari 2010). Positivist research methodology is considered 'objective' or detached, where the emphasis is on measuring variables, using experimental designs, testing hypotheses that are linked to general causal explanations, and providing evidence in a quantitative form (Sarantakos 2005). The present study, by using qualitative methodology, was underpinned by the interpretivist-constructivist epistemology and relativist ontology, which acknowledge the importance of a multiplicity of perspectives and "truths" (Strauss, 1987; Strauss \& Corbin, 1990, 1994, 1998). This assumed that meaning is embedded in the participants' experiences and that this meaning is mediated through the researcher's own perceptions (Merriman 1998).

EGTM constructivist methodology holds that reality is subjective, multiple and socially constructed by its participants (Krauss 2005, Lincoln \& Guba 2000), viewing patterns of behaviour as being created out of evolving meaning systems that people generate as they socially interact (Neuman 2003). The focus of the constructivist researcher is to concentrate on what people think and feel, how they communicate with each other (verbal or non-verbal) and to attempt to understand and explain why people have different experiences; the researcher's role is to appreciate/interpret the different constructions and meanings based on people's experience (Bahari 2010). By this study following a constructivist epistemology of EGTM, a prescribed flexible design has been adopted in which the researcher's view of reality has influence over all other assumptions. The literature review recognises that areas of literature will contain measurable facts and present established evidence; however, it also accepts that this will be an incomplete exploration of knowledge and literature in this area due to the complexity and multiple influences on paramedics' perceptions of care for those who SH. 
Deduction and verification dominate analysis in the Strauss and Corbin (1990) approach, which recognises that familiarity with relevant literature can enhance sensitivity to subtle nuances in data (Strauss and Corbin 1998 p.49). Care was therefore taken to avoid confirming existing knowledge rather than discovering new knowledge, by inspiring the need to develop a theory free of the methodological and conceptual pitfalls of previous studies, whilst also bridging the perceived gaps in existing knowledge.

The previous section explored the context within which the study takes place. It highlighted how paramedic care for people who SH has many potential influencing factors. The increasing number of people who $\mathrm{SH}$, the many who avoid services, negative attitudes, or fear of detention under the MHA, all make it a complex area of study. However, a raft of guidelines point to the potential contribution paramedics can make in the care of those who $\mathrm{SH}$, and how such care should be supported by education.

The overall aim of the literature review was to explore and identify what was known at the time about paramedic care for those who SH and inform the development of theory on paramedics' perceptions of their care. Paramedic practice has traditionally lacked an evidence base due to its over reliance on hospital-based practices and those of other professional groups. Callaham (1997) recognised this, describing paramedic practice as "the scanty science of pre hospital care" (p1.), and whilst the evidence base has improved significantly since this statement, it was anticipated that there would be few published papers specifically relating to paramedic care for those who $\mathrm{SH}$. The literature review therefore recognised how these factors relating to paramedics' perceptions of care for those who SH are broad and complex, and reviewing the literature subsequently held several functions; it was exploratory, a method of mapping out areas of uncertainty, identifying whether the proposed study had already been conducted, and where it sat in the body of research. It also attempted to start to develop theoretical insights within the constructivist approach to the study. In order to meet such broad aims, a rigorous approach was employed which recognised established knowledge in the care for those who $\mathrm{SH}$, gained 
through both quantitative and qualitative methods. This was achieved by a systematic review of the quantitative literature and metasynthesis of the qualitative literature.

An initial systematic review of the quantitative literature was conducted, exploring perceptions of emergency care staff of those who SH. This systematic review included literature from emergency staff including paramedics, doctors and nurses, as it was anticipated that there would be limited literature exclusively exploring paramedics' perceptions. The aim of the systematic review was to systematically review published quantitative literature relating to paramedic and emergency care staff perceptions and experiences of caring for people who SH. More detail on this systematic review is provided later in this thesis. It was recognised early on that the transferability of findings from literature gained from professional groups such as emergency doctors and nurses would be limited due to differences in areas such as education, skills, setting and context between professional groups and care settings. However, when exploring such a field, where there is little prior information upon which to evolve theory, one must start somewhere, to justify the study and set the detail within a body of research.

A further metasynthesis of the qualitative literature was conducted in this study, which combined insights from a range of methodological approaches, offering the potential for a much richer, more flexible, and dynamic approach to evidence and theory development (Downe 2008). This was in line with the EGTM approach to this study. This review purposefully broadened the focus of the study to reflect the complexity of care for $\mathrm{SH}$ from a paramedic context. The metasynthesis also served to provide a panoramic view of the research question, and to help the researcher understand why paramedics' perceptions of care for those who SH matter.

Sandelowski et al (1997 p.366) defines qualitative metasynthesis as:

"Theories, grand narratives, generalizations, or interpretive translations produced from the integration or comparison of findings from qualitative studies".

The aim of the metasynthesis was to enhance knowledge building and theory generation to develop practice and policy through a metasynthesis of qualitative 
research relating to perceptions of paramedic and emergency care for people who $\mathrm{SH}$. Further details on the metasynthasis are provided later in the thesis.

The EGTM approach is iterative and integrative, with data collection, analysis and conceptual theorizing occurring in parallel and from the outset of the research process (Duhscher \& Morgan 2004). The literature review continued as the study progressed to ensure all relevant papers were considered, and through 'memoing', reflexivity and constant comparison method, the interplay between the emerging theory, the literature and categories developed were monitored, and continued until the theory generated explained any variation (Benton 2000). These techniques will be described in detail later in this chapter.

\section{Grounded Theory Methodology (GTM)}

GTM was first conceived by Glaser and Strauss, and published in The Discovery of Grounded Theory: Strategies for Qualitative Research (Glaser \& Strauss, 1967).

This work offers a research approach with an inductive, theory-discovery methodology, allowing the researcher to develop a theoretical account of the general features of a topic, while simultaneously grounding the account in empirical observations or data. GTM gives a picture of what people do, what their prime concerns are and how they deal with these concerns (Crooks 2001) and is therefore used to explore the social aspects of human interactions (Struebert \& Carpenter 1999). Application of GTM can develop explanations of key social processes or structures that are derived from, or grounded in, empirical data.

\section{Evolution of GTM}

Soon after Glaser and Strauss's (1967) seminal text, they developed GTM along two different methodological paths. Glaser followed what is referred to as Classic GTM, or a critical realist (or traditional) method, which is accepted as being faithful to the original formulation by Glaser \& Strauss (1967). Strauss however pursued a relativist ontological (or evolved) form of GTM, corresponding with his central concern that action is at the heart of both the process of and structure of data collection and analysis (Corbin 1991). McCann \& Clark (2003) encourage us to understand the evolution of GTM as a spiral of methodological development, as opposed to a 
situation of binary opposition. A key aspect to the evolution of grounded theory in EGTM is its constructivist methodology and relativist ontology, which acknowledge the importance of a multiplicity of perspectives and "truths" (Strauss, 1987; Strauss \& Corbin, 1990, 1994, 1998). Key to Strauss's version of GTM was his assumption that there is not one truth, but that the external world is a symbolic representation, where both the interior and exterior worlds are created and recreated through interaction (Strauss 1993) and there is no divide between external and interior world. Strauss (1993) positions the researcher as the author who reconstructs meaning through the research process, acknowledging that the researcher and the researched co-create the theory; this recognises the influence of macro-social factors on action, and accepts that reality cannot be fully known but is interpreted and is linked to time and place. This is consistent with a relativist ontology that pays attention to the broader environmental and contextual factors (macro conditions) that influence the phenomenon under study. Strauss follows a constructivist paradigm of inquiry where the researcher is a "passionate participant as facilitator of multi-voice reconstruction" (Lincoln \& Guba 2005 p. 196). Mills et al (2006) refer to the work of Strauss \& Corbin $(1990,1998)$ as an evolved form of (EGTM) and this is the form of GTM applied to the present study. GTM (Glaser and Strauss, 1967) and EGTM (Strauss and Corbin, 1998; Charmaz, 2006) both have the following key features of theoretical sensitivity, the constant comparative method of data collection and analysis and theoretical saturation.

\section{Role of the researcher in EGTM}

A key role of the researcher in EGTM is to develop theoretical sensitivity, which involves the ability to give insight and meaning to data, (McCreaddie \& Payne 2010). For Glaser (1978), the researcher's role is one of expert and conceptual innovator, and whilst the researcher becomes sensitised to the literature, Glaser warns against seeking out literature that is not already to hand. Theoretical sensitivity is considered a way the researcher guards against potential biases that can be a threat to the rigour of the study. As noted earlier, EGTM follows the constructivist perspective that acknowledges people construct the realities in which they participate, highlighting the researcher-participant dyad and the co-construction of data (Charmaz 2006). McCreaddie \& Payne (2010) illustrate this role of the 
researcher in EGTM in their study into the phenomenon of spontaneous humour in healthcare interactions. The previous experience of the author as a stand-up comedian gave insight and awareness of the subtleties of the data and the ability to bring meaning to data in line with Strauss and Corbin (1990).

Theoretical sensitivity is a multidimensional concept that includes the researcher's level of insight into the research area, how attuned they are to the nuances and complexity of the participant's words and actions, their ability to reconstruct meaning from data generated with the participant, and a capacity to "separate the pertinent from that which isn 't"' (Strauss \& Corbin, 1990, p. 44). The EGTM approach used in this study fundamentally differs from classic GTM, where Glaser (1967) claims that the researcher must be a tabula rasa, or blank slate, when entering a field of inquiry (Clarke, 2005) to develop theoretical sensitivity legitimately. The relativist ontological position of Strauss and Corbin (1994) leaves behind this traditional subscription to the discovery of truth that emerges from data representative of a real reality (Glaser 1978). Rather Strauss and Corbin (1994) reject the existence of a "pre-existing reality 'out there", as to think otherwise is to take a positivistic position that ... we reject ... Our position is that truth is enacted" (p. 279). The main researcher in this study was a practicing paramedic and this background was important as theories themselves are "interpretations made from given perspectives as adopted or researched by researchers" (Strauss \& Corbin, 1994, p. 279).

Theoretical sensitivity was cultivated in this study via a comprehensive literature review and metasynthesis. Additionally, by virtue of previous experience as a paramedic, the researcher had insight and awareness of the subtleties of the data and the ability to bring meaning to the data (Strauss and Corbin, 1990). It was recognised, however, that in such qualitative research, personal preconceptions, including those of the researcher, may inhibit the process of discovery within healthcare contexts (Holloway 2005). To counteract the impact of preconception or presupposition on the process of study involvement, a biosketch of the researcher is presented below and in Appendix F describing his background and involvement in 
the research and any implications of his work role, and other associations with the project.

\section{Biosketch}

In qualitative research, it is suggested the researcher should avoid over-influencing data with pre-conceived ideas they have from previous projects or other life experiences (Jasper 1994). Authors suggest that one's preconceived ideas may inhibit the process of discovery from research data (Holloway 2005). The biosketch early in this study presents the researcher's past, assumptions and experiences in this area and is continuously referred to in order to consider the influence of prior knowledge on this study's data capture activities. However, in EGTM, there is also a recognised need for the researcher to draw on and harness past experience in a positive context, to ensure the researcher and participants co-construct theory and knowledge is built together (Charmaz 2006).

The paramedic researcher in this study (NR) undertook this exercise before any data were collected, describing how he had been a paramedic for twenty-five years, holding positions supporting education and clinical practice and providing advanced care in the ED as an Advanced Emergency Practitioner. The researcher had therefore cared for many people who $\mathrm{SH}$, witnessed death by suicide because of $\mathrm{SH}$ and observed the care of other paramedics throughout this time. The biosketch (appendix F) was helpful in supporting the researcher's reflections on the data and data analysis, and assisted the researcher in overcoming any negative influences of personal preconception to add transparency to research processes and to illuminate the researcher's decision trail as the study progressed. Such background has the potential for preconceived ideas and previous encounters to influence the construction of the EGTM, however, it also adds to theoretical sensitivity which includes:

"the researcher's level of insight into the research area, how attuned they are to the nuances and complexity of the participant's words and actions, their ability to reconstruct meaning from the data generated with the participant, and a capacity to separate the pertinent from that which isn 't"' (Strauss \& Corbin, 1990, p. 44). 
Prior experiences of the researcher in the present study was therefore considered a positive influence and is vital to the constructivist approach of EGTM being applied.

\section{Why EGTM for this study}

When embarking on a new investigation, Struebert \& Carpenter (1999) challenge the Evolved Grounded Theorist to ask whether empirical research and the published literature have offered what seems to be an oversimplification of the concepts relevant to the phenomenon under investigation? Whilst there is much written on $\mathrm{SH}$ based on other professional groups, it was recognised that scant attention has been paid to the interaction of SH patients with paramedics. The very nature of paramedic practice is built on the premise of saving life, and despite paramedics often being the first point of contact for those who threaten to conduct or manage to $\mathrm{SH}$, leading in some cases to severe injury and even death, this area is yet to be thoroughly investigated. The vital role that this first contact plays in the care for those who SH was highlighted by the Royal College of Psychiatry (2010), yet it cannot be assumed that interactions and investigations on other professional groups and care settings can be transferred to the paramedic population when faced with those who SH. Adopting Strauss and Corbin's (1998) EGTM codified procedures to the present study benefited from interplay between the participants, the researcher, data and emerging theory on paramedics' perceptions for caring for those who SH.

It was previously argued that application of EGTM can develop explanations of key social processes or structures that are derived from, or grounded in, empirical data. The social process in the present study is the care that paramedics give to $\mathrm{SH}$, and key to understanding this care, will be the illumination of paramedics' perceptions of this interaction. An in-depth inquiry of the basis of these perceptions, what they were and why they were formed, sought to generate explanations which can influence this social process and structure of care through education, policy and practice.

\section{Research methods}

\section{Sample selection}

The study was conducted between 2014 and 2016 in one UK ambulance service, covering a population of three million people. A poster requesting volunteer 
paramedics for the study was distributed to the whole ambulance service area.

Following responses to the poster call, potential participants were selected using theoretical sampling methods, based on the characteristics of age, gender, years of paramedic experience, rural or urban setting, and educational development. These characteristics were identified by a systematic review of the quantitative literature and metasynthesis of the qualitative literature conducted by the researcher, both of which are reported later in this thesis. Following the poster call, study participants were contacted by email, thanking them for volunteering to be involved in the study.

Theoretical sampling is a fundamental aspect of GTM and essential to the development, refinement and grounding of theory in data (Struass \& Corbin 1998). Theoretical sampling involves collecting further data in the light of categories that have emerged from earlier stages of data analysis. In theoretical sampling, emerging theory is checked against reality by sampling incidents that may elaborate or challenge the evolving claims. Despite applying an evolved form of grounded theory in this study, the importance of theoretical sampling remains, as described by Glaser \& Strauss (1967):

"the process of data collection for generating theory whereby the analyst jointly collects, codes and analyses his data and decides what data to collect next and where to find them, in order to develop his theory as it emerges" (Glaser and Strauss 1967 p.45).

Study participants were theoretically sampled throughout the study and were invited for interview based on their characteristics reflecting areas of enquiry being constructed. As data emerged during coding and analysis of interviews, the researcher selected participants who it was felt would yield rich insights into the areas of interest emerging in interviews. Interviews continued until no new information was forthcoming where a state of saturation was deemed to have been achieved.

Prior to the interviews a suite of documents was prepared which included a participant information sheet, consent form, document capturing demographics and interview guide (Appendix G). All of these documents were reviewed as part of the WAST Research Risk Review and the NHS research approvals process, and were 
localised to include the WAST crown badge and logos. Sampled participants were provided with a pack including a consent form and information sheet about the research.

The validity, meaningfulness and insights generated from qualitative inquiry have more to do with the information richness of the informants selected than the sample size (Potton 1990). Therefore, as well as having experience of attending to patients who $\mathrm{SH}$, informants had to be willing to critically examine their own experiences in a reflective manner. Two pilot interviews were undertaken, with one paramedic working in an urban area and one paramedic based in a rural area. It was anticipated that this sampling approach could yield rich patterns emerging from geographical variation, as well as capturing the core experiences, disparate issues and central shared aspects that cut across the service variation. The interview guide was amended following the pilot interviews and data from these interviews were included in the study proper. Subsequent participants for the study were sampled based on emerging concepts in the data, to include participants with attributes more likely to reveal and explore insights on the emerging data. Two of the participants were interviewed twice to further explore insights revealed from initial interviews, and seven agreed to follow-up on their interviews through member checking, which is a way of finding out whether the data analysis is "congruent with the participants' experiences" (Curtin \& Fossey 2007 p. 92). This approach was in line with Strauss and Corbin (1998) who emphasis how theoretical sampling is a means to: "maximise opportunities to discover variations among concepts and to densify categories in terms of their properties and dimensions" (p.201).

The earlier stages required maximum openness and flexibility to identify a wide range of predominantly descriptive concepts. As these concepts emerged through analysis of the data, participants in the study proper were theoretically sampled to further reveal and explore insights within the emerging data and to construct categories and develop the evolving Grounded Theory. Participants in the study proper were initially sampled to reflect variation in experience, educational development and where they were based. This was due to initial coding revealing concerns over limited training and preparation for paramedics in caring for people 
who $\mathrm{SH}$. As data analysis evolved during the study process, these opportunities were maximised to compare events, incidents or happenings as previously described. Consequently, subsequent participants were theoretically sampled to explore emerging concepts and sharpen the focus of the research aims, as advocated by Strauss \& Corbin $(1990,1998)$. Informants were deliberately sought to include those who were likely to generate data of more relevance to the emerging concepts. In the latter stages, sampling became more discriminating, testing the emerging theories, for instance, in deliberately seeking out deviant cases or how well the emerging hypothesis held up in different settings. This approach to sampling continued throughout the study in order to achieve saturation of codes, refinement and construction of the categories, and ultimate development of the Evolved Grounded Theory.

Guba \& Lincoln (1989) recommend sample selection to the point of redundancy, to maximise information; a state of saturation is then said to exist (Munhall 2001). Glaser and Strauss (1967 p. 65) first defined such a state of saturation as the point at which:

"no additional data are being found whereby the (researcher) can develop properties of the category. As he sees similar instances over and over again, the researcher becomes empirically confident that a category is saturated... when one category is saturated, nothing remains but to go on to new groups for data on other categories, and attempt to saturate these categories also" (Glaser and Strauss 1967 p. 65)

Despite the need for saturation, it was recognised in the present study that the research proposal required stating up front the number of participants to be involved. Guest et al (2006) recognised such challenges of individuals designing research in terms of how many interviews they should budget for and write into their protocol, before they enter the field. Guest et al (2006) documented the progression of category identification in their study, examining perceptions of social desirability bias (SDB) and accuracy of self-reported behaviour in the context of reproductive health research. Guest et al (2006) found that data saturation had occurred by the time they had analysed twelve interviews. Based on this, saturation in the present study was predicted to occur following analysis of twelve interviews; a sample size of between twelve and twenty-five paramedics was therefore anticipated. The 
sampling was terminated when no new information was forthcoming, and saturation was the primary criterion for the sample size decision-making of when to stop, which eventually resulted in eleven interviews being conducted.

The sampling evolved throughout coding and analysis. The analysis is discussed in more detail later in this chapter and followed Strauss and Corbin's (1998) three levels of open, axial and selective coding. The first stage of open coding entailed comparing data for similarities, differences and questions regarding emergent phenomena, resulting in identification of indicators, which are words or phrases of interest to the area of study. Indicators were then subsumed under higher level headings known as concepts, which stand for the emerging phenomena. The second stage of axial coding involved categorisation by taking concepts and thinking of how they could be further formulated into higher-level headings known as categories. The third stage of selective coding was an "explication of the story line" (Strauss \& Corbin 1998, p. 148) which involved identifying the basic social process (BSP) at work, around which all other categories revolved. Finally, theoretical constructions conceptualised the relationship among the three levels of coding, by weaving the fractured data back together again to form the Evolved Grounded Theory.

It was noted that there was a potential relationship with the sensitive concept and busyness and urban paramedic practice. It was therefore decided to use busyness and urban paramedic practice as a proxy to the sensitive concept and actively sample and over recruit participants in busy urban areas to maximise the potential of gaining greater insights into the issues relating the sensitive concept.

\section{Development of the interview guide}

The purpose of the interview was described by Schwandt (2000) who tells how researchers use interviews to stimulate conversations with participants about the meaning of their experiences. An interview guide approach was used, which consisted of semi-structured questions to cover the broadly defined agenda that arose from the research questions and literature review. The literature review and metasynthesis revealed a significant research gap in that no research had explored paramedics' perceptions of care for people who SH. The literature review and 
metasynthesis did however provide areas of enquiry which consistently emerged from studies involving other health and emergency care providers, and also research exploring wider paramedic care for mental health emergencies where SH was discussed. Categories from the metasynthesis and systematic review informed the development of the interview guide. The interview guide explored what paramedics thought about the care they provided for people who $\mathrm{SH}$, as individuals and as a professional group. Paramedic participants were asked for examples of cases they felt went well, where they perceived a high standard of care was provided, and also where care provided was not helpful to the patient, or they perceived a low standard of care was provided; they were asked to talk through these cases. The interview guide explored if the paramedics perceived the care they provided was appropriate and, if not, what difficulties or barriers they encountered? Finally, they were asked if they were sufficiently informed and supported to deliver a high standard of care for people who $\mathrm{SH}$, and had an opportunity to raise any other issues.

The interview guide, whilst structured, allows for flexibility in enabling respondents to digress (Gall, Gall, \& Borg 2003). It ensured that the same general areas of information were collected, whilst also giving participants the opportunity to describe experiences in detail, giving their perspectives and interpretation of these experiences. The interview guide therefore allowed for exploration and probing of accounts, but there was containment of the dialogue to an extent.

As initial data were gathered and analysed, they led to refinement of the study's central focus and thus to new questions for participants (Glaser \& Strauss, 1967). As categories emerged, following data analysis of early interviews, emergent issues were included in subsequent interviews and others discarded, thus cross-validating findings. Theoretical sampling was previously discussed in more detail in sample selection, however on-going data analysis and theoretical sampling based on categories emerging out of interviews influenced the interview guide, allowing for rich exploration of emergent concepts and theory development. 


\section{Data Collection}

\section{Interviews}

A one-hour, face-to-face, one-to-one, semi-structured interview took place with all participants using a semi-structured interview guide. This gave informants the opportunity to describe experiences in detail, providing their perspectives and interpretations of these experiences and allowed for a deeper exploration and probing of accounts, but also giving a certain containment to the participant dialogue. As categories emerged, following data analysis of early interviews, emergent issues were included in subsequent interviews, thus cross-validating findings.

Interviews were conducted at a location and time convenient to participants. The first two pilot interviews assisted in determining if there were any flaws, limitations or other weaknesses with the design of the interview guide. This also allowed for necessary revisions to the interview design and schedule prior to implementation for the study proper. Interviews were audio-tape recorded with notes taken, and audiorecordings were transcribed verbatim by the researcher and checked for accuracy against the recordings to validate the hard copy data and ensure it was a close match to the raw material.

Silverman (2006) warns that when embarking on a qualitative interview the researcher needs to be aware of the situation and culture in which the respondent is located, with advice to be polite, display courtesy and facilitate talk without judgement or critical opinion in order to establish trust. The EGTM approach to this study and the researcher's professional background as a paramedic enhanced this connection and engagement with the language and culture of the respondents in a way that was felt helped to gain a level of trust. It was necessary to rapidly develop a positive relationship during the interviews, and building rapport was essential to this process. It is the key ingredient in qualitative interviewing (King \& Horocks 2010). Blohm (2007) views rapport building as a degree of acceptance or cooperation on the part of the interviewee to a research project and, as such, a relationship was sought that was based on trust and a respect for the interviewee and the information shared. This was enhanced by establishing a safe and comfortable environment for sharing the interviewee's personal experiences and attitudes. 


\section{Coding}

\section{Data Analysis}

The study did not set out to test an existing hypothesis, rather it set out to generate theory from the research situation in the field as it was. EGTM both discovers and relates concepts. Based on the initial research question, aims and objectives, and with the information from the literature search, data analysis in EGTM achieves theory generation through an inductive-deductive interplay approach. Induction is viewed as the key process in EGTM, with the researcher moving from the data to empirical generalisation and on to theory. Strauss and Corbin (1998) advocate such induction via on going data comparison, where emergence is ensured by deduction followed by verification and elaboration from further data comparison; the researcher shapes data by interpretation, moving analysis beyond description (Fig 4).

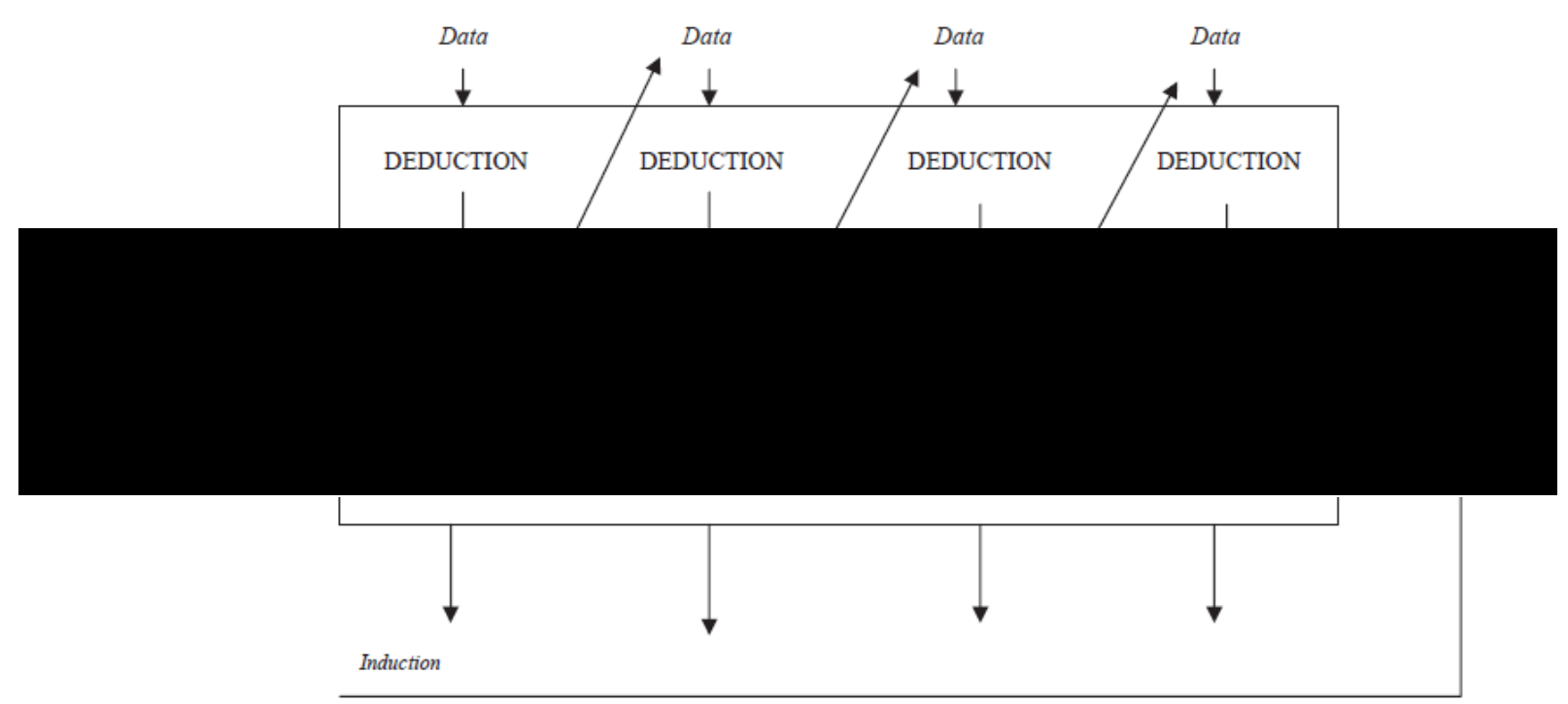

Fig. 4 Strauss and Corbin (1998) induction, deduction and verification.

This EGTM approach both discovers and relates concepts and the paramedic researcher's role in data collection and analysis reflects the constructivist perspective of EGTM, which assumes that people construct the realities in which they participate, and thus highlights the researcher-participant dyad and the coconstruction of meaning (e.g. Charmaz, 2006). Whilst the paramedic researcher entered the field open to realising new meaning through cycles of gathering data and analysis, he progressively focused on integrated concepts. Strauss and Corbin (1998) 
advised that such coding processes need to be used with a degree of flexibility and creativity, an approach that is reflective of its constructivist intent.

Transcriptions were read line by line, and coded through the three levels of open, axial and selective coding below. NVIVO (2015) V10 software was used in this process. Coding was not a linear process and required simultaneous collection, coding and analysis of data, asking of the data what is happening and what does it represent? The aim was to identify categories, relationships between and within categories and a central phenomenon around which all other categories will revolve. The researcher was the instrument of data collection and analysis, and Strauss (1993) positions the researcher in EGTM as the author who reconstructs meaning in the research process, acknowledging that the researcher and the researched co-create the theory. The researcher is a "passionate participant as facilitator of multi-voice reconstruction" (Lincoln \& Guba 2005 p. 196). Corbin and Strauss (2008) are clear that researchers should trust their instincts and not focus too closely on the analytical procedures suggestion:

"Sometimes, one has to use common sense and not get caught up in worrying about what is the right or wrong way. The important thing is to trust oneself and the process. Students should stay within the general guidelines ... and use the procedures and techniques flexibly according to their abilities and the realities of their studies." (Strauss and Corbin, 1998 a p.295)

Transcriptions were coded by NR using constant comparison through the following levels of coding:

- Open Coding: Data were compared for similarities, differences and questions regarding emergent phenomena, in line with EGTM (Struass \& Corbin 1998 p.102). The concept-indicator model (Strauss 1987) was used in this process. An indicator refers to a word, phrase or sentence, and a concept is a label associated with that indicator. Open coding also involved formulation of concepts which “stand for a phenomenon" (Strauss \& Corbin 1990 p. 101).

- Axial Coding: A second stage of coding involved categorisation which involved taking concepts and thinking how they could be subsumed under a higher-level heading.

- Selective Coding: Defined as: "explication of the story line" (Strauss \& Corbin 1998, p. 148). This entails intense analysis around categories (Strauss 1987), 
seeking to answer questions about when, where, why, who, how and with what consequence? Selective coding also involves identifying the Basic Social Process (BSP), which relies on higher level abstraction to identify one overarching emergent social process which can explain variation in all lower level categories, concepts and processes.

- The BSP is central to the grounded theory explaining the social phenomenon under investigation, the nature of which is the subject of the derived theory (Glaser, 1992; Glaser \& Strauss, 1967). The BSP helped conceptualise and explain the social processes that influenced paramedics' perceptions of care for people who $\mathrm{SH}$. This was done across all of the categories using reflective memos and returning to the literature in order to describe the underlying social processes.

- Finally, theoretical constructions conceptualised the relationship among the three levels of coding, by weaving the fractured data back together again.

NVIVO (2015) v.10 software was used in the analysis and coding process to support a thorough examination of data and to enable the researcher to return to the analysis framework to refine and hone the emerging theory, as and when necessary. Strauss and Corbin (1998) point out it had not been their intention to promote rigidity and insist the procedures they outline are “... guidelines, suggested techniques but not commandments" (p.4), and to "... use the procedures in their own way" (2008). Strauss and Corbin (1990) argue that explanation is only partly grounded in the data and that the rest is derived from investigator interpretation. Ideas and potential insights began to develop which were recorded in reflective analytical memos. These memos included theoretical notes about the data and their conceptual connections which supported the development of the theoretical sensitivity. The findings of the study were then compared with the existing evidence and brought to the next level of data conceptualisation in order to answer the research question.

Transparency and trustworthiness were observed by a second researcher who was a speech and language therapist and who independently reviewed the coding framework and then discussed the framework with the primary researcher. Reflective notes were made by both researchers which considered theory development and 
monitored the researchers' influences on this process. In addition, the primary researcher returned on a second occasion to all study participants to check their response to the researcher's own understanding of meaning-making, using 'memberchecking' techniques. Using member checking, the data categories being constructed were shared with participants by presenting them with the coding book for their consideration. Participants were asked to consider the findings emerging and the construction of categories and any misunderstandings that arose from this process were reviewed and returned to participants for clarification.

\section{Demonstration of rigor}

It has been discussed throughout this thesis how EGTM follows an interpretivistconstructivist epistemology and relativist ontology, which contrasts with positivist research methodology involving experimental designs, providing evidence in a quantitative form. Validity and rigour in research in the positivist paradigm can be ensured by activities such as statistically testing correlations between data and ensuring a statistical significance. There are no such equivalents in qualitative and constructivist research as these involve social constructions of knowledge and induction into the area in order to deduce, rather than objective deductive measure as in positivism. Lincoln and Guba (2000) argue that qualitative research cannot be judged on the positivist notion of validity, but should rather be judged on an alternative criterion of trustworthiness. Strauss \& Corbin (1998) call for the Grounded Theory researcher to ask themselves if all the parts of the theory fit with each other and if they appear to explain the data. The criterion of credibility is presented below to consider this question:

A study is considered credible, suggest Guba and Lincoln (1989), when it presents such faithful interpretations that people having the experience would recognise it as their own. Throughout this proposal an acknowledgement is made of the intersubjective construction of data as in qualitative research, "the researcher is the instrument" (Patton, 2001, P. 14). An awareness of the impact of the researcher's previous life experience, including previous reading, was tended to by memo-writing and such 'turning back' helped the researcher to become aware of the potential effects of this on the data. Reflexivity through memoing in a journal aided the 
researcher in becoming more analytical and reflective as time passed (Munhall 2001). Robson (2002, p. 22) states that reflexivity is:

"An awareness of the ways in which the researcher as an individual with a particular social identity and background has an impact on the research process".

Reflexivity therefore helped in monitoring the interaction of the researcher with the participants as well as the process of inquiry, which further enhanced credibility (Holland 2005). Examples of such memo writing and reflexivity are provided in more detail in the results chapter.

\section{Considerations of transparency and trustworthiness through reflexivity and member checking}

In this work, the researcher is a relative insider in the field, studying a topic that is already reasonably familiar. By using Evolved Grounded Theory Methodology, this study harnessed the opportunity for the participants and the researcher to co-create theory. There were researcher's reflective notes which considered the researcher's influence on areas of the emerging theory. A method known as member checking was also employed to find out whether the data analysis was congruent with the participants' experiences (Curtin \& Fossey, 2007, p. 92). In keeping with member checking, findings from coding were shared back with participants for them to consider if they recognised the interpretations and emerging theory. More detail is provided in the analysis and results chapters on how the developing coding book was shared with participants both electronically by email and as a hard copy in one interview. These efforts at transparency in the analysis were supported by prolonged engagement with the data, reflexivity and testing the emerging interpretation against participants' perspectives.

\section{Ethical considerations}

As the study involved NHS employees, research permission was sought from the National Institute for Social Care and Health Research (NISCHR) permissions cocoordinating unit (PCU) and through the Integrated Research Application System (IRAS), as advocated by NISCHR (2011). Whilst completing the NHS Integrated Research Application System (IRAS) application the researcher was directed to consider whether the study required Research Ethics Committee (REC) review. The 
Proportionate Review Service (PRS) was utilised in this process and an outline of the project summarising its purpose, methodology, type of participant and planned location was submitted through the NRES Queries Line. The researcher was advised that the study did not require REC review and was directed to the NRES flowchart “does my project require review by a Research Ethics Committee?” (NRES 2011a).

Research governance was observed by submitting a full proposal of the study to the Welsh Ambulance Services NHS Trust (WAST) Research \& Development Office. Appendix $\mathrm{H}$ is the result of these considerations and outlines the permission granted. The expressed position of WAST following deliberations over the need for REC review, concurred with NRES (2011a) that REC review was not required due to the study involving interviews of NHS staff recruited as research participants by virtue of their professional role.

Despite not requiring REC review, there still remained issues of an ethical nature which need tending to in the study. The Economic and Social Research Council (ESRC 2010, updated 2012) provides a framework to support research being carried out to a high ethical standard. They advocate addressing the following six key principles of ethical research whenever applicable:

1. Research should be designed, reviewed and undertaken to ensure integrity, quality and transparency.

2. Research staff and participants must normally be informed fully about the purpose, methods and intended possible uses of the research, what their participation in the research entails and what risks, if any, are involved. Some variation is allowed in very specific research contexts for which detailed guidance is provided in.

3. The confidentiality of information supplied by research participants and the anonymity of respondents must be respected.

4. Research participants must take part voluntarily, free from any coercion.

5. Harm to research participants and researchers must be avoided in all instances.

6. The independence of research must be clear, and any conflicts of interest or partiality must be explicit.

$\operatorname{ESRC}(2012$ p.2-3)

Whilst ethical standards were observed through the application of the ERCS (2012) framework, due to the iterative process of EGTM it was recognised that immediate 
judgements would be made on the ground about unanticipated events and opportunities which may fall outside the formal specification of how the study should be conducted. This situation has also been recognised as possible by ERCS (2012), which states:

"Not all risks can, or in some cases, should be avoided, but it is important that RECs and researchers develop awareness of potential risks. Such risks may be difficult or impossible to quantify or anticipate in full prior to the start of a social science research project, especially in longitudinal, qualitative research" (ERCS 2012 p.27)

Pels (1999) called for a 'morality of negotiation' in qualitative research, advocating tolerance and recognition of 'emergent ethics', composed contingently, not always consistently, but appropriately and pragmatically for the situation in hand. Such a view calls for a shifting away from formal processes of external regulation and towards increased reliance on, and recognition of, the training, skill and trustworthiness of the individual researcher (Murphy \& Dingwall 2007). Ethical deliberation in this context was therefore informed by understanding why and how decisions were made in specific contexts, rather than specifying a priori rules on how they should be made. Pollock (2012) points out that:

"This is not to say that research ethics is then left rudderless on a sea of relativism and descriptive subjectivity, nor that researchers should not be subject to scrutiny and supervision”. (P.8)

Researchers nevertheless, should endeavour to determine possible risks and their management (ERCS 2012) and Pollock (2012) argues that a more appropriate approach to protecting the ethical integrity of qualitative research is through recognition of the distinctive epistemological and methodological paradigms involved, calling for the application of 'micro' or 'process' rather than 'procedural' ethics. Micro ethics, Pollock (2012) says, is based on judgement rather than rules, relying on the cultivation of 'ethical mindfulness' on the part of the researcher.

EGTM depends not just on the willing cooperation of respondents, but also on their engagement and collaborative input; it is in the process of engagement between researcher and participant that 'data' are generated and the research can be accomplished. Pollock (2012) advocates such participation be based on trust and motivation during which consent is confirmed as an on-going process and validated 
by continuing involvement.

ESRC (2012) encourages the research community to share guidance, experience and solutions to ethical dilemmas to facilitate innovative research. Compared with health professionals engaged in research, social scientists appear to have paid far more attention to ethical aspects of qualitative research. The British Sociological Association Statement of Ethical Practice (BSA 2004) does not provide a set of recipes or choices for resolving ethical dilemmas or choices, but recognises that it will be necessary to make such choices on the basis of principles and values and often the choices of those involved. The BSA (2004 p. 1-7) framework encourages researchers to address the following ethical issues when designing and conducting studies:

- Professional integrity

- Relations with and responsibilities to research participants

- Relationships with research participants

- Covert research

- Anonymity and confidentiality

- Relations with and responsibilities to sponsors or funders

- Clarifying obligations roles and rights

- Pre-empting outcomes and negotiations about research

- Obligations to sponsors and funders during the research process British Sociological Association Statement of Ethical Practice (BSA 2004 p.1-7)

The current study was influenced by a wide body of literature and synthesis of the BSA (2004 p.1-7) and ESRC (2012) guidelines into the following ethical framework:

- Professional integrity, quality and transparency

- Relations with and responsibilities to sponsors or funders, independence of research and potential conflicts of interest

- Clarifying obligations roles and rights

- Pre-empting outcomes, negotiations about research and accurate reporting.

\section{Professional integrity, quality and transparency}

A wider discussion of the complexities and nomenclature surrounding professionalism is presented later and this section seeks not to pre-empt this work. However specific aspects of an ethical nature are discussed. In the first instance, it is the responsibility of the researcher, or research team, to be guided by their professional disciplinary standards (ESRC 2012), and as both the researcher and 
participants were paramedics, the HCPC (2012) standard of conduct performance and ethics could inform the ethical dimension of professional integrity. HCPC (2012) states that:

"You must behave with honesty and integrity and make sure that your behavior does not damage the public's confidence in you or your profession. You must justify the trust that other people place in you by acting with honesty and integrity at all times. You must not get involved in any behavior or activity which is likely to damage the public's confidence in you or your profession" (p.14)

An issue of concern would be if a paramedic had witnessed practice that they perceived as poor or erroneous, raising questions regarding the point at which action would be required, whereby the right to confidentiality needed to be compromised for the sake of a patient's welfare. A priori decisions are difficult to define in such a circumstance, and require a judgement on behalf of the researcher, influenced by factors such as the severity of the incident, or if it had already been addressed in practice. It was made clear from the outset that revealing criminal practices or those representing gross breaches of professional conduct to the researcher would result in professional or police authorities being informed. Whilst no instances required such action in the study, issues of professional integrity, quality and transparency provided significant challenges to the researcher, which are discussed later in the discussion of the results and emergent ethics.

Participation in research should be based on freely given informed consent with a responsibility placed on the researcher to explain in appropriate detail and in terms meaningful to the participant what the research is about, who is undertaking and financing it, why it is being undertaken and how it is to be disseminated and used (BSA 2004, ESRC 2012). According to Johnson and Long (2007) ethical guidelines are typically framed in the language of rules and absolutes. ESRC (2012) cautions however that highly formalised or bureaucratic ways of securing consent should be avoided in favour of fostering relationships, in which on-going ethical regard for participants is sustained, even after the study itself has been completed.

Consent was sought without threat or inducement and it was recognised that issues of power and vulnerability might influence decisions to participate. For instance, being recruited by the paramedic's supervisor may pose obstacles to the process of free and 
informed consent. Polit and Beck (2004) hold that participants enter into a special relationship with researchers and it is crucial that this is not exploited, either overtly or subtly. The context chapter revealed the quasi militaristic development of UK Ambulance Services and how much of the hierarchical command and control structures endure to this day. Along with the potential for coercion, threat or inducement, lasting implications such as career opportunities within these structures gives greater responsibility to the researcher to protect anonymity. The study design ensured as far as possible that participation remained confidential, and care was taken from the point of recruitment where involvement in the study began from a voluntary response to a poster call.

Research staff and participants were informed fully about the purpose, methods and intended possible uses of the research, what their participation in the research entailed, and what risks, if any, were involved. Research participants must take part voluntarily, be free from any coercion and can leave at any time under their own free will. This information was conveyed to participants verbally and through the consent form (Appendix G) and re-affirmed throughout the study.

Participants' anonymity and confidentiality was protected as they were drawn from a sample known only to the researcher. The participants were in the study as people volunteering to be interviewed. BSA (2004) advises that threats to the confidentiality and anonymity of data should be anticipated and it is recognised that the interview venue may threaten anonymity, along with limiting the openness of the interviewee. WAST properties were therefore avoided and mutually agreed venues chosen that were private (within a public building), free from noise and easily accessible to the participant. The location was known well in advance of the interview.

Appropriate measures were taken to ensure research data were confidential and stored in a secure manner in line with the Data Protection Act (1998). Identifiable data such as tapes and transcripts were coded with pseudonyms known only to the researcher. Care was also taken when publishing that any identifiers were removed. It was made clear, however, that Freedom of Information (FoIA 2000) legislation provides the public with a right to access information held by a UK public authority, 
which includes most universities, colleges, or publicly-funded research institutions. The information requested could include research data, which must be provided unless an exemption or exception allows the institution not to disclose it. Should such a request for information be made, the anonymising safeguards in the study design would protect the identity of participants.

\section{Relations with, and responsibilities to, sponsors or funders, independence of research and potential conflicts of interest}

A common interest existed between sponsors, funders and researchers in this study in the advancement of knowledge about caring for people who SH. Although the outcome of the research may have limited benefit and implications for funders, even if the funders are behaving honourably, there may still be perceived pressure on the researchers to deliver a product that pleases the funders so as to ensure funding continues. It is made clear throughout this study that the researcher has obligations to people who $\mathrm{SH}$, research participants, society and the paramedic community (BSA 2004), and this relationship ensures the research is conducted professionally and independently, separate to employing organisations' and funding bodies' desires.

\section{Clarifying obligations, roles and rights}

From a qualitative perspective, informed consent is considered to be an on-going process (Cox et al 2009, ESRC 2012), and participants have the right to decline to take part or withdraw from a study without fear of reprisal. In practice this is not simple as finding the voice to question may be difficult. Neville and Haigh (2003) argue it may be naïve to assume that a no thank you is within the scope of some potential participants, and another crucial issue is the participants' perception of what might happen to them if they do decline (Clark and McCann 2005). Participants had free anonymous access to the researcher by email and telephone in this study and could request a face to face meeting at any time. The researcher was also proactive in contacting participants by email to discuss continued participation in the study and update them on progress.

Whilst SH is a common emergency, asking paramedics to discuss how they perceive their care may involve recalling traumatic cases. Potentially significant emotional, spiritual, physical, social and psychological consequences may emerge for all 
individuals involved in the research and researchers have a responsibility to the wellbeing of the participants (BSA 2004).

Whilst no instances of complaint or harm can be found in the literature in interviews with qualitative health researchers, Dickson-Swift et al (2006) found that researchers often felt more like a counsellor or a friend than a researcher. Subsequently respondents reported research participation to be a positive experience, even when this involved discussion of topics that may be difficult and distressing (Pollock 2012). Despite this, it is recognised that there may be an immediate or delayed impact on the mental health of anyone involved that may include a physical response to the psychological impact of harm or risk. The interview may therefore create issues for all participants in the research process and, whilst some may find the interview useful for counselling reasons, for others it may bring back things that they thought were dealt with and finished. It was anticipated that, during the interview, the interviewer may be required to assume a counselling role, and strategies could include giving the interviewee time to cry or express significant emotion and to acknowledge the importance of this to the wellbeing of the participant. Therefore, in this study, the researcher was conscious of the need to be aware of cues and/or signals by which the interviewee was indicating distress. The researcher allowed the interviewee to terminate the interview if they became too distressed and at the end of the interview a debriefing took place in order to facilitate psychological safety. In addition, the interviewer offered to arrange support through the relevant agencies, in keeping with the anonymous counselling service Network of Staff Support (NOSS: WAST internal staff support network).

\section{Pre-empting outcomes, negotiations about research and accurate reporting:}

No conditions from funders, employers or participants were accepted contingent with a particular outcome or set of findings from the research. The right to publish and disseminate the results of research was also addressed in the request to WAST for permission. A view of candor and frankness in terms of funding was adopted, where openness and transparent reporting was agreed. WAST currently acts as the gatekeepers to paramedics in Wales, as they are the main employer of paramedics, and their approval was required prior to approaching them. Whilst WAST were 
encouraging and supportive in facilitating the research, the researcher was in no way obliged to devolve the responsibility onto WAST in terms of protecting participants anonymity or dealing with psychological and emotional issues which may occur following involvement in the research. Care was also taken not to disrupt the professional relationship between participants in the study and WAST as their employer, as this relationship was recognized as one that would continue long after the research has ended.

\section{Summary}

In this chapter it was discussed how GTM was first conceived by Glaser and Strauss (1967) and offers a research approach that is an inductive, theory-discovery methodology, allowing the researcher to develop a theoretical account of the general features of a topic, while simultaneously grounding the account in empirical observations or data. GTM gives a picture of what people do, what their prime concerns are, and how they deal with these concerns (Crooks 2001). It also discussed how GTM developed along two different methodological paths and that the evolved form of GTM of Strauss \& Corbin $(1990,1998)$ was applied to the present study. EGTM subscribes to the notion that there is not one truth, but that the external world is a symbolic representation, where both the interior and exterior worlds are created and recreated through interaction and there is no divide between the external and interior world. It was recognised how EGTM positions the researcher as the author who reconstructs meaning through the research process, acknowledging that the researcher and the researched co-create the theory.

A justification for why EGTM was used in this study was presented, which acknowledged that whilst there was much written on SH based on other professional groups, scant attention had been paid to the interaction of SH patients with paramedics. As paramedics caring for people who $\mathrm{SH}$ is a phenomenon occurring in the social world, it was discussed how the study harnessed the ability of EGTM to develop explanations of key social processes or structures derived from, or grounded in, empirical data. It was therefore argued that EGTM would facilitate in-depth inquiry of the basis of paramedics' perceptions of caring for people who $\mathrm{SH}$, what 
these perceptions were and why they were formed and could generate explanations which may influence future care through education, policy and practice.

The influence of research philosophy on this study was considered, which included important assumptions about how the researcher observed or viewed the social world. Epistemology was recognised to be a branch of philosophy that studies the nature of knowledge, what constitutes acceptable knowledge in the field of study (Saunders et al 2007), and that is concerned with the nature, validity and limits of inquiry (Rosenau 1992). It was important to recognise the epistemological position within this study as it related to the methods through which knowledge would be acquired. It was discussed how EGTM follows the interpretivist-constructivist epistemological position which sees the world as constructed, interpreted, and experienced by people in their interactions with each other and with wider social systems (Lincoln \& Guba 2000), where meaning is embedded in the participants' experiences and is mediated through the researcher (Merriman 1998). This constructivist paradigm of inquiry accepts that the researcher is a "passionate participant as facilitator of multi-voice reconstruction" (Lincoln \& Guba 2005 p. 196). Other key features of EGTM presented were the notion of theoretical sensitivity, the constant comparative method of data collection and analysis and theoretical saturation.

Theoretical sensitivity was recognised to be a multidimensional concept that includes the researcher's level of insight into the research area, how attuned they are to the nuances and complexity of the participant's words and actions, their ability to reconstruct meaning from data generated with the participant, and a capacity to "separate the pertinent from that which isn't" (Strauss \& Corbin, 1990, p. 44). Theoretical sensitivity was argued to have been cultivated in this study via literature reviews which included a systematic review of the quantitative literature and metasynthesis of qualitative literature. Additionally, the previous experience of the researcher as a paramedic allowed for insight and awareness of the subtleties of the data and the ability to bring meaning to the data (Strauss and Corbin, 1990). 
Debates over the role of the literature review in EGTM were acknowledged within this chapter. It discussed how views differ on the timing of the literature review and how some suggest that prior in-depth study of the literature may violate the emergent nature of qualitative research with pre-conceived ideas. However, it was acknowledged that within EGTM of Strauss and Corbin (1994) researchers should reflect critically on themselves and their behaviour, as well as continuing to positively emphasise the researcher's familiarity with the literature associated with the research area in such a way that it might be played against systematically gathered data. The use of self and the literature were therefore presented as early influences within the study because, while diffuse understandings provided sensitivity, both the literature and specific understandings from past experience were used to stimulate theoretical sensitivity and generate the EGTM.

It recognised that EGTM is a way of generating new theory, grounded in the field but also set in the context of existing theory (McGhee 2007), and it was acknowledged that Strauss \& Corbin (1990) advocate reviewing the literature early in a study as it provides a secondary source of data, stimulates questions, develops stimulates theoretical sensitivity and directs theoretical sampling. The influence of research philosophy in the literature review in this study was argued to include important assumptions about how the researcher observes or views the social world. There was an acknowledgement that areas of literature would contain measurable facts and present established evidence, however it was also accepted that this would be an incomplete exploration of knowledge and literature in this area due to the complexity and multiple influences on paramedics' perceptions of care for those who SH.

Within this chapter, it was recognised that paramedic practice had traditionally lacked an evidence base due to its over reliance on hospital-based practices and those of other professional groups. It was therefore anticipated that there would be few published papers specifically relating to paramedic care for those who $\mathrm{SH}$. As a result the literature review fulfilled several functions: it was exploratory, a method of mapping out areas of uncertainty, identifying whether the proposed study had already been conducted, and where it sat in the body of research. The literature review was also presented to be an attempt to develop theoretical insights within the 
constructivist approach to the study. To meet such broad aims, a rigorous approach was employed which recognised established knowledge in the care for those who SH gained through both quantitative and qualitative methods. This was achieved by a systematic review of the quantitative literature and metasynthesis of the qualitative literature; these are both discussed in more detail in the following chapters.

This chapter recognised that in qualitative research researchers should avoid influencing data with pre-conceived ideas which may inhibit the process of discovery (Jasper 1994, Holloway 2005). To counteract this, a biosketch was presented which considered the researcher's past assumptions and experiences in this area and was reflected upon throughout the study. The biosketch also supported the transparency and trustworthiness of the coding whereby a second researcher independently reviewed the coding framework and reflective notes were made by both researchers, which considered theory development and monitored the researchers' influences on this process. In addition, the primary researcher returned to study participants to check their response to the researchers' interpretation of data, using 'memberchecking' techniques.

The methods of the study were discussed in detail, which involved distribution of a poster requesting volunteer paramedics for the study. Following responses to the poster call, potential participants were selected using theoretical sampling methods, based on the characteristics of age, gender, years of paramedic experience, rural or urban setting, and educational development. These characteristics were identified by the systematic review of and metasynthesis conducted by the researcher, both of which are reported in the following chapters of this thesis. Following the poster call, study participants were contacted by email, thanking them for volunteering to be involved in the study.

The interview guide approach used for this study was presented in this chapter. The interview guide consisted of semi-structured questions to cover the broadly defined agenda that arose from the research questions and literature reviews. Categories from the metasynthesis and systematic review were again used to inform the development of the interview guide. It was discussed how two pilot interviews were undertaken, 
with one paramedic working in an urban area and one paramedic based in a rural area. It was acknowledged that this sampling approach could yield rich patterns emerging from geographical variation, as well as capturing the core experiences, disparate issues and central shared aspects that cut across the service variation. The interview guide was amended following the pilot interviews, and data from these interviews were included in the study proper.

Within this chapter it was noted how theoretical sampling was also a fundamental aspect of the EGTM and involved collecting further data in the light of categories that emerged from earlier stages of data analysis. These methods of data collection involved the paramedic researcher conducting one-to-one interviews, audio-recorded and transcribed verbatim. Transcriptions were read line by line, and coded through the three levels of open, axial and selective coding. NVIVO (2015) V10 software was use in this process. Coding required simultaneous collection, coding and analysis of data, asking of the data what is happening and what it represents. The first stage was open coding in which data were compared for similarities, differences and questions regarding emergent phenomena. Then axial coding developed categories, taking concepts and thinking of how they could be subsumed under a higher-level heading. Next, selective coding was an explication of the story line which involved identifying the basic social process (BSP) at work, around which all other categories revolved. Finally, theoretical constructions conceptualised the relationship among the three levels of coding, by weaving the fractured data back together again to form an evolved grounded theory. Theoretical sampling was further described, where participants were invited for interview based on their characteristics reflecting areas of enquiry being constructed, and data emerged during coding and analysis of interviews; the researcher selected participants who it was felt would yield rich insights into the areas of interest emerging in interviews. Interviews continued until no new information was forthcoming and thematic categories were saturated, where a state of saturation was deemed to have been achieved. 


\section{Chapter Four - Perceptions of emergency care staff of those who Self Harm: A systematic review of the quantitative literature}

\section{Introduction}

It has previously been recognised that the U.K. has one of the highest rates of SH in Europe and that paramedics and emergency staff may be the first professionals to encounter such incidents, therefore understanding their views and approaches to care is crucial. This chapter presents a systematic review of the quantitative literature, a version of which has been published in the Journal of Psychosomatic Disorders (Rees et al 2014) (Appendix I).

Whilst the systematic review sought to include paramedics, no studies were retrieved which included this professional group. It was however important in identifying the lack of published evidence relating to paramedic care for people who SH. The systematic review did include data from other emergency care personnel including doctors and nurses working in an emergency care setting; this provided context to the research, stimulating further questions, theoretical sensitivity and informed development of the interview guide. The systematic review also allowed for reflections and comparisons with the results of the present study as they emerged.

The influence of research philosophy has been acknowledged previously within this thesis. It discussed how epistemology is a branch of such philosophy that relates to how we come to acquire knowledge and the beliefs on the way to generate, understand and use knowledge that are deemed to be acceptable and valid (Wahyuni 2012). The two broad epistemological positions of positivism and interpretivismconstructivism have been previously highlighted, where the interpretivistconstructivist perspective sees the world as constructed, interpreted and experienced by people in their interactions with each other and with wider social systems (Lincoln \& Guba 2000). Positivism however assumes there are social factors with an objective reality apart from the beliefs of individuals (Bahari 2010), and provides evidence in a quantitative form (Sarantakos 2005). Qualitative methodologies assume that meaning is embedded in the participants' experiences and that this 
meaning is mediated through the researcher (Merriman 1998). Adopting a constructivist qualitative approach in this study recognises that it is the researcher whom aims to understand paramedics' perceptions of care for people who SH from the perspective of those experiencing, i.e paramedics. It was therefore the researcher who was the one to construct an account based on the collection of data and interpretations made, and this construction will be influenced by both quantitative and qualitative literature.

The researcher's early reflections recognised that areas of literature would contain objective and measurable facts, and that whilst there was expected to be limited literature on paramedic care for people who $\mathrm{SH}$, it was anticipated there would be an established evidence base gained from such positivist sources from the perspective of other emergency care staff such as doctors and nurses. It was therefore decided to conduct an initial systematic review of the literature of paramedics' perceptions of care for people who SH and, given the potential absence of literature on paramedics, the systematic review also included other emergency care workers such as emergency doctors and nurses. It was recognised however that this would be an incomplete exploration of the literature in this area and so it was decided to conduct a metasynthesis of the qualitative literature of perceptions of paramedics and emergency care staff about the care they provide to people who $\mathrm{SH}$, which is presented later in this thesis.

The following chapter presents the systematic review of quantitative literature of perceptions of paramedic and emergency care workers of those who SH. Emergency care workers are defined as doctors or nurses working in an emergency care setting. The aim of this systematic review was to systematically review published quantitative literature relating to paramedic and emergency care staff perceptions and experiences of caring for people who SH. This systematic review was vital in illuminating established knowledge in this area, and informing the evolving theory on paramedics' perceptions of care for people who SH. 


\section{Methods}

\section{Search strategy}

Electronic literature searches were conducted (June 2013) of CINAHL®, MEDLINE®, OVID $®$ and Psych INFO®, using a broad search strategy with the key words Self Harm, Paramedic and Perceptions. MeSH terms and truncation were applied as follows: [Self Harm* OR suicide*OR overdose*] AND [paramedic* OR nurse* OR doctor*] AND [emergency* OR pre hospital* OR ambulance*] AND [perceptions of care*]. No language or date restrictions were applied. References of included papers and grey literature such as guidelines, policy documents and conference proceedings were also hand searched.

\section{Eligibility criteria}

The Preferred Reporting Items for Systematic Reviews and Meta-Analyses (PRISMA) guidelines (Moher et al 2009) were adhered to (Fig. 5 overleaf). Papers were reviewed independently by two researchers screening their title, abstract and the full papers against the inclusion criteria (Fig 6).

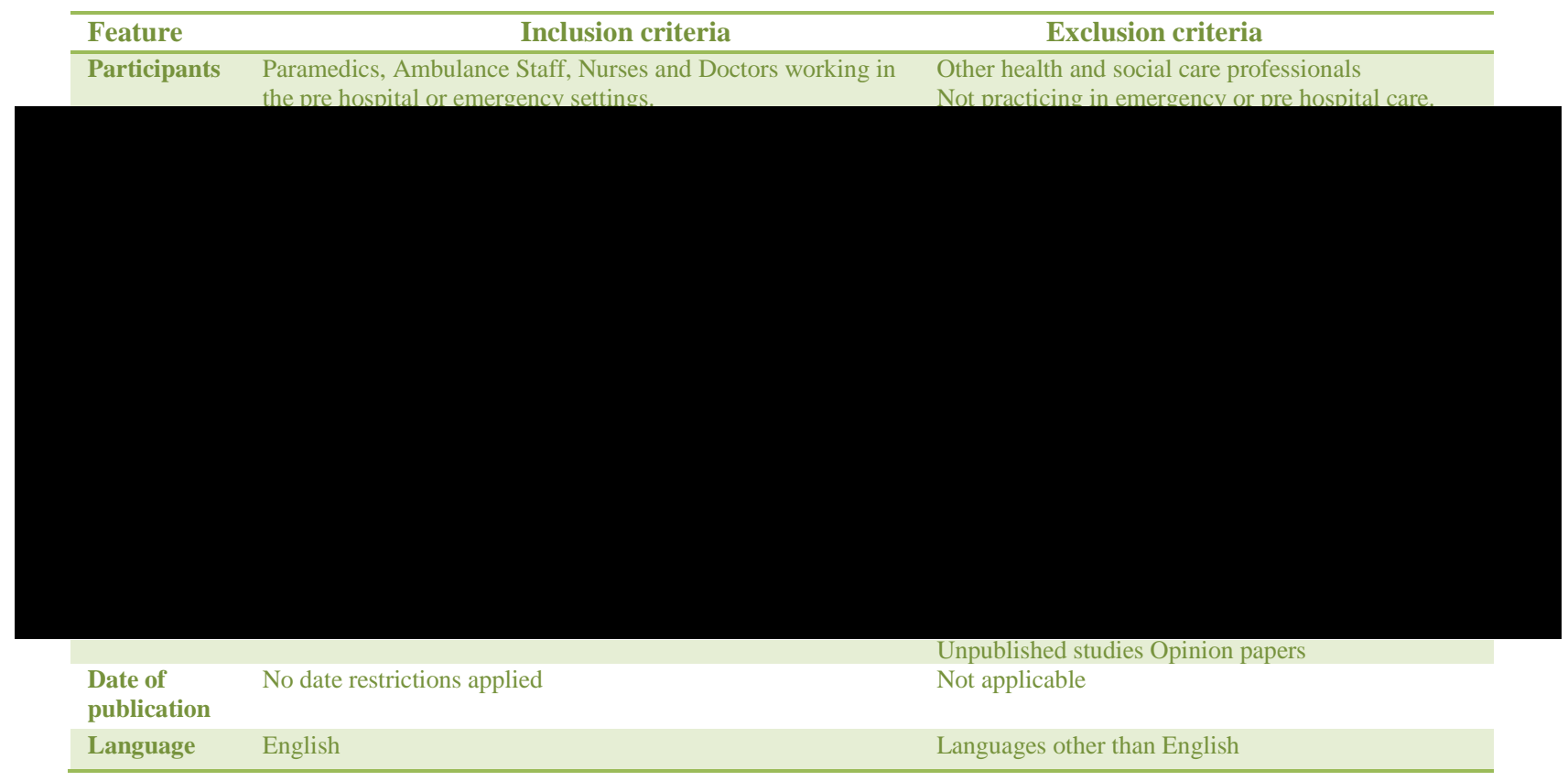

Fig. 6 Inclusion and exclusion criteria 


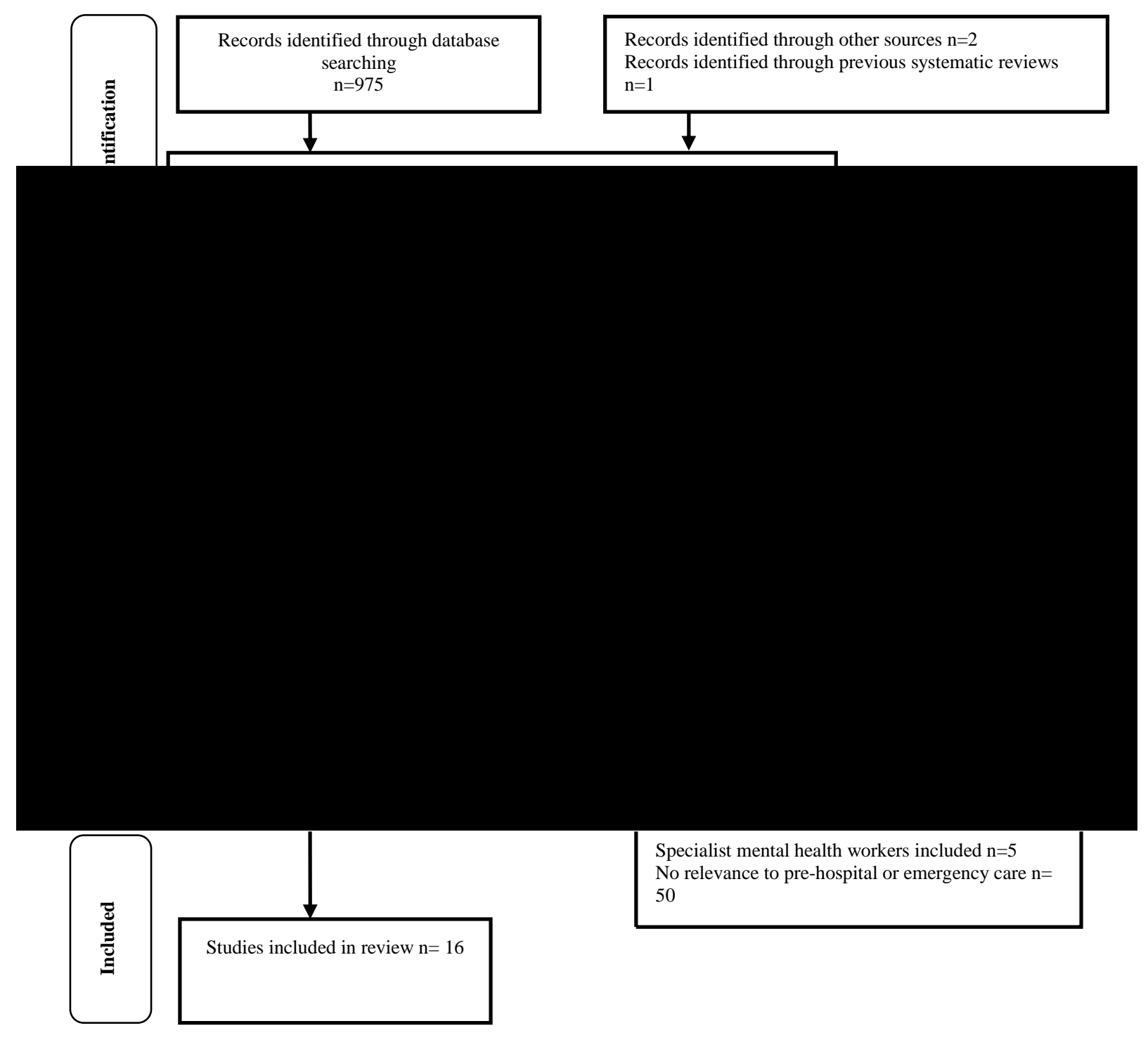

Fig 5. PRISMA Flow Chart 


\section{Quality Assessment}

Quality was assessed using an adapted version of the Social Care Institute for Excellence Quality Assessment Tool (Social Care Institute for Excellence 2006) and the Critical Appraisal Skills Program (CASP 2002). Two reviewers independently assessed the quality of papers and disagreements were discussed at consensus meetings, until the two agreed the final quality of all papers.

\section{Data extraction and synthesis}

Study characteristics and outcomes were recorded on a pre-designed data abstraction form (Figure 7). All studies meeting the inclusion criteria were included in the review regardless of quality however, in interpretation, more weight was given to studies of more robust design and relevance. The number of reports was so limited, and differences between study design and measures so great, that combining results with statistical testing for bias and heterogeneity was inappropriate and meta-analysis was not undertaken. Rather, data synthesis comprised data extraction and descriptive analysis.

\section{Results}

Eight hundred and sixty-four studies were screened for relevance. A random sample was independently reviewed by two reviewers with reasonable agreement at abstract stage (Kappa 0.649 sig. $=0.00)$ and full paper $($ Kappa 0.783 sig. =.011); disagreements were resolved by discussion until the final sample was agreed. Data were abstracted from 16 studies. Participants included emergency doctors, nurses and ambulance staff. One study (Ghodse 1978) included emergency ambulance staff but did not include paramedics as the study was conducted before the introduction of paramedics in the UK. All studies used questionnaires and scored an average of 9 out of 12 on quality assessment; this was comparable to a previous literature review conducted by Saunders et al (2009). Studies were conducted in the UK $(n=5)$, Australia ( $n=4)$, Ireland ( $n=4)$, Taiwan $(n=1)$, Helsinki $(n=2)$. 
Fig. 7 Systematic review data abstract

\begin{tabular}{|c|c|c|c|c|c|c|}
\hline Study & Quality & $\begin{array}{l}\text { Number of } \\
\text { subjects }\end{array}$ & Clinician & Setting & Methods & Outcomes \\
\hline $\begin{array}{l}\text { Friedman } \\
\text { et al } \\
(2006)\end{array}$ & 8 & 117 & $\begin{array}{l}\text { ED staff } \\
88 \text { nurses } \\
29 \text { doctors. }\end{array}$ & E/D UK & Questionnaire & $\begin{array}{l}\text { Staff believed that self-laceration was an important problem } \\
\text { relationship between self-laceration and both mental illness a } \\
\text { in SH. In those without previous training, a longer period wo }\end{array}$ \\
\hline
\end{tabular}

29 doctors in SH. In those without previous training. a longer period wo
in

\begin{tabular}{|c|c|c|c|c|c|c|}
\hline $\begin{array}{l}\text { McAllister } \\
\text { et al } \\
(2008)\end{array}$ & 9 & 27 & $\begin{array}{l}\text { Emergency } \\
\text { nurses }\end{array}$ & $\begin{array}{l}\text { Queensland } \\
\text { Australia }\end{array}$ & $\begin{array}{l}\text { Pre-test/post-test } \\
\text { design }\end{array}$ & $\begin{array}{l}\text { No significant improvement in the global measure of nurses' } \\
\text { Significant improvements }(\mathrm{p}<0.05) \text { from pre- to post-test fo }\end{array}$ \\
\hline $\begin{array}{l}\text { McAllister } \\
\text { et al } \\
\text { (2002a) }\end{array}$ & 9 & 1008 & $\begin{array}{l}\text { Emergency } \\
\text { nurses }\end{array}$ & $\begin{array}{l}23 \text { public } \\
\text { and } 14 \\
\text { private ED }\end{array}$ & $\begin{array}{l}\text { The Attitudes } \\
\text { Towards } \\
\text { Deliberate Self- }\end{array}$ & $\begin{array}{l}\text { Four factors reflected nurses' attitudes toward SH: } \\
\text { - } \quad \text { Perceived confidence in their assessment and referral sh } \\
\text { - } \quad \text { Ability to deal effectively with clients }\end{array}$ \\
\hline
\end{tabular}




\section{Queensland}

Australia

Questionnaire

(ADSHQ)

Mc Alister 10

(2002)
1008

(1)

Nurses

es

ED

Queensland

Australia
- Empathic approach

- $\quad$ Ability to cope effectively with legal and hospital regu $90 \%$ had no formal training in managing clients who SH

Staff in large hospitals scored less empathy towards SH clien Significant correlations were found between years of ED exp experience and an empathic approach towards $\mathrm{SH}(\mathrm{p}<0.01)$ Generally negative attitude towards clients who SH.

Correlations between ED experience and ADSHQ score, yea

$96.3 \%$ indicated professional experience with people who S

$36.1 \%$ indicated personal experience with people who SH.

\section{Suokas \& \\ Lonnqvist}

(1989)

184

84

(1)

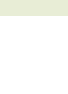

\begin{abstract}
Nurses \&
Doctors
\end{abstract}

ED

comprising

of

Emergency

room (ER),

Emergency

ward (EW) $90.1 \%$ had not had any formal training in $\mathrm{SH}$

Questionnaires 

suicide $(\mathrm{p}<0.001)$

$76 \%$ of ER, $40 \%$ EW and $23 \%$ of ITU staff agree that a pati

$28 \%$ of ER staff aoreed completely that patients who attemp

LaugnIIII
(1994)

Who tried to Kill themselves ald not really want to die ( $P<$ U attempt $(\mathrm{P}<0$ 05), disagreed that those who attempt suicide u attention $(\mathrm{P}<005)$

More experienced nurses than less experienced nurses agree from others $(\mathrm{P}<001)$ 


\section{Critique of quality of the literature included in the systematic review:}

The quality of the literature included in the systematic review was assessed using an adapted version of the Social Care Institute for Excellence Quality Assessment Tool (Social Care Institute for Excellence 2006) and the Critical Appraisal Skills Program (CASP 2002). This approach was previously used in systematic reviews of care for people who SH (Taylor et al 2009, Saunders et al 2012). Studies were scored based on the number of positive answers to the questions posed in these tools. As these were all quantitative studies, they were given a further two points (as in Saunders et al 2012), to counter the two additional questions that were only applicable to qualitative studies. Two review authors independently assessed the quality of studies and disagreements were then discussed at consensus meetings and final quality score agreed.

The quality of studies was generally reasonably high, with a mean score of 7.5 out of a maximum of 12. This was comparable to Taylor et al (2009) and Saunders et al (2012) who used the same quality appraisal method with the average score of studies being 8.02 out of a maximum of 12 . All the studies used questionnaires, which may be considered a low-quality research method and somewhat limited, as the results may only reflect the conscious feelings of the respondent. However, due to the nature of the research question focusing on perceptions of emergency care staff of those who $\mathrm{SH}$, these methods were appropriate for this subject area. Questionnaire studies are useful as baseline measures in intervention studies to assess the effectiveness of projects targeting attitudinal changes (McAllister et al 2002). This does however also emphasise the need for qualitative designed studies which may also illuminate the findings in further depth.

The higher quality studies included in the review (Mc Laughlin 1994, Mc Alister 2002, Egan et al 2012, Conlon \& O'Tuathail 2012, McCarthy \& Gijbels 2010, Sun et al 2011) met much of the quality assessment criteria by including features such as a clearly focused research question, well defined participant selection and a clearly justified recruitment strategy and research methods. The methods within the higher scoring studies also all included validated questioners such as those discussed earlier, to include the Self-Harm Antipathy Scale (Conlon \& O'Tuathail 2010), Attitudes towards Deliberate Self-Harm Questionnaire (McCarthy et al 2010), Suicide Opinion Questionnaire (Sun et al 2007, McCann et al 2006), or Understanding Suicidal Patients 
Scale (Suokas et al 2009). As the systematic review focussed on $\mathrm{SH}$, it has already been emphasised in this thesis that there are a multitude of definitions in the literature for $\mathrm{SH}$. Whilst no studies used the NICE (2014) definition for SH, higher quality studies made some attempt to define $\mathrm{SH}$.

Many of the studies were conducted in one hospital, utilized a convenience sample of ED nurses, and had a low number of participants, which ranged from 27 to 1008. These factors thereby limited the ability to generalize the findings to other settings. Few of the studies also reported how they derived at their sample size. Sample size is a key feature in the quality of a study design, as it can influence the detection of significant differences, relationships or interactions (Peers 1996). In surveys designs one attempts to minimize both alpha error (finding a difference that does not actually exist in the population) and beta error (failing to find a difference that exists in the population) (Peers, 1996). A common goal of survey research is therefore to collect data representative of a population. Wunsch (1986) argues that two of the most consistent flaws in survey designs disregard for sampling error when determining sample size, and disregard for response and nonresponse bias. Studies in the present systematic review with well justified sample selection and recruitment, which yielded a high response, were therefore rated higher in the study.

In studies such as Suokas, Suominen, Lonnqvist (2009) and Mackay \& Barrowlough (2005), less than half of the questionnaires were returned in the second stage of the study. This was explained by the busy and unpredictable workload of emergency room staff. However, these studies also had no information on those who failed to return the questionnaire, and so no comparison could be made with those who returned and those who did not. The studies samples may therefore have been biased. This may be of significance and could limit the validity of the inferences made and conclusions from such findings. For instance, in Suokas, Suominen, Lonnqvist (2009), after a year of a consultation service being introduced into ED for people who attempted suicide, they found that understanding and willingness of ED staff to care for people who attempted suicide had not significantly changed. However, the details of the non-responders are not known, and the low response rates during the second stage of the study occurred when the emergency room had long been understaffed. Therefore, issues such as 
business and increased workload could be influencing increased negativity in staff, thus counteracting any potential benefit the consultation service may have brought. This may be an example of such a beta error discussed earlier, where a researcher may have failed to find a difference that existed in the population (Peers 1996). Studies which acknowledged such limitations were scored higher.

\section{Outcome variables, instruments and data analysis}

Participant demographic and descriptors varied considerably and included attitudes to $\mathrm{SH}$, age, gender, experience, and previous SH training. There was also variation in measurement instruments; most studies used structured questionnaires, often selfdeveloped, reflecting the lack of reliable and validated measures specifically targeting self-harm. The validity and reliability of these instruments was not always described. Six studies used validated measurement instruments such as the Self-Harm Antipathy Scale (Conlon \& O'Tuathail 2010), Attitudes towards Deliberate Self-Harm Questionnaire (McCarthy et al 2010), Suicide Opinion Questionnaire (Sun et al 2007, McCann et al 2006), or Understanding Suicidal Patients Scale (Suokas et al 2009). As the present systematic review included only quantitative studies, a range of descriptive or inferential statistics were used in the data analysis.

The following themes were identified: SH training and education, Policies for dealing with people who SH, Attitudes towards people who SH, Factors influencing attitudes towards people who SH: age and experience, gender and setting.

\section{SH training and education}

Many studies reported a lack of training for health professionals caring for people who $\mathrm{SH}$; previous training was reported by $18 \%$ to $22 \%$ of staff (McCarthy et al 2010 , McAllister et al 2008), 75\% to 90\% had not received any training (McAllister et al 2008, Egan 2012, McLaughlin 1994). Staff believed they lacked the necessary skills to care for people who $\mathrm{SH}$, and perceived the need for further training in communication and understanding of $\mathrm{SH}$, which they believed would give them more confidence (Friedman et al 2006, Conlon \& O'Tuathail 2010, Sun et al 2007). When such programmes were attended, significant improvements were reported in knowledge, confidence and attitudes towards SH (Crawford 1998, McCarthy et al 2010, Friedman 2006, McCann et al 2006, Turnbull \& Chalder 1997). Three studies reported on the 
impact of education on professional development. Attitudes to $\mathrm{SH}$ were more positive in nurses prepared through diplomas than hospital trained nurses (McCarthy et al 2010, Sun et al 2007), and medical staff indicated less of a perceived need for further training about the impact of SH on patients than nurses $(\mathrm{p}<0.0001)$. (Macka \& Barrowclough 2005, Friedman et al 2006) administered a questionnaire to 117 Emergency Department (ED) staff and the results indicated that whilst they felt self-laceration was an important problem, they felt unskilled in dealing with these patients. Knowledge and confidence were significant contributors to perceived personal effectiveness in dealing with people who SH (Egan et al 2012). A positive relationship was found by Egan et al (2012) between knowledge and effectiveness $(\mathrm{p}<0.05)$; and between confidence and effectiveness $(\mathrm{p}<0.01)$ and an opposite (negative) relationship was found between effectiveness and negativity $(\mathrm{p}<0.01)$.

\section{Policies for dealing with people who SH}

Two studies reported that policies or guidelines for treating SH were lacking or underused. In McCann et al (2006) 20\% of nurses claimed the ED either had no practice guidelines for $\mathrm{SH}$ or, if they did, that nurses were unaware of their existence, though a large minority (one-third) knew about them but had not read them. $46 \%$ of respondents in McAlister (2002 b) indicated neither formal nor informal procedures existed for managing those who $\mathrm{SH}$ and $82 \%$ reported having no guidelines in place for assessing SH. McCarthy \& Gijbels, McCarthy et al (2010) also reported that 68\% of ED staff agreed that hospital policies impeded their ability to work effectively with $\mathrm{SH}$ patients.

\section{Attitudes towards people who SH}

Several studies reported positive attitudes towards people who SH (McCarthy et al 2010, McLaughlin 1994, Conlon \& O’Tuathail 2010, McCann et al 2006, Suokas \& Lonnqvist 2009). Sun et al (2007) revealed how nurses displayed empathy towards those who SH, perceiving suicide attempts as people trying to communicate their pain and being keen to seek attention.

Evidence was found to indicate that some staff perceive $\mathrm{SH}$ as deserving a lower priority of care than conditions they perceive as purely 'medical' (McLaughlin 1994, Suokas \& Lonnqvist 1989, Ghodse 1978). Mc Laughlin (1994) reported in response to a 
hypothetical scenario that all respondents felt a patient with severe chest pain required urgent nursing intervention, whilst $18 \%$ of younger nurses and $11 \%$ of older nurses felt that the overdose patient did not. Suokas \& Lonnqvist (1989) reported that $76 \%$ of Emergency Room (ER) staff agree that patients who attempted suicide wasted staff time, $28 \%$ of staff agreed these patients misuse treatment facilities, and $51 \%$ of staff agreed that these patients take up staff time that is sorely needed by others in greater need of help. In Ghodse (1978), ambulance staff had significantly more favourable attitudes to accidental overdoses than any other professional group whereas they expressed an unfavourable attitude towards drug-dependant patients.

The impact of SH encounters on risk of future $\mathrm{SH}$ or completed suicide was raised in several studies (Suokas \& Lonnqvist et al 1989, McCann et al 2006, Sun et al 2007, McLaughlin 1994). 30\% of emergency room staff in Suokas \& Lonnqvist (1989) disagreed that attitudes and behaviour of staff influenced recurrence of suicide attempts.

Mc Alister (2002 a) found that $96 \%$ of respondents in a study of seventy-one nurses working in two EDs in Australia reported professional experience of caring for people who $\mathrm{SH}$, whilst $36 \%$ reported personal experience of $\mathrm{SH}$ with friends or family. Freidman et al (2006) however found that incidence of SH was overestimated by UK ED staff; the mean estimate of SH presentations to one ED in a six month period was 117 cases, while the actual number was 22 cases.

\section{Factors influencing attitudes towards people who SH}

In studies reporting negative attitudes, the influencing factors presented a complex picture. McAllister et al (2002a.) found an Attitudes towards Deliberate Self-Harm Questionnaire (ADSHQ) score of 65.16 ( $\mathrm{SD}=4.38$, range=46-87), indicating generally negative attitude towards patients who SH in ED nurses. However, ADSHQ identified four dimensions explaining variations in attitudes, which included perceived confidence in assessment and referral, ability to deal with clients, legal and hospital regulations guiding practice, and an empathic approach. Amongst staff who had not been trained in $\mathrm{SH}$ a longer period of ED working was correlated with higher levels of anger toward patients who SH. Nurses reported in Conlon \& O'Tuathail (2010) that patients who repeatedly presented following $\mathrm{SH}$ evoked feelings of frustration and powerlessness and 
were often ignored or marginalised. Sun et al (2009) found ED nurses perceived suicidal behaviour as irritating, and in McAllister et al (2002a.) respondents were more likely to agree that they felt helpless in dealing with people who SH.

The effects of caring for people who SH on professional attitudes were mixed, with several studies showing a positive relationship between level of experience of caring for people who SH and unfavourable attitudes (Ghodse 1978, Sun et al 2007, Friedman 2006). McAllister et al (2002 a.) administered the 'Attitudes towards Deliberate SelfHarm Questionnaire (ADSHQ) to nurses working in EDs in Australia. The possible total scores for attitudes in ADSHQ range from 25 to 100; scores ranging from 25 to 62 are considered negative, while scores ranging from 63 to 100 are considered positive. McAllister et al (2002 a.) found significant correlations $(\mathrm{p}<0.05)$ between years of professional caring experience and ADSHQ total score, and years of experience.

Older caregivers had more empathy and understanding for patients who SH. McCann et al (2006) found that older and more experienced nurses had more supportive attitudes than younger and less experienced nurses $(\mathrm{p}<0.04)$; older staff also displayed greater empathy than younger staff and held more positive attitudes towards people who SH (p $<0.05$ ) (Conlon \& O’Tuathail 2010). Mc Laughlin (1994) administered an adapted Suicide Opinion Questionnaire (SOQ) to 200 nurses working in EDs, and found that more older nurses than younger nurses believed that those who tried to kill themselves did not really want to die $(\mathrm{P}<0.05)$, those who threatened to commit suicide rarely did so $(\mathrm{p}<0.05)$, and those who attempted to commit suicide often failed ( $\mathrm{p}<0$ 05), but disagreed that those who attempted suicide using highly public places are more interested in getting attention ( $p<0$ 05). In the study by McCann et al (2006) younger nurses were undecided in their response to the statement that individuals who attempted suicide were trying to get sympathy from others, whereas older nurses were more likely to disagree $(\mathrm{p}<0.01)$

Mc Cann et al (2006) reported that experienced nurses were more likely to disagree that individuals who threatened suicide rarely took their own lives $(\mathrm{p}<0.01)$, whilst in $\mathrm{Mc}$ Laughlin (1994) 71\% of less experienced nurses and $92 \%$ of more experienced nurses agreed that most people who tried to kill themselves did not really want to die $(\mathrm{P}<0$ 
$001), 53 \%$ of younger nurses and $65 \%$ of older nurses tended to agree that most people who attempted suicide failed in their attempt $(\mathrm{P}<005)$.

Religious beliefs may also be a factor in attitudes to SH care. In Sun et al (2009) almost $50 \%$ revealed they held religious beliefs; nurses with no religion had more positive attitudes towards suicidal behaviour than those who followed a religion.

Staff in acute settings and large units reported higher levels of negativity towards people who SH than those in non-acute settings (Suokas \& Lonnqvist 1989). Suokas \& Lonnqvist (1989) found $72 \%$ of staff in the Intensive Therapy Unit (ITU), 58\% in the Emergency Ward (EW) and 25\% in the Emergency Room (ER) claimed they were as co-operative and sympathetic towards patients who attempt suicide as other patients ( $p<0.001)$. Sixty per cent of EW, 54\% ER, 16\% of ITU staff admitted to letting their irritation show when treating people who SH (p<0.001). McAllister et al (2002a.) also found staff in large hospitals scored significantly lower on perceived ability to assess and refer SH clients $(\mathrm{P}<0.05)$ and were less empathetic towards $\mathrm{SH}(\mathrm{p}<0.05)$.

The proportion of female respondents ranged from $84 \%$ to $95 \%$ across studies. Two studies found associations between gender and attitude towards SH: Ghodse (1978) found significantly more males expressed unfavourable attitudes towards drug dependence and suicide overdose patients $(\mathrm{p}<0.01)$. Mackay \& Barrowclough $(2005)$ reported male staff expressed less sympathy $(\mathrm{p}<0.02)$, greater irritation $(\mathrm{p}<0.04)$ increased frustration $(\mathrm{p}<0.01)$, and a lack of willingness to help $(\mathrm{p}<0.005) \mathrm{SH}$ than their female colleagues. The picture was mixed however as two studies failed to demonstrate any significant gender related findings (Suokas et al 2009, McCarthy et al 2010).

\section{Discussion}

All studies reviewed used questionnaires and scored an average of 9 out of 12 on quality assessment with the Social Care Institute for Excellence Quality Assessment Tool (Social Care Institute for Excellence 2006), and whilst this is comparable to previous studies (Saunders et al 2012) such self-reported data does not provide an indication of what is actually happening in practice. 
This review identified one study of the care provided by ambulance staff for patients who SH (Ghodse 1978). That study is forty years old and was undertaken before the introduction of the professionalisation of paramedics in the UK as they were not accepted to register with the Health Professions Council until 2001. As paramedics are often the first professionals encountered by patients who $\mathrm{SH}$, understanding their views and approaches to caring for people who $\mathrm{SH}$ is crucial as it may determine the quality of care these patients receive. Staff in acute settings were found to exhibit higher levels of negativity towards $\mathrm{SH}$, and attitudes became less positive the closer to the frontline they worked (Suokas \& Lonnqvist 1989). This was linked to situational factors such as competing priorities in a busy ED, and patients being more difficult to deal with earlier in their care. Such findings may not directly transfer to the paramedic population because, whilst they may be at the front line and encountered early in the care of patients who SH, with a comparable workload to emergency staff, they usually have fewer competing priorities, caring for one patient at a time. A recent report by the All Party Parliamentary Group on Mental Health (APPG 2015) gives some insight into the potential for differing perceptions between emergency staff and paramedics. They found some people who SH felt they were not treated with respect or listened to and were stigmatised in the ED, whilst the same report included ambulance staff in their accounts of professionals who had supported them in a crisis being compassionate, supportive and understanding.

Guidelines clearly state that training is needed for paramedics and other emergency care professionals when caring for people who SH (RCPsych 2010, NICE 2004). This systematic review has revealed scant evidence of the availability or uptake of such training. The finding of several papers in this review shows that attendance at such training improves staff knowledge and confidence and lowers negative attitudes towards those who SH. The positive impact of education on attitudes (McCarthy \& Gijbels 2010, Sun et al 2007, Macka \& Barrowclough 2005) may merit further expansion and this should be clearly written into policy documents. Guidelines NICE (2004) also recommend that care pathways, protocols and local guidelines are reviewed and revised. Despite this, studies report a lack of adoption or knowledge of guidelines, (McCann et al 2006, McAllister et al 2002, McCarthy et al 2010) representing a limited influence of such guidelines. The importance of this finding links with earlier assertions that the 
awareness and availability of guidelines and policies will support the positive relationships between knowledge, confidence, perceived personal effectiveness and decreasing negativity. Addressing the educational needs and availability of guidance for staff has the potential to positively impact on care for people who SH when they encounter paramedic and emergency care staff and is therefore a key recommendation of this study.

Staff with greater experience of care held more negative attitudes and more anger towards patients who SH (Ghodse 1978, Sun et al 2007), however results were inconsistent, as studies reported a significant relationship between the duration of clinical experience and attitudes to SH (McAllister et al 2002), and significant correlations between years of ED experience and an empathic approach towards SH patients (McLaughlin 1994). It is therefore unclear whether experience influences staff attitudes. Recent studies reported more positive attitudes from emergency staff which contrasts with a recent systematic review (Saunders et al 2012) and studies investigating the patients' perspective (Warm, Murray \& Fox 2002, Cleaver 2013, Taylor 2009, NCCMH 2004). A recent scoping review of attitudes of emergency care staff towards young people who SH (Cleaver 2013) recognised benign attitudes towards $\mathrm{SH}$ in recently published studies which reflects the mixed picture of our findings.

The findings of more positive attitudes towards SH from emergency staff is unclear; it may demonstrate the influence of education, policy, guidelines or media reporting, but this has yet to be examined. Studies finding negative attitudes presented a more complex picture than simply positive or negative factors such as perceived confidence in assessment and referral, ability to deal effectively with clients, empathic approach, and ability to cope effectively with legal and hospital regulations that guide practice, may be influencing this complex picture (McAllister et al 2002, Egan et al 2012). Staff who scored higher on these factors were more likely to feel positive toward people who SH and the care they provide. SH evokes feelings of irritation and anger (Conlon \& O’Tuathail 2010, McAllister et al 2008, Sun et al 2007, Suokas \& Lonnqvist 1989, Friedman et al 2006) borne out of a sense of powerlessness and frustration with patients who SH frequently returning to hospital. These perceptions appear to manifest themselves by $\mathrm{SH}$ patients being given a lower priority than those with a physical 
illness, and reduced entitlement to care. Cleaver (2013) argues that staff downgrade the psychological dimensions of care when under pressure, prioritising physical care which is less time consuming and resource intensive, particularly in the ED.

The established link between SH and completed suicides was investigated by several studies in the review (Sun et al 2007, Suokas \& Lonnqvist 1989, McCann et al 2006, McLaughlin 1994, Crawford 1998). Emergency staff acknowledged the risk of completed suicides in people who SH, which is called for in policy (NICE 2000), RCPsych (2004), however more experienced staff showed more awareness of this risk. One study found a quarter of suicides to be preceded by acts of SH within the previous year (Owens et al 2002), therefore this awareness of experienced staff may be borne from previous encounters with such patients who SH and subsequently kill themselves. Such findings may be used to inform education, targeting inexperienced staff and highlighting the risk of suicide in people who $\mathrm{SH}$.

Belief in a religion has been identified as a protective factor against $\mathrm{SH}$ and suicide (Faruqui \& Afghan 2011, Miller et al 2012, Rasic et al 2009, Oliffea et al 2012) and this study found acknowledgement of this amongst staff. However, Sun et al (2009) found a relationship between religion and negative attitudes, $42 \%$ of participants in Sun et al (2009) were Buddhists, where life is believed to comprise of transmigration, and the soul of those who complete suicide cannot be reincarnated (Tzeng \& Lipson 2004). Such a relationship between religion and negative attitudes was also found by Norheim et al (2013), where Christians were significantly less likely to agree that suicide is acceptable than professionals with no religious background. Such a link may be due to historical religious attitudes portraying $\mathrm{SH}$ and suicide as sinful and, whist religion may be influencing attitudes to $\mathrm{SH}$, it is not consistently observed in studies (Ouzouni \& Nakakis 2013).

Females were over-represented in comparison to males in all studies; this is an important factor as two studies found females held significantly more positive attitudes towards SH than males (Macka \& Barrowclough 2005, Ghodse 1978). These studies were dated, and there was a strong gender-role association (male doctors and ambulance staff and female nurses). This may be seen in Ghodse (1978) where females 
had more favourable attitudes to $\mathrm{SH}$, at a time when nursing was predominantly a female profession whereas the opposite was the case for ambulance staff. More recent studies fail to find significant differences in gender (Suokas et al 2009), McCarthy et al 2010), so once again such a mixed picture reveals the complexities of influences on perceptions of care for people who SH.

The insights gained from the systematic review served to further develop theoretical sensitivity within the researcher in the present study, along with stimulating questions, informing the interview guide and sampling, in line with Strauss \& Corbin's (1990) suggestion. The systematic review, however, found only one study relating to ambulance staff, and this was prior to the introduction of paramedics in the UK. Exploring such literature gained from other professional groups and its subsequent transferability to paramedic care was therefore limited. Within the literature, the setting and context in which care for people who SH was explored by these studies was important as these issues had the potential to influence paramedics' and emergency care staff perceptions of caring for those who SH. Only one study in the systematic review considered the pre hospital context. It was therefore acknowledged the systematic review was an incomplete exploration of the knowledge and literature in paramedic care for SH due to the complexity and multiple influences on paramedics' perceptions of care for those who SH.

\section{Limitations}

The authors recognise that as perceptions of SH care involves a phenomenon occurring in the social world, understandings abstracted from quantitative studies will be an incomplete exploration of the literature due to such complexities. The authors are clear to distinguish the positivist position of quantitative literature from the interpretivistconstructivist position of qualitative literature, which is explored through a metasynthesis of the qualitative literature along with an ongoing Evolved Grounded Theory. Studies in the review used surveys, yet such self-reported data may not reflect actual perceptions, moreover evaluating the impact of education may teach the desired answers. The ongoing research considers the patient's perspective along with deeper insights by interviews. The review may also be limited by its search strategy and inclusion of only English language articles. It was considered this sample of staff would give the closest insight into perceptions from staff providing care in the immediate 
aftermath of SH; mental health staff and non-ED staff were excluded due to the potential for specialist knowledge and non-acute setting influencing differing perceptions. In excluding these staff and settings, several high-quality papers were not entered into the study. There also exists potential for publication bias, where there is selective reporting of complete studies with positive results. To limit these potential biases, we hand-searched reference lists and also contacted first authors of all included studies to ask about unpublished material but this had a very low yield. Finally, five out of the sixteen studies retrieved were twenty years old or more.

\section{Conclusion}

People who SH often report that the care they receive from health staff is unsatisfactory and yet paramedics and emergency staff are often the first professionals in contact with people who SH. This study shows that some studies have been done on the attitudes of emergency nurses and doctors but only one has enquired into those of ambulance service staff and none have been carried out into modern professional paramedic care. The initial contact with patients following SH is a vital factor in these patients' acceptance or rejection of care, and may impact future SH behaviours.

This study found the lack of emergency departmental policy on SH was an issue affecting perceived confidence amongst staff and limited formal or informal procedures existed for managing people who SH. This is of concern, as it may represent a failure of national guidelines to influence local and departmental policies aimed to guide paramedic and emergency staff in their care for people who SH. This was also the case in relation to training in caring for people who $\mathrm{SH}$; most staff reported never receiving such training. Again, this is of concern as national policy and guidelines advocate staff receive this training, yet the findings in this study point to a potential failure of such calls to translate into the adoption of training for staff in practice.

Counter to findings from the patient perspective, there is evidence that emergency staff increasingly hold positive attitudes towards patients who SH. However, the picture is more complex than simply positive or negative. Attitudes are multi-dimensional, where SH can evoke feelings of irritation, anger, powerlessness and frustration in staff and, despite calls to receive the same priority and attention as asthma or myocardial infarction, patients who SH appear to be given a lower priority and legitimacy of care 
by staff. The perceptions of care are therefore influenced by educational preparation of staff, experience of caring for $\mathrm{SH}$, age, religious belief, gender, setting, policies for dealing with people who SH and perceived confidence, assessment, referral and ability to cope with legal responsibilities. Finally, it was found that staff in acute settings exhibit increased feelings of negativity, becoming less positive the closer they are to front line care; this was linked to the busy workload in ED and competing priorities. These findings will be of importance to paramedics because, whilst ED staff and paramedics share similarities in workload, few competing priorities present to paramedics who deal with one patient at a time representing an important area which is under investigated. A paramedic may be the first health professional encountered by a patient following $\mathrm{SH}$, and the lack of understanding of this encounter may be concealing the influence and potential contribution paramedics can make in the care of people who SH. This review continues to evolve our understanding of care for people who $\mathrm{SH}$, however further research is urgently required to explore the influence of these themes within the paramedic population. 


\section{Chapter Five - Perceptions of paramedics and emergency care staff about the care they provide to people who self-harm: Constructivist metasynthesis of the qualitative literature}

\section{Introduction}

It has previously been discussed how reviewing of literature within this study was ongoing throughout, and the rationale for conducting a systematic review of the quantitative literature and metasynthesis of the qualitative literature was presented. It was discussed why such an approach was employed, which included the need to recognise the established knowledge in the care for those who $\mathrm{SH}$, gained through both quantitative and qualitative methods. This discussion considered the influence of research philosophy, reflecting on the two broad epistemological positions of positivism and interpretivism-constructivism. Epistemological challenges and tensions were discussed in relation to conducting reviews of the literature derived from qualitative and qualitative forms, especially in EGTM.

The systematic review of the quantitative literature presented in the last chapter explored areas of literature containing measurable facts within the positivist domains of acquiring knowledge, and presents established quantitative evidence in relation to care for people who SH. The researcher's early reflections of the literature and the approach to reviewing such literature recognised such limitations and the epistemological challenges posed in combining insights from established evidence gained from positivist quantitative sources with those from qualitative or interpretive constructivist sources. The reasons for considering the qualitative literature separately from the quantitative literature included an acknowledgment of the strengths of a metasynthesis of qualitative research, where insights are combined from a range of qualitative methodological approaches, offering the potential for a much richer, more flexible, and dynamic approach to evidence and theory development than other traditional systematic reviews of literature (Downe 2008). A metasynthesis was used as it is a structured way to combine learning from multiple qualitative studies, and can also account for complex dynamic processes, allowing the voice of the synthesiser to be heard most clearly (Dixon-Woods et al 2004). This approach therefore complemented the constructivist nature of EGTM being used in the wider study, as the paramedic researcher was a 
"passionate participant as facilitator of multi-voice reconstruction" (Lincoln \& Guba 2005 p. 196) thereby supporting a narrative richness and uniqueness of knowledge produced.

Whilst a rigorous and reproducible approach to retrieving the literature was adopted, the constructed narrative and development of metaphors was facilitated by the paramedic researcher; this is very different to the objective or detached approach to accumulation of logic which occurred within the systematic review of the quantitative literature.

In this chapter, a metasynthesis of the qualitative literature is presented exploring perceptions of paramedics and emergency staff about the care they provide to people who SH. A version of this work has been published in the Journal of Psychosomatic Disorders (Rees et al 2015). (Appendix J). The aim was to enhance knowledge building and theory generation to develop practice and policy through a metasynthesis of qualitative research relating to perceptions of paramedic and emergency care for people who SH.

\section{Methodology}

Whilst there are no universally agreed procedures for synthesising qualitative research, a wide range of methodologies for such systematic inquiry and synthesis of qualitative literature have emerged in the qualitative health research community. In recent years however, qualitative metasynthesis has evolved as a comprehensive generic term representing the family of methodological approaches to developing new knowledge based on rigorous analysis of existing qualitative research findings (Thorn et al 2002). Qualitative metasynthesis is a methodology in which qualitative findings from existing research reports are systematically acquired, qualitatively analyzed and synthesised (Finfgeld 2003, Finfgeld-Connett 2010). Unlike synthesis of quantitative research, qualitative metasynthesis does not involve data aggregation or secondary analysis of raw data, it does not involve accumulation of logic averaging across studies, it is not a type of meta-analysis and nor are the validity of findings in qualitative metasynthesis dependent on their ability to be replicated as occurs in synthesis of quantitative literature (Thorne et al 2002, Finfgeld-Connett 2012). Rather, qualitative metasynthesis results in novel interpretations of qualitative findings that cannot be identified in 
original research reports (Thorne 2002, Noblit \& Hare 1988, Sandelowski 2004). Sandelowski et al (1990 p 366) define qualitative metasynthesis as:

"theories, grand narratives, generalizations, or interpretive translations produced from the integration or comparison of findings from qualitative studies"

When presenting qualitative metasynthesis, validity is based on trustworthiness (Lincoln \& Guba 1985) which can be established through transparent data collection, extraction and analysis methods, clearly reported (Finfgeld-Connett 2010). The qualitative metasynthesis within the present study followed the methodology used by Finfgeld-Connett (2012) that was inspired by the work of Noblit and Hare (1988), Miles and Huberman (1994), and the EGTM approach of Corbin \& Strauss (2008). The procedural guidelines of Noblit and Hare (1998) served as an overarching framework for the present study, and is a methodology widely used for metasynthesis (Attree 2005, Sandelowski \& Barroso 2005, Evans \& FitzGerald 2002, Swartz 2005). Noblit and Hare (1998) suggest that synthesis is achieved by translation, which entails examining key concepts in relation to others in the original study and across studies, and follows the seven steps outlined below. Within these seven steps, detailed synthesis and translation is achieved through the three levels of open, axial and selective coding of (Strauss \& Corbin 1990), which reflects the constructivist nature of EGTM used in the wider study. The framework and coding procedures used in the metasynthesis are presented below:

\section{Getting started (the search)}

A search was undertaken of the databases CINAHL $®$, MEDLINE®, OVID $®$ and Psych INFO®. A broad search strategy was chosen that used the subject heading of Self Harm and key words included in the sub headings of: suicide, paramedic, emergency, overdose, pre hospital mental health, ambulance, perceptions of care, and emergency. Manual searches of academic journals, grey literature, policy documents, procedures, clinical guidelines, and Government legislation were conducted.

\section{Confirming initial interest}

Given limited paramedic and pre hospital literature, all articles with a paramedic focus were selected for review and manually scanned for relevance and eligibility. The following criteria were used to select studies for analysis: 
a. The expressed a priori purpose of the study was to examine paramedic or emergency care for $\mathrm{SH}$

Or

b. A focus on education for paramedics

Or

c. Studies including decision making in relation to mental health in the pre hospital environment

And

d. Data were gathered from paramedics or emergency care personnel defined as doctors and nurses working in an emergency care setting

And

e. The studies were conducted using qualitative methods.

\section{Reading studies and extracting data}

To eliminate publications that were clearly not reports of qualitative research relevant to paramedics' and emergency staff perceptions of care for people who SH, a review of each citation title and abstract was conducted. Each study was subjected to a quality critique form based on a combination of CASP (2002) and Burns (1989). This included the CASP (2002) 10 questions to help you make sense of qualitative research and Burns (1989), which involved considering studies against the following standards (each with multiple criteria) for qualitative research: (a) descriptive vividness; (b) methodological congruence; (c) analytic preciseness; (d) theoretical connectedness; and (e) heuristic relevance.

\section{Determining how studies are related}

This involved open coding where each paper was read, findings were highlighted and compared for similarities, differences and questions regarding emergent phenomena. The concept-indicator model (Strauss 1987) was used in this process which relies on constant comparisons in the text. An indicator refers to a word, phrase or sentence, and a concept is a label associated with that indicator. Indicators were grouped together under concept headings, when another indicator did not generate new insights to a concept, it was deemed theoretically saturated and thus well grounded.

\section{Translating studies}

Within this stage axial coding was conducted; this involved categorisation where concepts were further translated, considering how they could be subsumed under higher-level metaphor headings.

\section{Synthesising translations}


Further synthesis was achieved through selective coding, which is defined as:

"explication of the story line" (Strauss \& Corbin 1998, p. 148). Intense analysis around metaphors was conducted, seeking to answer questions about when, where, why, who, how, and with what consequence?

\section{Expressing the synthesis}

Finally, theoretical constructions conceptualised the relationship among the three levels of coding, where theoretically saturated metaphors were woven back together into the narrative translation and presented as the final metasynthesis.

A truncated version of the coding book is presented in Appendix K.

\section{Results}

734 papers were retrieved, of which a final 12 met the inclusion criteria (Fig 8). It was the intention to review studies that examined perceptions of paramedic and emergency care personnel of those who $\mathrm{SH}$, however no such studies were found to have been published. Therefore, synthesis drew on a wide body of literature which involved conceptualisations within studies and across contexts. In-hospital studies with emergency doctors and nurses were found that explored care for people who SH. Selfharm was also considered in studies exploring paramedic or emergency staff care for patients within the wider context of mental health problems.

\section{Critique of the quality of the literature}

Significant debate exists about quality assessment in qualitative research which centres on the philosophical rationale for such assessment, and what criteria to use to inform judgements about quality (Campbell et al 2011)? This debate includes issues of ontology, epistemology and methodology, and it is therefore important to acknowledge the main positions taken in this thesis when considering the quality of qualitative studies reviewed in the metasynthesis. The interpretivist-constructivist epistemology and relativist ontology followed in this study has been previously presented in this thesis, which acknowledges the multiplicity of perspectives and truths (Strauss, 1987; Strauss \& Corbin, 1990, 1994, 1998). This perspective considers that there is no one truth, but 
that the external world is a symbolic representation, where both the interior and exterior worlds are created and recreated through interaction and there is no divide between external and interior world (Strauss 1993).

Extreme relativists argue that it is impossible to judge research against external criteria, whist some have suggested that the same criteria can be used to assess quantitative and qualitative research (Campbell et al 2011). Hammersley (1992) however proposed subtle realism as an alternative to this position, accepting that similar criteria apply, but suggesting that some methods of quality assessment in quantitative research may be adapted for qualitative studies. Hammersley (1992) argued that the issues of validity and relevance are core to all research. Validity relates to the clarity of the description of data collection and analysis; the researchers' sensitivity and reflection on the ways in which the data has been shaped and interpretations made. Relevance however considers whether the research adds to the knowledge and how they can be generalised to wider setting.

The Critical Appraisal Skills Program 10 Questions to Help you Make Sense of Qualitative Research' (CASP 2002) was used to appraise the quality of studies included in the metsynthesis. This was previously used in the systematic review earlier in the thesis and in other systematic reviews on care for people who SH (Taylor et al 2009, Saunders et al 2012). The systematic review in this thesis and Saunders et al (2012) used CASP (2002) to generate a numerical score to determine quality, however, this felt a very reductive method and not in keeping with the philosophical approach taken in the study. Taylor et al (2009) however, also used CASP (2002) but did not apply a numerical score, but rather used a strong or weak rating scale to report quality, which also sought to determine relevance to the purpose of the review. The metasynthesis reported in this thesis therefore rated the quality of studies based on a combination of CASP (2002), followed by each study then being subjected to further quality critique based on Burns's (1989) which involved considering studies against the following standards (each with multiple criteria) for qualitative research: (a) descriptive vividness; (b) methodological congruence; (c) analytic preciseness; (d) theoretical connectedness; and (e) heuristic relevance. Two of the review authors independently assessed the quality of studies using this method. 
The quality of studies included: one of low, six of moderate and five of high quality. The studies' methodologies included: ethnography, ethnomethodological, grounded theory, narrative accounts, hermeneutics and thematic analysis. The methods included: structured, semi structured and in-depth interviews, observational field work and surveys. Participants and practice settings included: Emergency Department (ED) and medical admissions doctors and nurses, paramedics and pre-hospital nurses. Studies were carried out in the UK, Sweden, USA, France and Australia.

Many of the moderate and lower rated studies (Squires and Mason 2004, Machen et al 2007, Cox et al 2006, Porter et al 2007, Tangherlini 2000) received lower ratings based on a lack of heuristic relevance, which is the value reflected in the reader's capacity to recognize the phenomenon described in the study, its theoretical significance, its applicability to practice situations, and its influence in future research activities. Whilst many of these studies included paramedics, they did not relate to mental health or $\mathrm{SH}$, and in discussions between the reviewers it was acknowledged their applicability was limited and may be considered a threat to achieving theoretical connectedness, and therefore provided limited contribution to theory development. This was in contrast to higher quality studies (Shaban 2005a, Roberts \& Henderson 2009, Ahl \& Nyström 2012, Nurok,\& Henckes (2009), where mental health or SH may not have been the focus of the study, but did feature within the text, thus adding to theoretical connectedness and providing contribution to theory development. Heuristic relevance of these higher quality studies also resulted in higher ratings due to a wide range of other factors, such as intuitive recognition where the reviewers immediately recognized the phenomenon being described and its relationship to a theoretical perspective in paramedic care for people who SH.

Shaban (2005a) was an example of a high-quality rated study, as it included paramedics, but also because of methodological congruence due to the rigorous development and presentation of a decision trail as advocated by Miles \& Huberman (1984). The theoretical framework suggested by Shaban (2005a) guided the research design, data collection, and analysis of the study, and used discourse historical case study as the primary methodology, applying ethnographic and discourse analytic techniques in data analysis. Shaban (2005a) incorporated a comprehensive array of material such as policies, procedures, clinical guidelines, pedagogical materials, standards, policies, and 
legislation. Theoretical statements were therefore linked to data and added to heuristic relevance.

Low and moderate quality studies included in the metasynthesis (Squires and Mason 2004, Machen et al 2007, Cox et al 2006, Porter et al 2007) also lacked justification of participant selection, the recruitment strategy and failed to outline in-depth description of the analysis process.

Studies included are presented in Fig. 9.

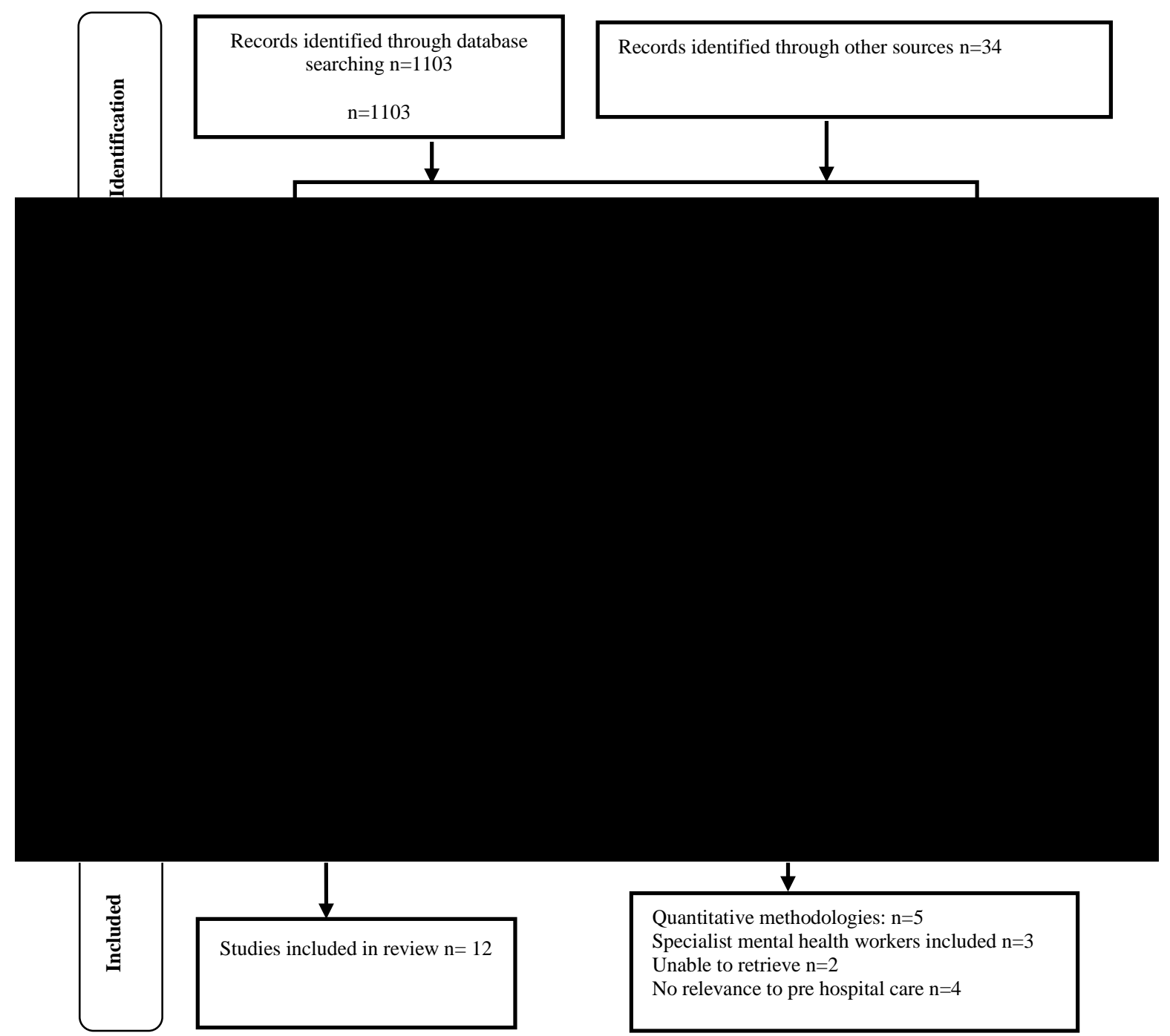

Fig 8. Moher, et al. (2009) Preferred Reporting Items for Systematic Reviews and MetaAnalyses: The PRISMA Statement. 
Fig. 9

Studies included in Metasynthesis

\begin{tabular}{|c|c|c|c|c|c|}
\hline Study & Study focus & $\begin{array}{l}\text { Method of } \\
\text { data } \\
\text { collection }\end{array}$ & $\begin{array}{l}\text { Methodology and } \\
\text { methods of data analysis }\end{array}$ & $\begin{array}{l}\text { Setting/ } \\
\text { clinician }\end{array}$ & Themes \\
\hline Anderson et al (2003) & Self Harm & $\begin{array}{l}\text { Semi- } \\
\text { structured } \\
\text { interview }\end{array}$ & GTM Charmaz (1995) version & $\begin{array}{l}\text { Nurses \& Dr's } \\
\text { E/D paediatric } \\
\text { medicine and } \\
\text { child and } \\
\text { adolescent mental } \\
\text { health }\end{array}$ & $\begin{array}{l}\text { Experiences of frustration in } \\
\text { Strategies for relating to your }\end{array}$ \\
\hline Hopkins (2002) & Self-Harm & $\begin{array}{l}\text { Observation } \\
\& \text { Semi- } \\
\text { structured } \\
\text { interviews }\end{array}$ & $\begin{array}{l}\text { Ethnography descriptive analysis } \\
\text { 'constant comparison' } \\
\text { (Hammersley \& Atkinson 1983) }\end{array}$ & $\begin{array}{l}\text { Nurses on } \\
\text { Medical } \\
\text { Admissions Unit }\end{array}$ & $\begin{array}{l}\text { The busy quality of such war } \\
\text { How this group of clients im! } \\
\text { Strategies nurses use to cope }\end{array}$ \\
\hline Tangherlini (2000) & $\begin{array}{l}\text { Story telling tactics amongst } \\
\text { paramedics }\end{array}$ & Interviews & Narrative account & $\begin{array}{l}\text { Paramedics } \\
\text { Pre Hospital }\end{array}$ & $\begin{array}{l}\text { Narrative explaining how the } \\
\text { important part in their tactica } \\
\text { relationships that structure th }\end{array}$ \\
\hline Shaban (2005a) & $\begin{array}{l}\text { Paramedic clinical judgment } \\
\text { of mental illness }\end{array}$ & $\begin{array}{l}\text { Ethnomethod } \\
\text { ological } \\
\text { using } \\
\text { discourse } \\
\text { linguistic } \\
\text { analysis, and } \\
\text { conversation } \\
\text { analysis. } \\
\text { Document } \\
\text { review \& } \\
\text { semi- } \\
\text { structured } \\
\text { interviews }\end{array}$ & $\begin{array}{l}\text { Ethnographic \& } \\
\text { Ethnomethodological } \\
\text { discourse historical } \\
\text { case study applying } \\
\text { ethnographic and discourse } \\
\text { analytic techniques in data } \\
\text { analysis. }\end{array}$ & $\begin{array}{l}\text { Paramedics } \\
\text { Pre Hospital }\end{array}$ & $\begin{array}{l}\text { Categories of mental illness. } \\
\text { training. Legislative, Policy a }\end{array}$ \\
\hline $\begin{array}{l}\text { Squires and Mason } \\
\text { (2004) }\end{array}$ & $\begin{array}{l}\text { Developing alternative } \\
\text { ambulance response schemes }\end{array}$ & Focus groups & Thematic analysis & $\begin{array}{l}\text { Pre Hospital } \\
\text { Paramedics }\end{array}$ & $\begin{array}{l}\text { A mismatch between policy a } \\
\text { conveyance decisions. }\end{array}$ \\
\hline Machen et al 2007 & $\begin{array}{l}\text { Expansion of paramedic } \\
\text { practice }\end{array}$ & $\begin{array}{l}\text { Qualitative } \\
\text { questionnaire } \\
\text { Semi- } \\
\text { structured } \\
\text { interviews } \\
\text { and focus } \\
\text { groups. }\end{array}$ & $\begin{array}{l}\text { Mixed methods Interpretive } \\
\text { approach (Denzin and Lincoln, } \\
\text { 1998). }\end{array}$ & $\begin{array}{l}\text { Nurses and } \\
\text { paramedics }\end{array}$ & $\begin{array}{l}\text { Involvement was a positive f } \\
\text { Staff felt confident in managi } \\
\text { knowledge and skills, and the } \\
\text { improved. Experienced incre } \\
\text { development. }\end{array}$ \\
\hline
\end{tabular}




\begin{tabular}{|c|c|c|c|c|c|c|}
\hline Cox et al (2006) & $\begin{array}{l}\text { Expansion of paramedic } \\
\text { practice }\end{array}$ & $\begin{array}{l}\text { Focus group } \\
\text { interviews }\end{array}$ & \multicolumn{2}{|c|}{$\begin{array}{l}\text { Content analysis (Bowling, } \\
\text { 2002). }\end{array}$} & Paramedics & $\begin{array}{l}\text { Perceptions of drivers for ch } \\
\text { Duty of care; perceptions of } \\
\text { preparedness for practice. }\end{array}$ \\
\hline Porter et al (2007) & $\begin{array}{l}\text { Ambulance crews attitudes } \\
\text { towards clinical } \\
\text { documentation and non- } \\
\text { conveyed patients }\end{array}$ & $\begin{array}{l}\text { Focus group } \\
\text { interviews }\end{array}$ & \multicolumn{2}{|c|}{ Thematic analysis } & $\begin{array}{l}\text { Paramedics } \\
\text { Pre Hospital }\end{array}$ & $\begin{array}{l}\text { Considerable ambiguity was } \\
\text { aware of the need to "cover } \\
\text { records, but expressed doubt } \\
\text { documentation. } \\
\text { There appeared to be two ma } \\
\text { were not completed. Firstly, } \\
\text { may have been unable to obt } \\
\text { patients who were intoxicate } \\
\text { Secondly, there were cases w } \\
\text { recognised their encounter w } \\
\text { dimension. These circumstan } \\
\text { monitoring by managers of w } \\
\text { completed, and a disinclinati } \\
\text { members to do what they reg }\end{array}$ \\
\hline $\begin{array}{l}\text { Roberts \& Henderson } \\
(2009)\end{array}$ & $\begin{array}{l}\text { Paramedic perceptions of their } \\
\text { role, education, training and } \\
\text { working relationships when } \\
\text { attending cases of mental } \\
\text { illness }\end{array}$ & $\begin{array}{l}\text { Clinical } \\
\text { database, a } \\
\text { survey and } \\
\text { three focus } \\
\text { groups. }\end{array}$ & \multicolumn{2}{|c|}{ Thematic analysis } & $\begin{array}{l}\text { Pre Hospital } \\
\text { Paramedics }\end{array}$ & $\begin{array}{l}\text { Role of paramedics managin } \\
\text { training, organisational cultu } \\
\text { relationships. }\end{array}$ \\
\hline Campeau (2009) & Paramedic scene-management & Interviews & \multicolumn{2}{|c|}{$\begin{array}{l}\text { Grounded Theory Constant } \\
\text { comparison }\end{array}$} & $\begin{array}{l}\text { EMS Canada } \\
\text { Paramedics from } \\
\text { rural, suburban } \\
\text { and urban } \\
\text { settings }\end{array}$ & $\begin{array}{l}\text { A theory was generated that } \\
\text { paramedics coordinate key st } \\
\text { "space control" at the scene }\end{array}$ \\
\hline Ahl \& Nyström (2012) & $\begin{array}{l}\text { The meaning of caring in pre- } \\
\text { hospital emergency care }\end{array}$ & $\begin{array}{l}\text { In depth } \\
\text { interviews }\end{array}$ & \multicolumn{3}{|c|}{$\begin{array}{l}\text { Hermeneutic philosophy of } \\
\text { Gadamer } \\
(1997)\end{array}$} & $\begin{array}{l}\text { Five dialectic themes: } \\
\text { Transportation or Care, } \\
\text { Acceptance or Rejection, } \\
\text { Participation or Exclusion, } \\
\text { Giving oneself over or callin } \\
\text { The Emergency Ward - Reli }\end{array}$ \\
\hline $\begin{array}{l}\text { Nurok,\& Henckes } \\
(2009)\end{array}$ & $\begin{array}{l}\text { Professional values and } \\
\text { the social valuation of } \\
\text { patients in pre-hospital } \\
\text { emergency work }\end{array}$ & Observational fiel & vork & $\begin{array}{l}\text { Grounded Theory } \\
\text { Constant comparison }\end{array}$ & $\begin{array}{l}\text { Pre Hospital } \\
\text { Paramedics in } \\
\text { New York and } \\
\text { Paris }\end{array}$ & $\begin{array}{l}\text { Emergency work is shaped b } \\
\text { identically prioritised and tha } \\
\text { framework that called a "fluc } \\
\text { Themes: } \\
\text { - } \quad \text { Social value } \\
\text { - } \quad \text { Technical value: The pr } \\
\text { - } \quad \text { Medical and surgical va } \\
\text { - } \quad \text { Intellectual value: the a } \\
\text { - } \quad \text { Heroic value: "the cour } \\
\text { - } \quad \text { Perfection and compete }\end{array}$ \\
\hline
\end{tabular}


Four interrelated metaphors were constructed capturing perceptions of paramedic and emergency care personnel caring for people with mental problems including those who SH: (a) Frustration, futility and legitimacy of care; (b) First contact in the pre hospital environment: talking, immediate and lasting implications of the moral agent; (c) Decision making in Self Harm: balancing legislation, risk and autonomy; (d) Paramedics' perceptions: harnessing professionalism and opportunities to contribute to the care of Self Harm.

(a) Frustration, futility and legitimacy of care

Caring for people who $\mathrm{SH}$ evokes experiences of frustration in practice, with a sense of futility around the effectiveness of interventions (Anderson et al 2003). This was articulated by a doctor in Anderson et al (2003) who said:

"When you've got a department or ward full of severe asthma, meningitis,...etc and then you've got a couple of young girls who have taken a cocktail of things . . . They cannot . . with our current resources . . . be looked after in the same way"

Neither asthma nor meningitis is dealt with in the same way, $\mathrm{SH}$ can be more damaging to health than these conditions, yet it appears to be of less importance, and there is a nuanced rationing of time and care. This was also found in Hopkins (2002) where one nurse participant said:

"Sometimes it can be frustrating if it is busy because you feel you can't give patients the time that is needed"

and:

"One girl often takes a lot of Paracetamol. When she comes in she has Parvolex treatment every time - you know, the antidote. I mean, you are just doing the same thing time and time again ... working in here, I have become cynical-you just get sick of it."

The studies presented a division between the deliberate nature of $\mathrm{SH}$ and acute medical conditions, along with a distinction being drawn between legitimate and illegitimate needs for care, with the former being viewed as having a greater entitlement to care. This was evident in comments from Nurok \& Henckes's (2009) field work in Paris and New York with pre hospital emergency services. They observed how social values and certain patient attributes influenced how patients traverse medical systems; emergency calls where there was an assumption of low 
social value or those considered to be less deserving were responded to slowly by paramedics with little conviction, and a lack of empathy by paramedics. The following case was observed:

"A call for a patient living in a housing project known to them, the team complains about being given another lousy case and starts driving to the call without much conviction. On arrival they find a patient almost unconscious. Upon seeing this, the paramedics pick up their pace rapidly administering Naloxone (a drug that reverses the effects of overdose). The patient regains consciousness and starts breathing normally. The team recognizes that the patient has probably overdosed on drugs and she is placed in the ambulance and transferred to the hospital without any display of empathy."

Nurok \& Henckes (2009) recognised three phases: At first the paramedics make a judgment of low social value and consequently show little interest. On arrival they recognize the patient is in extremis, which they can treat, the case then takes on higher value. Once lifesaving treatment has been successful, they focus on the overdose, which leads to a devaluation of the case, demonstrated by a lack of empathy toward the patient. In these three phases they argue a transaction occurs between judgments about the patient as a person and the technical dimension of enjoyable work.

Anderson et al (2003) found that nurses and doctors also made moral judgements about the value of a person's life. The risky behaviour of SH was seen as a potential waste of life, with young people having no respect for the dangers. Nurses and doctors saw their role as preservers of life, and $\mathrm{SH}$ being viewed as the opposite, and thus challenging the reasons they came into healthcare. One ED nurse reflected:

"I don't think they realise how potentially life threatening it is"

Anderson et al (2003) found the basis of frustration was the difficult feelings that whatever they do, nothing seems to work. Articulated by another nurse:

"I find it frustrating that I can't help ... I know of kids that have been heremy colleagues have said that have been regular attenders-self-harm-it's frustrating...I mean that is the frustrating part of it,"

Such futility of care was also found in the work of Hopkins (2002) where a nurse said:

"working in here, I have become cynical-you just get sick of it" 
(b) First contact in the pre hospital environment: The value of talking, immediate and lasting implications of the moral agent

In the Emergency Department (ED), Anderson (2003) found nurses and doctors wanted to engage and listen to people who $\mathrm{SH}$, however the environment posed a non-therapeutic option, as articulated by one doctor:

"This department isn't conducive to being able to talk to them in privateyou have got different priorities"

In contrast, Roberts \& Henderson (2009) conducted a study of the perceptions of paramedics regarding their role, educational training, and organisational culture when attending suspected or known cases of mental illness. One participant paramedic noted that:

"I was criticised for being a social worker because I would actually sit down and talk to them. I think it really depends on how the paramedic and society stereotypes individuals."

The criticism in this quote was suggested to have come from other paramedic colleagues and, along with the suggestion of stereotyping patients with mental health needs, it may also reveal something about the shared value paramedics have for calls to people with mental health problems such as SH. The study by Nurok \& Henckes (2009) presented earlier in this thesis highlighted the transaction of judgements which can occur with paramedics between the patient as a person, and the technical dimension of enjoyable work. Nurok \& Henckes (2009) also revealed that not all cases possess the same heroic value, suggesting that certain calls to emergency medical services, such as those for patients who do not require resuscitation possess no heroic value whatsoever, and are generally considered the non-valuable dirty garbage work that must be waded through in order to perform the desirable valued work of resuscitation. The criticism that the paramedic in Roberts \& Henderson (2009) received for sitting down and talking to patients who present with mental health needs may therefore be an indication of the lack of such heroic value these patients have to paramedics.

The paramedic environment poses a stark contrast, with opportunities to talk in private at the scene, or in the ambulance. Campeau (2009) highlights this, finding how in contrast to other healthcare providers, paramedics do not have predetermined work areas, but accept the location where they find a patient as their 'working area'. 
Campeau (2009) produced a grounded theory explaining how scene management is a dynamic social activity which includes sustaining social processes and antecedents of establishing a safety zone, trading off patient care and scene safety. These elements can be observed in other studies relating to paramedics and mental health. In Shaban (2005a) one paramedic said:

"I would have to say in my view most psychiatric patients I think would have been identified loosely under a dreadful term as 'mad people' and the police would have been called"

and:

"there is a fear of mental stigma, because I don't need to bother, they can either call the police, treat them as dangerous, and they don't particularly want to know"

The antecedents of establishing a safety zone and trading off patient care and scene safety, clearly come from a stated stigma toward mental health and the perceived danger mitigated by the social process of reducing uncertainty through social relations, and in this case involvement of the police.

Ahl and Nyström (2012) also found that the initial encounter with a pre hospital care provider is of vital importance, with the potential for satisfaction as well as dissatisfaction with care and outcomes. Patient's interviewed in the study by Ahl and Nyström (2012) initially regarded an ambulance as a means of transport rather than a place for care; when treatment was felt to delay care, frustration, and anger emerged, with a patient telling:

"I became frustrated... instead of taking me directly to the hospital they started fixing things... so I told them to hurry up"

This illuminates the notion that the initial impression of pre-hospital care and caring relations contains two different possibilities: acceptance or rejection. Feelings of safety often emerged in Ahl and Nyström's paper (2012), which highlighted that on arrival of the ambulance which was staffed by pre hospital nurses, a secure basis for trust and confidence was created. One pre hospital nurse told:

"Now you are my patient and to me you are the most important person in my life at the moment as a caregiver" 
This individual-oriented attitude of pre hospital care was found to be in contrast to other areas of the health care sector, with a patient telling how:

"In the ambulance I am the only one. At the hospital there are several of us".

This was the inverse to the frustration noted by a nurse in Anderson's work (2003) which stated: "you feel you can't give patients the time that is needed" because of competing demands.

The arrival of the ambulance is often the starting point for a caring experience (Ahl \& Nyström's 2012), and the first contact with a paramedic or emergency care worker following $\mathrm{SH}$ is of vital importance. This first contact can have a bearing on future acceptance or rejection of care; it may lead to avoiding emergency services in future SH and can determine the care and services they receive from then (RCPsych 2010, Ahl \& Nyström's 2012). The approach to care for people who SH from paramedics and emergency care workers can therefore have immediate and lasting implications for the patient. Within this context, paramedics and emergency care worker may be considered to act as a form of moral agency, which is a philosophical concept that describes the responsibilities of an individual in deciding whether to pursue an action or leave an action undone (Porter 2010). Moral judgments are based on commonly held notions of right and wrong, to do so on behalf of others, and to be held accountable for these actions (Angus 2003).

(c) Decision making in Self Harm: balancing legislation, risk and autonomy Shaban (2005a) examined paramedic accounts and constructs of judgment and decision-making about mental health patients. They found such patients to be categorised as presenting with violent, suicidal, drug-induced, or overtly psychotic behaviour. This is of importance because, whilst this paper explores $\mathrm{SH}$, the complexities of mental health legislation and making the distinction between such mental health presentations appears to be of concern to paramedics. UK guidelines call for support for paramedics (RCPsych 2006, NICE 2004) which may include access to approved doctors and social workers when assessing mental capacity and application of the Mental Capacities Act (MCA) (2005) and Mental Health Act (MHA) (1983). However this may not be happening in practice. Porter et al (2007) found in interviews with paramedics, that when patients declined to attend hospital, 
they struggled to get support. "Social workers were so thin on the ground" said one paramedic that trying to get hold of one was "a complete waste of time".

As for GPs:

"the doctor doesn't want to know, usually. Or we'll get in touch with the police. If it's in the patient's house, the police won't come out to the house, they won't come across the threshold, very often"

Porter et al (2007) did not specifically explore SH, however insights into decision making and mental capacity are of importance, and were interpreted in the current paper to articulate the challenge in dealing with people who $\mathrm{SH}$ and refuse care. Paramedics in the UK will involve police, social workers or doctors in attempts to apply the MHA (1983). Section 136 of the UK MHA (1983) allows for a patient to be detained for their own safety when in a public place, however, it is often inappropriately used in a patient's home, hence the tension that police will not come across the threshold. This tension was also articulated by a paramedic in Shaban (2005):

"I think both police and ambulance are in a very difficult position when the legalisation asks them to make value judgment calls"

Roberts \& Henderson (2009) acknowledge that although the police no longer routinely attend in mental illness cases in Australia, paramedics still call for their support in difficult cases. Luck and Townsend (2013) reported a case in Australia which highlights challenges the police also face in dealing with $\mathrm{SH}$. They attended a potentially suicidal man sitting in a van with a vacuum hose from his exhaust pipe into his window. After talking with the man and making inquiries they assessed he was not at current risk of SH but was rational and coherent and did not appear to be mentally ill. The man completed suicide later that day in his car via carbon monoxide poisoning. The deceased's partner sued, alleging the police were negligent and breeched their duty of care by not apprehending him under S10 of the Mental Health Act 1986. The court decided in Stuart v Kirkland-Veenstra [2009] HCA 15 that a duty of care should not be imposed, as the police officers did not have the requisite control over the risk of harm to the deceased (in Luck and Townsend 2013).

Porter et al (2007) also found a picture of anxiety and uncertainty about duty of care in ambulance crews, exhibited in the following comments: 
"If they say, 'I'm not going', we have no power to take them"

"We'd be done for assault"

"Either way, you're screwed, you are"

"At the end of the day, they have a right, their own rights, to refuse. Once they do, it's a minefield for us, then."

All of the studies surrounding paramedic management of mental health emergencies are from Australia (Roberts \& Henderson 2009, Shaban 2005a, Domino et al 1982, Shaban et al 2004, Shaban 2006), where there are significant differences in paramedic education and legislation. Despite this, there is similar detail of confusion revealed by paramedics in Shaban (2005):

"we are probably under equipped in so far as what we are doing under the Mental Health Act is putting us into a legal realm as such, as we are grabbing people under the Act, we may not do well if things actually went court."

Whilst the MCA (2005) and MHA (1983) are explicit in their direction, they still require enactment and interpretation in a given situation. Porter et al (2007) found confusion of ambulance staff over the process of deciding whether or not someone had the capacity to make the decision to refuse transport and treatment, and problems with its interpretation. One paramedic said:

"There are ten of us in the room and obviously we have all got different ideas if the patient meets the criteria".

Paramedic care for people who SH often involves on the spot decisions in emergency situations, and is clearly challenging. Wyatt (2012) explored professional judgments made by paramedics faced with such unique and non-routine situations, finding decision-making to be associated with development of tacit knowledge. The relationship of tacit knowledge with judgments made as part of expert practice was considered, finding that paramedics utilised multiple sources of information when making judgments. A rich interplay of factors was revealed contributing to the development of judgment-making, including experience within a particular context, along with reversion to tried and trusted rules or guidelines, and the capacity to play a hunch and trust in judgment.

(d) Paramedics' perceptions: harnessing professionalism and opportunities to contribute to the care of Self Harm

Studies of paramedics' perceptions of care have explored areas including expansion of practice and Myocardial Infarction (MI) care (Cox et al 2006, Machen et al 2007). 
One paramedic interviewed by Cox et al (2006) perceived other healthcare workers to be unaware of paramedics' professional responsibilities, telling how: 'the general ward nurse and general ward doctor, I don't think they completely understand what we do'. Despite this, opportunities are being captured to improve health through a whole systems approach in other areas of medicine. However, this opportunity is often overlooked, evident in Conlon \& O'Tuathail's paper (2012) where it is argued that:

"nursing services are at the core of the ED, and are usually the first healthcare professionals with whom a self harm patient comes into contact, providing triage, first aid and psychological support".

Whilst this might be correct, unless the patient self presents to the ED, the health professional that often gives care in the immediate aftermath of $\mathrm{SH}$ is the paramedic. Shaban (2006) recognises such an opportunity, stating that:

"the roles of paramedics and contributions they can make to the care of the mentally ill in the wider continuum of health care have not been fully recognised".

Tangherlini (2000) noted that part of the working day of paramedics consisted of telling stories about emergency responses. They found nurses and doctors engaged in a bitter "turf war" over the treatment of patients. One paramedic told how: "Nurses question the training and abilities of medics, while medics deride the competence of nurses". Paramedics' perceptions of their care are thus vital. Whilst no studies were found on $\mathrm{SH}$, studies in other areas identify a commitment to development, based on the quality of the evidence, ethical considerations and the unique opportunity to provide very early treatment (Cox et al 2006, Machen et al 2007). The most common concern that emerged in Cox et al (2006) was 'lack of ownership', participants believing new procedures had been imposed without involving 'front line' staff. Some paramedics felt they were being used to achieve targets, and their views were regarded as unimportant by policy makers. Squires and Mason (2004) similarly reported lack of ownership as a common finding in their study of attitudes to developing alternative ambulance response schemes, which could also act as a barrier to change. 
Paramedics identified attending to life threatening conditions as their first priority, dealing with the consequences of behaviour such as managing an overdose or physical trauma, and underlying mental illness was felt to be outside their skill level, as illustrated in the paramedic quotes in Roberts \& Henderson (2009):

"Role of paramedic: to deal with any medical aspect of treatment to a patient who may also have a mental illness... Presentation of overdose, management of overdose, management of any injury/self harm. Paramedics should not be for detention or psychiatric counselling"

The paramedics' safety was vital, especially when dealing with unpredictable behaviour:

"I think safety is of paramount concern with mental illness, possibly more so than with other complaints and certainly your preparation and awareness of potential or unpredictable situations is heightened when attending a case with mental health issues"

Paramedics felt the need to increase understanding and knowledge of different mental illnesses, treatment regimens available and focus training on what can be achieved in the pre hospital environment:

"Sessions available on specific mental health diseases and symptoms/management. More information on risk assessment of suicidelaggression. Information on negotiation with patient, while assessing to reduce the need for SAPOL Section 23 [legal detention]".

Roberts \& Henderson (2009) argued that limited training, time constraints, "frequent flyers" and a perception of limited treatment options influences the value placed on treatment of mentally ill patients. This was due to the need for extended treatment, as they recognised that even short-term outcomes would occur over days, rather than hours, and that paramedics may see their initial treatment as being ineffectual and of no value. A focus group participant in Roberts \& Henderson (2009) captures the importance of how people with mental health problems are perceived in society and by paramedics, stating that:

"[I was] criticised for being a "social worker" because I would actually sit down and talk to them. I think it really depends on [how the paramedic and society] stereotypes individuals" 
The criticism and judgement in this quote also reveals something about the value of caring for people with mental health problems; a sense that this is not part of their professional role as a paramedic, but rather the stereotypical notion that sitting down and talking to people with mental health problems is for social workers, not paramedics. Roberts \& Henderson (2009) did however recognise that SH now makes up a significant proportion of the work of paramedics, who also felt that they were "filling in the gaps" in providing services for people that an under-resourced and under-funded mental health system struggles to provide.

\section{Discussion}

Of those patients seeking health care services following $\mathrm{SH}$, many reported negative experiences, hostile staff behaviour, and lack of staff knowledge (MIND 2008, RCPsych 2008, RCPsych 2008, Houston et al 2003). This chapter sought to present findings from a metasynthesis of qualitative research relating to perceptions of paramedic and emergency care personnel for people who SH. However no qualitative papers specifically investigated paramedic care for SH. This may be influenced by challenges in conducting qualitative research in pre-hospital settings, as highlighted by Roberts et al (2013), who found that such challenges included the ethical implications surrounding the collaborative and participant-driven nature of qualitative research, along with the need to assure research does not impinge on staff time and workload. Despite this, the present study has explored emerging concepts and theory from wider contextual literature and synthesised them into a bigger whole in the narrative form. Whilst rich descriptions that illuminate themes in the original studies are lost, the metasynthesis offers a new story that identifies commonalities and themes across emergency care contexts and cultures in the care of those who SH.

It emerged within the metasynthesis that emergency care professionals saw themselves as preservers of life, and SH was counter to such professional motives. There was also a distinction made between legitimate and illegitimate needs for care, with medical cases taking a higher priority than those who SH. This was felt by $\mathrm{SH}$ patients who recalled being told they were wasting time and resources (Taylor et al 2009). Cook et al (2004) argued that many SH patients find themselves ignored by health professionals, not just because of negative attitudes toward them, but also 
because they are perceived as difficult to deal with. This distinction may influence the first contact with a patient who self-harms, with the potential for positive and negative outcomes, giving for a complex encounter which involves moral decision making.

Paramedics in Roberts \& Henderson's study (2009) felt criticised for sitting down and talking to patients who presented with mental health problems. Despite this, assessment in which the views of patients who SH are taken seriously and who participate in decisions about their care and treatment are highly rated (Taylor et al 2009). Above all, people who SH want to be seen by empathetic health professionals who can listen, be supportive and not judgemental (RCPsych 2010, NCCMH 2008, Burke et al 2008). Trust and an individual-oriented attitude in pre hospital care was also found within the metasynthesis to be valued by patients (Ahl \& Nyström 2012). This approach to care may reflect positive values, judgements and moral agency towards people who SH. However, care from paramedics and emergency care staff for people who SH appears to be complex and cannot easily be characterised as a positive, negative, good or bad.

Within the metasynthesis frustrations were revealed with caring for the same person who repeatedly self-harmed, and that staff could not give the care needed due to competing demands, along with the emergency department environment not being conducive to talking to patients and providing appropriate care following $\mathrm{SH}$ (Anderson 2003). These tensions reflect an element of moral distress, which is caused by situations in which the ethically appropriate course of action is known but cannot be taken (Elpern et al 2005). However, studies also reported negative and stigmatising approaches to care for certain groups of patients such as those with mental health problems, where paramedics could be 'criticised for being a social worker' (Roberts \& Henderson 2009) and people who require psychiatric care being identified by paramedics as 'mad people' for whom the police would be called (Shaban 2005a).

Sociologists have observed for a long time that certain personal patient attributes are highly valued by carers and influence how patients traverse medical systems (Dodier 
et al 1997). This is of importance in paramedic care for people who SH as paramedics are often the first health professionals encountered by a patient following $\mathrm{SH}$, and this interaction may influence acceptance or rejection of care, determine which services patients receive from then on, whether the police are involved (which could result in detention under the MHA 1983) or lead to patients' avoiding emergency services in following SH in the future (RCPsych 2010, Ahl \& Nyström 2012). Within the metasynthesis, studies revealed that the technical elements of physical care such as resuscitation appear to take on a higher (heroic) value to paramedics and emergency care staff than non-valuable dirty garbage work that must be waded through in order to perform the desirable valued work of resuscitation (Nurok \& Henckes 2009). The case of patient whom had overdosed in Nurok \& Henckes (2009) did indeed reveal the transaction of judgments made by emergency care staff about the patient as a person and legitimacy of care versus the technical dimensions. Studies also revealed how patients presenting with physical conditions seem to take on a higher legitimacy than people those in need of care following $\mathrm{SH}$ (Hopkins 2002, Anderson 2003). If indeed caring for people who SH is indeed viewed in this light by paramedics and emergency care staff, this needs to be fully understood and addressed urgently.

Future policy and education would benefit from recognising the perceived primary role of paramedics in dealing with the physical consequences of SH. Despite this, in Kilner (2004), mental health education was recognised to be of greater priority by paramedics than trauma and resuscitation, this is echoed by Shaban (2005a), and specifically risk assessment of suicide (Roberts \& Henderson 2009). The content of such education may include patient centeredness, caring, empathy, valuing life, professionalism, non-judgemental and non-discriminatory attributes, which also ranked in the top six desirable qualities in the paramedic in a study by Kilner (2004) and are the very attributes suggested by policy and guidelines for $\mathrm{SH}$ (Steen et al 1997, RCPsych 2010).

Policy and guidelines advocate that ambulance staff should have access to crisis resolution teams, approved doctors, and social workers regarding the assessment for those who SH (RCPsych 2006, NICE 2004, Burke et al 2008). The reality however is 
that paramedic crews find difficulties in decision making around mental health legislation (Shaban 2005a, Porter et al 2007). The rights of personal autonomy for those who SH in the UK are enshrined in the MCA (2005), but despite this, paramedics report anxieties over mental capacity (Roberts \& Henderson 2009, Shaban 2005a, Porter et al 2007). Paramedics likewise report difficulties and anxieties application of the MHA (1983) (Roberts \& Henderson 2009, Shaban 2005a, Porter et al 2007), even in Australia where paramedics have powers to detain under their MHA (Shaban 2005a). Enactment of MCA (2005) and MHA (1983) can therefore lead to a switch from respecting a patient's autonomy to a more paternalistic stance of acting in the patient's best interest. This has implications for future care as RCPsych (2010) revealed that patients had avoided services for fear of being detained under the MHA (1983) as the person's actions in seeking services might bring them to the attention of the police and to a place of safety.

Policy and guidelines highlight the professional responsibilities and clinical opportunities of paramedics in the care of people who SH (RCPsych 2008, RCPsych 2006, NICE 2004) and understanding the perceptions of paramedics was deemed vital to harnessing this opportunity. There was a perception among paramedics that healthcare workers were unaware of this potential. A lack of insight of the potential contribution of paramedics in SH care however may be because paramedics only gained professional registration in the UK in 2001 (Health Professions Order 2001). Whilst the professional responsibilities and clinical opportunities of paramedics are slowly being recognised in managing $\mathrm{SH}$, there appears to be fragility to this recognition. Some paramedics perceive their primary role as transportation and attending to life threatening conditions as their first priority; mental health was viewed as a secondary consideration. This is a significant finding and reconciling this position considering current and future policy and innovation, with the professional journey that paramedics are undergoing needs further consideration.

\section{Limitations}

An attempt was made to clearly described the search terms used. However, on further reflection, there were possible constraints which may have been imposed on the 
metasynthesis by the selection of search terms. For instance, the term perception can be defined as:

"a mode of apprehending reality and experience through the senses, thus enabling discernment of figure, form, language, behavior, and action. Individual perception influences opinion, judgment, understanding of a situation or person, meaning of an experience, and how one responds to a situation" Munhall (2008 p.606)

This may have limited the retrieval of papers which may have used other terms such as paramedics: experiences, view, opinion or understandings of care for people who $\mathrm{SH}$. The search terms may therefore have benefitted from being broader enough in scope to capture all the relevant data yet narrow enough to minimize the capture of extraneous literature that may result in unnecessary time and effort being spent assessing irrelevant articles.

The study employed systematic methods to identify and appraise published research and has found limited literature related to paramedic and emergency care. It therefore relied on synthesis of findings across practice and professional contexts. There was a possibility that as the main researcher was a paramedic, interpretations were made through a paramedic lens and might have an effect on the findings. Despite this, we believe we have been transparent about our methods and upheld trustworthiness of data in order to enable researchers to follow our audit trail.

\section{Conclusion}

A limited number of investigations have enquired about the patient's perspective of SH care yet, despite dissatisfaction with this care, few have sought to reveal the perspective of caregivers, and none have investigated paramedic care. Whilst methods have been rigorously applied, this study has relied on constructivist interpretations and translations of paramedic studies from a wide range of practice contexts. It has revealed SH to be a common yet complex professional, clinical and social interaction. The themes in this paper have highlighted potential areas for development for paramedic and emergency care, and this work will be used to evolve our understanding of potential contributions to the care for those who SH and to inform practice, including a tailored educational intervention for paramedics. 


\section{Chapter Six - Results}

\section{Introduction}

Previous chapters provided an introduction to the study and considered the context of paramedic care for those who SH. A wide range of areas were presented, including the scale of SH and the role of paramedics in providing care to this patient group. The aims and objectives were presented, along with the research design, methods, methodology applied to achieve these aims and objectives. The metasynthesis of qualitative literature and systematic review of quantitative literature highlighted the lack of published literature pertaining to paramedic care for people who SH. These reviews of the literature also served to stimulate theoretical sensitivity and assist in the development of the interview guide.

In this chapter the results of the interviews with paramedics are presented, a version of which (Appendix L) has been published in the journal Plos One (Rees et al 2018)

From twenty-six people who expressed an interest in the study eleven paramedics were included in the final sample of study participants. These included the two pilot interviews, as there were no major changes made to interview guide or sampling strategy. The range of experience of participants was between one and thirty-eight years in frontline pre-hospital care. The cohort comprised three women and eight men; six had completed degree-level paramedic development courses and five had undertaken traditional vocational training. Paramedics self-reported whether they worked in rural or urban areas; five paramedics worked in urban areas and six worked in rural areas. Two of the participants were interviewed twice to further explore insights revealed from initial interviews, four did not respond to requests for follow-up through member-checking, despite two follow-up requests, while seven did agree to follow-up on their interviews through member-checking. The paramedic participants will be referred to in the thematic presentation of findings below as Paramedic (Para) 1 to Paramedic 11. Code numbers are based on the order in which they were interviewed, with one being the first to be interviewed. 


\begin{tabular}{|c|c|c|c|}
\hline Gender & $\begin{array}{l}\text { Male } n=8 \\
\text { Female } n=3\end{array}$ & & \\
\hline $\begin{array}{l}\text { Years EMS } \\
\text { experience }\end{array}$ & $\begin{array}{l}1-3 \text { years } n=3 \\
3-10 \text { years } n=4 \\
>10 \text { years } n=4\end{array}$ & & \\
\hline Age range & $\begin{array}{l}\text { Up to } 18 n=0 \\
19-26 n=3 \\
26-45 n=7 \\
46-55 n=0 \\
56-60 n=1 \\
\text { over } 60 n=0\end{array}$ & & \\
\hline \multirow[t]{3}{*}{ Religion and Belief } & Christian $\mathrm{n}=5$ & Hindu $\mathrm{n}=0$ & Other $n=0$ \\
\hline & Muslim n=0 & Jewish $\mathrm{n}=0$ & None \\
\hline & Buddhist $\mathrm{n}=0$ & Sikh $n=0$ & $\begin{array}{l}\text { Prefer not to say } \\
n=6\end{array}$ \\
\hline $\begin{array}{l}\text { Paramedic } \\
\text { educational } \\
\text { development }\end{array}$ & $\begin{array}{l}\text { Higher Education } \\
n=6\end{array}$ & $\begin{array}{l}\text { Traditional } \\
\text { Paramedic } \\
\text { Training } n=5\end{array}$ & $\begin{array}{l}\text { Specific SH } \\
\text { training/Education } \\
n=5\end{array}$ \\
\hline $\begin{array}{l}\text { Geographical area } \\
\text { in which paramedic } \\
\text { works }\end{array}$ & $\begin{array}{l}\text { Urban } n=5 \\
\text { Rural } n=6\end{array}$ & & \\
\hline
\end{tabular}

Fig. 10 Characteristics of study participants

A Grounded Theory was developed of 'Wicked Complexity in Paramedics' care provided to people who Self-harm'. This included the Basic Social Process of: 'Decision making in a context of risk'. These both emerged through the coding process, which used six categories to describe paramedics' perceptions of caring for people who SH. They comprised: Context; Judgements and values; Isolation and System Failure; Managing Risk; Competence at the boundary of mental and physical health needs; Professional, Legal and Ethical Tensions. Appendix M presents a truncated representation of the coding book, and the interrelationship within codes.

\section{Categories}

\section{Category 1: Context}

Paramedics reported the importance of the context of the encounter with people who $\mathrm{SH}$ which related to the patient, the incident or the crew. The context within which $\mathrm{SH}$ takes place is of importance as it may influence how the paramedics behave and respond towards the patient and others. The context of the case is also important to paramedics and their colleagues as ambulance work is unique in that care is not 
provided in a controlled setting such as hospital, but rather in public places and people's homes, which can introduce vulnerabilities and risks to paramedics and patients. Three important processes of care emerged, which included pre-arrival, onscene care and the journey to hospital. The pre-arrival phase included frustrations felt by paramedics at being called to people who $\mathrm{SH}$ and being misdirected from other incidents perceived as more serious (crew). This phase was also influenced by who made the call (incident), where it was felt that the call was often made by somebody other than the person who had self-harmed, which may be the family, or when the patient was intoxicated with alcohol may be a bystander. The pre-arrival phase also appeared to influence care for people who SH, due to busyness and tiredness of paramedics, which appeared to amplify their frustration and unsympathetic attitudes. As the call was often made by someone else, and the paramedics admitted to negative attitudes, refusal of care was a potential outcome.

Paramedics reported feeling a sense of frustration towards a person who had selfharmed, even before they had arrived at the call. They reported how they felt they could be dealing with more deserving calls, as there were not an infinite number of ambulances available. Paramedic 8 pointed out that:

"Because are they taking us away from something more urgent? Or that we feel is more urgent" (Para 8)

Paramedics were not in the position to know whether there was someone or something more urgent needing their care. However, there was a sense of a losing the availability of an ambulance to respond to other calls because they were committed on cases of $\mathrm{SH}$; the view that these appear to them to be of lower worth than more life-threatening calls was common. These issues instilled frustrations in paramedics even before they attended calls to people who SH.

Paramedics in our study pointed out that it was rarely the patient who called for an ambulance following $\mathrm{SH}$, but rather a concerned friend, member of the public or someone else, as revealed by Paramedic 2 who said:

"She said [the patient], I didn't call you, the other people called [the bystanders].”(Para 2) 
Calls for SH made by someone other than the patient was deemed to be especially the case when the SH was conducted in a public place. Calls such as these could also involve alcohol, and patients found in intoxicated states. Paramedic 6 tells how:

"There's normally a member of the public who passes and phones the police." (Para 6)

Family members and friends were identified by paramedics as the first ones often turned to by the patients following SH. It was usually the case that these family members were the ones to make the call for an ambulance following being made aware of the SH. As descried by Para 7:

"it's quite often it's a relative or a friend that they'll call first and say, you know, "I've done something really stupid." And then that relative or friend actually calls the emergency services" (Para 7)

And Paramedic 12:

"He'd left a text message to one of his friends who called the police and the police then also included the ambulance service and we turned up at the same time." (Para 12)

This pre-arrival context often resulted in patients refusing care, as they neither wanted this care in the first place, nor called for help from the ambulance service. Reluctance for care from the patient was compounded by reports of unsympathetic attitudes towards $\mathrm{SH}$ by paramedics who reflected how they were sometimes primed with negativity towards a person they were responding to following SH. This may be due to stigma and preconceptions being amplified when responding to $\mathrm{SH}$ within the context of busy periods, when the paramedics themselves were tired. This could then affect the care they provide, Paramedic 4 commented:

"Sometimes we are so browbeaten ourselves, or caught up in our own problems, overworked, underfed, whatever reason. These people are treated as a bloody nuisance." (Para 4)

When 'on-scene', these contextual, antecedent factors continued to influence care, although crews now emphasised the importance of patient and incident related influences. Contextual factors faced when on scene included the severity of injury, presence of others such as the family, police colleagues or bystanders, weapons, and alcohol impairing a patient's capacity and decision-making. Paramedic 9 remarked: 
"Alcohol can affect somebody's judgement, their mood, and you know, the way that they see things, and then they can be quite easily sort of wound up." (Para 9)

And added:

"Yeah I've been to one that's got a carving knife and was slashing at his thigh. And there was some police there waiting for a Taser Unit to come and take control." (Para 9)

Such encounters involve risks to patients, paramedics and others. These included the risk of suicide when a patient who has self harmed is not conveyed to hospital and risk of litigation towards the paramedic. These issues of risk are discussed further in the category managing risk, however risk of aggression and the role of substance misuse, drugs and alcohol were perceived to be important contextual factors in caring for people who $\mathrm{SH}$, which was deemed by paramedics to be influenced by the environment and context in which the SH takes place. In the pre-hospital environment, many contextual factors can influence care which will not be present in other care environments such as the controlled setting of a hospital. Paramedic 3 recalled an encounter where a woman had harmed herself with a knife. She was lying in bed in a small box room, and when he approached her he told how:

"I looked down and the patient had rolled over and she had a knife in her hand, but in like a dagger type action" (Para 3)

Paramedic 3 then went on to tell how the patient was pulling him towards her and his colleague was pulling backwards by his shoulder. Paramedic 3 reflected how:

"I obviously, put myself directly in danger there, I had no way of getting out of that room. I was basically trapped, you know, I was you know, she was between the door and me" (Para 3)

Many other contextual issues were recalled by paramedics in our study. Paramedic 6 recalled the case of a patient he regularly attended for $\mathrm{SH}$, and how the setting was used by the patient to communicate their need for help:

"I had spoken to her about the sort of sitting on the bridge, why the bridge? And she described the bridge as being a place that there's somebody there that will help her. There's normally a member of the public who passes and phones the police. Although I don't understand it, it is nice that she's going to a place that somebody's able to alert somebody to come and help her" (Para 6) 
The context of the SH encounter was also felt to be influenced by the location and surrounding geography. Rural paramedics felt they encountered less aggression in the cases of SH they attended than their colleagues working in cities, whom they felt encountered much more abuse. Paramedic 9 who was a rural paramedic said:

"Erm, people call us out and they say, "Well thank you for coming." That happens very often. We get very little abuse in this part of the world. I speak to paramedics in the cities and they say they get a lot of abuse" (Para 9)

It was also recognised by rural paramedics interviewed that paramedic work in an urban context could result in many more encounters of SH due to the busyness. Such busyness in the urban setting was cited by one such rural paramedic as a potential reason for poor attitudes towards $\mathrm{SH}$ in his urban paramedic counterparts:

"they (urban paramedics) are busy and they know that when this job is finished and got a next one? I don't know. Is it their workload that makes them like that?" (Para 6)

$\mathrm{SH}$ in a rural context was however perceived by paramedics to be more severe or lethal. A paramedic that had worked both urban and rural said:

"I've certainly had more successful self harmers in the rural community than I have in the urban community. And I would say urban self harmers are, if you want to put them at one scale, are more superficial than sort of rural." (Para 2)

And:

"There seems to be more intent to harm their life rurally, it always often seemed to be a lot more serious, where you'd get more minor self harm regular repeat offenders in the city centres, which we regularly, we regularly go to" (Para 2)

Paramedic 7 pointed to the influence of others who may be present. Many scenarios were described, including heightened and aggressive events of on-going $\mathrm{SH}$, to more melancholic circumstances with much sadness, shame and embarrassment.

Paramedics had to make on-the-spot judgments in such contexts which ranged from calling for police support to providing one to one care where the presence of relatives could be problematic as they could get in the way of assessing and treating the patient which may require discussion over very personal and emotive details of the $\mathrm{SH}$, which the patient may not wish their relative to hear. Paramedic 11 explained how: 
"It depends who's called the ambulance, you know, if it's a relative or something it can be difficult." (Para 11)

Paramedics found themselves in an awkward position when relatives had called for the ambulance, which was often the case. They conveyed the notion that the patient could be embarrassed, and that relatives could get in the way of care, as described by Paramedic 8 and Paramedic 4:

"A lot of Self-Harmers I've had, you know, some will want relatives to go with them, but the vast majority won't due to embarrassment of the situation that they find themselves in." (Para8)

"I have on occasion, you know, got my colleague to stop the vehicle if we've got a relative, if I think that there's an increase in distress in the patient, and put the relative in the front, and that's generally when we find that your patient will talk to you more about the problems they find. They just don't want friends/family knowing what they're going through." Para 4)

These two quotations highight how the presence of relatives could be problematic, and also point to important implications for delivering care. For instance, such shame and embarrassment may result in paramedics being prevented from gathering all the information they need to make an assessment and manage them appropriately. Shame in the presence of relatives may also make it less likely for patients to be left at home with relatives, especially in the absence of a full understanding of the situation prior to the $\mathrm{SH}$.

The context of the journey into hospital presented many opportunities and challenges when caring for people who SH. Paramedics spoke of their vulnerability in the context of providing care alone in the back of the ambulance:

"I've had people react badly to, erm, my comments in the back of an ambulance, or get quite aggressive because I've said to them, "Why?" (Para 5)

Paramedics interviewed reflected on the context of caring for people who SH when travelling long distances into hospital. It was recognised that this time in the back of the ambulance was a unique position where the paramedic and patient would be alone. Paramedic 10, who was very experienced, thought this served as a good opportunity to provide one to one care, telling how:

"We decided to remove him from that scene and take him to the back of the ambulance where we felt care might be more appropriate. When we got in 
the back he started opening up a bit more, very chatty guy, very, very nice man. We spent a little while with him just sort of getting him to calm down before we went anywhere, did all the normal obs, all that stuff and tried to help him sort of tell us what he--, what was going on in life, what was--, he felt that was the problem and sort of see if we could help him get down the right pathway, what--, we talked about what he had accessed, so we talked about him trying to go through the GP. "(Para 10)

Conversely, Paramedic 6, who had only been a paramedic for two years told how:

"that left me and this person in the back of the ambulance. The person was very quiet, very sedate, kind of crunched up on our trolley. And I didn't really attempt to engage anymore because I didn't really know what to say, I didn't know what were the right things to say to her. And of course you, you worry about making the situation worse, you worry about the woman suddenly saying, "No, I don't want to go," and, "Stop the ambulance." (Para 6)

The context in which paramedics care for people who SH is therefore unique, and unlike that of any other care provider. The three phases of care reported above encompass many contextual factors which have the potential to be both positive or negative aspects of caring for people who SH.

\section{Category 2: Judgements and values}

Paramedics in our study revealed much about the personal aspects of the relationship between paramedics and patients who SH. This included a range of attitudes and preconceptions towards people who SH. Paramedics reported how the majority of their colleagues displayed negative attitudes towards $\mathrm{SH}$, and whilst most interviews included stigmatising and judgemental attitudes, some individuals expressed both positive and negative judgements. These attitudes were reported to be influenced by busyness, the frequency of encountering SH and responding to the same patient time and time again following SH. Paramedic 2 told of his surprise on joining the service to discover the number of SH patients he encountered in his daily work with the Ambulance Service

"that was a big surprise to myself, I still say it now, when I joined the ambulance service the amount of self harm acts to be honest, the overdoses that we were attending on a regular basis. It would be two, three a night working in the city centre. And it was a bigger shock to me to be perfectly truthful, when I joined the Ambulance Service ten years ago, the amount of self harm. And it continues to this day that we deal with on a daily basis is quite large." (Para 2) 
All of the paramedics interviewed reflected on the high number of calls for people who SH. Paramedic 4 who worked in an urban area said:

"I would think it would be unusual for you not to get one self harmer in a---, primarily on a night shift." (Para 4)

Among these were patients who "become what we call regular" (Paramedic 3) - the people who are attended repeatedly by crews for $\mathrm{SH}$, who become familiar with them as individuals. Sophisticated encounters and relationships were revealed with people who SH and become regulars. Paramedic 7 gave an account of one regular (whom he was on first name terms with) where the patient:

"Opens his neck up and that is bloody scary when he does that, there's blood everywhere like and he's bloody hysterical... he has learned not to be awkward with us mainly because we are not awkward with him... We know him quite well... I mean he's just a person with issues" (Para 7)

Despite frequently encountering $\mathrm{SH}$, often with the same individuals, paramedics admitted to internal tensions they experienced with people who $\mathrm{SH}$, and could not understand why people SH. "I don't understand, inflicting pain on yourself" said Paramedic 2, and for some paramedics, the frequency with which they attended the same caller led to a less sympathetic approach:

"If you saw the same patient over and over and over again, and it's the same thing, you know. You can sort of, you know, get less sympathetic towards it like, you know, you can be more sort of hardened ... Especially if you're having an off day yourself ..." (Para 9)

Paramedics further articulated this complexity of their attitudes towards people who $\mathrm{SH}$, which were not as simple as being either positive or negative. Paramedic 9 went on to say:

"if you're having an off day yourself, it depends how your mood is yourself as well, like you know, so I think it would be, you know, the sort of, the straw that breaks that camel's back as it were like" (Para 9)

The frustration and lack of understanding felt towards those who regularly $\mathrm{SH}$ sometimes led to paramedics using pejorative terms when referring to them, such as “a regular" (Para 4), "self harm regular repeat offenders" (Para 6), and Paramedic 7 
told how some colleagues would have the attitude that "We're out to this idiot again” (Para 7)

Paramedic 3 told how:

"you know, they're a bloody nuisance. They've overdosed, they've cut themselves, they've tried to hang themselves, albeit half heartedly." (Para 3)

Whilst paramedics in our study used such terms, most did not admit to holding such negative attitudes towards people who SH but did report how these attitudes were common amongst other paramedic colleagues. For instance, Paramedic 1 recalled how:

"a colleague basically told her to pull herself together, sort herself out, stop wasting our time" (Para 1)

Paramedic 4 provided an account of how such judgements could impact on care, where a person was treated following $\mathrm{SH}$ and received the minimal amount of care, with limited communication:

"You probably would find that no-one's ever going admit it obviously but you'd probably find that they put them on the back of the ambulance; they've dressed the wound; they've taken their obs. That's probably the last sentence they'll get out of the paramedic or the patient on the way to hospital" (Para 4)

Paramedics in our study attempted to explain why such negative attitudes may be generated in themselves and their colleagues, with Paramedic 9 stating that:

"You tend to sort of, you know, if you saw the same patient over and over and over again, and it's the same thing, you know. You can sort of, you know, get less sympathetic towards it like, you know, you can be more sort of hardened and think, you know. Especially if you're having an off day yourself, it depends how your mood is yourself as well, like you know, so I think it would be, you know, the sort of, the straw that breaks that camel's back as it were like" (Para 9)

Despite this, all of the paramedics interviewed distanced themselves from such negative and stigmatised views as emphasised by Paramedic 8:

"it's a very misunderstood sort of--, misunderstood time waster sort of attitude that a lot of paramedics have got. Which personally I find unacceptable and when you chat to people and say, "Well have you thought about being in their shoes for just five minutes and see how dealing with their life?" And they always come back, "No but they shouldn't be doing it." (Para 8) 


\section{Category 3: Isolation and system failure}

Paramedics felt that increasingly they are the first point of contact for people who have suicidal thoughts, who SH, or those who die by suicide; "with self harm I think that we are often the first medical port of call these people see" (Para 8). They perceived there to be failures elsewhere in the health system which led to them being contacted for mental health related issues, and that serious mental health crisis may have evolved from lack of access to earlier support. Paramedic 2 recalled how a person he attended with multiple mental health problems had tried accessing mental health pathways prior to an emergency call for a suicide attempt:

"he had gone to his GP, he'd tried all that sort of stuff. He was desperate for help. Unfortunately he didn't feel that the system was quick enough for him. So he tried to take his own life by hanging." (Para 2)

When faced with dealing with people who SH they felt ill equipped and unsupported, as there was lack of assistance in terms of referral options for people who $\mathrm{SH}$, and a care system which failed to provide appropriate care, leading to a sense of isolation. Paramedic 9 commented:

"We don't necessarily access the right care for them, where we're going to... we should be seeking specialists, maybe taking them to the specialists rather-, and referring them rather than having to go through a busy emergency department, through busy GP surgeries." (Para 9)

Tensions emerged, particularly when people refused care, which exacerbated paramedics' concerns for patients taking their own life. In such circumstances, they felt a lack of control over the situation, and left with a sense of isolation. Paramedic 4 stated:

"You can't get hold of mental health practitioners to be able to help you, the lack of advice and support...Shouldn't there be a facility that we can refer directly to a mental health crisis team unit, and be able to go directly there instead of $A \& E$ ?" (Para 4)

The challenge of dealing with somebody following SH and refusal of care, with few support or referral options was further articulated by Paramedic 2, who when questioned about how it felt to be faced with this situation, responded: 
"It does make you feel lonely. It makes you, erm, it makes you feel annoyed, but not at the person that you're treating, but at the system that we currently have." (Para 2)

Paramedics reported being unable to access appropriate care, including Crisis Resolution Teams which can provide a rapid response and assessment for people experiencing a mental health crisis at home or in the community. Paramedic 6 , for example, explained:

"Phoned [ambulance] control, phoned GP out of hours who said she needs to go in but can't force her, Police tried ward, who wouldn't take her cause she'd been drinking, called crisis team ... forget it." (Para 6)

Paramedics provided accounts of the context of follow-on care for people they encounter who $\mathrm{SH}$, covering the referral options available, the destination of care, if the care was voluntary, coerced or forced upon the patient. As discussed earlier, in most cases their only option was to transport the person to the ED, and whilst they acknowledged some individuals may have injuries or medical problems which required ED treatment, they did recognise that for many, who had no medical problems appropriate for ED care or minor injuries, this may not be the best place for them. Powerful accounts were provided of the uncomfortable position they founded themselves in by not being able to offer what they felt was the right care.

The options and process of care following the initial encounter were felt to have a significant influence over the care interaction. When the decision had been made to go to hospital, the paramedics talked of their aim to get the patient into the back of the ambulance where they were more comfortable with the care context and could talk one-to-one with the patient:

"I told her that she'd be looked after well, and all that kind of stuff just to get her out to the ambulance" (Para 11)

The interviews revealed the developing of a personal relationship between the patient and the paramedic on the journey into hospital. Earlier, in the category Context, Paramedic 10 reflected on a case where talking to the patient during a long journey to hospital in the ambulance was deemed a positive aspect of care. The same patient “started opening up a bit more ...very chatty guy” said Paramedic 11. Such 
relationship and building of human contact appears to helps break down any mutual misunderstanding and increases the chance of the patient getting appropriate care.

Despite such accounts of the positive aspects of caring in the back of an ambulance, the main conern of the paramedics was to get the patient who had self-harmed into the ambulance to protect themselves and the patient. By ensuring the patient went to $\mathrm{ED}$, paramedics felt there was less risk of the patient dying by suicide, them being criticised should this happen. Paramedics did not believe that ED was always the best place for a patient following $\mathrm{SH}$, but felt it was their only option, where a patient could be further assessed and treated. Paramedic 1 summed this up position, and told how he was even prepared to lie to a patient:

"My aim when I got there was to get her in the truck and get her to hospital, and then I can wash my hands of it 'cause I don't know what to do, I haven't got another solution. So I lied to this lady, I told her that we'd go to the hospital, she'd be seen by a psychiatrist there, and they would decide on the best course of action, maybe it might mean you might need to go into somewhere for some therapy and stuff for a bit, but you'll be looked after. Knowing in my heart that she'd probably be stuck in some cubicle for all night, 'cause this was the evening, and there probably wasn't anyone going to come because the on-call psychiatrist was in XXXX [hospital; name removed for anonymity purposes], and apparently they very rarely leave XXXX [hospital], and she'd be stuck there all night on her own before being transferred to XXXX [hospital] or somewhere like that. But of course I didn't tell her that". (Para 1)

When the journey to hospital was over, Paramedic 6 remarked on the transition of care between the ambulance service and the ED. Paramedic 6 explained how the opportunity to talk to the patient on a one-to-one basis in the ambulance contrasted with the transfer into the chaotic ED environment:

"The Emergency Department is often busy, noisy, can be confrontational. Staff don't have time to deal with a patient on an individual basis, which is what's required." (Para 6)

Paramedics were not comfortable with the situation where patients in such distress were taken into the chaotic ED environment, but they recalled many times how they had no other option. 
There were many examples of perceived system failures provided by paramedics. Powerful accounts were given of patients who had self-discharged then later died by suicide, where the system was deemed to have failed them. Paramedic 4 told of a relative of his, whom he had also attended in his professional capacity following alcohol abuse. He explained how:

“The Crisis team won't come out 'cause he's been drinking” (Para 4).

Paramedic 4 recalled how he had sought numerous avenues of support for the individual, and finally he said:

"So in the end I had a chat with the doctor again and he said... basically his family have said that leave him calm down, leave him to sleep it off, and I left him lying face down head to the side, patent airway [open or clear airway], "Okay, if there's any problems give me a call." Half past seven that night he'd arrested (Heart stopped) (Para 4).

This was a distressing personal account, and all of the paramedics reported the potential for $\mathrm{SH}$ or depression in themselves, many told of family members or friends who had self-harmed. This link to their personal life and friends and family was pointed out by Paramedic 4, who paraphrased information provided to him during consenting:

"when you consider one in 100 people self harm then it's not unusual that in an ambulance service with 600 paramedics that, you know--, or whatever it is, then a lot of us are going to have family members who consider it, it could even be one of us working here" (Para 4).

Many of the paramedics reported accounts of patients whom they knew or had dealings with who had died by suicide following SH. The following case reveals the tragic outcome of a patient who had self-harmed on multiple occasions, and the paramedics perceived the system they worked within was sub-optimal in responding to the needs of the individual. The person involved appeared to be known to the paramedic community in the study area, and they encountered her for SH several times in the lead up to her subsequent death, where she left ED and was hit by a truck on a motorway. Paramedic 2 recalled how:

" if you look recently in the papers a known mental health patient that we've done, self harmer, left an ED, walked onto the motorway and was hit by a truck. I don't know if you came across that. She was a regular, I'd been out to her and been out to her a few times in the past.... about three or four times 
in total myself. So if you look at it for one paramedic only threelfour times there's multiples along the way....(Para 2)

Paramedic 2 reported how as he had attended this person many times in the past; he was aware of the patient's background and reflected on how these wider social and medical problems impacted on her frequent $\mathrm{SH}$ :

"There was longstanding issues in regards to her childhood whether there was abuse I'm not too sure 'cause we couldn't really get through to her. So normally it manifested itself--, one example you had, er, self harming in regards to she had a prolapsed rectum and she kind of--, she was cutting away at that and we used to take her in. She was an epileptic as well, so a lot of the time we take her in for the genuine medical reason of epilepsy but there was still the--, most of the time it was the underlying mental health issue that was there". (Para 2)

Paramedic 2 provided comment on the busyness of the emergency care system at that time, and how a busy ED may not have been the best place for her:

"she was taken in and again, as I stated just now, the system was full to capacity and she was put to one side and she walked and she walked--, 'cause the hospital is right next to--, right next to the motorway, within about 20/30 minutes walking distance, she had got onto the motorway and a lorry knocked her down.... it was about three or four o'clock in the morning.... She didn't have the optimal care, but I'm not blaming the ED staff for that--, Like I was saying before, if she does self harming and she's got a history of self harming and you know the best place to take her is a mental health facility for assessment, then that's where I should take her, not to a busy A\&E unit.... Soon after that there was another regular who did exactly the same and he had to be prevented from jumping off a motorway bridge." (Para 2).

Both Paramedic 2 appeared sad and angry when recalling this story. There was a sense of melancholy, and frustration around the system they worked within, which did not appear to be set up to provide optimal care for people who SH. Paramedics again reported that the ED was not always the appropriate care setting for people who SH:

"I don't believe that these are the place (ED) to take these people, but I have no other option. .... Because it's such a chaotic environment with people who are so hard pushed to deal with the patients coming in" (Para 7)

Despite this, paramedics reported limited other options other than the ED for people who SH: People who SH often require assessment and treatment for physical and medical problems in the ED following $\mathrm{SH}$. 
"Erm, the biggest difficulty is that we have two options. And the two options are, poisons unit for an overdose, but they won't take anybody with trauma, so if you've overdosed and you've cut yourself, they can't go there. Take them to the trauma unit, their injuries are treated, maybe they get to speak to the mental health nurse, maybe they get to speak to the psychiatrist, then they go home." (Para 9)

Given such limited options, Paramedics reconciled themselves to the notion that ED was their only option, and their job was to get them there, as explained by Paramedic 11:

"All I have to do is the three Ts. I have to triage you, treat you as appropriate, and transport you. And that's what I will do because there is no other option" (Para 11)

\section{Category 4: Managing Risk}

Paramedics expressed concerns about the need to manage risks for patients and themselves. They were concerned over how they would be judged if something went wrong, and worried about risks of complaints against them, litigation and losing their professional status. These perceived risks influenced paramedics' decision-making to ensure that patients who SH attend hospital, by force if necessary. Paramedic 2 has illustrated these risks by telling how:

"a lot of paramedics at this moment in time are extremely fearful of the way that they can be perceived, their communication skills. And they're fearful of complaints and litigation against them, and fearful of losing their professional status” (Para 2)

They were particularly concerned about the risk of a patient going on to die by suicide.

"if you leave somebody who has attempted to self harm at home, erm, that the chances are, and it is normally done on an individual basis, but the chances are that if you leave them there without proper assessment and proper mental health assessment, then the chances are that they might well continue to attempt to cause themselves self harm, and they might well succeed in ultimately taking their own life." (Para 11)

Paramedics showed great concern for this scenario occurring, and sadness if any such patient died by suicide following their care. They reported how they wanted to do what was best for patients following $\mathrm{SH}$, but they did also report heightened anxiety 
over the risk to themselves in this scenario. They were concerned that they would be blamed in some way, and they would get in trouble. Paramedic 1stated:

"I have the desires to do what's right--, what's best for the patients, but I don't see that in every paramedic because they've got this ulterior motive is bugger the patients, what have I got to do to stay out of trouble?" (Para 1)

Paramedics reported general anxiety and concerns about losing their professional status, their job, and their income as the result of a complaint following the death of a patient who had self-harmed and not been conveyed to hospital. Paramedic 2 explained that:

"they're fearful of complaints and litigation against them, and fearful of losing their professional status" (Para 2)

And

My concern is, is that if we do not assess and treat these patients correctly, and take them to a hospital, and something happens later then there's, obviously, concern about my practice. (Para 7)

Paramedics were concerned about getting in trouble for breaching legislation. They felt they were in the difficult position of having to reconcile their own concerns for breaching any such legislation against how they felt the patient may wish to receive care. Paramedic 2 shared such concerns, stating that:

"I am aware of the law and I don't want to fall foul of the law but I also want to do what I want to do and I don't want to let the patients down" (Para 2)

All of the paramedics reported this tension of wanting to do the best for the patients, but their ultimate goal was to stay out of trouble themselves, as articulated by Paramedic 7:

"paramedics are doing what they do to keep themselves out of trouble as opposed to help the patients" (Para 7)

Strategies employed by paramedics which aimed at ensuring a patient was not left at home, at risk of dying by suicide, are discussed later in this chapter. However, at the centre of this thinking around risk was sense self-preservation, a sense of 'not on my watch', as told by Paramedic 1:

"I mean if he wants to kill himself then I can't stop him doing that but I don't particularly want him to kill himself when I'm involved." (Para 1) 
They were unsure if this approach was the correct way of managing people who $\mathrm{SH}$, and reported how they could be criticised either way. Paramedic 6 said:

"So I mean, it depends on which way you look at it, I would say you're damned if you do, damned if you don't." (Para 6)

Paramedics were also concerned of the risk of aggression or violence from patients who SH. They were concerned for the safety of themselves, the patient and others. They acknowledged how the scene where somebody has self-harmed could be a threatening and dangerous environment, sometimes involving intoxicated patients, and implements used to $\mathrm{SH}$ which could be used as weapons against them and others. The paramedics described examples of SH taking place during patient contact, which was upsetting and traumatic:

"she smashed a bottle and stuck it into her groin, and was cutting her groin with the bottle, and it was quite, quite, quite nasty. She really bled out, so it was quite difficult to deal with." (Para 9)

When faced with aggression, violence and potential assault with weapons, paramedics reported feeling concerned and vulnerable. Paramedic 3 told of one patient:

"who'd committed self harm using a kitchen knife and had done a reasonable job. And when you walk through the door and she is still holding the kitchen knife, and she says, you know, "I don't want to live, you're not going to save me," and she waves the knife at you then, obviously, there's a little bit of feeling of self preservation, and turning around and walking out the door" (Para 3)

In such situations paramedics in our study valued assistance from the police. Sometimes police were used in the detainment of a person who had self-harmed, but in most cases they were there to protect the safety of the ambulance staff, the patient and others. If they were not already deployed to the scene, they would often be called, as told by Paramedic 8:

"I looked down and the patient had rolled over and she had a knife in her hand, but in like a dagger type action.... So you know, I obviously, put myself directly in danger there, I had no way of getting out of that room... I was basically trapped... she was between the door and me. So we backed off, erm, we had to ring the police from the house phone to back us up... the patient was fine, we ended up taking her into hospital" (Para 8) 


\section{Category 5: Confidence and Competence at boundary of mental and physical health needs}

Paramedics are used to assessing, prioritising, treating and referring patients within their care across a range of presentations. This often involves assessment, immediate care decisions and treatment options which may be needed to save life in the immediate or short term. However, they reported a lack of confidence and competence in such decision making in $\mathrm{SH}$, which straddles the boundary of mental and physical health needs. Unlike in physical or medical presentations, paramedics in our study revealed challenges over appropriate assessment, risk stratification, and referral. There were wide ranging views on the status of $\mathrm{SH}$ as a mental health problem. Paramedic 8 questioned:

"So you know, do these people have mental health issues or are they absolutely, as was pointed out to me on one occasion, carrying out a lifestyle choice?" (Para 8)

Differing views were put forward on whether SH was indeed a mental health problem. Paramedic 9 felt people who SH were suffering mental illness:

"In my view people who self harm, I'm generalising, there's still going to be exceptions, but they have mental illness" (Para 9)

Paramedic 1 suggested that:

"They're not mad. They have real issues" (Para 1)

Whilst Paramedic 7 provided an empathetic view, which echoes the RCPsych (2010) definition:

"you see some that self harm and you know that they are at their lowest ebb, and the self harm is a real either a last ditch call for help, or it is an absolute desire by them to take their own life to alleviate them from whatever situation or mental health problems they have. And I feel very, very sorry for them, you know" (Para 7)

The disparate views from paramedics interviewed reflected a lack of a shared understanding on whether SH was a mental health problem. Paramedic 9 appeared to be looking for further education and guidance, rather than trusting her own judgement, highlighting how she had never been informed whether or not $\mathrm{SH}$ was a mental health issue: 
"nobody's ever actually pointed out whether somebody who slits their own wrist has a mental health issue....(Para 9)

Paramedics in our study struggled with differentiating between physical, medical conditions versus non-physical mental health presentations, and the presence of both. Paramedic 2 told how with mental health problems:

“There's no wound." (Para 2)

Whilst Paramedic 11 explained that in SH:

"there's nothing to treat, you know. You're trying to treat something that's not there, like you know. There's no wound, there's no medical condition that you have, you know, you've got no tools to sort of, you know. So apart from the, you know, them actually self harming, and you know, which tends to be minor stuff, you don't get, you know, you don't get many sort of like serious wounds." (Para 11)

While Paramedic 4 highlighted the challenge with people who regularly SH and present to them, who may be suffering from a medical condition which could be overlooked:

"Even a regular patient that you come across, you know, so you could actually miss a medical condition." (Para 4)

Paramedic participants reflected on the need for caution when caring for patients who may be confused and self-harming, where such behaviour may appear to be from a mental health related cause, when in fact it is a symptom of a physical or medical complaint. Such situations may therefore require robust investigation either prior to, or in parallel with, mental health assessment. An example shared by Paramedic 2 was that of missing the diagnosis of a diabetic patient with a high or low blood sugar who can present as confused, agitated and may even be harming themselves because of their conditions. Paramedic 2 explained that the low blood sugar may be missed:

"Missing something like you know. Yeah, you know, because like you know, as you know like someone with a low or high blood sugar could, you know, could present" (Para 2)

The basis of such confusion was due to a lack of understanding and confidence in caring for people who SH. Study paramedics pointed out that their training and education focussed on technical and physical conditions such as trauma and cardiac 
presentations at the expense of mental health related issues. They suggested that greater understanding was needed to prepare and guide them to care for people who SH or have mental health problems, and that training should focus on:

"how do we treat that sort of mental health issue rather than the physical injuries 'cause I feel that we're not quite appropriately trained to do that, but more so how can we help them mentally rather than physically" (Para 8)

Paramedic 2 further expressed this concern regarding their confidence and training, and highlighted the potential for paramedics to play a role in providing emergency mental health care if given the appropriate training:

"what you would have liked to have done is either have the training and confidence to be able to provide emergency mental health care to this person" (Para 2)

Paramedics recognised how they could become frustrated at such self-destructive behaviours and how they sometimes displayed a lack of empathy towards such patients, but recognised the importance of gaining understanding of why people $\mathrm{SH}$, as described by Paramedic 11:

“Well, the whole mental health issue. The 'whys' the 'hows'. Having a comprehension of what goes on in that person's head to make them want to cut themselves, to make them want to put a rope around their neck. To make them want to take every tablet they can get their hands on. I think we need to understand the 'why' behind that." (Para 11)

Paramedic 6 recognised that in the absence of adequate training, confidence and competence, the care that they provided was not as good as it could be. When asked 'what do you think of the care that you give to people who SH?' Paramedic 6 said:

"Crap. It's pretty poor... We don't provide anything very much for them. We ask them a whole pile of questions about when they took the tablets, what they took them with, have they been sick, have they done it before. "What was your intention when you took those tablets?" You know, that's--, it's--, that's the bones, yes we have to do that. A lot of times that's all they get" (Para 6)

\section{Category 6: Professional, legal and ethical tensions}

Complex tensions were revealed by paramedics in applying challenging ethical and legal principles in clinical and professional practice. All study paramedics involved reported an understanding of the law in relation to $\mathrm{SH}$, but also reported lacking 
confidence in its application, as seen in the following quotation where Paramedic 5 said:

"I don't know if I feel confident in the law" (Para 5)

The paramedics were concerned over the risks involved in caring for people who SH and how they would be viewed if something went wrong, potential litigation, and the potential for loss of professional status or employment. Paramedic 2 explained:

"A lot of paramedics ... are extremely fearful of the way that they can be perceived...they're fearful of complaints and litigation against them, and fearful of losing their professional status." (Para 2)

This assumption resulted in them taking on a role as preserver of life, setting up a tension between acting in the best interest of the patient, and responding to the patient's wishes. Paramedic 4 explained the lengths they would go to in limiting risk:

"I've got to say, whether it's right or wrong, if I believe, even half believe that a person had taken an amount of medication that would seriously damage their health, and they were refusing to go in then I would think, and this is selfishly, I would think well, I'd rather get into trouble ethically for taking somebody against their will than somebody die, and I left them there to die. So then I think I would use whatever means I had to force them to a place of care." (Para 4)

Paramedics reported incidents where patients were lured outside the home where police could enact their powers under Section 136 of the Mental Health Act (MHA 1983); indicated here by Paramedic 6:

"If you wanted somebody detained then we all know that a detention under Section 135, and 136 needs to take place in a public place. Therefore often the easiest way to do it, to prevent somebody, or to treat somebody with selfharm was normally to try to coax them into a public place where a police officer could take action, because they were, obviously, very reluctant to do it in a private property. And yeah, I can think of several occasions over the years where you've said, "Just come out to the back of the ambulance, we'll just run some checks on you and you'll be all right, " and as soon as they've put their foot on the pavement then, you know, that's it." (Para 6).

The previous quotes from Paramedic 4 and 6 highlight the challenges they face managing a patient they believe to be at risk of suicide following $\mathrm{SH}$. Both paramedics were open about the lengths they would go to in order to protect 
themselves and patients, which appeared to involve deceiving patients. Paramedic 4 recognised this may be deemed to be selfish, and may get him in trouble, but he was prepared to take such risks to ensure the patient attended hospital and would not die by suicide. The strategy told by Paramedic 6 of deceiving a patient who had selfharmed into moving into a public place where Section 136 (MHA 1983) could be applied was revealed independently by several of the paramedic participants:

"A cigarette, come outside for a cigarette. So you know, that's using, I don't know, entrapment or something. I don't know what the word is. But certainly to get him outside under false pretences, and then once he was outside the property he could be arrested by the police cause he wasn't in his own property. Now I haven't studied that law, it sounds a bit odd to me, but who knows." (Para 5).

This quote reflects the uncertainty, lack of knowledge and confidence that existed in all the study paramedics in applying mental health legislation to cases of SH they encountered in practice. The quote also conveys the lack of education the paramedic had received in this area.

Paramedic 7 provided details of the negotiations that take place trying to convince a person to attend hospital following SH and how such incidents they can be very time consuming:

"There was some attempt to get him-, there was some talk about trying to get him to go outside where the police could arrest him because he was no longer inside...But anyway, we ended up being there for about four hours." (Para 7).

One paramedic reflected on the shared understandings that paramedics had around this strategy for detaining patients following $\mathrm{SH}$, telling how it was:

"known as the Ways and Means Act, and that was the ways and means of getting somebody--, and you know, I'll quite happily put my hands up to, on tape and say that, you know, I've been in situations where somebody has deliberately self harmed and the police have found themselves powerless to do anything" (Para 2)

Paramedics were uneasy with this position and felt these strategies had emerged from the many challenging issues they encountered with people who $\mathrm{SH}$, as discussed throughout this thesis. Paramedics reported how for both the police and paramedics, patients who SH could be very time consuming, as explained by Paramedic 5: 
"It's frustrating because, erm, I have had situations where the police have taken patients into hospital, and you know, you chat to the police then and they're not happy 'cause sometimes they get tied up 'cause these patients end up sitting in hospital for hours on end." (Para 5)

And also Paramedic 7:

But anyway, we ended up being there for about four hours. (Para 7)

Paramedic 3 reflected how:

"I think the easier decision would have been to force this gentleman into hospital, certainly. I mean if you wanted to be lazy about it, you know, that would have been the easiest thing, you know, less time we've seen, less head work and things" (Para 3)

Paramedics were unhappy with the current situation and resorting to 'ways and means' of ensuring patients are transported to ED for care. They felt the situation they found themselves in had emerged because their training, competence and legislation did not support their decision making.

Paramedics in our study recognised their approach of deceiving patients may be wrong, and counter to professional standards of registered health professionals such as themselves. They also showed some awareness of the need to respect a patient's decision-making capacity as enshrined in MCA (2005) legislation, however, they justified this approach by taking the position of preservers of life, acting in the best interest of the patient, even if contrary to patient's wishes and the patient has capacity to make their decision. They knew this could potentially be breaching the right of patient in determining their own interests, but they felt the situations they faced compelled them to act in this paternalistic manner. Irrespective of the degree of $\mathrm{SH}$, paramedics would not leave a patient at home following $\mathrm{SH}$ for fear of them conducting further $\mathrm{SH}$ :

"I wouldn't leave a patient at home that's self harmed 'cause it's likely they're going to self harm again” (Para 4)

Irrespective of the degree of $\mathrm{SH}$, suicidal intent was frequently assumed, as articulated by Paramedic 9:

"I think the question of suicidal intent, as interpreted by myself as a paramedic, anybody who causes them self, self harm then, you know, we 
automatically assume, and perhaps wrongly on occasions, that the intent is always an attempt at suicide" (Para 9)

Overall, when it actually came to balancing considerations of ethics, the law, or professional practice, saving life was the first priority:

"Now the ethicacy of that doesn't sit well with me, but if the gentleman is going to die from this overdose then, you know, what's more important life or ethics?" (Para7)

Paramedics admitted that legislation in this area was vague and complex. Paramedic 8 suggested that such legislation in this context would be influenced by legal challenges, and is yet to be tested. Paramedic 8 said:

"with these decisions, they're very much of an opinion, and until they are tested in law it's very difficult to come with a straight conclusion” (Para 8)

These actions may also be influenced by the limited repertoire of decision making options because of a lack of training in this area. The quote above also highlights a legal tension where current UK mental health legislation (MHA 1983) does not support a satisfactory outcome for the paramedics involved. Many ethical tensions may also exist, such as the need to balance the autonomy of the patient to make their own decision, with a duty not to leave this person at risk of suicide, especially as they may be suffering from a mental condition or lack decision making capacity.

\section{Summary}

The results of the interviews with paramedics in this chapter arose from the coding process of taking concepts and thinking how they could be subsumed under the higher-level headings of categories. Some of these categories presented in this chapter echo findings of previous studies from the perspectives of patients and inhospital staff. These include negative attitudes, values, judgements from healthcare staff towards people who $\mathrm{SH}$, and the sense of frustration at the frequency and repetitive nature of $\mathrm{SH}$ reported in the present study were also revealed in the systematic review and metasynthesis (Rees et al 2014, 2015). Paramedics in our study reported a lack of confidence and competence in caring for people who SH, which again has been reported by other professional groups such as doctors and nurses working in emergency care, as revealed by the systematic review and 
metasynthesis (Rees et al 2014, 2015). The categories constructed in this study do however present aspects unique to paramedic care for people who SH. These include the context in which paramedic care is provided, and the paramedic's vulnerability to violence and aggression. The isolation and system failure paramedics report in caring for people who SH is again amplified when working in the pre hospital setting, often alone, with limited support. Likewise, the professional, legal and ethical tensions paramedics face are influenced by factors such as availability of medical staff to conduct mental health assessments and difficulties in assessing mental capacity in patients who may be intoxicated. Finally, how risks are managed by paramedics is unique to their practice and may include talking, coercion and detaining the patient and the involvement of the police. These issues, which are exclusive to paramedic caring for people who $\mathrm{SH}$, are yet to be reflected in the published literature and are described further in the following paragraphs.

Rich insights were revealed in this study on the unique context in which paramedics provide care and the potential negative impact that busyness and tiredness may have on their care for people who SH. Paramedics provide care for people who SH in public places and people's homes and multiple influences may impact on care in such contexts. These include vulnerabilities of paramedics, patients and others to acts of aggression and violence in scenarios of $\mathrm{SH}$, where people may be intoxicated with alcohol, and implements used in the SH act which may be used as weapons in aggression directed towards paramedics and others. The context in which SH is encountered may also result in patients feeling a sense of shame and embarrassment when care is provided in the presence of relatives and others.

The sense of isolation and the failures within the health system reported by paramedics in our study appear to be of great concern. Whilst much of this perceived isolation and system failure may be beyond the control of paramedics, these results highlight the need for the organisation to consider paramedic care for people who SH and provision of services for people who SH. Paramedics sought support in their decision making in relation to people who $\mathrm{SH}$, whose care was seen to be straddling the boundary of mental and physical health needs. Paramedics revealed challenges over appropriate assessment and management of risks, and referral for people who 
SH. Complex tensions were also revealed by paramedics in applying professional, ethical and legal principles in cases of SH. Scenarios were presented illuminating these tensions and the challenges posed, which involved many risks to patients and paramedics. The risk most acutely felt was that of a patient going on to die by suicide following care and therefore, irrespective of the severity of injury from $\mathrm{SH}$, suicidal intent was frequently assumed, resulting in the patient being taken to hospital, by force if necessary. The forcible detention of a patient following $\mathrm{SH}$ reported in this study acutely reflects many of the issues raised by paramedics around their lack of confidence in assessment, legislation and decision making when caring for people who SH. Paramedics have evolved ways and means of managing patients in such circumstances, which may be circumventing legislation.

In the following chapter the basic social process (BSP) around which all of the categories revolve in paramedics' perceptions of caring for people who $\mathrm{SH}$ is presented. Finally, theoretical constructions are further conceptualised in the form of the final Evolved Grounded Theory. 


\section{Chapter Seven - Final Grounded Theory}

\section{Basic Social Process (BSP)}

Decision making in a context of risk was identified as the BSP in paramedics' care for people who SH. Risk and decision making, alongside anxiety about fault finding and aggressive disciplinary action, are familiar and significant issues across paramedic work (Gunnarsson 2009, Blair 2011). Paramedics have to make decisions about what care to provide, what onward referrals to make, whether or not conveyance to hospital would be appropriate, and how to handle the situation if the patient's decision about care differs from the paramedic's. Such decision making by paramedics can be divided into system I and system II processes (Jensen 2010). System I processes involve reflexive subconscious thinking, employing mental short cuts to minimise effort (Evans 2008), but can be prone to error (Croskerry 2009). System II thinking is employed when decisions need purposeful contemplation and analytic thought. Due to its focus on life threatening emergencies, much of paramedic training emphasises system I decision making, and may use protocols to reduce the need for time-consuming analysis. The complexities and multiple influences of paramedic care and decision making in SH requires type II decision making, yet paramedics report a lack of competence and confidence in this area.

Paramedics reported how many patients refused care on scene. This scenario presents professional, legal and ethical tensions (Rees et al 2016), and again reflects the BSP of decision making in a context of risk. The response to such risk can be seen in paramedic reports of patients being lured outside the home for police to enact powers under MHA (1983), which reveals inappropriate application of mental health legislation. This approach may be due to their limited repertoire of care options and decision-making strategies. This study provides potential for understanding why such practices of police/paramedic detainment of people who SH have emerged.

Paramedics in the present study were aware that detaining may breach ethical and legal principles, but they reported a lack of training and confidence in decision making in this area. Paramedics were concerned about the risk of subsequent suicide of a patient in their care and pointed to sanctions that could be imposed on them following such an event, which included being held accountable in law, by their 
employer, or loss of professional registration; these issues were also raised elsewhere (Blair 2011).

It was reported that patients who SH and are intoxicated with alcohol can be confused or lack capacity; they may also be suffering a medical problem. A common phenomenon within mental health care in the ED is that of diagnostic overshadowing, where a focus on a person's mental health diagnosis overrides the consideration of their physical health needs (Jones et al 2008). Crosskerry et al (2000, 2013) attributes this phenomenon to the cognitive bias of "anchoring" (ie, basing a decision on early observations), and it can compromise patient safety (Crosskerry et al 2013). Clarke et al (2006) also found this phenomenon a positive finding in their study, as several participants demonstrated an awareness that physical complaints can have apparently psychiatric manifestations, requiring robust investigation either prior to or in parallel with psychiatric care. Risk prediction tools have been developed to support decision making in SH and suicide and are recommended for paramedic use (JRCALC 2016). However, Quinlivan et al (2017) found that_such scales performed no better, and sometimes worse, than clinician or patient ratings of risk, and recognised their limited clinical utility, leading to a waste in valuable resources. In line with guidelines (RCPsych 2010), Quinlivan et al (2017) advised that risk scales should not be used to determine patient management or to predict suicide and should therefore not be relied upon by paramedics.

Paramedic decision making is complex. Shaban et al (2004) examined judgment and decision-making of paramedics in relation to mental health and suggested that uncertainty and risk exist in human clinical judgment in all forms and disciplines of medicine. In the same study, Shaban et al (2004) gives the example of error as described by Reason (1990) being active or latent in nature. An active human error is often immediately known, involving unsafe acts. Conversely, latent errors result from organisational positions and decisions, where damaging consequences may lie dormant for some time, before becoming evident when local triggering factors overcome the organisation's defence mechanisms (Reason 1990). 
Shaban (2004) cites cases of SH and suicide following care where paramedics failed to conduct comprehensive mental health assessments as an example of 'active error' (Shaban et al 2004 p.6). This study challenges this notion, arguing that within such decisions in paramedic care for people who SH, latent errors lay dormant. Paramedics in this study reported lack of training, confidence, competence, support and guidance in the assessment and management of people who SH. The interpretation and application of legislation such as the MCA (2005) and MHA (1983) also appears to be challenging in paramedic care for people who SH.

Therefore, if a patient comes to harm, or dies by suicide following paramedic care for SH, rather than being viewed as an immediately known, 'active' human error, such situations may reflect the complex issues above, which are already known? Many of these are wider professional, educational, organisational, societal and legislative issues, which become highlighted following such deaths by suicide, and may therefore be considered as 'latent' errors, lying dormant awaiting to be addressed.

\section{Final Grounded Theory:}

\section{Wicked Complexity in Paramedics' care provided to people who Self-Harm: a Grounded Theory (GT)}

Rich insights have been gained into how paramedics perceive the care they provide to people who SH. The emergent categories enabled in-depth understandings of complex issues, which offers the potential for improving care for this group of patients. Paramedic care for people who SH presents a 'wicked complexity'. 'Wicked' issues were first described by Rittel and Webber (1973), drawing on the work of Thomas Kuhn, the American philosopher-scientist, who argued that normal science such as physics was tame-paradigmatic, with consistent ways of attacking problems as opposed to the interpretivist-constructivist nature of knowledge in EGTM. Rittel and Webber (1973) recognised how 'tame' problems can follow algorithmic processes (such as protocols in paramedic practice or suicide risk assessment tools) for arriving at solutions to problems. 'Tame' problems are manageable problems, they suggest, and have a proper focus, appropriate definitions, and relevant information related to them. 'Wicked' problems arrive with built-in complexities, making them doubly difficult to address. They have no known algorithms for solution and simply identifying the problem can turn into a major task. 
This study revealed how many aspects of paramedic care for people who SH make it a 'wicked' problem. For instance, the social and cultural context of SH behaviour in a system designed to save life, incomplete understanding of the true scale of SH, lack of support services, treatment decisions, legislative and ethical complexities, educational preparation, the impact of alcohol, isolation, and the setting are all exacerbating factors in this wicked problem. These issues are classified in this study as either: usual factors which present in paramedic care, factors heightened by SH or factors specific to $\mathrm{SH}$. Addressing these issues carries extensive economic, cultural and financial burdens and opportunities. This EGT reflects such wicked complexity in paramedic care for people who $\mathrm{SH}$, which is underpinned by the BSP: Decision making in a context of risk, and the following influencing factors:

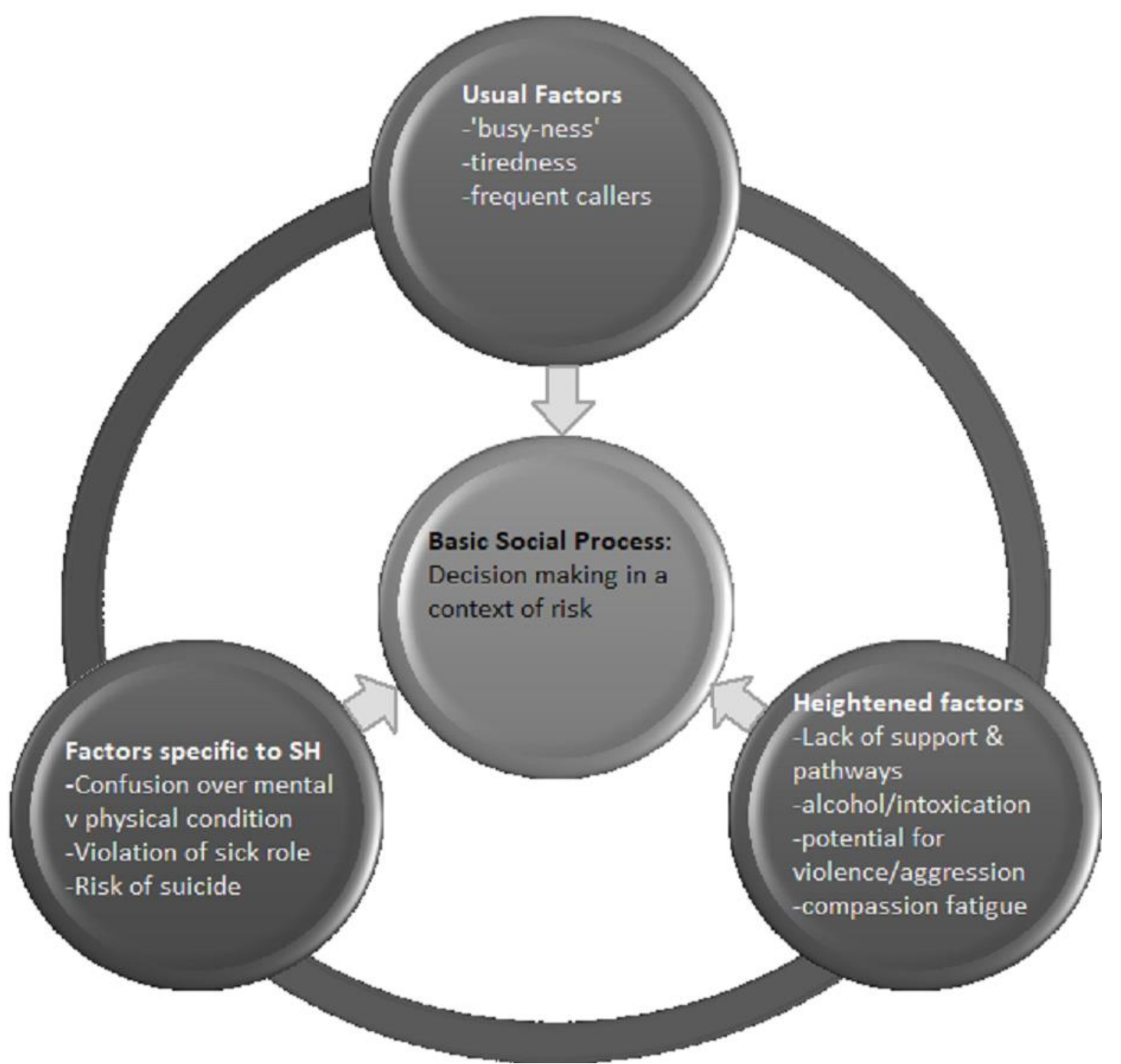

Fig 11. Overview of Grounded Theory (GT): Wicked complexity in paramedics' care for people who self-harm. 


\section{Usual Factors}

Many of the issues raised by paramedics were part of their routine practice, such as the impact of their tiredness or how busy they were. Such workload issues are not confined to $\mathrm{SH}$, as across the world demand for ambulance services has more than doubled in recent decades (Lowthian et al 2011, Christensen et al 2017). How they were feeling during their work was an important aspect in care for people who $\mathrm{SH}$, for instance they reported how being busy and not having meal breaks could negatively impact on their attitudes and the care they provide for people who SH. These findings are not exclusive to this study, nor paramedic work per se. The negative impact of busyness on attitudes towards SH was also reported by McAllister et al (2002a) and Suokas \& Lonnqvist (1989) who found that nurses in large busy hospitals were significantly less empathetic and had higher levels of negativity towards people who SH than those in non-acute settings.

Paramedics in this study reported frustrations at the frequency of attending people who $\mathrm{SH}$ and attending the same person on a regular basis is common in ambulance services (HSCIC 2015, WAST 2016, SG 2017). Robertson \& Henderson (2009) recognised the term 'frequent flyer' for such regular service users; this may be considered an offensive and pejorative term. The Frequent Callers Ambulance National Network (FreCANN) uses the term 'frequent caller' for a person who makes at least 5 calls per month or 12 calls over a 3month period. During 2014-15 London Ambulance Service received 1.7 million emergency calls; 1,622 individuals were 'frequent callers' generating 49,534 attendances, at a cost of $£ 4.4$ million (2015). Frequent callers are a high-risk group and more likely to die after their last visit to $\mathrm{ED}$ ( $2.6 \%$ of frequent users vs. $1.1 \%$ of infrequent users), become hospitalized at higher rates (18.8\% vs. $14.2 \%$ ), and be transported to the ED more frequently by ambulance (18.6\% vs. $12.1 \%$ ) (Fuda \& Immekus 2006). Frequent callers to ambulance services are more likely to be assigned call codes of substance misuse than any other condition, followed by psychiatric abnormal behaviour, and between $16-33 \%$ of frequent callers present as $\mathrm{SH}$ or suicide attempts (Pajonk et al 2001, Edwards et al 2015, Knowlton et al 2013, Brokaw 1998). Busyness and frequent callers are therefore common occurrences in paramedic care. It has also 
been previously acknowledged that a major role of EMS systems is to act as a safety net for persons with mental health problems (Knowlton et al 2013).

\section{Heightened factors}

Paramedics reported lack of support and being unable to access appropriate care from GPs, and Crisis Teams and alternatives to ED. However, alternatives to ED for patients attended by paramedics is becoming common and there is evidence that this is safe in areas such as falls (Snooks et al 2017). Risks have been highlighted in such ED avoidance in the general emergency care case mix (Morris et al 2017, O'Hara et al 2015). Paramedics in our study reported that such concerns and risks appear to be heightened in $\mathrm{SH}$, especially if alcohol was involved.

There is strong association between $\mathrm{SH}$, suicidal behaviour and alcohol (Murphy 2000) and approximately a third of patients reporting suicidal ideation have sought substance use disorder treatment two weeks prior to their suicidal ideation (Ilgen et al (2009). $\mathrm{Li}$ (2007) found $47.8 \%$ of SH injuries to be alcohol-related, and up to $46.1 \%$ of SH patients have consumed alcohol within six hours of SH (Haw et al 2005). The high incidence of alcohol and SH therefore suggests many patients may not be eligible for alternatives to ED.

Paramedics reported their vulnerability to acts of aggression and violence following $\mathrm{SH}$. Paramedics can be alone with patients or relatives making them easy prey to threats and acts of violence; indeed, between $61 \%$ and $90 \%$ of ambulance personnel have been subjected to physical violence during their duties (Pozzi 1998, Corbett et al 1998, Suserud et al. 2002, Boyle et al 2007, Rees \& Whitfield 2005) and 17\% have been threatened with a weapon (Suserud et al 2002). In SH such fears of violence are heighted amongst paramedics, as it was reported in this study that patients are often intoxicated, confused, lack mental capacity and weapons used to inflict $\mathrm{SH}$ such as knives and broken bottles are present and may be used on the paramedic. This concern of violence in SH may be justified, as Ilgen et al (2010) found that repetition of attempted suicide was associated with all forms of violence, with the authors concluding that individuals with histories of severe violence are at 
an elevated risk of harming themselves. Vaughan (2015) found that persons who SH are also at elevated risk for reporting abuse and violence toward others.

Paramedics indicated a lack of empathy in colleagues, and sometimes themselves, towards those who SH. Such lack of empathy towards distressed individuals has previously been reported in emergency care workers and may be attributed to compassion fatigue, which can occur when practitioners become emotionally exhausted and lose the ability to respond empathically to their patients (Gillespie and Melby 2003). Pejorative terms such as 'repeat offenders' were put forward by paramedics in the study. Whilst many of the paramedics displayed sympathy towards patients who SH using statements such as "I feel sorry for them", it has however been argued that with such sympathy, one can make a negative judgment about a person, followed by an "I" statement concerning what you are going to do about it for yourself. Empathy shared with patients is the building block of trust and rapport and the foundation for effective communication (Schindeler 2011). Unlike sympathy, empathy requires a perception of the immediate emotional state of the patient, and the second part is delivery or an acknowledgement of that perception to the patient; without both, there is no empathy (Graff 2013).

Such lack of empathy from paramedics towards people who SH may seem counter to their role as care givers, and self-destructive behaviour may challenge their function as preservers of life. A lack of empathy towards distressed individuals has previously been reported in emergency care workers, and may be attributed to compassion fatigue, which can occur when practitioners become emotionally exhausted and lose the ability to respond empathically to their patients (Gillespie and Melby 2003). Compassion is recognising one's own or another's distress, and attempting to alleviate it (Gilbert, 2009). Exposure to continual change, cutbacks, increased workloads and pressure to meet targets, augment pre-existing stress and are issues which have been attributed to compassion fatigue (Illes 2011, Beaumont et al 2015). Similar frustrations were revealed in the present study, such as the lack of facilities for appropriate care for $\mathrm{SH}$, tiredness and lack of meal breaks. Consequently, consideration should be given to these wider influences by policy makers, educationalists and leaders in healthcare. 


\section{Factors specific to $\mathrm{SH}$}

Many factors revealed in this study were deemed exclusive and specific to paramedic care for people who SH. Paramedics in our study reported confusion over whether a patient who deliberately harms themselves is suffering from a mental condition. The presence of a mental condition may influence the decision making, especially when detaining a patient under the MHA (1983). McManus et al (2009) found the rate of diagnosed mental illness in those who have died by suicide to be more than $80 \%$. People who SH and present to emergency carers also tend also to experience multiple tensions, both psychological and social in nature (Haw \& Hawton 2008, Hume \& Platt 2007). Such complexity has been found in ambulance service presentations of SH (Edwards et al 2015) and makes it challenging for services to respond to people who SH (Hunter 2003). Psychosocial assessment considers the inter-relationships between psychological and social domains of patients' lives (Hunter 2003), and it is therefore recommended that people who SH receive a psychosocial assessment before discharge (NICE 2004); despite this, only between $42 \%$ and $71 \%$ of SH presentations receive this assessment (Barr et al 2005; Kapur et al 2008).

Paramedic participants found difficulty understanding why people SH and they struggled to deal with the frequency of calls to the same person following SH. They reported lack of an observable medical condition in $\mathrm{SH}$ and frustration that "you've got no tools" as opposed to physical problems such as respiratory conditions where they had "tools". These views are present in other emergency care workers, and were articulated through the theme 'Frustration, futility and legitimacy of care' in the metasynthesis (Rees et al 2015). Nurok \& Henckes (2009) argued that in paramedic care a transaction can occur between judgments about the patient as a person and the technical dimension of enjoyable work, and that technical elements of physical care can take on a higher value than dealing empathetically with the patient. Nurok \& Henckes (2009) recognised how sociologists have long observed how personal patient attributes are highly valued and influence how patients traverse medical systems. They draw on the work of Timmermans (1999a, 1999b) on resuscitation in American EDs, which found that highly valued patients were treated as alive despite being biologically dead, and less valued patients were often treated as dead despite being biologically alive. Timmermans (1999a, 1999b) described this phenomenon as 
implicit social rationing and attributed it to judgments of social value. One may consider such circumstantial prioritization in the context of the present study; whilst people who $\mathrm{SH}$ are biologically alive, it would be an exaggeration to paraphrase Nurok \& Henckes (2009) interpretation of Timmermans (1999a, 1999b) that such patients may be dead to the attention of paramedic care. However, people who SH do appear to take on a lower priority for paramedics than those presenting with medical and physical symptoms, and there is a perception of them wasting time and resources for other more deserving calls.

Social value may also be influenced by the patient-paramedic relationship. In contemporary healthcare settings people become patients and in the sociological literature the concept of sick role has been used to outline the rights and obligations of patients. Talcott Parsons (1958 p. 176) proposed that health is "the state of optimum capacity of an individual for the effective performance of the roles and tasks for which he [sic] has been socialized". In the sick role, recovery depends upon a therapeutic process, and a patient is not responsible for their recovery but the therapeutic agent is. Another feature of the sick role is that the patient must try to get well, and in doing so, is obliged to seek help from competent agencies. Health professionals have certain rights also, which include invading the patient's privacy and instigating treatment. Frustrations from paramedic participants may result from patients not behaving as they should, and self-destructive behaviour being counter to paramedic work aimed at saving life. From the patient's perspective, many avoid care, often do not call for the ambulance and refuse ED attendance. Such avoidance of care violates Talcott Parsons' (1958) principle of the sick promoting their recovery.

Paramedics reported concerns over not conveying the person to the ED, and fear of harm coming to the patient should they be left at home. This is again an issue which spans emergency care as it has previously been suggested that $40 \%$ of attendances at EDs in the UK are avoidable (NHS-England 2013). Despite this there is lack of rigorous evidence on the safety and appropriateness of decisions to avoid ED by ambulance staff (O'Hara et al 2014). More recently Morris et al (2017) determined that $19.4 \%$ of all ED attendances may be avoidable, as ED provides opportunity for 
investigations and onward urgent referral. This may also be the case in $\mathrm{SH}$ as studies are yet to demonstrate safe alternatives to ED for patients cared for by paramedics. Concerns of harm coming to a patient who did not attend hospital are not specific to $\mathrm{SH}$; paramedics reported the need to protect themselves from challenges and this need to cover one's back was first reported by Porter et al (2007) and has since been recognised by others (Simpson et al 2017, Sharp et al 2016).

\section{Conclusion}

Patients who $\mathrm{SH}$ frequently present to paramedics who identify them as a particularly problematic patient group. Many of the challenges and risks in caring for people $\mathrm{SH}$ are present across the paramedics' case mix. However, there are heightened or specific to SH factors which make paramedic care for people who SH such a wicked problem. These factors include the role of alcohol and intoxication, and potential for aggression and violence, along with the challenges in assessing physical versus mental health problems and how they struggle to deal with $\mathrm{SH}$ because it is a violation of the sick role, running contrary to what they have been trained to deal with.

The built-in complexities of SH collide with paramedics' lack of education, confidence and competence in caring for people who $\mathrm{SH}$, along with lack of referral options and support. All this is being played out in the acute presentation of $\mathrm{SH}$, where significant harm or death by suicide are potential outcomes. The current situation presents the potential for latent errors for paramedics, with impact on their organisation and patients. Inappropriate detentions, negative attitudes and limited appropriate referral options may be adding to the significant numbers of people who SH that avoid care and are hidden to services that may be able to address their needs. Consideration should be given to improve training, referral options and support and to the possibility of extending current eligibility under the MHA to paramedics enabling them to transport people who $\mathrm{SH}$ and are assessed as a danger to themselves to places of safety for further clinical assessment. These may address some of the wicked complexity (tensions and challenges specific to $\mathrm{SH}$ ) of care by paramedics for those who SH. 


\section{Chapter Eight - Discussion}

\section{Introduction}

This chapter presents a summary of the main findings which considers how the aims and objectives of the study have been met, what the study has found and what it adds to our understanding of paramedic care for people who SH. This is followed by an in-depth discussion on how these findings relate to the existing literature across key areas.

The chapter then closes by considering the strengths and weaknesses of the study, the role of the paramedic researcher as an insider researching their own practice and the dissemination and potential impact of the study. Finally, the implications of the findings of this study on future care, policy and practice are presented along with recommendations and future research.

\section{Summary of the main findings}

The aim of this study was to explore paramedics' perceptions of caring for those who $\mathrm{SH}$ in order to inform education, policy and practice. This study has indeed produced rich insights into paramedics' perceptions of care for people who $\mathrm{SH}$, as presented in this thesis; a view which is yet to be reported in the literature. Attempts have been made by the author of this thesis to inform education, policy and practice, but substantiating such claims of influence on policy and education proves to be more challenging. It is however discussed later in this chapter, how, when considering the extent to which research such as this impacts on policy, the emphasis is on counting the potential occasions of influence, rather than trying to chart the unmeasurable or even unknowable outcomes of this influence (LSE 2011). These occasions of influence have included the results of this $\mathrm{PhD}$ being referred to or presented in peer reviewed publications, conference presentations, position statements and evidence to Government enquiries.

Whilst the metasynthesis and systematic reviews published from this study contributed to evolving our understandings in care for people who $\mathrm{SH}$, few studies included paramedics. By meeting the first objective in this study, this gap is however addressed, revealing paramedics' perceptions of caring for those who $\mathrm{SH}$, which 
were constructed into the following categories: Context; Judgements and Values; Isolation and System Failure; Managing Risk; Competence at the boundary of mental and physical health needs; Professional, Legal and Ethical Tensions. This is the first study to have explored such paramedics' perceptions of care for people who $\mathrm{SH}$.

The study has also met the second objective of developing a theory on paramedics' perceptions of caring for people who SH. A Grounded Theory was constructed reflecting the 'Wicked Complexity in Paramedics' care provided to people who Selfharm'. Within this grounded theory the Basic Social Process of 'Decision making in a context of risk' was argued to be the at the core of paramedics' perceptions of care for people who SH. This grounded theory is new knowledge, providing another perspective on the care for people who SH.

\section{What has this study found and what does it add to our understandings?}

\section{Key findings}

The following six categories emerged describing paramedics' perceptions of caring for people who SH:

- Context: This study reports how the context of paramedic care for people who $\mathrm{SH}$ is of importance, and that factors such as who called for the ambulance, the setting and the role of alcohol can impact on care.

- Judgements and Values: Paramedics report sophisticated and empathetic accounts with patients who regularly SH, however some of the judgements, values and language used may be as a result of a lack of understanding of SH. This may be dissuading people from accessing services, resulting in discriminatory care.

- Isolation and System Failure: A raft of policy and guidelines recommend support for paramedics in caring for people who SH and alternatives to ED. Despite this, paramedics interviewed told that this is not happening in practice, and how they feel isolated in their decision making.

- Managing Risk: Paramedics face many risks in the care of people who SH, risk of aggression and violence, risks to a patient who may refuse care and die 
by suicide which included professional risks, and risks of blame towards themselves should this happen.

- $\quad$ Confidence and Competence at boundary of mental and physical health needs: Paramedics reported they lack the confidence and competence in caring for people who SH. They recognised the challenge of distinguishing physical from mental health problems.

- $\quad$ Professional, Legal and Ethical Tensions: These were linked to limited decision support, referral options and education. Relationships with police revealed practices and surreptitious strategies aimed at overcoming complexities of care. These related to care and detention, which involved ways and means of ensuring patients attend the ED.

- This study suggests the Basic Social Process (BSP) in paramedic care for SH is that of Decision making in a context of risk.

- $\quad$ The final Evolved Grounded Theory that emerged was one of 'Wicked Complexity of paramedic care for people who $\mathrm{SH}$, which includes usual factors such as tiredness and frequent callers, heightened factors including lack of support and pathways, and factors specific to $\mathrm{SH}$ such as determining physical versus mental health problems, assessing mental health and suicide risk.

This is the first study to explore paramedic care for people who SH. A continuum of knowledge construction has evolved throughout this study, which has been published as four peer reviewed articles, constituting a significant body of knowledge on paramedic care for people who SH. This will be beneficial to clinicians, policy makers, legislators and others with an interest in improving care for people who SH.

\section{How do these findings relate to the existing literature?}

The systematic review and metasynthesis of the literature in this study revealed the paucity of published research exploring paramedic care for people who SH. It is therefore challenging to consider these findings within this context. However, in the following sections, the discussion considers the findings of this study in relation to wider relevant literature and in-hospital research which explores care for people who $\mathrm{SH}$ from the perspective of emergency care staff such as doctors and nurses. 


\section{Influence of the context of paramedic care for people who SH}

Paramedics interviewed in this study reported their frustrations of providing care for people who SH within a busy context. This was consistent with findings from inhospital studies which also report how such frustrations are amplified due to competing demands for care (Hopkins 2002). Challenges revealed in a study by Anderson et al (2003) included frustrations from in-hospital staff having to care for people who SH in full departments, alongside patients with conditions such as severe asthma or meningitis. However, competing demands for care are quite different in the paramedic context reported in this study. Paramedics in our study reported frustrations in terms of busyness and the loss of an ambulance available for the wider community for more urgent calls. These concerns have long been recognised, with claims that such misuse of emergency care places stress on services and may jeopardise patient care (Murphy 1998, Malone 1995). In a study exploring the nature of resilience in paramedic practice, Clompus et al (2016) revealed that mental health or alcohol related problems were deemed by paramedics to be social rather than medical problems, and patients presenting with such problems provoked a range of emotions, including anger that they were wasting the paramedics time and using resources they did not need. Negative attitudes towards SH from paramedics may therefore be influenced by the value judgements made by paramedics over the appropriateness and legitimacy of having to provide care for people who SH when their services are in great demand by people they perceive to be in greater need. Frustrations at such system pressures were again reported by paramedics in Clompus et al (2016), who directed some of their anger towards failures and gaps in the health and social care systems which resulted in such patients presenting to paramedics.

Paramedics in our study reported how busyness and tiredness in themselves could fuel frustrations and negative attitudes towards people who SH. Excessive workload, poor organization, conflicts, and the requirement to work night shifts are recognised chronic stressors in the work of paramedics (Oginska-Bulik \& Kaflik-Piero'g 2009). Clompus et al (2016) also argued that target driven policies, changes to skill mix, the increased pressures of workload, the introduction of performance management and resource constraints were having a detrimental impact on the paramedics' caring 
role. Clompus et al (2016) suggests a combination of these factors are increasingly affecting the paramedics' health and ability to cope, with tiredness and exhaustion being common experiences. Such busyness was deemed to be a 'usual factor' in paramedic work, and it appears that the busy working environments of emergency staff such as paramedics who care for people who SH, may be having a negative impact on the care for people who SH.

This study revealed other aspects of paramedic care for people who $\mathrm{SH}$ which were exclusively 'usual factors' for paramedics, due to the context of the environment they work in and how such patients present. Even prior to attending the scene, the events leading up to them attending people who SH were felt to influence the onscene care. Paramedics found themselves to be in an awkward position when relatives had called for the ambulance, as they perceived that the patient could be embarrassed and that relatives could get in the way of care. Benger \& Jones (2008) found that the strongest factor influencing direct ED attendance was the individual who summoned help; bystanders encountering a person with sudden illness in the community were more likely to dial 999, whereas patients and their relatives prefer to access primary care. Paramedics in our study however reported limited access to primary care, and it is argued this reflects the BSP of 'decision making in a context of risk' at work, even prior to the paramedics attending the patient. 'Decision making in a context of risk' may be a usual factor underpinning all emergency calls to the ambulance service, as studies suggest a confusion between the need for urgent medical assessment and the need for an ambulance response (Kirkby \& Roberts 2012), with patient and carer anxiety and decision making a common factor underpinning many emergency service calls (Booker et al 2014). One participant in Booker et al (2014) cited anxiety and feeling out of control as a reason for calling an ambulance and felt she needed urgent reassurance that her symptoms were not the sign of serious illness.

Such reassurance of paramedics arriving at the scene of an incident has also been reported by police. A police officer in Charman (2014) stated:

'it is a sense of relief when they turn up' (B8), 'everyone just breathes a sigh and of relief' $(\mathrm{P} 7)$, 
"You're trying your best at the scene and you are 'Where's the ambulance. Where's the ambulance.' Or 'When's the paramedic coming?' And they're there. And it's just a relief." (T2) (p115-116)

and in the case of mental health emergencies:

"So I think when you're sitting there holding on to someone that's falling to bits, and the paramedic turns up, friendly face, professional, 'Thanks. I'll take over now.' (p115-116)"

It would seem natural that paramedics are more informed than patients or the police to judge the severity of $\mathrm{SH}$, and paramedics therefore may play a role in stratifying risk for patients and family members, which is timely and in a manner which reassures patients.

Patients, police, bystanders and family members therefore need reassurance and support when faced with a person needing urgent care. Whilst health care professionals may become frustrated at this, one could argue that such advanced knowledge is a part of being a health care professional. This may be of greater significance with respect to paramedics as Booker et al (2014) found that the immediacy of provider response was a key factor in decision making when calling an ambulance, regardless of the eventual management or clinical outcome.

It was reported by paramedics that the presence of family members following $\mathrm{SH}$ could be problematic. There may be many reasons why family members can cause such difficulties for paramedics in caring for people in the aftermath of SH; they may be scared, emotionally upset or angry. Previous literature suggests that interpersonal relationship problems in the family context are important reasons for attempted suicides (Adityanjee 1986 Kattimani et al 2015, Kumar et al, 2013 Grover et al 2016)), and Ma \& Shek (2014) found that adolescents with more family conflicts were prone to having higher levels of SH and suicidal behaviours. It is therefore important to recognise there may also be contextual information that paramedics are not privy to, such as existing family conflict; indeed, the Royal College of Psychiatrists (2010) states that:

"The main triggers for self-harm are usually social, particularly family, issues, relationship break-up" (p 29)

Underlying the challenges of caring for people who SH lay multiple issues which are beyond the capacity of paramedics or ambulance services to resolve and which 
reflect the 'Wicked Complexity in Paramedics' care provided to people who SelfHarm'.

\section{The role of drugs and alcohol on care}

The role of drugs, alcohol and the environment in which they were consumed was perceived to have a negative influence on paramedic care for people who $\mathrm{SH}$, and this spanned many categories. Drugs and alcohol were perceived by paramedics in this study to play a role in limiting mental capacity, influencing application of legislation and safety. The role of alcohol intoxication is widely recognised to make up a significant proportion of the paramedic workload and has the greatest impact on frequent EMS use (Brokaw et al 1998), indeed 70\% of emergency department admissions at peak times are due to alcohol (WG 2015). Paramedics in this study also reported resistance from GPs and Crisis Resolution Teams (CRT) to accept a referral for a patient who has consumed alcohol, and alcohol consumption can indeed be an exception to CRT referral (National Institute for Mental Illness in England 2015). Paramedics in our study reported the strong association with $\mathrm{SH}$, suicidal behaviour and alcohol, which has also been reported in the literature (Murphy 2000 Ilgen et al 2009, Li 2007, Haw et al 2005). It follows that many of the patients who $\mathrm{SH}$ will have consumed alcohol and will therebefore be ineligible for CRT referral and result in ED attendance.

NICE (2004) recommend that temporary overnight admission may be needed following an act of $\mathrm{SH}$, especially for people for whom psychosocial assessment proves too difficult because of drug and/or alcohol intoxication. Our study paramedics report that $\mathrm{ED}$ is often their only option for people who $\mathrm{SH}$, therefore, until guidelines are amended and services improved, their current practice of ensuring patients attend ED may be the safest option for caring for people who $\mathrm{SH}$ and are intoxicated with alcohol.

The accounts of paramedics' vulnerability to acts of aggression and violence following SH adds to the 'wicked complexity' of paramedic care for this patient group, as reducing such aggression towards paramedics carries significant burdens and societal influences to solve. It has been highlighted that more than $75 \%$ of 
ambulance personnel have experienced threats and/or violence when performing their duties (Suserund et al. 2002). Maguire (2018) recently examined cases of serious claims of injury related to assaults and violence in Australian paramedics, finding that the total number of violence-related cases had increased from 5 to 40 per year, cases of injury secondary to assault tripled and the rate of cases by call volume had doubled. Maguire (2018) noted that although interventions had been attempted, violence against paramedics continues to be a growing problem, and effective solutions will likely be multifaceted and include training, engineering changes, community education and adjustments to agency policies. Maguire (2018) also recognised that due to the widespread nature of the risks with aggression directed at paramedics, empowerment of a national commission was needed to address this growing problem. Wider aims to understand and address the effects of drugs, alcohol misuse and aggression in society along with their effects on services may therefore positively impact on paramedic care for people who $\mathrm{SH}$.

\section{Competence with physical mental health care needs}

Paramedics in this study reported the challenges of determining whether the person presenting with SH was suffering from an underlying medical condition which they may miss, especially if they had consumed alcohol. This was deemed to be a 'heightened factor' in paramedic care for people who $\mathrm{SH}$, and was articulated in the theme 'Competence at the boundary of mental and physical health needs'. The phenomenon of diagnostic overshadowing was forwarded, where a focus on a person's mental health diagnosis overrides the consideration of physical health needs (Jones et al 2008). Diagnostic overshadowing is common in medicine; however, this study revealed many factors related to the skills and competence of paramedics in assessing patients who $\mathrm{SH}$, along with the challenging context in which the deliver this care, which are yet to be reported elsewhere.

Many examples were reported within the study of physical conditions that may mimic SH with confusion and potential for aggression; this included the presentation of a diabetic emergency. People with such underlying physical or medical conditions may also be at greater risk of SH, as patients with Type I Diabetes Myelitis have almost doubled risk of depression and suicidal thoughts compared to the general 
population (Rush et al 2008, Harjutsalo et al 2011, Westling et al 2004, FullerThomson \& Sawyer 2009), and are three to four times more likely to attempt suicide than the general population (Roy et al 2010).

\section{Influence of geographical setting of SH}

Paramedics in our study perceived there were poorer attitudes towards $\mathrm{SH}$ from paramedics working in an urban area than those in rural areas. They acknowledged that this may be due to urban paramedics experiencing more cases of $\mathrm{SH}$, which were more likely to be aggressive. Paramedics interviewed also believed that $\mathrm{SH}$ in a rural setting involved more lethal means than in an urban setting.

Some of these perceptions by paramedics of the geographical influence on rates and lethality of SH are also borne out in the literature, and in many countries, completed suicide rates are higher in rural than non-rural areas (Judd et al 2006, Hirsch 2006). This includes rural Australia (Caldwell, Jorm, \& Dear, 2004; Dudley et al, 1998), Austria (Kapusta et al, 2008), Scotland (Levin \& Leyland, 2005; Obafunwa \& Busuttil, 1994), and China (Yip, Callanan, \& Yuen, 2000). More recently, suicide rates amongst males in England were also reported as being highest in rural areas, even after controlling for social deprivation (Gartner et al 2008). Harris \& Hawton (2011) found that urban SH rates were substantially higher than rural rates and this relationship was sustained even when socio-economic deprivation and social fragmentation were accounted for. Rhodes et al (2008) also found that self-poisoning presentations by rural residents were more medically serious than their non-rural resident counterparts. An explanation for this difference has been suggested to lie in the increased likelihood of access to lethal means such as firearms amongst rural populations (Dudley et al. 1998). However, Harriss \& Hawton (2011) suggest that difficulty in accessing emergency medical services from remote and rural areas may also influence the likelihood of fatality.

Paramedics in the present study did not attempt to justify their perceptions of geography on the lethality of their SH case mix, however isolation and social fragmentation, which can occur in rural areas, may be more strongly associated with suicide than SH (Hemsptead 2006, Whitley et al 1999). Two hypotheses have been 
forwarded about the possible relationship between health and environment which may be pertinent to geographical variation in lethality of SH (Hawton \& Harrs 2011); these include the 'drift' and 'breeder' hypotheses. The environmental breeder hypothesis proposes that those from lower social classes who are living in urban areas are more at risk of developing mental ill health due to stress factors associated with living in inner cities, which includes stress resulting from high population density, high levels of unemployment, poor housing and low socioeconomic status. Exposure to such environmental factors, has a direct or indirect impact on an individual's health (Macintyre, Maciver, \& Soomans, 1993). Rural populations may face different problems particular to their region such as a lack of employment opportunities or affordable housing, inaccessible public and health services, or increased levels of social isolation. The drift hypothesis proposes that persons with certain risk factors for ill-health are more inclined to live in particular types of area, either by moving to, from, or staying put in certain environments (Verheij 1996). Paramedics, the ambulance services that employ them and care providers should therefore consider the influence of geography when organising services and care for people who $\mathrm{SH}$.

\section{Approach towards risk in the care of people who SH}

'Decision making in a context of risk' was identified as the BSP, and was deemed to be central to the GT, helping explain the social processes that influenced paramedics' perceptions of care for people who SH. Risk was also a 'heightened factor' in paramedic care for people who $\mathrm{SH}$, but not unique to paramedic practice. The triage of mental health presentations is universally described in the literature as complex and poorly understood (Clarke et al. 2007, Creaton et al. 2008, Gerdtz et al. 2009, Broadbent et al. 2010). Risk prediction tools have been recommended for paramedic use (JRCALC 2016), however it was acknowledged in this study that such scales performed no better than clinician or patient ratings of risk, leading to a waste in valuable resources, and should not be used to determine patient management or to predict suicide (RCPsych 2010, Quinlivan et al, 2014, 2017). Paramedics and other health professionals face the challenge that demographic risk factors increase the suicide risk of a whole population across its lifetime, but do not predict suicide in an individual at a single time-point, nor does the absence of such risk factors mean 
absence of risk of suicide (Cole-King et al. 2013). Predicting suicide is therefore challenging due to its rarity and risk factors co-occur commonly in people who $\mathrm{SH}$ (Kapur 2006). Risk factors for suicide in SH may also be common in paramedic work, as over $40 \%$ of ED patients have risk factors for suicide and $12 \%$ have past suicide attempts (Boudreaux et al 2006, Folse et al 2009); in one study 12\% of ED patients without chief complaints of mental health issues reported suicidal ideation, but this was detected by ED staff under $20 \%$ of the time (Claassen et al 2005). Paramedics therefore have a role in identifying risk factors, which may be common in their general case mix, however the challenge is determining what the appropriate course of action is when such factors are identified.

Whilst it has previously been argued that people who $\mathrm{SH}$ are at greater risk of dying by suicide than the general population (NICE 2004, MIND 2010), most people who SH will not progress on to die by suicide. Therefore, such decision making which focusses on prevention of suicide may be flawed as people who SH cannot usefully be categorised into those needing psychiatric care, or those who are at higher or lower risk of suicide. Hopkins (2002) reflects some of the anxieties that paramedic endure with such an approach to risk focussing on prevention of suicide, by suggesting that linking a quantifiable improvement in society's mental health with a reduction in the number of suicides places a heavy burden for mental health services to bear. Hopkins (2002) cites an article in the Independent on Sunday by Dr Robin Arnold, Chairman of the British Medical Association's Psychiatry Committee who stated:

"While physicians are accountable for what they do to a patient, psychiatrists are seen as responsible for what their patients do" (Dobson 1998),

This reflects concerns from paramedics in the present study of being held responsible for what a patient may do (such as dying by suicide) following their care. Therefore, given the limits of what is foreseeable in terms of an individual's likelihood of suicide, the current approach from paramedics to risk and the care of people who SH is devoid of clear answers on what is the correct course of action in such circumstance. Despite this, paramedics were not prepared to leave a person at home following $\mathrm{SH}$ due to their concern for suicide. 
Paramedics in our study recognised the rights of a person with mental capacity to determine their own treatment but, for some, the very act of SH represented a lack of capacity. This approach to risk would sometimes result in coercive actions and detaining people, sometimes using a range of 'ways and means', as revealed in the category 'A challenge to Legal, Ethical and Professional standards', where paramedics would rather get into trouble for taking somebody against their will, than leaving a patient who has self-harmed at home with the risk of them dying by suicide. Callaghan et al (2013) warns of such a position, maintaining that fear of such vulnerability is a dangerous concept, as it enables discredited and discriminatory approaches to capacity based on a person's status, specifically that a person with a mental illness may be judged both to be disabled and vulnerable and deprived of their decision making freedom on that basis, rather than on the basis of any consideration of their actual abilities. Callaghan et al (2013) also reflects the danger in the above approach of paramedic care for people who SH; that the notion that suicide is so repugnant that it should be considered outside the scope of autonomous decision making and that where persons are at high risk of suicide, coercive detention in hospital should be permitted where it is thought necessary to prevent that eventuality.

The approach to perceived risk by paramedics in the care of people who SH may therefore be empirically unsound? If suicide prevention is a clearly definable goal, one cannot usefully categorise people who are at higher or lower risk of suicide due to the limits of what we can know about an individual's likelihood of suicide. Callaghan et al (2013) argues that a public policy that allows involuntary preventative detention of competent persons thought to be at risk of suicide, places too great a burden on all persons living with mental illness to be justified. They also hold that policy makers must be realistic about the limits of the ability of medical practitioners to predict suicide, along with the magnitude of the impact on people who will not die by suicide if the presence of perceived risk factors alone is used at the basis for involuntary treatment, and of the true costs of detaining competent people who do not wish to have treatment for a psychiatric illness, in terms of loss of ordinary rights. 
Callaghan et al (2013) suggests that people who SH and retain decision making capacity should be able to refuse treatment, and that a perceived relative increase in suicide risk should not be used to override that refusal. This is not just because laws allow for competent non-psychiatrically ill people to make decisions not to have medical treatment even if there is a risk of harm or even death, but also because UK and international laws reflect that all people should be treated equally with fundamental rights to autonomy, and a private life (United Nations 2006, 2011). Rather than coercive treatment being triggered by an assessment of the likelihood of harm, a greater emphasis should be made on considering the patient's perspective, versus the negative experience of care being forced.

It was previously discussed that transport to ED was the safest option for paramedics caring for people who SH due to the risks that they present but that ED was also their only option. Paramedics favoured alternatives to ED and felt this was not the most appropriate place to care for people who SH. Therefore, achieving a state which respects the wishes of patients may require a range of alternatives to ED along with clarity around legislative issues, education and guidelines to support such decision making.

\section{Legislation}

The present study revealed 'Professional, Legal and Ethical Tensions', which influenced construction of the BSP of 'decision making in a context of risk'. Paramedics reported the challenges they faced with applying legislation such as the MHA (1983) and MCA (2005) in caring for people who SH, especially in those who refused care. The legislative challenges revealed in this study are reflected in wider literature and have been described and published by the author of this thesis (Rees et al 2014, 2015, 2017, 2018). These issues have also been discussed within the theme 'Decision making in self-harm: balancing legislation, risk and autonomy'. Applying legislation has been reported to negatively influence attitudes of nurses towards $\mathrm{SH}$ (McAllister et al 2002), who identified four dimensions explaining variations in attitudes towards people who SH which included perceived confidence in assessment and referral, ability to deal with clients, legal and hospital regulations guiding practice, and an empathic approach. Paramedics in Australia have also reported 
similar challenges with legislation within their wider care for people presenting with mental health problems (Shaban 2005).

The study reported how relationships between police and paramedics had evolved in the care of people who $\mathrm{SH}$, where practices and surreptitious strategies related to care and detention had emerged that were aimed at overcoming complexities of care. These strategies were termed 'Ways and means', and understanding conditions which have led to the evolution of 'Professional, Legal and Ethical Tensions' and use of 'Ways and means' is vital to improving paramedic care for people who SH.

Whilst paramedics in our study were not confident in MCA (2005), they reported the need for a mental capacity assessment and provided many accurate examples of its application, which reflected principles of SDM. However, when a patient refused care, paramedics in our study were less clear; SDM and MCA (2005) principles in this scenario came second to the risk of the patient dying by suicide. Mental health legislation such as MCA (2005) and MHA (1983) should comply with international human rights laws (United Nations 2011). The Convention on the Rights of Persons with Disabilities (United Nations 2014) enshrines into international law the human right that individuals with mental disorders must be granted legal capacity on an equal basis with others in all aspects of life, and upholds an individual's right to be free from involuntary detention and not to be forced to undergo mental health treatment (United Nations 2014, United Nations 2006). This international law is of importance to paramedic care for people who SH and MHA (1983) and MCA (2005).

Laws such as the MHA (1983) which allow involuntary treatment and detention in an emergency based on the risk of harm to self and others are widely justified (Røtvold \& Wynn 2015, Mavrogiorgou, Brüne, \& Juckel, 2011). Such emergency powers involve police and account for a considerable proportion of individuals receiving involuntary treatment (Burns, Jhazbhay, \& Emsley, 2011). However, reports of the 'ways and means' paramedics and police use to detain people following SH reflect wider concerns with laws which rely on urgency and risk of harm to justify involuntary admission and treatment and the protection of human rights of people 
with mental disorders (Wickremsinhe et al 2018). Reports in this study, and others (Rees et al 2016, IPCC 2015), of surreptitious strategies with police and paramedics involving manipulating individuals out of their home into a public place where they can be detained under Section 136 of the MHA (1983) are troubling, and may not be that uncommon.

Our paramedics queried how they would fare with legal challenges to such strategies. Brown (2014) presents three examples where there have been challenges to such strategies: in Webley v St George (2014) a person experiencing suicidal ideas was detained for affray by the police, as they could not be detained under Section 136 (MHA 1983). In Sessay v South London and Maudsley NHS Foundation Trust (2011) police argued they had applied the MCA (2005) when removing a person they perceived to be at risk from a private dwelling, but the courts ruled their actions were unlawful. Whilst in Seal v Chief Constable of South Wales Police (2007) a person was detained in the home to prevent a breach of the peace then, when outside, they were detained under Section 136 of the MHA (a similar situation found in our study); this was again ruled to be unlawful. The England and Wales Independent Police Complaints Commission (IPCC 2015) have also found examples of officers carrying out unlawful Section 136 detentions by detaining people in private premises; interviewees talked about individuals who had been 'enticed' outside then detained under Section 136 (a similar scenario to that reported in the present study). It was stated in IPCC (2015) that this was generally done because officers were either concerned about the welfare of the individual or did not feel they had time to wait for a warrant to be obtained under Section 135 of the MHA (1983) (to lawfully detain someone in a private premise), and did not feel they had any alternative options for detaining the individual. Such concerns appear to be driving the circumvention of legislation identified in this study in the name of urgency and risk, suggesting the legislation is either poorly understood and possibly not fit for purpose.

Legal challenges to the detainment of people who SH and refuse care are complex and sometimes conflicting. In relation to suicide, the courts have recognised a State interest in the prevention of suicide ( $\operatorname{Re} \mathrm{T} 1993$ ), yet any measures taken to prevent suicide and SH must be viewed in the context of the European Convention on 
Human Rights (ECHR 1950). Article 2 of ECHR (1950) confers a positive obligation to take appropriate measures to safeguard life which should not justify extreme or disproportionate measures of control intended to deprive the individual of any opportunity to SH. Keenan v UK (2001) set out the principle that authorities must discharge their duties [to protect Article 2 rights] in a manner compatible with the rights and freedoms of the individual concerned, reflecting the principle of proportionality, which calls for measures which interfere with personal autonomy, the right to respect for private life and physical integrity, protected under Articles 5 and 8 of ECHR (1950), to be limited to those who are required to be protected from $\mathrm{SH}$, and appropriate to the particular circumstances.

Paramedics in our study recognised the duty of care they and the police owed to patients and others, and their obligation to act in the patient's best interests when capacity was lacking. Breaches of the duty of care can result in claims of negligence, the defence of which derives from Bolam v Friern Hospital Management Committee (1957) which recognised there could be differences of opinion regarding proper medical treatment. More recently the case of Savage v South Essex Partnership (2008) involved a woman with a history of mental illness detained under the MHA (1983) who was given leave to return home from hospital and later died by suicide. In this case UK courts extended to the hospital trust the ECHR (1950) obligation to protect the lives of detainees with mental health problems. Rabone and another $\mathrm{v}$ Pennine Care NHS Foundation Trust (2012) is another similar case which established the test of the individual clinician's responsibility to mitigate real and immediate risk. In this case a female who attempted suicide and was voluntarily admitted into hospital several times, eventually allowed home by the psychiatrist and later died by suicide; the family successfully argued that the decision to discharge was negligent. Lord Dyson described 'real' risk as a 'substantial or significant risk and not a remote or fanciful one' (paragraph 38). The risk does not need to be imminent, rather it needs only to be 'present and continuing' (paragraph 39). This suggests the need for foresight and the undertaking of a proper risk assessment, yet no such tools exist which reliably predict risk of suicide (Quinlivan et al 2014, 2017). However, in Rabone, the courts also recognised there was no formal mental capacity assessment made on the patient, as she might well have lacked the capacity to make an 
autonomous decision to take her own life (paragraph 105). A formal assessment of capacity is therefore key to the care of people who $\mathrm{SH}$, together with risk assessment, and many of our study paramedics did indeed recognise the need to carry out such assessment of mental capacity.

Both cases above echo concerns in the present study from paramedics around risk of suicide. Despite such rulings, these situations remain complex from the paramedic perspective. Paramedics in the present study report having limited support and referral pathways for SH, and lack of access to medical staff and AMHPs with legal powers of detention following SH under the MHA (1983). Medical personnel and AMHPs were reported to be rarely available during encounters with $\mathrm{SH}$, resulting in the use of police powers such as Section 136 (MHA1983). Such use of mental health legislation is recognised as being based on urgency and risk of harm (Burns, Jhazbhay, \& Emsley 2011). However police are not registered health professionals and therefore may rely on limited interpretation of what constitutes a mental disorder. Such non-clinical characterisation of an individual by subjective judgment of traits, or behaviours that are stereotypically presumed to apply to individuals with mental disorders can be vulnerable to human rights abuses (Fistein et al 2009). Indeed, Wickremsinhe (2018) found non-existent or person-description definitions of mental disorder in $39 \%$ of mental health laws analysed, and argued that such laws may endorse human rights violations by permitting treatment without adequate assessment of disorder. The England and Wales mental health legislation (MHA 1983) may therefore need amending to reflect the situations in which paramedics find themselves.

The Australian Mental Health Act (2000) Section 33-35 (p.47-49) authorises police officers and ambulance officers to make emergency examination orders and detain patients with mental illness and imminent risk of significant physical harm to the person or someone else. Despite some paramedics reporting confusion over its enactment (Shaban et al 2005), such legislation is reflective of the current UK context of police and paramedics managing SH patients in crisis with limited access to medical professionals. As paramedics are often the only registered health professional in attendance following $\mathrm{SH}$, it may therefore be appropriate for 
legislation, mental health policy, education and practices to be amended to reflect the vital role of paramedics providing medical and mental health assessments to people who $\mathrm{SH}$, along with their ability to refer to a range of services.

\section{Social value and stigma in paramedic care for people who $\mathrm{SH}$}

A minority of paramedics interviewed were open and frank with their attitudes towards people who SH, telling how they were a "nuisance" (Para 3 \& Para 4), with one paramedic using the pejorative term of "self harm regular repeat offenders" (Para 6). All of the paramedics did however acknowledge that such attitudes were very common amongst their paramedic colleagues, with the following terms being used: "pull herself together, sort herself out, stop wasting our time" (Para 1). Paramedics in our study did however distance themselves from such negative attitudes.

Paramedics reported difficulty in understanding why people $\mathrm{SH}$ and revealed how it challenged their role as care givers and preservers of life. SH was deemed to be at odds with 'sick role' as set out by Talcott Parsons (1958), which outlines a shared societal understanding that those who are sick are not responsible for their status but do have a responsibility to try to get better.

This disjunction between the deliberate nature of $\mathrm{SH}$ in violating the sick role versus other areas of practice and non-deliberate presentations to paramedics may lead to them making value judgements about people who SH (Nurok \& Henckes 2009), regardless of clinical need. Nurok \& Henckes (2009) reported that, in the US, emergency services are a means by which large numbers of uninsured patients, or those who cannot transport themselves to a hospital, access care and are often used as a taxi service. Nurok \& Henckes (2009) captured such a situation in observations of paramedics at work, describing how she was:

"Waiting with a New York paramedic team. We receive a call for a patient living in a housing project known by the team to have many patients with HIV/AIDS. The team complains about being given another lousy case and starts driving to the call without much conviction." (p.506)

Negative attitudes towards people who SH was earlier in the study linked to the frequency of encounters. Suokas \& Lonnqvist (2009) reported that 76\% of ED staff 
agreed that patients who attempted suicide wasted staff time, $28 \%$ of staff agreed these patients misuse treatment facilities, and $51 \%$ of staff agreed that these patients take up staff time that is sorely needed by others in greater need of help. The metasynthesis reflected this situation through the theme 'Frustration, futility and legitimacy of care', the risky behaviour of SH being viewed as a potential waste of life by paramedics. There was also a distinction made between legitimate and illegitimate needs for care, with medical cases taking on a higher priority than those who SH.

There is an abundance of evidence suggesting people who SH encounter such negative and unhelpful attitudes from health professionals (Mental Health Foundation 2006). Negative attitudes towards people who SH were also reported in the systematic review and metasynthesis conducted within this study (Rees et al 2014, 2016). Despite this, stigma and negative attitudes towards SH can vary between healthcare professions (Mackay and Barrowclough 2005, Warm et al 2002). Whilst the findings of stigma and negative attitudes reported in the present study are not new, it is important to acknowledge and address such stigmatising attitudes towards SH from paramedics, as they can impact negatively upon the patients' psychological development and well-being, along with being a barrier to treatment seeking, adherence and efficacy (Link and Phelan 2006, Penn and Wykes 2003).

Paramedics in this study revealed many empathetic and caring accounts, often with individuals with whom they had developed a relationship over repeated encounters following SH. These accounts were complex and were given in a context which is yet to be reflected in the literature. Patients who regularly SH were often known to paramedics and their colleagues, and many scenarios were referred to, from minor cutting to serious injuries and disturbing scenarios of $\mathrm{SH}$. Paramedics reported a range of emotions when caring for people who $\mathrm{SH}$, from both frustration and empathy at attending such 'regulars', to melancholy, futility and fear when a person known to them had died by suicide, fear of litigation and blame from their employers and others, and sadness at the loss of life. 
Stigma may be defined as a process involving labelling, separation, stereotype awareness, stereotype endorsement, prejudice and discrimination in a context in which social, economic or political power is exercised to the detriment of members of a social group (Link \& Phelan 2001). There is evidence to suggest that services and treatment outcomes can be adversely affected by stigmatising attitudes and behaviours (Feldman 1988). Whilst all the paramedics in our study denied negative attitudes towards $\mathrm{SH}$, many of the interviews revealed stigmatising and value laden judgements, along with a prejudicial and discriminatory context surrounding the care for people who SH. This discriminatory context of care for people who SH is evident in challenges paramedics in our study reported in accessing appropriate care for people who SH. One may also consider the limited training and education on SH care that paramedics received in caring for people who SH to be discriminatory, when they report the over-emphasis of their training and education in relation to physical care such as trauma and life-threatening emergencies.

Parity of esteem is a principle that can be defined as: 'Valuing mental health equally with physical health' (RCPsych 2013). A longstanding lack of parity between mental and physical health problems exists in healthcare, and this has been recognised to be inequitable and socially unjust (RCPsych 2013). It is argued by the author of this thesis, that at the heart of the lack of parity of esteem in paramedic care for people who $\mathrm{SH}$ is the social value afforded to people who $\mathrm{SH}$ from paramedics, the health systems they work within and wider society. Paramedics in the present study report how $\mathrm{SH}$ receives less attention in their education system, which focusses on physical conditions such as major trauma which is less common in their practice than $\mathrm{SH}$. Paramedics also report the lack of support and referral pathways for SH. Such lack of attention to $\mathrm{SH}$ may be reinforcing stigmatising, discrimination and the presence of value laden judgements in paramedic care for people who SH.

Phrases and language used by paramedics in the interviews terms such a 'regulars', 'time wasters', 'repeat offenders' and 'committed self harm' revealed and reinforce stigmatisation of people who SH. Sommer-Rotenberg (1998) gives an example in her writings about the use of language in suicide, urging careful consideration of the words used to describe this destructive act, particularly the phrase "commit suicide" 
(p.239). Sommer-Rotenberg (1998) argues that the only acts we commit are heinous ones: adultery, a felony or crime, and that it is such connotation of illegality and dishonour which intensifies the stigma, especially in those attached to the one who has died and those who have been traumatized by this loss. Sommer-Rotenberg (1998) tells how tuberculosis was romanticised in the 19th century, becoming a metaphor for sensitivity and creativity, whereas suicide, by contrast, has been demonised as a metaphor for moral weakness or failure, and how people consider any form of psychological vulnerability as a moral lapse. Such stigmatising language towards SH within society and interviews with our paramedics may be a reflection of the low social value placed on SH. This language again has connotations for SH in terms of the role of the sick described by Talcott \& Parsons (1958 p. 176), and the deliberate and self-destructive nature of SH being viewed as an affront to the principles paramedic work to, their training and the emergency care system they work within, all of which are aimed at saving life.

Studies have reported how stigma impedes help-seeking (Schomerus \& Angermeyer, 2008; Thornicroft 2008). A systematic review conducted by Gulliver et al (2010) of barriers and facilitators to mental health help-seeking found key barriers include stigma, lack of accessibility and fear/stress about the act of help seeking or the source of help itself. All these aspects were revealed in the present study. Corrigan (2004) also suggests that stigma may deter help-seeking through two routes: (1) by people wanting to avoid the label that receiving formal care often brings, and (2) by the desire to avoid experiencing internalised stigma such as shame and embarrassment. The language used by interviewed paramedics, lack of parity of care for SH versus physical presentation, limited training and access to care pathways, and surreptitious strategies such as 'ways and means' of detaining people who $\mathrm{SH}$, are all examples of how stigmatising and prejudicial beliefs, practices and emotional reactions of staff, make it more likely that individuals who SH may be discriminated against and deter future help seeking from people who SH (Allen 1995).

Overcoming concerns of patients and paramedics, changing perceptions and reducing stigma towards people who SH so that they are more willing and able to access services, may reduce the discriminatory context which appears to currently persists 
in paramedic care for people who SH. Through their leadership, paramedics can send a powerful message to colleagues, patients and society at large to improve care for people who SH. Rejection of terms such as "commit suicide" may help to replace shame with discussion, interaction and insight, and through the use of compassionate language when they encounter or refer to people who SH paramedics may be better able to help those who $\mathrm{SH}$, which may also support the prevention of suicide.

\section{Compassion fatigue}

In a study by Gillespie and Melby (2003), 56\% of nurses working in acute medicine and $20 \%$ in ED reported emotional exhaustion. The authors conclude that regular episodes of work related stress may cause nurses to lose their ability to respond empathically to their patients. Empathy, distress tolerance and kindness are key attributes of compassion (Neff 2003), and whilst sophisticated and empathetic accounts were revealed between paramedics and people who SH (especially regulars), many reported a lack of such empathy, tolerance and kindness in their colleagues, and sometimes themselves.

The psychological sciences view compassion as recognising own or another's distress, and making an attempt to alleviate it (Gilbert, 2009). However, repeated and regular exposure to traumatic events such as $\mathrm{SH}$ places paramedics at risk of developing what has been termed compassion fatigue (CF). CF has been described as a negative effect in the professional caused by working with traumatised people (Bride 2007), resulting in an inability to provide compassionate care (Coetzee 2010). It was first applied in nursing by Joinson (1992) to describe nurses' responses of either emotional distancing to turn off their own feelings, or feeling helpless and angry as they watch patients go through trauma or devastating illness. CF can lead to numerous negative outcomes in practitioners such as burn out (Maytum, Heiman, \& Garwick 2004), apathy, a desire to quit, decreased productivity and staff turnover, and can also lead to ineffective or deficient patient care, callousness and indifference toward patients and co-workers (Coetzee \& Klopper, 2010). Exposure to continual change, cutbacks, increased workloads and pressure to meet NHS targets augment pre-existing stress and have also been attributed to CF (Illes 2011, Beaumont et al 2015). 
If left unchecked, compassion fatigue can lead to sustained changes in the ability to provide compassionate care (Coetzee 2010). Whilst the ever-increasing demands and pace of modern paramedic work is unlikely to reduce, policy makers, educationalists, legislators and leaders may accept the findings in this study and consider their ability to mitigate factors influencing CF. It is also essential for paramedics to be aware of work stressors and warning signs of compassion fatigue in themselves and colleagues so that they can be addressed through activities such as reflection, education and maintaining a healthy work-life balance to restore compassion satisfaction.

\section{Access to care}

Paramedics sought access to Crisis Resolution Teams (CRTs) for people who SH; Paramedic 6 asked:

"Shouldn't there be a facility that we can refer directly to a mental health crisis team unit” (Para 6)

CRTs were considered by paramedics a more appropriate option than the ED in many cases. Despite this, paramedics reported being unable to access or refer people to these teams. Whilst it was discussed earlier that alcohol use in SH may exclude referral to services such as CRTs, it was reported by paramedics in our study that many patients choose not to attend the ED and may refuse care, possibly due to previous negative experiences (RCPsych 2008, NCCMH 2004). Boscarato et al (2014) found that mental health consumers are still encountering inconsistent crisis responses, highlighting the need for more intensive and tailored responses to be developed. This approach reflects the range of options of support for paramedics in their care of people who SH called for in policy (RCP 2006, 2008, NICE 2004, Mental Health Crisis Care Concordat 2014). Alternatives to hospital following SH are said to deliver higher levels of user satisfaction, with no difference in short-term clinical outcomes (Slade et al 2010). Along with CRTs, initiatives such as suicide crisis phone lines are also showing promise for reducing distress and linking callers to services (Britton et a 2013, Gould et al 2012). However, many people who paramedics encounter with SH may be eligible for such services, which could form part of a tailored response from paramedics. The development of any such tailored services or alternatives to ED should, however, recognise the need for patients to 
receive a psychosocial assessment before discharge, called for by NICE (2004), and access to specialist follow-on care to enable them to deal with their SH behaviour.

\section{Training and education for Paramedics in SH care}

The NICE (2004) guidelines call for ambulance staff to be trained in the assessment and management of SH but, despite this, from the results of this study, it appears that this is not happening in practice. Five of the paramedics interviewed had received education specific to $\mathrm{SH}$, yet all the paramedics interviewed recognised there was need for further training and education in this area. This lack of training and education is consistent with previous studies reviewed in the metasynthesis and systematic review (Rees et al 2014, 2015), which report between $75 \%$ to $90 \%$ of staff had not received any training (McAllister et al 2008, Egan 2012, McLaughlin 1994).

Whilst a lack of training in care for people who SH was raised by paramedics they also emphasised how they received significant amounts of training on other less common conditions. Paramedic training was initially conceived for life threatening conditions such as cardiac emergencies and major trauma. It is estimated there are 20,000 major trauma cases each year in England (NAO 2010), whilst the number of emergency 999 calls presenting to English Ambulance services now stands at 9.00 million a year (Health \& Social Care Information Centre 2015). Major trauma is therefore only a small proportion of the ambulance service's workload (NAO 2010) at approximately $0.2 \%$ percent of calls. Conversely mental health calls constitute up to approximately 10 percent of calls to ambulance services (INVENT 2013, Munjal et al 2011, Cuddleback 2010), and of these 53\% have been found to be SH (INVENT 2013). Such a disproportionate focus on training in cardiac and trauma care at the expense of training to care for people with mental health problems may not be justifiable.

Paramedics interviewed reported that education should focus on issues such as why people SH, legislation and decision making surrounding SH. Paramedics linked their lack of education in SH to uncertainties in decision making, and the surreptitious strategies and 'ways and means' which have evolved around paramedic care for people who SH may be due to a gap in the training, competence and legislation, and 
an example of a hidden curriculum where ethical principles and virtues are taught through on-the job socialisation by peers (Lempp \& Seale 2004). Within UK paramedic practice, such education through socialisation has many risks; it may reinforce myths around $\mathrm{SH}$, stigma, judgments and discriminatory practices. These issues may be addressed through formal and sustained education.

Paramedic education and practice has evolved significantly in a short period of time, from short vocational training courses to professional registration with the Health \& Care Professions Council and higher educational development through a full BSc. The College of Paramedics Curriculum Guidance (CoP 2017) provides the most upto-date guidance on paramedic education and training, and makes a raft of recommends around mental health care including placements where mental health care is being delivered, such as crisis teams, and street triage. This is important as there is evidence that attitudes to $\mathrm{SH}$ amongst health professionals are more positive in those prepared through higher education rather than vocational training (McCarthy et al 2010, Sun et al 2007). Despite this, over half the paramedics in our study had not have received such higher education, which may be reflective of the wider paramedic workforce.

Paramedics in our study reported lack of confidence and understanding in caring for people who SH and recognised this need for education around aspects such as decision making and legislation. Indeed, education for practitioners in the care of people who $\mathrm{SH}$ has been shown to provide significant improvements in knowledge, confidence and attitudes towards SH (Crawford 1998, McCarthy et al 2010, Friedman 2006, McCann et al 2006, Turnbull \& Chalder 1997). Knowledge and confidence have also been associated also with significant improvements in perceived personal effectiveness and reduction in negativity in dealing with people who SH (Egan et al 2012). It would appear therefore that such education would be beneficial to paramedics in their care of people who $\mathrm{SH}$.

\section{Case management and joint Police mental health working initiatives}

The Grounded Theory in this thesis reflected the 'Wicked Complexity in Paramedics' care provided to people who Self-harm', and within this grounded theory the BSP of 
'Decision making in a context of risk' was forwarded. Therefore, to improve paramedic care for people who $\mathrm{SH}$, such complexity and basic social processes need to be recognised and addressed accordingly. It was previously discussed how people who SH frequently call ambulance services; nearly half of these callers present with mental health issues including SH (Edwards et al 2015). Ambulance services are also thought to serve as a safety net in society for persons with mental health problems (Knowlton et al 2013). Case management programmes for patients with complex medical and social problems have been introduced for people who frequently attend ED. These programmes involve multidisciplinary teams and individualised care plans appropriate to the patient involving the community for ongoing care and follow-up (Pope et al 2000, Sadowski et al 2009). Such case management has been associated with reductions in both ED use and overall costs of care (Pope et al 2000, LaCalle et al 2010, Shumway et al 2008). However, studies have been conducted which show no impact on ambulance costs, despite reducing ED attendances (Okin et al 2000, Lee et al 2006, Spillane et al 1997, Pines et al 2011).

Case management initiatives for frequent callers are also being introduced into ambulance services. Rinke et al (2012) conducted a pilot case management intervention for frequent emergency medical system users and found the number of transport responses and non-transport responses were significantly reduced, with a $31.6 \%$ and $79.2 \%$ reduction respectively. The London Ambulance Service (LAS) have also introduced a system for managing frequent callers (Edwards et al 2015), called the Patient-Centred Action Team (PCAT) which employs a variety of approaches to manage the behaviour of frequent callers, depending on the patient's needs and behaviour. Edwards et al (2015) conducted a retrospective review of LAS case management data from a 2-year period (from 1 April 2009 to 31 March 2011) aiming to profile frequent callers to the ambulance service and evaluate the impact of this case management interventional approach on frequent caller behaviour. $43 \%$ of frequent callers were categorised as having acute/chronic mental health issues, $16.4 \%$ of which involved SH. They also reported most frequent callers $(86 \%)$ had multiple and complex reasons for calling, including frequent medical need, acute or chronic mental health condition, older age and unmet personal or social care needs. A significant reduction in median call volume was observed from pre-intervention to 
post-intervention. Case management initiatives of frequent callers to ambulance services may therefore have a role in addressing complexity and perceived system failures in paramedic care of people who SH. However, prior to more widespread adoption, further research is required to explore the clinical, cost effectiveness and patient satisfaction of such initiatives.

Paramedics in our study reported their close working relationship with the police when caring for people who SH; whilst mental health problems constitute up 10 percent of calls to ambulance services in Wales and internationally (INVENT 2013, Munjal et al 2011, Cuddleback 2010), it has been estimated that incidents linked to mental health also account for up to $40 \%$ of police time, and such encounters rose by 33\% between 2011 and 2014 (Guardian 2016, Brown 2013). Joint mental health and police mobile response units have been implemented across the world, in Canada (Forchuk et al. 2010, Kisely et al. 2010), the US (Lamb et al. 2002, Zealberg et al. 1992) and Australia (Allen Consulting Group 2012). Intensive mental health training for frontline police has also been introduced in the US (Watson et al. 2008) and Australia (Laing et al. 2009) with some success. Protocols have also been developed in Australia to enhance the effectiveness of communication and collaboration between police and mental health services (Department of Health \& Victoria Police 2010)

Initiatives involving the police, paramedics and mental health workers in improving care for people who SH report varying degrees of success, and Lee et al (2015) recognises the limited published research on the effectiveness for their use in managing short-term crises. Hupert (2015) sought to review internationally recognised models of police interactions with people experiencing mental health crises. The aim was to develop, implement and review a partnership model trial between mental health and emergency services that offers alternative response pathways with improved outcomes in care. Three unique models of police and mental health partnership in the USA were reviewed and used to develop the PACER (Police Ambulance Crisis Emergency Response) model. PACER operated in the south-east suburbs of metropolitan Melbourne (Allen Consulting Group 2012). Threatened suicide were the largest patient group face by PACER at 33\%. PACER 
resulted in reducing the time to assessment, was less costly and resulted in fewer patients being transported to hospital ED for care (19\% of cases with PACER; $82 \%$ of cases with usual care) than standard care. They also found significant improvements in response times, interaction and outcomes for people in crisis compared with usual services. Hupert (2015) concluded that the pilot showed that a partnership involving mental health and police services in Melbourne, Australia could be replicated based on international models.

Given previous successes of joint police-mental health response units, Lee et al (2015) conducted an evaluation of a variant of PACER (termed A-PACER) in inner city Melbourne. They again reported that a joint police-mental health mobile response unit enabled rapid delivery of a multi-skilled crisis response in the community. The most common reasons for referrals to this unit were threatened suicide (33\%). The responses included direct admission to a psychiatric unit (11\%), transportation to ED (32\%), and community management for the remainder (57\%); police officers were highly supportive of the model. Scott (2000) evaluated another such mobile response programme in Georgia in the US, and found a $23 \%$ reduction in costs per case, and an increase in the proportion of cases avoiding psychiatric hospitalisation (55\% with a joint response; $28 \%$ with traditional police response). Kisely et al (2010) also demonstrated that such a response can reduce time police spent responding to an incident and increased patient engagement in treatment.

Joint police mental health initiatives may also have a role in providing support to paramedics, addressing complexity and perceived system failures in paramedic care of people who SH. However, prior to more widespread adoption further research is again required to explore clinical, cost effectiveness and patient satisfaction of such initiatives.

\section{Caring for people who frequently present with $\mathrm{SH}$}

Paramedics in the study talked of their frustrations of frequently attending the same person following SH. Frustration such as this in caring for people who SH has been reported in other studies. Anderson et al (2003) revealed the frustrations that doctors felt with regular attenders at medical wards following SH. A futility to their care was 
also noted by paramedics, where these patients often continued to $\mathrm{SH}$, despite the care they provided to them. Such futility and frustration has also been reported elsewhere as Hopkins (2002) told how nurses became cynical and sick of dealing with the same patients time and time again following SH. These attitudes were also articulated in the theme of 'frustration, futility and legitimacy of care' within the metasynthesis.

Paramedics in this study used the term 'regulars' for people who frequently call for an ambulance. Terms such as this have been reported previously in the literature as being used by paramedics. Robertson \& Henderson (2009) recognise the term "frequent flyer" as a term sometimes used in the Australian EMS industry to describe paramedics attending the same person on a regular basis for known or suspected mental illness. Paramedics in the present study did however also report empathetic accounts of patients they frequently attended for $\mathrm{SH}$, with whom they were often on first name terms. Such relationships have also been previously recognised in the literature by Winkler (2013) who reported how there are frequent flyers, or users that utilise the EMS system so much that they are known by name by many of the providers.

This present study is the first to report how, in the context of $\mathrm{SH}$, some of the assumptions, frustrations, language and judgements made with regards to such regular users of ambulance services by paramedics may be inaccurate, stigmatising and discriminatory. It is discussed later in this thesis how these issues may be negatively impacting on care provision. Inappropriate use of ambulance services has been long recognised (Murphy 1998, Malone 1995, Richards \& Ferrall 1999, Pennycook et al 1991), and stigmatising and inappropriate terms such as abuse, or misuse of ambulance services have also been used in the literature (Kirkby \& Roberts 2012).

Perceptions of paramedics in our study about the volume and frequency of SH calls appear to be accurate and reflected in the literature. Studies have indeed shown that up to one-fifth of callers to EMS were frequent users (Knowlton et al 2013), and about two-thirds of these had evidence of behavioural health problems (Broxterman 
et al 2007, Knowlton et al 2013). Edwards et al (2015) conducted a retrospective review of London Ambulance Service (LAS) data from a 2-year period (from 1 April 2009 to 31 March 2011) aiming to profile frequent callers to the ambulance service and evaluate the impact of a case management interventional approach on frequent caller behaviour. $43 \%$ of frequent callers were categorised as having acute/chronic mental health issues and $16.4 \%$ of patients had suicidal intentions/self-harm as their presenting complaint. Mental health problems also constitute approximately 10 percent of all calls to ambulance service across the world (INVENT 2013, Munjal et al 2011, Cuddleback 2010), and of these 53\% have been found to be for SH (INVENT 2013). People who SH may therefore frequently call ambulance services and also constitute a large cohort of the total population of those who frequently call ambulance services.

There are many reasons why frequent users choose to call ambulance services. Pallazzo et al (1998) found that only $60 \%$ called because they believed they had a serious or life-threatening condition. Booker et al (2014) identified reasons for calling for an ambulance which included: perceived or actual barriers to accessing urgent care services, previous negative experience of urgent care services, the perception that the ambulance service could provide rapid triage and assessment, patient and carer anxiety, and carers' feelings of responsibility and helplessness. Paramedics in the present study reported how both themselves and patients faced barriers in accessing care, yet the assertion that frequent use of services is caused by limited access to primary care, may not necessarily be correct.

High users of ED services are also high users of non-ED services (Lacalle 2010, Hansagi 2001). Researchers in Ireland reported that frequent ED users made more visits to their general practitioners in the year of study compared to control patients and a higher proportion of these individuals also used community welfare services, social workers, addiction counselling, and psychiatric services (Byrne et al 2004). Indeed, Byrne et al (2004) also found that adults who made three or more visits to their primary care doctors were five times more likely to be frequent ED users than those who made no such visits. This may also be the case with regular users of ambulance services. It may also be the case that patient-level factors, such as disease burden, are important drivers of frequent utilisation of ambulance services. Mason 
(2014) cautions about the risk that frequent callers, once identified by ambulance services, are simply passed to another service so that budgetary rather than patient needs are met. Many patients will be frequently calling ambulance services because they are not receiving adequate care, as opposed to calling for vexatious reasons (LaCalle et al 2010).

Frequent users of ambulance services may not therefore present some benign lowrisk group of patients; they are in fact a high-risk group. Fuda \& Immekus (2006) found that frequent ED users were more likely to die after their last visit (2.6\% of frequent users vs. $1.1 \%$ of infrequent users), were hospitalised at higher rates (18.8\% vs. $14.2 \%)$ and were transported to the ED more frequently by ambulance (18.6\% vs. $12.1 \%)$. Frequency of use of services in SH may also be an indication of heightened risk of suicide, as O'Neil et al (2014) found a pattern emerged with regard to help seeking in patients in the month prior to death by suicide. In the study by O'Neil et al (2014), those aged between 20 and 29 years engaged in services more frequently in the two months prior to death (8.2\%), the 60-69 years age group were most frequent service users in the period up to four months preceding suicide (7\%), while individuals aged below19 years were more likely to access services between six months and one year prior to death $(6.6 \%)$.

Fundamental problems therefore emerge in paramedic care for people who SH when equating frequent use with misuse. It was reported by paramedics interviewed in the present study how many of the patients they encountered following SH had accessed primary care prior to calling for an ambulance, but that this did not resolve their issues. It could also be the case that people who SH and call for an ambulance have complex issues which are challenging to address. Paramedics interviewed talked about the complexity of determining whether there were any underlying physical conditions or risk of suicide and their lack of competence in in areas of mental health, all of which impacted on their decision making. Paramedics were not prepared to take risks with such patients. Whilst incidents involving frequent compared to non-frequent users are significantly more likely to be transported to an ED by ambulance (Knowlton et al 2013, Fuda \& Immekus 2006), conveyance to ED for $\mathrm{SH}$ is reported to be over $95 \%$, including when the paramedic recorded that the 
patient stated there was no suicidal intent (INVENT 2013). Such high rates of conveyance to ED for regular patients, and those who SH may reflect the aims of paramedics to reduce risks to themselves and their patients. Given the lack of other referral options and heightened risks, rather than being a waste of ambulance resources, frequent callers who $\mathrm{SH}$ may present an opportunity to prevent future $\mathrm{SH}$ and suicide.

\section{Strengths and weaknesses}

The study was conducted in one ambulance service setting, with a small sample of paramedics. This small sample size and variability of cross-sectional design, may be considered limitations, as there was a potential for selection bias, non-response bias and volunteer bias which may have occurred at particular points in time and service delivery configuration. Whilst only paramedics were included on our study, other grades of ambulance staff may be involved in care with people who $\mathrm{SH}$, however paramedics' training and professional registration gives them added responsibilities in caring for people who $\mathrm{SH}$, which were of importance in this study.

Participants were drawn from a diverse demographic, and their experience ranged between one and thirty-eight years of frontline paramedic care. This range of age and experience is considered a strength in the study, as we were looking for variety of experience and whether our emerging theories held true across this variety which is one of the basic principles of GTM. Women have dominated in samples in previous studies of SH reported in the systematic review of the literature review and metasynthesis (Rees et al 2014, 2015). This may be due to many studies involving nursing, which has historically been a profession dominated by women. There is currently a ratio of approximately1:3 women to men registered paramedics in the UK (HCPC 2018), and this is reflected in the wider emergency ambulance workforce internationally (Nesta 2018). The purposeful sampling in this study of a ratio of 1:3 women to men reflects the demographic of the current UK and international paramedic workforce and is again considered to be a strength of the study.

The study relied on small numbers and was not a positivist study such as a randomised controlled trial, and therefore one should be careful making inferences. 
Positivist methodologies rely on measuring variables, using experimental designs and testing hypotheses to provide causal explanations (Sarantakos 2005). However, this study pioneers new ground by applying the interpretivist-constructivist perspective of EGTM to paramedic and pre-hospital care research. The interpretivistconstructivist perspective adopted in this study sees the world and reality as being subjective, multiple, socially constructed by its participants, interpreted and experienced by people in their interactions with each other and with wider social systems (Lincoln \& Guba 2000, Krauss 2005). Both SH and paramedic care are enacted in a social world, and within a care context which has many influences and is complex. EGTM therefore provided an opportunity to meet the aims of this study by enabling exploration of paramedic care of this patient group, their perceptions of the act of SH and allowed for the emergence of understandings and construction of meaning.

The philosophical and epistemological basis of the study, and its constructivist methodology of EGTM, influenced every aspect of the study; its conduct, subsequent findings, dissemination and impact. Opting to sacrifice the objectivity and generalisability which can be gained from positivist approaches to research resulted in construction of knowledge and theory development from one group of paramedics in one ambulance service and may be considered limited through its construction by an experienced paramedic researcher, who was a relative insider, researching his own professional group. The author of this thesis and primary researcher was a paramedic and had significant influence over the research agenda, which may be considered a potential bias, as the researcher held a privileged position. However, this was counteracted by providing a detailed description of the role of EGTM, and undertaking activities such as systematic reviews and metasynthesis of the literature, coding checks and discussions with other members of the team who contributed to interpretation of interviews.

In EGTM such insider-ness may be considered a positive, with the researcher being what Lincoln \& Guba (2005 p. 196) describe as a "passionate participant as facilitator of multi-voice reconstruction". This state of insider-ness also influenced theoretical sensitivity, which in this thesis was recognised as a multi-dimensional 
concept which included the researcher's level of insight into the research area, how attuned he was to the nuances and complexity of the participants' words and actions, the ability to reconstruct meaning from data generated with the participant, and a capacity to "separate the pertinent from that which isn't" (Strauss \& Corbin, 1990, p. 44). Theoretical sensitivity was not only restricted to the researcher's background as a paramedic, but each poster or oral presentation, position statement, publication, and their subsequent feedback and influence would have impacted on such theoretical sensitivity, and the researcher's refinement and construction of the evolving theory; this again was seen as a positive aspect of the constructivist nature of the thesis.

The systematic review of the quantitative literature and metasynthesis of qualitative literature revealed the limited published evidence in this area. They also served to further sensitise the area of study and development of the interview guide. The metasynthesis again followed the interpretive constructivist approach, because unlike synthesis of quantitative research, the metasynthesis did not involve data aggregation or secondary analysis of raw data; rather, the goal was interpretive. It must again be recognised that such an approach in this EGTM fundamentally differs from classic GTM, where Glaser (1967) claims that the researcher must be a tabula rasa, or blank slate, when entering a field of inquiry to develop theoretical sensitivity legitimately, whereas EGTM of Strauss and Corbin (1998) used in this study leaves behind this traditional, and it is recognised that the theory was interpreted by the paramedic researcher and a wider team, and the literature review and metasynthesis influenced much of its final construction. The interview guide was informed by the metasynthesis and systematic review, thus sensitising and focussing the area of study to explore aspects within a paramedic context and the areas which emerged from the literature. This was in line with Strauss (1998) who advocates the use of structured questions within interviews to lead a more forced emergence of theory. The final GT was not therefore restricted only to data collection, coding and analysis of paramedic interviews. 


\section{Insider status of the researcher}

The author of this thesis, and primary researcher, had been an operational paramedic for over twenty years before embarking on the research. He had been a prominent individual in the development of paramedic practice and education for many years, teaching in small group settings and large lectures. The paramedic community is a small professional group and due to the main researcher's length of service and support of education, he had on occasions crewed an ambulance with three of the participants, and others may have attended meetings and lectures led by him. Whilst the researcher was a paramedic at the initiating stage of the study, within the lifetime of the study he had left to work with another employer in a hospital, eventually returning as a senior leader in the Ambulance Service.

Professionals researching their own professional group such this are known as insider-researchers and are those who belong to the group they choose to study and may be embedded in a shared setting (Breen 2007, Smyth and Holian 2008). There were many benefits and drawbacks of being an insider within the context of this study. Insider researchers have a great deal of knowledge which can take an outsider a long time to acquire, they can also be aware of the politics of the institution, such as formal hierarchies, how best to approach people, or how it really works (Smyth \& Holian, 2008). Insider researchers can have a "feel for the game" or the "hidden rules" (Bourdieu 1988 p. 27). The insider-ness of the researcher was beneficial to the present study in many areas. For example, the researcher was aware of the quasimilitaristic hierarchy of the ambulance service, and for participants to freely express their views, anonymity would have to be assured. Interviewing was therefore conducted in a non-ambulance service setting to maximise recruitment by ensuring maintenance of such anonymity. Bonner and Tolhurst (2002) identified the following three advantages of being an insider-researcher: (a) having greater understanding of the culture being studied; (b) not altering the flow of social interaction unnaturally; and (c) having an established intimacy which promotes both the telling and the judging of truth. All of these advantages were beneficial in the present study; the transcripts do indeed reflect such intimacy and flow of interaction, and as the researcher in his career had experienced many encounters similar to those expressed by the paramedics interviewed, he was convinced of their validity. 
Insider researchers however can be emotionally connected to the research participants (Sikes 2008). As the researcher had cared for many people who had conducted SH or died by suicide, the researcher often reflected on these cases throughout the study and could extend an empathetic response during moments of melancholy with paramedic interviewees. The interview with Paramedic 4 was one such melancholic encounter, involving the sad case of a person whom he had attended several times for $\mathrm{SH}$, and died following one of his attendances. Paramedic 4 reported powerfully the challenges he faced in getting the right care for the patient who was also his nephew. He told of the frustrations his family had in getting his nephew to access care, and how on the night of his subsequent death he had attended him in an intoxicated state and that following discussions with a doctor and the family he was told to leave him to sleep it off. Paramedic 4 became emotional during the encounter. This case again provides a profound example of where boundaries between the researcher and the researched were blurred.

The background of the researcher being a paramedic for many years may have influenced the yielding of deep insights into paramedics' perceptions of care for people who SH in a way that an interviewer from another background may not have. The interview with Paramedic 4 was a turning point in the study, bringing with it a sense of awesome responsibility on the researcher, not only to present a truthful and rich account of such challenges for paramedics, but also to work to realise the greatest impact from the study. Paramedic 4 was offered further support in the form of staff support and counselling, along with further calls from the researcher to discuss the use of data, and any further needs he may have.

The researcher continues to encounter study participants occasionally, in many settings, as a senior leader in the ambulance service, during training events and informal social gatherings. Mercer (2007) argues that experiences such as these support the notion that researchers have multiple identities and may be simultaneously insiders and outsiders (Adler 2004, Bridges 2002). In the case of the researcher of this study, a complex mix of fluctuating insider-ness and outsider-ness continues to prevail. 


\section{Dissemination and potential impact}

Research increasingly faces challenges in demonstrating its value to society, as it has to compete with other areas in society for public money (Cohen et al 2015). This $\mathrm{PhD}$ was no exception to these principles as a small FALCK foundation research grant was successfully secured to support this study; there was also significant investment of time and mental effort from WAST, the researchers, supervisors and participants of the study. The value of research to society is often measured through research impact, which can be defined as: 'any identifiable 'benefit to, or positive influence on the economy, society, public policy or services, health, the environment, quality of life or academia' (HEFCE 2015 p. 26).

In the UK, evaluation of research impact exists in the form of the Research Excellence Framework (REF), which evaluates universities using peer review, case studies and metrics in a global context, considering whether impact on science and beyond has been reached (King's College London and Digital Science 2015). A core aspect of evaluating research impact is the peer review process, in which colleagues mutually evaluate their research output (Bornmann 2017). Bornmann (2017) contends that researchers can only make reliable and valid statements when their results are monitored by such qualified experts in the same field, ensuring that they meet certain standards.

Throughout the $\mathrm{PhD}$ there have been many peer reviewed activities involving the dissemination of information about the study, its design, purpose, progress, and the emerging results. These activities have included meetings, peer reviewed oral and poster presentations at conferences, publications, supporting position statements and influencing policy and legislation (Appendix N). Outputs from the study were not expected to lead to an immediate or fundamental paradigm shift in the field of paramedic care for people who SH, rather to build on an already established body of research and knowledge on $\mathrm{SH}$, some of which was synthesised, interpreted or reframed, and when combined with the findings from interviews in this study, extended into the new understandings and theory within the paramedic context, thus adding new perspectives on $\mathrm{SH}$ care. 
The Systematic Review and Metasynthesis were completed in 2014 and 2015 respectively, and after revision, they were successfully submitted and accepted for publication in the Journal of Psychosomatic Research (Rees et al 2014, 2015). At the time of publication this journal had an impact factor of 3.4. The metasynthesis has been cited in seven articles and read 426 times by an international audience, whilst the systematic review has been read 263 times by an international audience and cited in ten articles (Researchgate 2018). Following data analysis, the emergence of the category Professional, legal and ethical tensions, and the 'ways and means' paramedics employ in caring for people who SH emerged as a very important finding. Changes to mental health policy and legislation were being considered in the UK and so it was decided to publish this finding in a paper which, after peer review and revision, was successfully accepted for publication in the International Journal of Law and Psychiatry (Rees et al 2016). At the time of publication, the International Journal of Law and Psychiatry had an impact factor 1.5; the paper has now been read 108 times by an international audience. A final paper reporting on the study in its entirety has been in the journal Plos One; this paper is entitled: Paramedics' perceptions of the care they provide to people who self-harm: a qualitative study using Evolved Grounded Theory Methodology.

The articles published through peer review (Rees et al 2014, 2015, 2016, 2018) have received over 800 reads, and resulted in 17 citations (Researchgate 2018). These citations include areas such as caring for self-harming patients in general practice (Rowe \& Jaye 2017), qualitative research methods in emergency settings (Forero et al 2018), a prospective data linkage study exploring Ambulance attendances resulting from SH after release from prison (Borschmann et al 2017) and studies examining the relationship between emergency response services personnel and suicide in Canada (Koopmans et al 2017). The metasynthesis (Rees et al 2015) was also cited in a chapter of the International Handbook of Suicide Prevention exploring management of suicidal risk in Emergency Departments (Hatcher 2016).

Publications and citation counts above may provide an indication of how useful the research has been for scientists working in the same or similar disciplines 
(Moed 2005). However, whilst these measures may serve to demonstrate a degree of impact, the wider influence on policy, education and improved paramedic care for people who $\mathrm{SH}$ is less tangible.

Theories of public policy have shown that policy-making rarely occurs in neat sequential stages (Cairney 2016). Rather, Cairney (2012) argues that the policy making process is messy and non-linear, that the search for information is always incomplete, but rather, research and the use of information filters into policy making "in a large, messy policy process, far removed from that idea that the evidence has a direct input to a clearly definable policy process" (2014 p10). Subsequently, when considering the extent to which social science research impacts on policy, the emphasis is on counting the potential occasions of influence, rather than trying to chart the unmeasurable or even unknowable outcomes of this influence (LSE 2011).

The philosophical basis of this study has been discussed many times, where knowledge and theory presented within this thesis reflects the world and reality as being subjective, multiple, socially constructed by its participants, interpreted and experienced by people in their interactions with each other and with wider social systems (Lincoln \& Guba 2000, Krauss 2005). Within this context, the LSE (2011) approach to research impact in social science research is adopted, where research impact is defined as:

"a recorded or otherwise auditable occasion of influence from academic research on another actor or organization (LSE 2011 p11)

There have been many 'occasions of influence' throughout the study period, which include activities presented above which provided opportunities to communicate the study and influence other 'actors' with an interest in paramedic care for SH. Despite this, other than the peer review process, presenting 'auditable occasions' are more of a challenge. The study, and its outputs have however influenced several enquiries and policy documents, some of which are presented below, and partly addresses the aim of the study.

In 2016 knowledge from the study was drawn upon to inform the written evidence from the College of Paramedics submitted to the Home Affairs Committee's inquiry 
on Policing and Mental Health (CoP $2016 \mathrm{c}$ ). The $\mathrm{PhD}$ is acknowledged in this document, and it reflects many of the findings. $\mathrm{CoP}$ (2016 c) recognises that paramedics are often the first point of contact for those with mental health crisis and are well placed to contribute to care. Amongst many of the other findings of the study reported in this thesis, $\mathrm{CoP}$ (2016 c) acknowledges that police and paramedics may unknowingly resort to breaching legislation and professional codes of practice to safeguard patients in their care, reflecting the revelations in this thesis.

The present study was again drawn upon and referenced to support a response from the College of Paramedics to the Welsh Government Inquiry into alcohol and substance misuse (2016 b). This response aimed to influence policy and strategy in reducing harms from alcohol or substance misuse, but also highlighted the significant role of alcohol in paramedic care for people who SH. In 2018, the main researcher and author of this thesis was asked by WAST to contribute to The Welsh NHS Confederation and WAST response to the Health, Social Care and Sport Committee's inquiry into suicide prevention in Wales. This was followed by a request for the author to provide oral evidence to this enquiry. These submissions were informed by the findings of the study reported within this thesis. The written evidence, and video footage of the oral evidence are available on the Welsh Government website (WG 20018).

Despite the publications and activities above, it is recognised that it is implausible that a single study will make a significant change in paramedic care for people who SH. The implausibility that political decision-makers could somehow be a kind of blank slate on which researchers or scientists could directly inscribe the 'correct' course of action to be followed is also recognised by the researcher and others (Bastow et al 2014 p. 30). This study does however build on and contribute to a wider evidence base in the care of SH, and the papers published from this study (Rees et al 2014, 2015, 2016, 2018) constitute the only known literature specifically exploring paramedic care for $\mathrm{SH}$. Whilst this study may have resulted in incomplete understandings of paramedic care for $\mathrm{SH}$, it may also provide a potential framework for guiding subsequent empirical research (Bradley et al 2007). 


\section{Improvements in paramedic care for people who SH}

The EGTM approach to this study accepts that reality cannot be fully known but is interpreted and is linked to time and place. Throughout the six years which have lapsed since embarking on the study, the author has constructed a version of the reality of paramedic perceptions of care for people who $\mathrm{SH}$ and presented it through the GT. The journey to developing the GT has not been a static one, and within this thesis it has been acknowledged how a range of factors have evolved and influenced paramedic care for people who SH; the researchers, participants and final GT were not immune to the influence of such factors, rather, in EGTM, knowledge such as this is harnessed in constructivist methodology which acknowledges the importance of such a multiplicity of perspectives and 'truths' (Strauss, 1987; Strauss \& Corbin, 1990, 1994, 1998).

Education, professional development of paramedics, policy, legislative changes and availability of support for paramedics are some of the factors which have evolved since embarking on this study. The factors within the constructed GT of Wicked Complexity in Paramedics' care provided to people who Self-Harm have also continued to evolve throughout this study, and will continue to do so. For instance, many initiatives are being introduced by ambulance services to address usual factors, such as tiredness and frequent callers common to paramedic work. Ambulance services have introduced case management systems for dealing with frequent callers (Edwards et al 2015, LAS 2010, Lee 2006, Scott et al 2014, Morgan \& Thayre 2016). Despite these initiatives, there is limited rigorous evaluation of their impact on frequent caller behaviour.

Heightened factors included paramedics concerns about lack of support, which was felt to be more common in paramedic care for SH. This area again continues to evolve, through the introduction of innovations such as joint mental health and police mobile response units which have been implemented in many countries (Forchuk et al. 2010; Kisely et al. 2010, Lamb et al. 2002; Scott 2000; Zealberg et al. 1992, Allen Consulting Group 2012). These initiatives include the UK street triage schemes which involve dedicated mental health professionals collaboratively working with police officers, attending scenes and offering more tailored interventions, to ensure 
individuals receive the most appropriate care. These schemes may also go some way to addressing factors specific to SH and supporting the assessment of suicide risk. Once again however, such schemes report varying degrees of success and there is little published research reporting on their effectiveness, leading to calls for further research of these models of care (Lee et al 2015, Paton 2016, NIHR 2016).

Some of the solutions to the issues revealed in this study may come from rigorous positivist, quantitative research such as randomised controlled trials. Such positivist research was referred to earlier as normal science, tame-paradigmatic, with consistent ways of attacking problems (Rittel and Webber 1973). It was noted earlier that Rittel and Webber (1973) recognised how such tame problems can follow algorithmic processes for arriving at solutions to problems, and within the context of this study the suicide risk prediction tool may be considered in this light, which, when evaluated through such positivist means, are found to perform no better and sometimes worse than clinician or patient ratings of risk (Quinlivan et al 2017). Rather than an algorithm or tick box exercise, it is "empathic listening and talking" which RCPsych (2010 p.79) argues that "has key therapeutic benefits".

Unlike tame problems which are argued to be manageable problems, due to the lack of a proper focus, wicked problems however arrive with built-in complexities, making them doubly difficult to address. Rittel and Weber (1973) argue that there are no known algorithms for solving wicked problems, and simply identifying the problem can turn into a major task. Addressing wicked problems carries with it extensive economic, cultural and financial burdens and opportunities (Rittel and Webber 1973). Many examples were revealed of the complex nature of paramedic care for people who SH, and such wider economic, cultural and financial burdens were revealed in this study. These include the role of alcohol and violence directed towards paramedics, frustrations over frequency of calls to $\mathrm{SH}$, the deliberate nature of SH being counter to the role of the sick, and the way people should behave in promoting good health as described by Talcott Parsons (1958). Deeper cultural issues may also exist in paramedic practice, such as negative attitudes of paramedics towards people who $\mathrm{SH}$, use of pejorative terminology, stigmatising and discriminatory care lacking parity with physical conditions, all of which may be 
discouraging people who SH seeking care in a crisis. Many of the issues such as these revealed in the study defy one-off solutions.

\section{Implications for future research}

This study has revealed important understandings in the care of people who SH. The papers publish from this study serve as the only peer reviewed literature on paramedic care for people who SH, and when one considers that paramedics are often the first health professional encountered following $\mathrm{SH}$, this represents a significantly under-researched, yet important area in which to gain understandings. It is recognised that whilst researchers are yet to have explored paramedic care from their perspective, the results of this study and theoretical outputs illuminates many gaps in our understandings of paramedic perceptions of care for people who SH and provide the foundations for future research in this area.

Previous studies exploring views on care from people who SH and have focussed on professional groups such as nurses and doctors, yet none have sought to investigate the views of paramedics. Therefore, both quantitative studies such as surveys and indepth qualitative interviews with people who have received care from paramedics following SH may yield rich insights in order to improve care. Further research with paramedics is also warranted, such as a large-scale survey exploring their attitudes towards SH, using validated surveys instruments such as ADSHQ used in McAllister et al (2002). Future research with paramedics may involve the development and evaluation of a referral intervention for paramedic care of people who $\mathrm{SH}$; this could be based on the Medical Research Council guidelines for developing and evaluating such complex interventions (Medical Research Council 2008), and defines a 'complex intervention' as:

"one where there is ambiguity over the 'active ingredients' of the intervention and their optimal mix and the phased development of such interventions has been advocated" (p. MRC 2008).

Further research is also recommended on validation of training programmes for paramedic care of people who SH. These programmes should aim to build empathy, improve confidence and competence, and include areas such as understanding why people $\mathrm{SH}$, how to conduct a comprehensive assessment of physical, psychosocial 
and mental health, and also an understanding and application of legislation such as MCA (2005) and MHA (1983) to scenarios they encounter.

\section{Implications for policy and practice:}

- $\quad$ Paramedics urgently require further education in care for people who SH.

This education should aim to build empathy and understanding in paramedic care for people who SH. This education should also focus on real world application of legislation such as MCA (2005) and MHA (1983) and be applied to specific scenarios and challenges paramedics face difficulty with in practice, such as a patient with full mental capacity who is at risk, but refuses care.

- Guidelines for paramedic SH care should cease to advocate suicide risk prediction scores due to their lack of validity. Rather, they should support development of understanding, empathy, listening and involvement of people who SH in shared decision making.

- $\quad$ Support mechanisms are urgently required for paramedics caring for people who $\mathrm{SH}$, such as referral pathways and telephone advice. These may reduce the sense of isolation felt by paramedics and provide more appropriate alternatives to ED.

- $\quad$ Future development of mental health legislation around SH should consider the complexity of paramedic care for people who SH. Legislators should consider if, in the absence of medical practitioners, paramedics as registered health professionals are now better placed than police to have limited powers of detainment under the MHA (1983).

- $\quad$ Case management for frequent callers and joint police mental health initiatives may have a role in providing support to paramedics, addressing complexity and perceived system failures in paramedic care of people who $\mathrm{SH}$. However, prior to more widespread adoption, further research is required to explore clinical, cost effectiveness and patient satisfaction of such initiatives. 


\section{Chapter Nine - Conclusion}

The conclusion signals the end of the research report and closes with the key messages of this thesis. The study has achieved its aim of exploring paramedics' perceptions of caring for those who SH to inform education policy and practice. The study has also met all its objectives, as paramedics' perceptions of caring for those who SH have indeed been revealed, and a Grounded Theory of paramedics' perception of caring for people who SH was also constructed, reflecting the 'Wicked Complexity in Paramedics' care provided to people who Self-harm'. This theory explained how there are 'usual factors', 'heightened factors' and 'factors specific to SH' which influence paramedic care for people who SH, and the Basic Social Process in paramedic care for people who SH was deemed to be 'Decision making in a context of risk'.

Whilst the study reported within this thesis discovered new knowledge which adds to the pre-existing literature, many of these understandings were already known within other professional groups. This included the frustration and futility of care felt by staff who care for people who $\mathrm{SH}$, the limited education they receive on care for people who $\mathrm{SH}$, their confusion over legislation, poor attitudes, stigma and a lack of understanding of SH. Existing theories and the literature that were available in relation to paramedics' perceptions of care for people who SH were therefore utilised to contextualise the study which, when synthesised into the data from this study, added new dimensions and insights into paramedic perceptions of care for people who SH. The initial literature review, followed by more rigorous systematic reviews of the quantitative literature and metasynthesis of the qualitative literature, yielded no studies focussing on paramedic perceptions of care for people who $\mathrm{SH}$, and the one found which explored SH within an ambulance service context (Gohds 1978) was conducted over forty years ago, prior to the development of paramedics. These reviews of the literature reflected a significant gap in the literature with an absence of studies focussing on paramedic care for this group of patients. The literature reviews also helped to develop the interview guide, theoretical sensitivity in the researcher and the continuum of accumulation of knowledge and theory building within the study. 
The study found that patients who SH frequently present to paramedics who identify them as a particularly problematic patient group to deal with; this has been found previously in other groups such as nursing and medical staff. The study also revealed that many of the challenges and risks in caring for people who SH are present across the paramedics' whole case mix and are 'usual factors' in the care they provide; these include busyness, tiredness and the increasing number of frequent callers. Paramedics reported how many of their colleagues had negative attitudes towards people who $\mathrm{SH}$, and indeed many stigmatising views and pejorative terms were used in the interviews. It was suggested that these 'usual factors' of the busy environment paramedics work within, tiredness and the increasing number of frequent callers, may be contributing to the development of compassion fatigue $(\mathrm{CF})$ in paramedics, which is the negative effect on a professional caused by working with traumatised people and resulting in an inability to provide compassionate care.

Paramedics reported 'heightened factors' in caring for people who SH such as the difficulties they face accessing appropriate care pathways (including care from GPs and Crisis Teams), which might provide a safe and effective alternative to conveyance to hospital. Paramedics also reported the 'heightened factors' associated with dealing with patients who had consumed alcohol who SH, which was common, as $47.8 \%$ of SH injuries have been found to be alcohol-related ( $\mathrm{Li} 2007$ ) and up to $46.1 \%$ of SH patients have consumed alcohol within six hours of SH (Haw et al 2005). People who SH and consume alcohol were deemed to be difficult to assess by paramedics interviewed, who highlighted how alcohol could mask or mimic underlying medical problems such as diabetes. People who $\mathrm{SH}$ and consume alcohol were also challenging to provide onward care for, as crisis teams and mental health services would not accept referral of a patient who had consumed alcohol. The current situation where people who SH and have consumed alcohol are taken to ED therefore appears to be the safest and only option available to paramedics. People who SH and consume alcohol were also thought to heighten the vulnerability of health staff to acts of aggression and violence in such circumstances. More than $75 \%$ of ambulance personnel have experienced threats and/or violence when performing their duties (Suserund et al 2002), and such violence and aggression 
directed towards ambulance staff may be increasing (Maguire 2018). Paramedics' perceived vulnerability to acts of aggression and violence following SH further reflects the 'wicked complexity', which may carry significant burdens and societal influences to solve. Maguire (2018) reflects on such challenges with 'heightened factors' arguing that although interventions had been attempted to reduce violence against paramedics, it continues to be a growing problem, and effective solutions will likely be multifaceted and include training, engineering changes, community education and adjustments to agency policies. Maguire (2018) argued that a national commission was needed to address this growing problem.

'Factors specific to $S H$ ' included confusion from paramedics over assessing and managing physical versus mental health needs in $\mathrm{SH}$, indeed, they report uncertainty over whether a patient who deliberately harms themselves is suffering from a mental condition and paramedics reported difficulty in understanding why people SH. It was argued that $\mathrm{SH}$ challenged their role as care givers and preservers of life as the deliberate nature of SH was counter to this function. SH was also argued to violate the role of the sick (Talcott \& Parsons 1958), where the patient must try to get well.

Many of the 'factors specific to SH' related to misunderstandings and a miss-match in terms of the volume of SH in the paramedics' case-mix versus the limited attention it receives in terms of education, skills and care delivery. These issues may also be contributing to stigmatising and judgemental attitudes towards people who $\mathrm{SH}$, and prejudicial and discriminatory care. It was recognised that this was consistent with the longstanding lack of parity between mental and physical health problems which exists in healthcare, which has been recognised to be inequitable and socially unjust (RCPsych 2013). It was argued that the challenges paramedics faced may be addressed by adopting a parity of esteem approach, where mental health is valued equally with physical health (RCPsych 2013). Therefore, the education, skills and care delivery from paramedics for $\mathrm{SH}$ should receive equal attention as for physical presentations.

Paramedics had to make on-the-spot care decisions for people who SH which were complex and carried out in a context of risk. 'Decision making in a context of risk' 
was therefore deemed to be the BSP and spanned all of the categories revealed within the study. These risks included immediate risk of further $\mathrm{SH}$ or suicide, risks to the paramedic or others in terms of aggression, and risks to the paramedic in terms of the potential for loss of their own professional status should a patient come to harm after their care. Whilst risk prediction scales have been developed to support practitioners identify risk of suicide, they perform no better, and sometimes worse, than clinician or patient ratings of risk and lead to a waste in valuable resources (Quinlivan et al 2017). None of our paramedics in the study reported use of such risk prediction tools, but even so, they were not prepared to leave a patient at home following SH due to such risks. Risk aversion in ambulance staff who care for people who $\mathrm{SH}$ is also reflected in the literature, with the ambulance conveyance to ED for $\mathrm{SH}$ reported to be over $95 \%$, including when it was recorded that the patient stated there was no suicidal intent (INVENT 2013).

Surreptitious strategies between the police and paramedics were revealed involving deceiving patients into leaving their home for a public place where Section 136 of the MHA (1983) could be enacted, and the patient detained to a safe place such as ED. This scenario was recounted by many of our participants, it was also reported by the England and Wales Independent Police Complaints Commission (2015), and one paramedic in our study called it the "ways and means act". Such practices may be considered unethical, not in-keeping with the principles of professional practice, or an affront to the values of shared decision making and respecting patients' autonomy. Practices such as these may also be adding discriminatory care to the hidden numbers of people who SH or hold suicidal thoughts and do not contact health professionals. This study has however highlighted the complexity of this scenario and the reasons for "ways and means act" given the lack of alternatives for paramedics and patients.

Rittel and Weber (1973) argue that there are no known algorithms for solving 'wicked problems', and simply identifying the problem can turn into a major task. Paramedic care for people who SH is indeed a 'wicked problem', and the insights revealed in this study identify the enormity of the task of tackling the issues raised. Addressing wicked problems carries with it extensive economic, cultural and financial burdens and opportunities (Rittel and Webber 1973). Many of the issues 
revealed in this study likewise carry such burdens; this may include the role of alcohol in society, the reasons behind the increasing number of people Self-Harming, why ambulance services are attracting more regular callers, increases in violence and aggression towards ambulance staff, rationing and prioritising ambulance services due to the frustrations of paramedics caring for people who SH at the expense of others who may be more sick or deserving of care, the role of the individual in promoting their own health, parity of esteem for mental versus physical problems, legislation which has to balance the rights of the individual versus the states responsibility to save life, and many more. Whilst many of these issues are modifiable, it is wider society and health and social care policy underpinned by high quality research evidence, which will be instrumental in solving these issues, rather than this one study, an individual paramedic, ambulance service or health system.

Many of the issues revealed within this study, whilst complex, are currently being attempted to be addressed. For instance, paramedics reported frustrations at people who $\mathrm{SH}$ frequently call them. Regular callers such as these were deemed to be a 'usual factor' in paramedic care. It was indeed found that up to one fifth of callers of EMS were frequent users (Knowlton et al 2013), approximately half of these are categorised as having acute/chronic mental health issues and $16.4 \%$ of such regular patients had suicidal intentions/self-harm as their presenting complaint (Broxterman et al 2007, Knowlton et al 2013, Edwards et al 2015). Mental health problems also constitute approximately 10 percent of all calls to ambulance services and of these $53 \%$ have been found to be for SH (INVENT 2013, Munjal et al 2011, Cuddleback 2010). Schemes have indeed been initiated for managing frequent callers to ambulance services such as PCAT, which was introduced into the London Ambulance Service. Edwards et al (2015) reported how the majority of frequent callers $(86 \%)$ have multiple and complex reasons for calling ambulance services and in $82 \%$ of cases multiple interventional strategies were required.

Initiatives involving the police and mental health workers in mobile response units have been implemented across the world which are acceptable to patients and staff (Forchuk et al. 2010; Kisely et al. 2010, Lamb et al. 2002; Scott 2000; Zealberg et al. 1992, Allen Consulting Group 2012). Schemes such as PACER (Hupert 2015) and 
its variant A-PACER (Lee et al 2015) report promising results, with most patients being resolved with community management $(57 \%)$ while a minority of patients being admitted to a psychiatric unit, (11\%) or transportation to ED (32\%). These initiatives may reduce of isolation felt by paramedics, and provide more appropriate alternatives to $\mathrm{ED}$, thus reducing the need to employ "ways and means" in the care of people who $\mathrm{SH}$.

Case management for frequent callers and joint police mental health initiatives may have a role in providing support to paramedics, addressing complexity and a perceived sense of isolation and system failures in paramedic care of people who SH. However, prior to wider spread adoption further research is required to explore clinical, cost effectiveness and patient satisfaction of such initiatives.

Paramedics are health professionals registered with the HCPC and are increasingly seen as providers of high quality health care. Despite this, when caring for people who SH, medical personnel who have powers under MHA (1983) are rarely present or available, and often, the only personnel present with such powers when paramedics are caring for people who SH are the police. However, the police are not registered health professionals and therefore may rely on a non-clinical interpretation of whether a patient is suffering from a mental disorder. Such characterisation of an individual by subjective judgment of traits or behaviours may be influenced by stereotypically presumptions of how individuals with mental disorders present and can be vulnerable to human rights abuses (Fistein et al 2009). Wickremsinhe (2018) found non-existent or person-description definitions of mental disorder in $39 \%$ of laws analysed and argued that such laws may endorse human rights violations by permitting treatment without adequate assessment of disorder. UK mental health legislation may therefore need amending to rectify this situation. Future mental health legislation should consider the complexity of paramedic care for people who $\mathrm{SH}$. Legislators should consider if, in the absence of medical practitioners, paramedics as registered health professionals are now better placed than police to have limited powers under UK mental health legislation. Such limited powers should aim to hold and protect people who SH from themselves and others until the crisis is over and a full psychosocial assessment has been conducted. If any such powers 
were to be introduced, paramedics would need further education and skills in clinical and psychosocial assessment.

Education and training in the care of people who SH can improve knowledge and confidence and reduce negative attitudes towards SH for practitioners (Crawford 1998, McCarthy et al 2010, Friedman 2006, McCann et al 2006, Turnbul \& Chalder 1997, Egan et al 2012). Paramedics in this study reported a lack of such training and it is therefore argued that such training and education is urgently required. This education should aim to build empathy and understanding in paramedic care for people who SH. This education should also focus on real world application of legislation such as MCA (2005) and MHA (1983) and be applied to specific scenarios of challenges paramedics face difficulty with in practice, such as a patient with full mental capacity who is at risk, but refuses care.

A wide range of policy and guidelines recognise the role that ambulance staff have to play in SH care (RCP 2006, 2008, NICE 2004, Mental Health Crisis Care Concordat 2014). These guidelines make a range of recommendations, such as access to crisis resolution teams, approved doctors and social workers, training for ambulance staff in the assessment and early management of $\mathrm{SH}$ and agreed local pathways and guidelines. Despite such recommendation, reports from paramedics within this study reveal that this is not happening in practice. This is consistent with previous reports from health staff that guidelines for treating SH were lacking or underused (McCann et al 2006, McAllister 2002, McCarthy \& Gijbels 2010). This is of concern as it may represent a failure of national guidelines to influence paramedic care for people who $\mathrm{SH}$. The importance of this finding links with assertions that the awareness and availability of guidelines and policies will support positive relationships between knowledge, confidence and perceived personal effectiveness, decreasing negativity and encouraging an empathic approach (Rees et al 2014, McAllister (2002). Realworld adoption of guidelines may also result in a less stigmatised view of people who $\mathrm{SH}$ and discriminatory care.

The dissemination and impact of this study were presented, which recognised that the impact of such research may not be tangible or obvious, with an emphasis on 
counting the potential occasions of influence, rather than trying to chart the unmeasurable or even unknowable outcomes of this influence (LSE 2011). Many occasions of influence were presented which included peer reviewed publications, conference presentations, position statements and evidence to Government enquiries. These knowledge claims reflect the 'wicked' nature of paramedic care for people who $\mathrm{SH}$, which is both complex and multifactorial. The recommendations presented in the study provide a guide for action, and include a need for further education for paramedics, consideration of amendments to legislation, introduction of support such as PACER and case management of frequent callers, all of which should involve robust evaluation through research.

There are still many questions left unanswered by this study and further research is needed such as a large-scale survey exploring paramedics' attitudes towards SH, development and evaluation of a referral intervention for paramedic care of people who SH. Further research is warranted on the validation of training programmes for paramedic care of people who SH which aim to build empathy, improve confidence and competence and provide a comprehensive assessment of physical, psychosocial and mental health and application of legislation such as MCA (2005) and MHA (1983) to scenarios they encounter.

Finally, Rittel and Webber (1973) observe that the most which might be said about solutions to 'wicked problems' is that they have made things 'better', rather than being definitively the best. This study has revealed that paramedic care for people who $\mathrm{SH}$ is a complex care interaction which occurs in a social world, where the built-in complexities of SH collide with paramedics' lack of education, confidence and referral options and support when caring for people who SH. All this is being played out in the acute presentation of $\mathrm{SH}$, where significant harm or death by suicide are potential outcomes. The methodology and subsequent findings in this thesis therefore should be viewed from the perspective that it has produced only one of many possible constructions of paramedic perceptions of care for people who SH. 


\section{References}

Ackerman, A. Graham, M. Schmidt, H. (2008) Critical events in the lives of interns. J Gen Intern Med; 24:27-32.

Adelstein, A. Marsdon C. (1975) Suicides 1961-74. Pop Trends; 2:13-9.

Adityanjee, D.R. (1986) Suicide attempts and suicides in India: cross-cultural aspects. Int. J. Soc. Psychiatry. 32, 64-73.

Ahl, C. Nyström, M. (2012) To handle the unexpected - the meaning of caring in pre-hospital emergency care. Int Emerg Nurs; 20:33-41.

Allen, C. (1995) Helping with deliberate self-harm: some practical guidelines. Journal of Mental Health. 4, 243-250.

Allen Consulting Group Alpert, G. Dunham, R. (1999) The Force Factor. Washington D.C.: Department of Justice; 1999.

Al-Shaqsi, S. (2010) Models of International Emergency Medical Service (EMS) Systems. Oman Medical Journal. Volume 25, Issue 4, October 2010

Anderson, M. Standen, P. J. Noon, J.P (2005) A Social Semiotic Interpretation of Suicidal behavior in young people. Journal of Health Psychology. Vol 10 (3): 317331

Anderson, M. Standen, P. Noon, J. (2003) Nurses' and doctors' perceptions of young people who engage in suicidal behaviour: a contemporary grounded theory analysis. International Journal of Nursing Studies. 40 (6), 587-597.

Angus, T. (2003). Animals \& ethics: An overview of the philosophical debate. Peterborough. ON: Broadview Press.

Ankolekar, S. Sare, G. Geeganage, C. Fuller, M. Stokes, L. Sprigg, N. Parry, R. Siriwardena, A.N. Bath, P.M (2012) Determining the Feasibility of AmbulanceBased Randomised Controlled Trials in Patients with Ultra-Acute Stroke: Study Protocol for the "Rapid Intervention with GTN in Hypertensive Stroke Trial" 
(RIGHT, ISRCTN66434824). Stroke Res Treat, 2012, 2012, 385753, doi:

$10.1155 / 2012 / 385753$.

APPG (2014) All Party Parlimentary Group on Mental Health Inquiry into Parity of Esteem. Available from:

http://www.rethink.org/media/967083/appg_written_evidence_-

_emergency_care.pdf [Accessed on 25/06/2014]

Auble, T.E. Menegazzi, J.J. Paris, P.M. (1995) Effect of out-of-hospital defibrillation by basic life-support providers on cardiac-arrest mortality - a meta analysis. Ann EmergMed 1995; 25:642-8.

Bahari, S. F. (2010) qualitative versus quantitative research strategies: contrasting epistemological and ontological assumptions. Journal Technology, 52. May 2010: $17-28$

Barr, W. Leitner, M. Thomas, J. (2005) Psychosocial assessment of patients who attend an accident and emergency department with self-harm. Journal of Psychiatric and Mental Health Nursing. 12, 130-138

Bastow, S. Dunleavy, P. and Tinkler, J. (2014). The Impact of the Social Sciences: How academics and their research make a difference. London: Sage Publications.

Bastow, S. (2014). 'Open data sheds light on how universities are minority providers of commissioned research to government'. Blogpost on LSE Impact blog, 20th March 2014.

Beauchamp, T.L. Childress, J.F. (1994) Principles of biomedical ethics. 4th ed. Oxford: Oxford University Press,

Beaumont, E. Durkin, M. Hollins, C.J. Carson, J. (2015) Compassion for others, selfcompassion, quality of life and mental well-being measures and their association with compassion fatigue and burnout in student midwives: A quantitative survey. Midwifery. http://dx.doi.org/10.1016/j.midw.2015.11.002i

Bernstein, R. (1983). Beyond objectivism and relativism: Science, hermeneutics, and praxis. Philadelphia: University of Pennsylvania Press. 
Benger, J.R. Jones, V. (2008) Why are we here? A study of patient actions prior to emergency hospital admission. Emerg Med J; 25:424-427.

doi:10.1136/emj.2007.050856

Bergen, H. Hawton, K. Waters, K. (2010) Psychosocial assessment and repetition of self-harm: the significance of single and multiple repeat episode analyses. $J$ Affect Disord 2010;127:257-65.

Bergen, H. Hawton, K. Waters, K. (2012) Premature death after self-harm: a multicentre cohort study. Lancet 2012; published online Sept 18. http:// dx.doi.org/10.1016/S0140-6736 (12)61141-6

Bittner, E (1967) Police discretion in emergency apprehension of mentally ill persons. Social Problems 14:278-292, 1967

Blaikie, N. (1993) Approaches to social enquiry. Cambridge: Polity; 1993

Blair, G (2011). Japan's suicide rate is expected to rise after triple disasters in March. British Medical Journal. 343; 5839.

Blohm, M. (2007), "the influence of interviewers"' contact behavior on the contact and cooperation rate in face-to-face household surveys', International Journal of Public Opinion Research, 19 (1), pp.97-111.

Bolam v Friern Hospital Management Committee (1957) [1957] 1 WLR 582

Bonner, A. Tolhurst, G. (2002). Insider-outsider perspectives of participant observation. Nurse Researcher, 9(4), 7-19.

Booker, M.J. Simmonds, R.L. Purdy, S (2014) Patients who call emergency ambulances for primary care problems: a qualitative study of the decision-making process. Emerg Med J; 31:448-452.

Bornmann, L. (2017) Measuring impact in research evaluations: a thorough discussion of methods for, effects of and problems with impact measurements. Higher Education May 2017, Volume 73, Issue 5, pp 775-787 
Bornmann, L. (2011b). Scientific peer review. Annual Review of Information Science and Technology, 45, 199-245.

Borschmann, R. Young, J. Moran, P.A. Kinner, S.A (2017) The Health After Release from Prison (HARP) cohort study. Social Psychiatry 52(9923) April 2017 DOI $10.1007 / \mathrm{s} 00127-017-1383-\mathrm{z}$

Borum, R. (2000) Comparing outcomes of major models of police responses to mental health emergencies. Psychiatric services. May 2000 Vol. 51 No. 5

Borum, R. Deane, M,W. Steadman, H. (1998) Police perspectives on responding to mentally ill people in crisis. Behavioral Sciences and the Law.16: 393-405

Boscarato, K. Lee, S. J. Kroschel, J. (2014). Consumer experience of formal crisis response services and preferred methods of crisis intervention. International Journal of Mental Health Nursing, 23, 287-295.

Boudreaux, E.D. Cagande, C. Kilgannon, J.H. Clark, S. Camargo, C.A (2006) Bipolar disorder screening among adult patients in an urban emergency department setting. Prim Care Companion J Clin Psychiatry. 2006; 8:348-51.

Bourdieu, P. (1988) Homo academicus. Cambridge: Polity Press.

Boyle M, Koritsas S, Coles J, Stanley J. A pilot study of workplace violence towards paramedics. Emerg Med J. 2007;24(11):760-.

Bradley, E.H. Curry, L.A. Devers, K.J. (2007) Qualitative Data Analysis for Health Services Research: Developing Taxonomy, Categorys, and Theory. Health Serv Res. 2007 Aug; 42(4): 1758-1772.

Branch, W.J. Pels, R. Lawrence, R (1993) Becoming a doctor: critical incident reports from third year medical students. N Engl J Med; 329:1130-2.

Breen, L. J. (2007). The researcher 'in the middle': Negotiating the insider/outsider dichotomy. The Australian Community Psychologist, 19(1), 163-174.

Bride, B.E. Radey, M. Figley, C.R. (2007) Measuring compassion fatigue. Clin Social Work Journal. 35:155-163. 
Britton, P.C. Bossarte, R.M. Thompson, C. Kemp, J. Conner, K.R. (2013) Influences on call outcomes among veteran callers to the National Veterans Crisis Line. Suicide Life Threat Behav 2013; 43(5):494-502.

Broadbent, M. Jarman, H. Berk, M. (2002) Improving competency in emergency mental health triage. Accident and Emergency Nursing. 10 (3), 155-162.

Broadbent, M., Moxham, L., Dwyer, T., 2007. The development and use of mental health triage scales in Australia. International Journal of Mental Health Nursing. $16(4), 413-421$.

Broadbent, M. Moxham, L. Dwyer, T. (2010) Issues associated with the triage of clients with a mental illness in Australian emergency departments. Australasian Emergency Nursing Journal (2010) 13, 117-123

Brokaw, J. Olson, L. Fullerton, L. (1998) Repeated ambulance use by patients with acute alcohol intoxication, seizure disorder, and respiratory illness. Am J Emerg Med $1998 ; 16: 141-4$.

Brokaw, J. Olson, L. Fullerton, L. Tandberg, D. Sklar, D. (1998) Repeated ambulance use by patients with acute alcohol intoxication, seizure disorder, and respiratory illness. American Journal of Emergency Medicine, 16 (2), 141-4.

Brown, M (2013) Mental Health Cop evidence to the Commission on Mental Health and Policing: March 2013. Available from:

http://mentalhealthcop.wordpress.com/2013/02/13/twenty-percent/ 20 MPS

Corporate. [Accessed on 20/05/206]

Brown (2017) A venn diagram of policing, mental health and criminal justice: The 'New' Section 136. Available from: https://mentalhealthcop.wordpress.com/2017/03/03/the-new-section-136/ [accessed on $22 / 02 / 2018]$

Brown, M. (2014) Should we change the Mental Health Act 1983 for emergency services? Journal of Paramedic Practice. May 08, 2014. https://doi.org/10.12968/jpar.2014.6.5.226 
Broxterman, K. Sapien, R. Fullerton, L. (2000) Repeat ambulance use by paediatric patients. Acad Emerg Med 2000;7:36-41.

BSA (2004) The British Sociological Association Statement of Ethical Practice (BSA 2004). Available from: http://www.britsoc.co.uk/about/equality/statement-of-ethicalpractice.aspx [Accessed on 12/09/2015]

Burke, L. E., Styn, M. A. Sereika, S. M. Conroy, M. B. Ye, L. Glanz, K. Ewing, L. J. (2012). Using mHealth technology to enhance self-monitoring for weight loss: A randomized trial. American Journal of Preventive Medicine, 43(1), 20-26. http://dx.doi.org/ 10.1016/j.amepre.2012.03.016

Burke, M, Duffy, D. Trainor, G. (2008) Self-Injury-A Recovery and Evidence Based Toolkit. Bolton, Salford and Trafford Mental Health NHS Trust. Available from: http://www.google.co.uk/webhp?sourceid=navclient\&ie $=U T F 8 \#$ sclient $=$ psy\&hl=en $\&$ site $=$ webhp $\&$ source $=h p \& q=B$ urke $\% 2 C+M .+$ Duffy $\% 2 C+D .+$ Trainor $\% 2 C+G .+(2$ 008) + Selfinjury $+\% 2 \% 80 \% 93+A+$ recovery + and + evidence + based $\& r l z=$ 1R2ADRA_enGB422\&aq $=f \& a q i=\& a q=\& o q=\& p b x=1 \& b a v=o n .2, o r . r \_g c . r \_p w$. $\& f p=30418681 e 9 e 178 f b \& b i w=1345 \& b i h=529 ; 2008$. [Accessed on 02/06/2014].

Burns, J. K., Jhazbhay, K., \& Emsley, R. A. (2011). Causal attributions, pathway to care and clinical features of first-episode psychosis: A South African perspective. International Journal of Social Psychiatry, 57(5), 538-545.

Burns, N. (1989) Standards for Qualitative Research Nurs Sci Q. 2: 44 DOI: $10.1177 / 089431848900200112$

Butler, J (2012) Self-harm. psychiatric aspects of general medicine medicine 40:12 Byrne, M. Murphy, A.W. Plunkett, P.K (2003) Frequent attenders to an emergency department: a study of primary health care use, medical profile, and psychosocial characteristics. Ann Emerg Med. 2003; 41:309-18.

Caine, E.D. Knox, K.L. Conwell, Y. (2011) Public health and population approaches for suicide prevention. In: Cohen NL, Galea S, eds. Population mental health: evidence, policy, and public health practice. London: Routledge, 2011: 30338. 
Cairney, P (2016) The politics of evidence-based policy making. Springer

Caldicott, C. Faber-Langendoen, K. (2005) Deception, discrimination, and fear of reprisal: lessons in ethics from third-year medical students. Acad Med 2005; 80:86673.

Caldwell, T. Jorm, A. Dear, K. (2004) Suicide and mental health in rural, remote, and metropolitan areas in Australia. Medical Journal of Australia. 181, S10-S14.

Callaghan, S. Ryan, C. Kerridge, I. (2013) Risk of suicide is insufficient warrant for coercive treatment for mental illness. International Journal of Law and Psychiatry 36 (2013) 374-385

Callaham, M. (1997) Quantifying the scanty science of prehospital emergency care. Ann Emerg Med. 30:785-790.

Campbell R, Pound P, Morgan M, Daker-White G, Britten N, Pill R, Yardley L, Pope C and Donovan J (2011) Evaluating meta-ethnography: Systematic analysis and synthesis of qualitative research. Health Technology Assessment 15(43).

Campeau, A.G. (2009) The space-control theory of paramedic scene-management. Symb Interact. 31:285-302.

Canada, K. E. Angell, B. Watson, A. C. (2010). Crisis Intervention Teams in Chicago: Successes on the Ground. Journal of Police Crisis Negotiations. 10(1/2), 86-100. doi: 10.1080/15332581003792070

Caplan, G. (1964). An Approach to Community Mental Health. London, England: Tavistock Publications.

Carne, C.J (1999) Prehospital care - a UK perspective. British Medical Bulletin 1999, 55 (No 4) 757-766

Chan, M.K.Y. Bhatti H, Meader N, Stockton S, Evans J, O’Connor RC, Kapur N, Kendall T. (2016) Predicting suicide following self-harm: systematic review of risk factors and risk scales. British Journal of Psychiatry Oct 2016, 209 (4) 277283; DOI: 10.1192/bjp.bp.115.17005 
Chapman, A. L. Gratz, K. L. Brown, M. Z. (2006) Solving the puzzle of deliberate self harm: the experiential avoidance model. Behaviour Research and Therapy, 44, 371-394.

Charman, S. (2014) Blue light communities: cultural interoperability and shared learning between ambulance staff and police officers in emergency response. Policing and society. Vol. 24, No. 1, 102_119, http://dx.doi.org/10.1080/10439463.2013.784306

Charmaz, K. (2000). Grounded theory: Objectivist and constructivist methods. In N. K. Denzin and Y. S. Lincoln, (Eds.), Handbook of Qualitative Research (2nd ed., pp. 509-536). 1Thousand Oaks, CA: Sage

Charmaz, K. (2006). Constructing grounded theory. A practical guide through qualitative analysis. London: Sage.

Christensen, E.F. Bendtsen, M.D. Larsen, T.M. Jensen, F.B. Lindskou, T.A. Holdgaard, H.O. Hansen, P.A. Johnsen, S.P. Christiansen, C.F. (2017) Trends in diagnostic patterns and mortality in emergency ambulance service patients in 2007-2014: a population-based cohort study from the North Denmark Region. BMJ Open 2017;7:e014508. doi:10.1136/bmjopen-2016-014508

Claassen, C.A. Larkin, G.L. (2005) Occult suicidality in an emergency department population. Br J Psychiatry. 186:352-3.

Clark, E. McCann, T. (2005) Researching students: an ethical dilemma. Nurse Researcher 12 (3), 42-51.

Clarke, D. Dusome, D. Hughes, L. (2007) Emergency department from the mental health client's perspective. Int J Ment Health Nurs. 2007;16:126-131.

Cleaver, K. (2013) Attitudes of emergency care staff towards young people who selfharm: A scoping review. Int. Emerg. Nurs. (2013), http://dx.doi.org/10.1016/j.ienj.2013.04.001 
Clompus, S.R. Albarran, J.W. (2016) Exploring the nature of resilience in paramedic practice: A psycho-social study. International Emergency Nursing. doi: 10.1016/j.ienj.2015.11.006

Clouser, K. D. Gert, B. (1990). A critique of principlism. Journal of Medicine and Philosophy, 15(2), 219-236.

Coetzee, S. K. Klopper, H. C. (2010). Compassion fatigue within nursing practice: A concept analysis. Nursing Health Science. 12, 235-243.

Cohn, F. Shapiro, J. Lie, D (2009) Interpreting values conflicts experienced by obstetrics-gynecology clerkship students using reflective writing. Acad Med; 84:58796.

Cohen, G. Schroeder, J. Newson, R. King, L. Rychetnik, L. Milat, A. J. Chapman, S. (2015). Does health intervention research have real world policy and practice impacts: 21 testing a new impact assessment tool. Health Research Policy and Systems. 13, 12. doi: 10.1186/1478-4505-13-3

Cole-King, A. Green, G. Gask, L. Hines, K. Platt, S (2013) Suicide mitigation: a compassionate approach to suicide prevention. Adv Psychiatr Treat19 (4)

Compton. M.T. Berivan N. Neubert, D. Broussard, B. McGriff, J.A. Morgan, R. Oliva, J.R. (2009) Use of Force Preferences and Perceived Effectiveness of Actions Among Crisis Intervention Team (CIT) Police Officers and Non-CIT Officers in an Escalating Psychiatric Crisis Involving a Subject with Schizophrenia. Schizophrenia Bulletin. doi:10.1093/schbul/sbp146

Confidential Inquiry into Suicide and homicide by People with Mental Illness (2015) Available from: http://documents.manchester.ac.uk/display.aspx?DocID=37591. [accessed on 28/10/2018]

Conlon, M. O'Tuathail, C. (2012) Measuring emergency department nurses' attitudes towards deliberate self-harm using the Self-Harm Antipathy Scale. Int Emerg Nurs;20: 3-13. 
Convention of the Rights of Persons with Disabilities (CRPD) Available from: https://www.un.org/development/desa/disabilities/convention-on-the-rights-ofpersons-with-disabilities.html. [accessed on 13/01/2016]

Cook, S. Clancy, C. Sanderson, S. (2004) Self-harm and suicide: care, interventions and policy. Nurs Stand ;18:43-55.

Cooper, J. Kapur, N. Webb, R. Lawlor, M. Guthrie, E. Mackway-Jones, K. Appleby, L. (2005) Suicide After Deliberate Self-Harm: A 4-Year Cohort Study. Am J Psychiatry. 162:297-303

CoP (2016) College of Paramedics 2016 Annual Conference: Oral presentation. Available from: https://www.collegeofparamedics.co.uk/events/national-conference2016 Video available on line at: https://vimeo.com/162336383 [Accessed on 25/12/2016]Agenda available from:

https://www.collegeofparamedics.co.uk/events/national-conference-2016

CoP (2016 b.)Welsh Government Inquiry into alcohol and substance misuse. College of Paramedics written response Available from:

http://www.senedd.assembly.wales/documents/s36259/ASM\%2003\%20Wales\%20C ollege\%20of\%20Paramedics.pdf [Accessed on 7/10/2016]

CoP (2016 c) Written evidence is submitted on behalf of the College of Paramedics for the Home Affairs Committee's inquiry on Policing and mental health. Available from:

http://data.parliament.uk/writtenevidence/committeeevidence.svc/evidencedocument/ home-affairs-committee/policing-and-mental-health/written/11946.pdf [accessed on $22 / 08 / 2017]$

CoP (2017) Paramedic Curriculum Guidance 4th Edition - 2017. Available from: https://www.collegeofparamedics.co.uk/publications/professional-standards. [Accessed on 30/09/2018]

Corbett, S.W. Grange, J.T. Thomas, T.L. (1998) Exposure of prehospital care providers to violence. Prehosp Emerg Care. 1998;2(2):127-31 
Corbin, J. (1991) Anslem Strauss: An intellectual biography. In Social Organisation and Social Process: Essays in Honor of Anselm Strauss(Ed, Maines, D.) Aldine De Gruyter, New York, pp. 17-42.

Cordner, G. W. (2000). A community policing approach to persons with mental illness. Journal of the American Academy of Psychiatry and the Law, 28, 326-331.

Corrigan PW (2004). How stigma interferes with mental health care. American Psychologist 59, 614-625.

Coulter, A. Edwards, A. Entwistle, V. Kramer, G. A. Thomson, R. Walker, R. (2017) Shared decision making in the UK: Moving towards wider uptake. Zeitschrift für Evidenz, Fortbildung und Qualität im Gesundheitswesen. June 2017. Volumes 123124, Pages 99-103.

Coulter A, Hopkins A, Moulton B. (2017b.) Montgomery v Lanarkshire Health Board: Transforming Informed Consent. Royal College of Surgeons Bulletin [Internet]. (2017b.); 99(1): [36-8 pp.]. Available from: http://publishing.rcseng.ac.uk/doi/full/10.1308/rcsbull.2017.36. [accessed on 22/09/2018]

Cox, S. Townsend, A. Preto, N. (2009) Ethical challenges and evolving practices in research on health research. Health Law Rev.17:33-39

Crawford MJ, Turnbull G, Wessely S. Deliberate self harm assessment by accident and emergency staff — an intervention study. J Accid Emerg Med 1998;15:189722.

Creaton, A. Liew, D. Knott, J. Wright, M. (2008) Inter-rator reliability of the Australasian triage scale for mental health patients. Emergency Medicine Australasia. 20, 468-474.

Crooks, D.L. (2001) the importance of symbolic interaction in grounded theory research on women's health. Health Care for Women International. Vol.22, pp11-27

Croskerry, P (2009) A universal model of diagnostic reasoning. Acad Med 84(8): 1022-8. doi 10.1097/ACM.0b013e3181ace703 
Crosskerry P. (2013) From mindless to mindful practice-cognitive bias and clinical decision-making. N Engl J Med. 2013;368(26):2445-2448.

Crosskerry, P. (2000) The cognitive imperative: thinking about how we think. Acad Emerg Med. 7:1223-1231.

Cruz R. S. Kyte, D.G. Aiyegbusi, O.L. Keeley, T.J. Calvert, M.J (2017) Assessing the impact of healthcare research: A systematic review of methodological frameworks. PLoS Med 14(8): e1002370. https://doi.org/10.1371/journal. pmed.1002370

Csipke, E. Horne, O. (2008) Understanding self-harm. SANE. Available from: http://www.sane.org.uk/Research/SelfHarmIntro. [Accessed on 12/05/2013]

Cuddleback, G. Patterson, P.D. Moore, C.G. Brice, J.H. (2010) Utilization of emergency medical transports and hospital admissions among persons with behavioral health conditions. Psychiatr Serv. 2010; 61(4):412-5.

Cumming, E. Cumming, I. Edell, L (1965) Policeman as philosopher, guide, and friend. Social Problems 12:276-286, 1965

Curtin, M. Fossey, E. (2007). Appraising the trustworthiness of qualitative studies: Guidelines for occupational therapists. Australian Occupational Therapy Journal. 54, 88-94.

Cutcliff, J.R. Stevenson, C (2008) Feeling our way in the dark: The psychiatric nursing care of suicidal people-A literature review. International Journal of Nursing Studies 45 (2008) 942-953

Data Protection Act (1998) Available from: http://www.legislation.gov.uk/ukpga/1998/29/contents. [Accessed on 12/07/2014] de Kloet, L. Starling, J. Hainsworth, C. Berntsen, E. Chapman, L. Hancock, K. (2011) Risk factors for self-harm in children and adolescents admitted to a mental health inpatient unit. Aust N Z J Psychiatry. 45(9):749-755 
De Leo, D. Heller, T.S. (2004) Who are the kids who self-harm? An Australian selfreport school survey. Med J Aust. 181(3):140-4.

Department of Health \& Victoria Police (2010). Department of Health and Victoria Police: Protocol for Mental Health. Melbourne: Mental Health and Drugs Division, Victorian Government, Department of Health. Department of Justice: Bulletin From the Field, Practitioner Perspectives, July, 1-4 in

Dickson-Swift, V. James, E.L. Kippen, S. Liamputtong, P. (2006) Blurring boundaries in qualitative health research on sensitive topics. Qualitative Health Research. 16, 853-871.

Dinan, E. (2015) Cop uses Taser on elderly man suffering diabetic episode: 'Our police officers are not paramedics'. Available from:

http://www.rawstory.com/2015/02/cop-uses-taser-on-elderly-man-suffering-diabeticepisode-our-police-officers-are-not-paramedics/. [Accessed on 12/03/2016]

Director of Public Health (2001) Annual Report: Compendium of Health Statistics. 2001 Dyfed Powys. Available from:

http://ww.dyfpwsha.wales.nhs.uk/dirphanrep2001/conents.htm [accessed on 21st October 2014]

Dixon-Woods, M. Agarwal, S. Young, B. Jones, D. Sutton, A. (2004) Integrative approaches to qualitative and quantitative evidence. London: 2004.

Dobson, F. (1998) NHS 450 psychiatrists short of a full couch. Available from: http://www.independent.co.uk/news/nhs-450-psychiatrists-short-of-a-full-couch1160047.html [Accessed on 20/11/2016]

Dodier, N. Camus, A. (1997) L'admission des malades: Histoire et pragmatique de l'accueil a' l'ho’pital. Annales: Histoire, Science Sociales. 4; 733-763.

DOH (1999) Department of Health. HSC 1999/091: health service circular Modernisation of Ambulance Services (NHS Executive 1999) Wetherby: Department of Health, 1999. 
DOH (2001) Department of Health National Service Framework for Older People.

Available from:

https://www.gov.uk/government/uploads/system/uploads/attachment_data/file/19803

3/National_Service_Framework_for_Older_People.pdf. [Accessed on 27/12/2014]

DOH (2005) The National Service Framework For Long Term Conditions. Available from:

http://www.dh.gov.uk/en/Policyandguidance/Healthandsocialcaretopics/Longtermco nditions/Long-termNeurologicalConditionsNSF/DH_4128647 [Accessed on $27 / 12 / 2015]$

DOH (2009) Tackling demand together: A toolkit for improving urgent and emergency care pathways by understanding increases in 999 demand. Available from:

http://webarchive.nationalarchives.gov.uk/20130107105354/http:/www.dh.gov.uk/pr od_consum_dh/groups/dh_digitalassets/documents/digitalasset/dh_106924.pdf [accessed on 06/08/2015]

Domino, G. Moore, D. Westlake, C. Gibson, C (1982) Attitudes towards suicide a factor analytic approach. Joumal of Clinical Psychology 3S, 257-262

Doshi, A. Boudreaux, E. Wang, N. (2005) National study of US emergency department visits for attempted suicide and self inflicted injury, 1997-2001. Ann Emerg Med. 2005;46:369-375.

Downe, S. (2008) Metasynthesis: a guide to knitting smoke. Evidence Based Midwifery 6(1): 4-8

Doyle, L., Treacy, M.P., \& Sheridan, A. (2015). Self-harm in young people: Prevalence, associated factors, and help-seeking in school-going adolescents. International Journal Mental Health Nurs; 24(6):485-94.

Dudley, M. Kelk, N. Florio, T. Waters, B. Howerd, J. Taylor, D. (1998). Coroners' records of rural and non-rural cases of youth suicide in New South Wales. Australian and New Zealand Journal of Psychiatry. 32, 242e251. 
Duhscher, J. Morgan, D. (2004) Grounded theory: reflections on the emergence vs. forcing debate. Journal of Advanced Nursing 48(6), 605-612.

Duncan, E. Best, C. Dougall, N. Fitzpatrick, D. Evans, J. Corfield, A. Goldie,I. Maxwell, M. Snooks, H. Stark, C. White, C. Wojcik. W. (2017) Investigating the population characteristics, processes and outcomes of pre-hospital psychiatric and self-harm emergencies in Scotland: a national record linkage study.

10.1136/bmjopen-2017-emsabstracts.29

Durbin, J. Lin, E. Zaslavska, N. (2010). Police-Citizen Encounters That Involve Mental Health Concerns: Results of an Ontario Police Services Survey. Canadian Journal of Community Mental Health, 29(Supp. 5), 53-71

Dyer, O (2015) Tasers. BMJ. 351:h6070 doi: 10.1136/bmj.h6070

ECHR (1950) European Convention on Human Rights. Available from: http://www.echr.coe.int/Documents/Convention_ENG.pdf. [Accessed on $12 / 01 / 2016]$

Edwards, M.J. Bassett, G. Sinden, L. (2015) Frequent callers to the ambulance service: patient profiling and impact of case management on patient utilisation of the ambulance service. Emerg Med J. 32:393-7.

Egan, R. Kiran, M. Sarma, B.A. Meena, O'Neill, B.A. (2012) Factors influencing perceived effectiveness in dealing with self-harming patients in a sample of emergency department staff. J Emerg Med; 43:1084-90.

Elpern, E.H. Covert, B. Kleinpell, R (2005) Moral distress of staff nurses in a medical intensive care unit. Am Crit Care; 14: 523-30.

ESRC (2010 Framework for Research Ethics (FRE) Updated September 2012. Available from: http://www.esrc.ac.uk/_images/framework-for-research-ethics-0912_tcm8-4586.pdf. [Accessed on 13/08/2016]

European Court of Human Rights in Keenan v UK (2001) Keenan v UK (2001) 33 EHRR 913 
Evans, E. Hawton, K. Rodham, K. (2005) In what ways are adolescents who engage in self-harm or experience thoughts of self-harm different in terms of help-seeking, communication and coping strategies? J Adolesc; 28:573-87.

Evren, C. Dalbudak, E. Evren, B. Cetin, R. Durkaya, M. (2010). Self-mutilative behaviours in male alcohol-dependent inpatients and relationship with posttraumatic stress disorder. Psychiatry Research.186(1), 91-96.

Fagan, T. J. Cox, J. Helfand, S. J. Aufderheide, D. (2010). Self-injurious behavior in correctional settings. Journal of Correctional Health Care, 16(1), 48-66.

Faruqui, R. Afghan, S (2011) P03-445 - Suicide risk awareness in Pakistan:

Influence of religious, cultural, legal, socio-economic and interpersonal factors.

European Psychiatry, Volume 26, Supplement 1, 2011, Page 1615

Feldman, M.D. (1988) The challenge of self-mutilation: a review. Comprehensive Psychiatry 29, 429-433.

Finfgeld, D. L. (1999). Courage as a process of pushing beyond the struggle. Qualitative Health Research, 9, 803-814.

Finfgeld, D. L. (2003). Metasynthesis: The state of the art-So far. Qualitative Health Research, 13, 893-904.

Finfgeld-Connett, D. (2010). Generalizability and transferability of meta-synthesis research findings. Journal of Advanced Nursing, 66, 246-254.

Finfgeld-Connett, D. Bloom, T.L. Johnson, E.D. (2012) Perceived Competency and Resolution of Homelessness among Women with Substance Abuse Problems. Qual Health Res 2012 22: 416 originally published online 2 September 2011 DOI: $10.1177 / 1049732311421493$

Fistein, E.C. Holland, A.J. Clare, I.C.H. Gunn, M.J. (2009) A comparison of mental health legislation from diverse Commonweah jurisdictions. International Journal of Law and Psychiatry 32 (2009) 147-155 
Fliege, H. Lee, J.R. Grimm, A Klapp, B.F. (2009) Risk factors and correlates of deliberate self-harm behavior: a systematic review. J Psychosomatic Res. 66(6):477493.

FoIA (2000) Freedom of Information Act (2000). Available from:

http://www.legislation.gov.uk/ukpga/2000/36/contents [accessed on 22/03/2015]

Folse, V.N. Hahn, R.L. (2009) Suicide risk screening in an emergency department: engaging staff nurses in continued testing of a brief instrument. Clin Nurs Res. $18: 253-71$.

Forchuk, C. Jensen, E. Martin, M. (2010). Psychiatric crisis services in three communities. Canadian Journal of Community Mental Health, 29, 73-86

Forero, R. Nahidi, S. De Costa, J. Mohsin, M. Aboagye-Sarfo, P. (2018)Application of four-dimension criteria to assess rigour of qualitative research in emergency medicine. BMC Health Service Research. Dec 2018 DOI 10.1186/s12913-018-29152

Friedman, T., Newton, C., Coggan, C., Hooley, S., Patel, R., OPickard, M., Mitchell, A.J., 2006. Predictors of A\&E staff attitudes to self-harm patients who use self laceration: influence of previous training and experience. Journal of Psychosomatic Research. 60, 273-277.

Fuda, K.K. Immekus, R. (2006) Frequent users of Massachusetts emergency departments: a statewide analysis. Ann Emerg Med. 2006; 48:9-16.

Fuller-Thomson, E. Sawyer, J.L. (2009) Lifetime prevalence of suicidal ideation in a representative sample of Canadians with type 1 diabetes. Diabetes Res Clin Pract; 83:9-11.

GAfREC (2011) Guidance for researchers, sponsors, $R \& D$ offices and RECs. Available from: http://www.accord.ed.ac.uk/standardopprocs/documents/GAfRECchangestoRECs_A ugust2011_000.pdf [Accessed on: 10/10/13] 
Gall, M. D. Gall, J. P. \& Borg, W. R. (2003). Educational research: An introduction (7th ed.). Boston: Allyn \& Bacon.

Gallagher, E.M. (1998) Advance directives for psychiatric care: a theoretical and practical overview for legal professionals. Psychol. Public Policy Law. 4 (3)

Gandhi, A. (2015) Non-suicidal self-injury and other self-directed violent behaviors in India: A review of definitions and research. Asian J. Psychiatry. http://dx.doi.org/10.1016/j.ajp.2015.09.015

Gartner, A. Farewell, D. Dunstan, F. \& Gordon, E. (2008) Differences in mortality between rural and urban areas in England and Wales, 2002-2004. Health Statistics Quarterly, 39, 6-13.

Gerdtz, M.F. Chu, M. Collins, M (2009) Factors influencing consistency of triage using the Australasian Triage Scale: implications for guideline development. Emerg. Med. (Fremantle) 21: 277- 285.

Ghodse, A. (1978). The attitudes of casualty staff and ambulance personnel towards patients who take drug overdoses. Social Science \& Medicine, 12, 341-346.

Gilbert, P. (2009) The Compassionate Mind. Constable, London.

Gillespie, M. Melby,V. (2003) Burnout among nursing staff in accident and emergency and acute medicine: a comparative study. J.Clin.Nurs.12,842-851.

Glaser, B. \& Strauss, A. (1967). The discovery of grounded theory. Chicago: Aldine.

Glaser, B.G. (1978) Theoretical Sensitivity. San Francisco: The Sociology Press

Gould, M.S. Munfakh, J.L. Kleinman, M. Lake, A.M. (2012) National suicide prevention lifeline: enhancing mental health care for suicidal individuals and other people in crisis. Suicide Life Threat Behav; 42(1):22-35.

Grasner, J.T. Herlitz, J. Koster, R.W. Rosell-Ortiz, F. Stamatakis, L. Bossaert, L. (2011) Quality management in resuscitation--towards a European cardiac arrest registry (EuReCa). Resuscitation 2011; 82:989-94. 
Grover, S. Sarkar, S. Bhalla, A. Chakrabarti, S. Avasthi, A. (2016) Demographic, clinical and psychological characteristics of patients with self-harm behaviours attending an emergency department of a tertiary care hospital. Asian Journal of Psychiatry. 20 (2016) 3-10

Guardian (2016) The Counted: people killed by the police in the US. Available from: http://www.theguardian.com/us-news/ng-interactive/2015/jun/01/the-counted-policekillings-us-database\# [Accessed on 22/12/2016]

Guba, E.G. Lincoln, Y.S. (1989). Fourth generation evaluation. California: Sage Publications.

Guest, G. Bunce, A. Johnson, L. (2006). How Many Interviews Are Enough? An experiment with data saturation and variability. Field Methods, 18(1), 59-82.

Gulliver A, Griffiths KM, Christensen H (2010). Perceived barriers and facilitators to mental health help-seeking in young people: a systematic review. BMC Psychiatry $10,113$.

Hafferty F. (2008) Beyond curriculum reform: confronting medicine's hidden curriculum. Acad Med. 73:403-7.

Hammersley M. (1992) What's wrong with ethnography? London: Routledge.

Harjutsalo, V. Forsblom, C. Groop, P.H. (2011) Time trends inmortality in patients with type 1 diabetes: nationwide population based cohort study. $\mathrm{Br}$ Med J Sep $2011 ; 8: 343$.

Harriss, L. Hawton, K (2011) Deliberate self-harm in rural and urban regions: A comparative study of prevalence and patient characteristics. Social Science \& Medicine. Vol 73, Issue 2, Pages 274-281,

Hatcher, S. (2016) Management of Suicidal Risk in Emergency Departments. In O'Connor, R. C. Pirkis, J. (2016) The International Handbook of Suicide Prevention. Wiley-Blackwell. DOI 10.1002/9781118903223.ch2 
Haw, C. Hawton, K. (2008) Life problems and deliberate self-harm: associations with gender, age, suicidal intent, psychiatric and personality disorder. Journal of Affective Disorders; 109:139-148.

Haw, C. Hawton, K. Casey, D. Bale, E. Shepherd, A. (2005) Alcohol dependence, excessive drinking and deliberate self-harm Trends and patterns in Oxford, 19892002 Soc Psychiatry Psychiatr Epidemiol (2005) 40: 964-971

Haw, C., Hawton, K., Houston, K., et al (2001) Psychiatric and personality disorders in deliberate self-harm patients. $B M J, 178,48-54$.

Hawley, J.C. Littlechild, B. Sivakumaran, T. (2006) Structure and content of risk assessment proformas in mental healthcare. J Mental Health; 15:437-48.

Hawton, K. Fagg, J. (1988) Suicide, and other causes of death, following attempted suicide. British Journal of Psychiatry; 152:359-66.

Hawton, K. (2007) Psychiatric assessment and management of deliberate selfpoisoning patients. Medicine 35:10

Hawton, K. (2012) Self-harm and suicide in adolescents. The Lancet, Volume 379, Issue 9834, P. 2373-2382, doi:10.1016/S0140-6736(12) 60322-5

Hawton, K. Fagg, J. (1988) Suicide, and other causes of death, following attempted suicide. British Journal of Psychiatry; 152:359-66.

Hawton, K. Harris, L (2011) Deliberate self-harm in rural and urban regions: A comparative study of prevalence and patient characteristics. Social Science \& Medicine 73 (2011) 274-281

Hawton, K. Zahl, D. \& Weatherall, R. (2003). Suicide following deliberate selfharm: Long term follow-up of patients who presented to a general hospital. British Journal of Psychiatry, 182, 537-542.

Hawton, K., Rodham, K., Evans, E. (2006) By Their Own Young Hands. Deliberate Self harm and Suicidal Ideas in Adolescents. Jessica Kingsley Publishers. 
Health Professions Council Standards of Education and Training (2009) Health Care Professions Council: Standards of Education nd Training. Available from:

http://www.hpc-uk.org/aboutregistration/standards/sets/. [Accessed on 11/070/2016]

HCPC (2012) Health Care Professions Council: Standards of conduct, performance and ethics. Available from:

http://www.hpcuk.org/assets/documents/10003B6EStandardsofconduct,performance andethics.pdf [accessed on 15/04/2013]

HCPC (2012). Health \& Care Professions Council: Standards of proficiency Paramedics. Available from: http://www.hpc

uk.org/assets/documents/1000051CStandards_of_Proficiency_paramedics.pdf. [Accessed on: 22/05/2016]

HCPC (2017) consultation on the threshold level of qualification entry to the Register for paramedics. Available from:

https://www.hcpcuk.org/mediaandevents/news/index.asp?id=838 [accessed on $11 / 01 / 2018]$

HCPC (2018a.) update on consultation on the threshold level of qualification entry to the Register for paramedics. Available from

https://www.collegeofparamedics.co.uk/news/hcpc-consultation-on-changes-tothreshold-entry-level-to-the-register\#

HCPC (2018b.) Gender statistics of UK paramedics. Available from:

http://www.hpc-uk.org/aboutregistration/theregister/stats/ [accessed on 04/08/2018]

HSCIC (2015) Health \& Social Care Information Centre: Ambulance Services, England 2014-15. Available from:

http://www.hscic.gov.uk/catalogue/PUB17722/ambu-serv-eng-2014-2015-rep.pdf. [Accessed on 08/02/2015]

Health and Social Care Act (2012) Available from:

http://www.legislation.gov.uk/ukpga/2012/7/section/1/ enacted [Accessed on $12 / 06 / 2016]$ 
Health Professions Order (2001) Health Professions Order: Statutory Instrument No. 254 Available from: http://www.hpc-

uk.org/assets/documents/1000062120020254.htm [Accessed on 09/11/2014]

Heath, H. Cowley, S. (2004). Developing a grounded theory approach: a comparison of Glaser and Strauss. International Journal of Nursing Studies, 41, 141-150.

Heath, H. (2006) Exploring the influences and use of the literature during a grounded theory study. Journal of Research in Nursing. Vol 11, Issue 6, pp. $519-$ 528. 1, 2006 https://doi.org/10.1177/1744987106069338

HEFCE. REF 2014: Assessment framework and guidance on submissions 2011 [cited 201615 Feb]. Available from:

from: http://www.ref.ac.uk/media/ref/content/pub/assessmentframeworkandguidance onsubmissions/GOS\%20including\%20addendum.pdf. [accessed on 12/06/2017]

Hemsptead, K. (2006). The geography of self-injury: spatial patterns in attempted and completed suicide. Social Science \& Medicine, 62, 3186-3196.

Hirsch, J. (2006) A review of the literature on rural suicide. Risk and protective factors, incidence, and prevention. Crisis; 27(4):189-99.

Holden, S.J. Sheridan, R.D. Coffey, T.J. Scaramuzza, R.A. Diamantopoulos, P. (2007) Electromagnetic modelling of current flow in the heart from TASER devices and the risk of cardiac dysrhythmias. Phys Med Biol; 52:7193-209.

Ho, J.D. Miner, J.R. Lakireddy, D.R. Bultman, L.L. Heegaard, W.G. (2006) Cardiovascular and physiologic effects of conducted electrical weapon discharge in resting adults. Acad. Emerg. Med. 13 (2006) 589-595.

Holloway, I. (2005) Qualitative Research in Healthcare. Open University Press Hopkins, C. (20020 'But what about the really ill, poorly people?' (An ethnographic study into what it means to nurses on medical admissions units to have people who have harmed themselves as their patients). J Psychiatr Ment Health Nurs; 9: 147-54. 
Houston, K. Haw, C. Townsend, E. Hawton, K. (2003) General practitioner contacts with patients before and after deliberate self-harm. Br J Gen Pract. 53(490):365-370

House, J.B. Theyyunni, N. Barnosky, A.R. Fuhrel-Forbis, A.. Seeyave, D.M. Ambs, D. Fischer, J.P. Santen, S.A. (2015) Understanding ethical dilemmas in the emergency department: views from medical students' essays. J Emerg Med. 2015 Apr;48(4):492-8. doi: 10.1016/j.jemermed.2014.09.058.

Hughes, R. (1998) Considering vignette technique and its application to a study of drug injecting and HIV risk and safer behaviour. Sociology of Health and Illness, 20(3) 381- 400.

Human Rights Act (1998) Available from:

http://www.legislation.gov.uk/ukpga/1998/42 http://www. [Accessed on 22/02/2016]

Hume, M. Platt, S. (2007) Appropriate interventions for the prevention andmanagement of self-harm: a qualitative exploration of service-users' views. BMC Public Health; 7:9

Hunter, C. Chantler, K. Kapur N, (2013) Service user perspectives on psychosocial assessment following self-harm and its impact on further help-seeking: a qualitative study. J Affect Disord; 145:315-23.

Huriash, L. J. (2001,) Police team to focus on the mentally ill. Sun-Sentinel, pp.5B. October 15

IHCD (2000) Institute of Health and Care Development ambulance personnel awards: training and common core syllabus for ambulance personnel. London: Ambulance Service Association, 2000.

IHCD (2003) Institute of Health and Care Development Paramedic training rules and regulations. Bristol: Institute of Health and Care Development, 2003.

Ilgen, M.A. Burnette, M.L. Conner, K. R. (2010) The association between violence and lifetime suicidal thoughts and behaviours in individuals treated for substance use disorders. Addict.Behav. 35(2),111-115. 
Iles, V. (2011) Why Reforming the NHS Doesn't Work: The Importance of Understanding How Good People Offer Bad Care. Really Learning, London.

Imison, C. Sonola, L. Honeyman, M. Ross, S (2014). The reconfiguration of clinical services: what is the evidence? London: The King's Fund. Available from:

www.kingsfund.org.uk/publications/reconfiguration-clinical-services [accessed on 2 November 2015]

INVENT (2014) Development of a pre-hospital mental health model-of-care for application and testing in the Support and Assessment for Emergency Referral (SAFER 4) trial. Available from:

file:///C:/Users/bilbo/Downloads/INVENT\%20Final\%20Report\%20July\%202013\%2 0(2).pdf [Accessed on 16/092015]

IPCC (2015) Independent Police Complaints Commission: Police Custody as a "Place of Safety": Examining the Use of Section 136 of the Mental Health Act 1983. Available from:

https://www.ipcc.gov.uk/sites/default/files/Documents/section_136.pdf [Accessed on $12 / 06 / 2015]$

IPCC (2008) Deaths during or following police contact: Statistics for England and Wales 2007/08. England and Wales: Independent Police Complaints Commission. Available from:

https://www.policeconduct.gov.uk/sites/default/files/Documents/statistics/deaths_dur ing_following_police_contact_201718.pdf. [Accessed on 12/10/2017]

Jacobs, I. Nadkarni, V. (2004) The ILCOR Task Force on Cardiac Arrest and Cardiopulmonary Resuscitation Outcomes, Bahr J, Berg RA, Billi JE et al.: Cardiac Arrest and Cardiopulmonary Resuscitation Outcome Reports: Update and Simplification of the Utstein Templates for Resuscitation Registries: A Statement for Healthcare Professionals From a Task Force of the International Liaison Committee on Resuscitation (American Heart Association, European Resuscitation Council, Australian Resuscitation Council, New Zealand Resuscitation Council, Heart and Stroke Foundation of Canada, Inter American Heart Foundation, Resuscitation Councils of Southern Africa). Circulation 2004, 110: 3385-3397. 
Jasper, M. (1994) Issues in phenomenology for researchers of nursing. Journal of Advanced Nursing. Volume 19, Issue 2 February 1994. P 309-314

Jeglic, E. L. Vanderhoff, H. A. Donovick, P. J. (2005). The function of self-harm behaviour in a forensic population. International Journal of Offender Therapy and Comparative Criminology, 49(2), 131-142.

Jensen (2010) Paramedic clinical decision making. Available from:

http://www.australianparamedicalcollege.com.au/learning-material/adv-diphealthcare/paramedic-clinical-decision-making.pdf. [Accessed on 17/12/2017]

Jensen, L.A. Allen, M.N. (1996) Meta-synthesis of qualitative findings. Qual Health Res. 6: 553-60.

Johnson, B.R. (1997) Examining the validity structure of qualitative research. Education, 118 (3): 282-292 Jones and Bartlett publishers inc.

Johnson, M. Long, T. (2007) Research Ethics in the Real World: Issues and Solutions for Health and Social Care. Churchill Livingstone Elsevier, Edinburgh.

Joinson, C. (1992). Coping with compassion fatigue. Nursing, 22, 118-121.

Jones (2008) Diagnostic overshadowing: Worse physical health care for people with mental illness. Acta Psychiatr Scand: 118: 169-171

JRCALC (2000) The Joint Royal Colleges and Ambulance Liaison Committee and The Ambulance Service Association. The future role and education of paramedic ambulance personnel (emerging concepts). London: JCALC/ASA, 2000.

JRCALC (2004) Joint Royal Colleges Ambulance Liaison Committee Clinical Practice Guidelines. Available From: http://www.nelhec.warwick.ac.uk/JRCALC_Guidelines_v3_2004.pdf [Accessed on 22/06/2015]

JRCALC (2013) Joint Royal Colleges Ambulance Liaison Committee Clinical Practice Guidelines. Available From: www.jrcalc.org.uk/guidelines/current-edition/ [Accessed on 22/06/2015] 
JRCALC (2015) A brief history of ambulance services. Available from:

http://www2.warwick.ac.uk/fac/med/research/hsri/emergencycare/prehospitalcare/jrc alcstakeholderwebsite/history-copy/ [Accessed on 27/12/2015]

JRCALC (2016) Joint Royal Colleges Ambulance Liaison Committee Clinical Practice Guidelines. Available From: www.jrcalc.org.uk/guidelines/ [Accessed on $22 / 06 / 2016]$

Judd, F. Cooper, A.M. Fraser, C. Davis, J. (2006) Rural suicide-people or place effects? Aust N Z J Psychiatry;40(3):208-16.

Kapur, N (2006). Self-harm in the general hospital. Clinical Medicine 6, 529-532.

Kapur, N. Murphy, E. Cooper, J. Bergen, H. Hawton, K. Simkin, S. Casey, D. Horrocks, J. Lilley, R. Noble, R. Owens, D. (2008). Psychosocial assessment following self-harm: results from the Multi Centre Monitoring of Self-Harm Project. J Affect Disord 106, 285-293.

Kapusta, N. Zorman, A. Etzerdorfer, E. Ponocny-Seliger, E. Jandl-Jager, E. \& Sonneck, G. (2008). Rural-urban differences in Austrian suicides. Social Psychiatry and Psychiatric Epidemiology. 43, 311e318.

Kattimani, S. Sarkar, S. Rajkumar, R. Menon, V. (2015) Stressful life events, hopelessness, and coping strategies among impulsive suicide attempters. J. Neurosci. Rural Pract. 6, 171 176, http://dx.doi:10.4103/0976-3147.153222.

\section{Keenan v UK (2001) 33 EHRR 913}

Keogh, B. (2013) High quality care for all, now and for future generations: transforming urgent and emergency care services in England-urgent and emergency care review end of phase 1 report. 1-30. NHS England, 2013. Available from: http://www.nhs.uk/NHSEngland/keoghreview/Documents/UECR.Ph1Report.Append ix\%201.EvBase.FV.pdf. [Accessed on 27/01/2016]

Kerfoot, K.E. Rosenheck, R.A. Petrakis, I.L. Schwartz, M.S. Keefe, R.S.E. McEvoy, J.P. 
Kilner, T. (2004) Desirable attributes of ambulance technician, paramedic, and clinical supervisor: findings from a Delphi study. Emerg Med J; 21:374-8

King's College London and Digital Science. (2015). The nature, scale and beneficiaries of research impact: An initial analysis of Research Excellence Framework (REF) 2014 impact case studies. London: King's College London.

Kirkby, H.M. Roberts, L.M. (2012) Inappropriate 999 calls: an online pilot survey. Emerg Med J ;29:141-146. doi:10.1136/emj.2010.092346

Kisely, S. Campbell, L. A. Peddle, S. (2010). A controlled before-and-after evaluation of a mobile crisis partnership between mental health and police services in Nova Scotia. Canadian Journal of Psychiatry, 55, 662-668.

Klonsky, E.D. Muehlenkamp, J.J. (2007) Self-injury: a research review for the practitioner. Journal of Clinical Psychology. 63,1045-1056.

Knowlton, A. Weir, B.W. Hughes, B. S. Southerland, B. Schultz, W. Sarpatwari, R. Wissow, L. Links, J. Fields, J. McWilliams, J. BA, Wade, Gaasch. (2013) Patient demographic and health factors associated with frequent use of emergency medical services in a mid-sized city. Acad Emerg Med. November ; 20(11): 1101-1111. doi:10.1111/acem

Kolb, D.A. (1984) The experiential learning theory of development. In: Kolb, D.A. (Ed.), Experiential Learning: Experience as the Source of Learning and Development. Prentice Hall, Englewood Cliffs, NJ, pp. 132e160

Koopmans, E. Wagner, S.L. Schmidt, G. Harder, H.G. (2017) Emergency Response Services Suicide: A Crisis in Canada? July 2017 Journal of Loss and Trauma. 22(11) DOI 10.1080/15325024.2017.1360589

Krauss, S. E. (2005) Research paradigms and meaning making: A primer. The Qualitative Report. 10(4):758-770. Available from http://www.nova.edu/ssss/QR/QR 10-4/krauss.pdf. [Accessed on 22/01/2015] 
Kumar, P.N.S. Rajmohan, V. Sushil, K. (2013) An exploratory analysis of personality factors contributed to suicide attempts. Indian J. Psychol. Med. 35, 378384, http://dx.doi:10.4103/0253-7176.122231.

LaCalle, E. Rabin, E. (2010) Frequent users of emergency departments: the myths, the data, and the policy implications. Ann Emerg Med; 56:42-8.

Laing, R., Halsey, R. Donohue, D. (2009). Application of a model for the development of a mental health service delivery collaboration between police and the health service. Issues in Mental Health Nursing, 30, 337-341.

Lamb, H. R. Weinberger, L. E. DeCuir, W. J. Jr (2002). The police and mental health. Psychiatric Services, 53, 1266-1271.

Lanes, E. (2009). Identification of risk factors for self-injurious behaviour in male prisoners. Journal of Forensic Sciences, 54(3), 692-698.

Law BM, Shek DT. A 6-year longitudinal study of self-harm and suicidal behaviors among Chinese adolescents in Hong Kong. J Pediatr Adolesc Gynecol. 2016;29 (Suppl 1): S38-48.

Lee, K.H. Davenport, L. (2006) Can case management interventions reduce the number of emergency department visits by frequent users? Health Care Management. April-June 25(2):255-9

Lemonick, D.M. (2009)C ontroversies in Prehospital Care. American Journal of Clinical Medicine: 6:1

Lempp, H. Seale, C. (2004) The hidden curriculum in undergraduate medical education: qualitative study of medical students' perceptions of teaching. $B M J$; 329:770-3.

Lendrum, K. Wilson, S. Cooke, M.W. (2000) Does the training of ambulance personnel match the workload seen? Pre-hospital Immediate Care; 4:7-10.

Levin, K. Leyland, A. (2005). Urban/rural inequalities in suicide in Scotland, 19811999. Social Science \& Medicine. 60: 2877-2890. 
Levine, S.D. Sloane, C. Chan, T. Dunford, J. Vilke, G (2007) Cardiac monitoring of human subjects exposed to the TASER. J. Emerg. Med. 33: 113-117.

Li, Y.M (2007) Deliberate self-harm and relationship to alcohol use at an emergency department in eastern Taiwan. Kaohsiung J Med Sci; 23: 247-253

Lilley, R. Owens, D. Horrocks, J. (2008) Hospital care and repetition following selfharm: multicentre comparison of self-poisoning and self injury. BMJ. 192: 440-445.

Lincoln, Y. S. Guba, E. G. (2000). Paradigmatic controversies, contradictions, and emerging influences. In N. Denzin and Y. Lincoln (eds.), Handbook of Qualitative Research (2 ${ }^{\text {nd }}$ ed., pp. 163-188). Thousand Oaks, CA: Sage

Lincoln, Y. S. Guba, E. G. (2005). Paradigmatic controversies, contradictions, and emerging influences. In N. Denzin and Y. Lincoln (eds.), Handbook of Qualitative Research (2nd ed., pp. 163-188). Thousand Oaks, CA: Sage.

Lindner, T.W. Soreide, E. Nilsen, O.B. Torunn, M.W. Lossius, H.M. (2011) Good outcome in every fourth resuscitation attempt isachievable-An Utstein template report from the Stavanger region. Resuscitation 2011; 82:1508-13

Link, B.G., Phelan, J.C., 2006. Stigma and its public health implications. Lancet. $367,528-529$

Linsley, K.R. Schapira, K. Kelly, T.P. (2001) Open verdict v. suicide-importance to research. Br J Psychiatry. 178:465-8.

Lloyd-Richardson, E.E. Perrine, N. Dierker, L. Kelley, M.L. (2007) Characteristics and functions of non-suicidal self-injury in a community sample of adolescents. Psychol Med. ;37(08):1183-1192.

LSE Public Policy Group (2011). Maximizing the impacts of your research: A handbook for social scientists. Available from:

http://www.lse.ac.uk/government/research/resgroups/LSEPublicPolicy/Docs/LSE_I mpact_Handbook_April_2011.pdf London: LSE; 2011. [accessed on 04/04/2018] 
Luck, M. Townsend, R. (2012) Applied Paramedic Law and Ethics. Elsevier: Sydney.

Ma, C.M. Shek, D.T (2014) Prevalence and psychosocial correlates of after-school activities among Chinese adolescents in Hong ong. Front Public Health; 2:159

Machen, I. Dickenson, I. Williams, J. Widiatmoko, D. Kendal, S. (2007) Nurses and paramedics in partnership: Perceptions of a new response to low-priority ambulance calls. Accident and Emergency Nursing (2007) 15, 185-192

Macintyre, S. Maciver, S. Soomans, A. (1993). Area, class and health: should we be focusing in places or people? Journal of Social Policy. 22, 213-234

Mackay, N. Barrowclough, C. (2005) Accident and emergency staff's perceptions of deliberate self-harm: attributions, emotions and willingness to help. British Journal of Clinical Psychology. 44, 255-267.

Maguire BJ. Violence against ambulance personnel: a retrospective cohort study of national data from Safe Work Australia. Public Health Res Pract. 2018;28(1):e28011805. https://doi. org/10.17061/phrp28011805

Mallia, P. (2003) Biomedical ethics: The basic principles. Student BMJ. Vol. 11 MAY 2003

Malone R. Heavy users of emergency services: social construction of a policy problem. Soc Sci Med. 40:469-77

Marris, R.W. (2002) Suicide. Lancet, 360: 31997326.

Martha, M. Scheckel, M. Nelson, K.A. (2014) an interpretive study of nursing students' experiences of caring for suicidal persons. Journal of Professional Nursing. vol 30, no. 5: pp 426-435

Mason, S.M. (2014) Frequent attendance at the emergency department is a symptom but not a disease. Emerg Med J; 31:524-25.

Matheson, F. I. Creatore, M. I. Gozdyra, P. Moineddin, R. Rourke, S. B. \& Glazier, R. H. (2005). Brief Reports: Assessment of Police Calls for Suicidal Behaviour in a 
Concentrated Urban Setting. Psychiatric Services, 56(12), 1606-1609. doi: 10.1176/appi.ps.56.12.1606

Mavrogiorgou, P., Brüne, M., \& Juckel, G. (2011). The management of psychiatric emergencies. Deutsches Ärzteblatt International. 108(13), 222-230.

Maytum, J. Heiman, M. Garwick, A. (2004). Compassion fatigue and burnout in nurses who work with children with chronic conditions and their families. Journal of Pediatric Health Care, 18, 171-179.

McAllister, M. Higson, D. McIntosh, W. O'Leary, S. Hargreaves, L. Murrell, L. Mullen, V. Lovell, F. Kearney, J. Sammon, D. Woelders, S. Adams, T. DaviesCotter, D. Wilson, J. O’Brien, J. (2001) Dissociative Identity Disorder and the nurse patient relationship in the acute care setting: An action research project. Australian and New Zealand Journal of Mental Health Nursing. 10, 20-32

McAllister, M. Creedy, D. Moyle, W. Farrugia, C. (2002). Nurses' attitudes towards clients who self harm. Journal of Advanced Nursing, 40, 578-586.

McAllister, M. Zimmer-Gembeck, M. Moyle, W. Billett, S. (2008) Working effectively with clients who self-injure using a solution focused approach. International Emergency Nursing. 16:272-279.

McCann, T. Clark, E. Mc Connachie, S. Harvey, I. (2006) Accident and emergency nurses' attitudes towards patients who self-harm. Accid Emerg Nurs; 14:4-10.

McCarthy L, Gijbels H. An examination of emergency department nurses' attitudes towards deliberate self-harm in an Irish teaching hospital. Int Emerg Nurs; 18:29-35.

McCreaddie, M. Payne, S (2010) Evolving Grounded Theory Methodology: Towards a discursive approach. International Journal of Nursing Studies. 47: 781-793

McGhee, G. Marland, G.R. Atkinson, J. (2007) Grounded theory research: literature reviewing and reflexivity. Journal of Advanced Nursing. 60, 3, 334-342. 
McHale, J., Felton, A., 2010. Self-harm: what's the problem? A literature review of the factors affecting attitudes towards self-harm. J. Psychiatr. Ment. Health Nurs. 17 (8), 732-740.

McManus, S. Meltzer, H. Brugha, T. (2009) Adult Psychiatric Morbidity in England, 2007. Results of a Household Survey. Health and Social Information Centre, Social Care Statistics.

Mental Capacities Act (2005) HMSO. London available from:

htp://www.legislation.gov.uk/ukpga/2005/9/contents [Accessed on 27/03/2014]

Mental Health Code of Practice (2015) The Stationery Office. Available from: https://www.gov.uk/government/publications/code-of-practice-mental-health-act1983 [Accessed on 22/02/2016]

Mental Health Act (Aus) (2000) Australian Mental Health Act. Available from: https://www.legislation.qld.gov.au/LEGISLTN/ACTS/2000/00AC016.pdf [Accessed on 03/09/2017]

Mental Health Act. (2007) Amendments to Mental Health Act 1983. HMSO London. Available from:

http://www.legislation.gov.uk/ukpga/2007/12/pdfs/ukpga_20070012_en.pdf; [Accessed on 03/09/2017]

Mental Health Code of Practice (2015) The Stationery Office. Available from: https://www.gov.uk/government/publications/code-of-practice-mental-health-act1983 [Accessed on 22/02/2016]

Mental Health Crisis Care Concordat (2014) Improving outcomes for people experiencing mental health crisis. HMSO. Available from: www.gov.uk/dh. [Accessed on 13/06/2015]

Mental health foundation (2006) National inquiry National Inquiry into Self-harm among Young People: Truth Hurts: London. Mental health foundation. Available from: www.mentalhealth.org.uk [Accessed on 12/05/2014] 
Miles, M. B. \& Huberman, A. M. (1984). Qualitative data analysis: A source book of new methods. Beverly Hills, CA: Sage.

Mills, J. Bonner, A. Karen, F (2006). Adopting a constructivist approach to grounded theory: Implications for research design. International Journal of Nursing Practice. $12(1), 8-13$.

MIND (2004) Understanding self harm. Available from:

http://www.mind.org.ukllnformationhl300kletsiUnderstafldiflg!UflderStafldlflg+SCl f-harm.htrn [Accessed on12/07/20014]

Ministry of Health (1966a) Scottish Home and Health Department. Report by the working party on ambulance training and equipment: part 1-training. London: HMSO, 1966.

Ministry of Health (1966b) Scottish Home and Health Department. Report by the working party on ambulance training and equipment: part 2-equipment and vehicles. London: HMSO, 1967.

Moed, H. F. (2005). Citation analysis in research evaluation. Dordrecht: Springer.

Moher D, Liberati A, Tetzlaff J, Altman DG, The PRISMA Group (2009). Preferred Reporting Items for Systematic Reviews and Meta-Analyses: The PRISMA Statement. PLoS Med 6(6): e1000097. doi:10.1371/journal.pmed1000097

Morgan, C. Thayre, S. (2016) Frequent caller management SWASFT. Emerg Med $J ; 33:$ e3.

Morris T, Mason, S.M. Moulton, C. O'Keeffe, C. (2017) Calculating the proportion of avoidable attendances at UK emergency departments: analysis of the Royal College of Emergency Medicine's Sentinel Site Survey data. Emerg Med J;0:1-6. doi:10.1136/emermed-2017-206846

MRC (2005) Medical Research Council position statement on research regulation and ethics. Available from: http://www.mrc.ac.uk/Utilities/Documentrecord/index.htm?d=MRC002462 [Accessed on 16/04/1 
Munhall, P.L. (2001) Nursing Research: A Qualitative Perspective. Jones and Bartlett publishers inc. 3rd Edition. London.

Munhall, P.L (2008) The SAGE Encyclopaedia of Qualitative Research Methods. SAGE Publications, Inc. Thousand Oaks

Munjal, K.G. Silverman, R.A. Freese, J. Braun, J.D. Kaufman B.J. Isaacs, D. Werner, A. Webber, M. Hall, C.B. Prezant, D.J. (2011) Utilization of emergency medical services in a large urban area: description of call types and temporal trends. Prehosp Emerg Care. 2011; 15(3):371-380.

Murphy, A. (1998) 'Inappropriate' attenders at accident and emergency departments I: definition, incidence and reasons for attendance. Fam Pract;15:23-32.

Murphy, E. Dingwall, R (2007) Informed consent, anticipatory regulation and ethnographic practice. Social Science and Medicine. 65:2223-2234.

Murphy, G (2000) Psychiatric aspects of suicidal behaviour: substance abuse. In: Hawton K, Van Heeringen K (eds) The International handbook of suicide and attempted suicide. Wiley, Chichester, pp 135-146

NAO (2010) National Audit Office: Major Trauma in England. Available from: https://www.nao.org.uk/wp-content/uploads/2010/02/0910213.pdf [Accessed on 28/03/2016]

National Audit Office (2017) National Audit Office (Eng) NHS Ambulance Services. Available from: https://www.nao.org.uk/wp-content/uploads/2017/01/NHSAmbulance-Services.pdfNational [accessed on 01/10/2017]

National Collaborating Centre for Mental Health (2004) in: RCP (2010) Royal College of Psychiatrists' report on self harm, suicide, and risk. Available from: http://www.rcpsych.ac.uk/files/pdfversion/cr158.pdf [Accessed on 02/03/2015] National Health Service Executive (1998) A First Class Service, Health Service Circular 1998/113. London: NHS Executive. 
National Health Service Executive (1999) Modernisation of Ambulance Services. Health Service Circular 1999/091. London: NHS Executive.

NHS Executive (2000) National Service Framework for Coronary Heart Disease: Modern Standards and Service Models. London: NHS Executive.

National Institute for Mental Illness in England (2015) Crisis resolution and home treatment. Available from: http://bcu.ac.uk/Download/Asset/50450b3b-d18c-4900b985-5218b3b13afb. [Accessed on 12/03/2016]

National Voices (2013) National voices' vision for person centred coordinated care. Available from: https://www.nationalvoices.org.uk/sites/default/files/public/nationalvoices-narrative-leaflet.pdf [accessed on 28/05/2017]

NCCMH (2004) National Collaborating Centre for Mental Health Self-harm: The Short-term Physical and Psychological Management and Secondary Prevention of Self-harm in Primary and Secondary Care. National Institute for Health and Clinical Excellence. Available from: http://www.nice.org.uk/nicemedia/live/10946/29424/29424.pdf [Accessed on 20/04/2011]

Neff, K.D., 2003. The development and validation of a scale to measure selfcompassion. Self and Identity; 2, 223-250.

Nest (2018) Fun facts about EMT's. Available form: https://woman.thenest.com/funemts-14156.html. [accessed on 04/08/2018]

Neuman, W. L. (2003). Social Research Methods: Qualitative and Quantitative Approaches ( $5^{\text {th }}$ ed.). Boston: Allyn and Bacon.

Neville, L. Haigh, C. (2003) A response to Martin Johnson's Editorial 'Research ethics and education: A consequentialist view'. Nurse Education Today 23: 549-553 NHS Confederation (2007b) NHS Ambulance Services . . More Than Just Patient Transport. London: NHS Confederation. 
NHS Confederation (2015) Urgent and Emergency Care Forum Rip off the sticking plaster now Enabling the local implementation of sustainable urgent and emergency care models in 2015/16. Available from:

http://nhsconfed.org/ /media/Confederation/Files/Publications/Documents/rip_off_st icking_plaster_now0515.pdf [Accessed on 27/12/2015]

NHS England (2015) Ambulance-quality-indicators. Available from:

Www.england.nhs.uk/statistics/stasistical-work-areas/ambulance-quality-indicators/. [Accessed on 21/06/2015]

NHSTD (1991) National Health Service Training Directorate. Ambulance Service Paramedic Training. Bristol: NHSTD, 1991

NICE (2004) The short-term physical and psychological management and secondary prevention of self-harm in primary and secondary care. Available from www.nice.org.uk [Accessed on 2/01/2014]

NIHR (2016) Themed Review Research for Ambulance Services: Care at the scene. Steering group member. Available from: http://www.dc.nihr.ac.uk/themedreviews/care-at-the-scene.htm. [Accessed on 01/10/2017]

NISCHR (2011) National Institute of Social Care and Health Research. Available from:

http://www.wales.nhs.uk/sites3/Documents/952/Gaining\%20NHS\%20Research\%20 Permission\%20in\%20Wales\%20-\%20April\%202013\%20-\%20secure.pdf. [accessed on $19 / 04 / 2018]$

Noblit, G.W. Hare, R. D. (1988) Meta-Ethnography: Synthesizing Qualitative Studies. London: Sage; 1988

Nock, M.K. (2009) Why Do People Hurt Themselves? New Insights Into the Nature and Functions of Self-Injury. Association for Psychological Science. Volume 18 Number 2

Norheim, B. Grimholt, T.K. Ekeberg, O. (2013) Attitudes towards suicidal behaviour in outpatients clinics among mental health professionals in Oslo. BMC Psychiatry.13:90 
NRES (2015) Guidance for applicants Available from:

http://www.nres.nhs.uk/applications/ [accessed on 16/09/2015]

NSFMH (1999) National Service Framework Mental Health. Available from: https://www.gov.uk/government/uploads/system/uploads/attachment_data/file/19805 1/National_Service_Framework_for_Mental_Health.pdf [Accessed on 27/03/2016]

Nurok, M. Henckes, N. (2009) Between professional values and the social valuation of patients: the Fluctuating economy of pre-hospital emergency work. Soc Sci Med 2009;68: 504-10.

NVIVO (2015) NVIVO Version 10: QSR. Available from:

http://www.qsrinternational.com/nvivo-product. [accessed on 27/04/2017]

O’Connor, R. C. Rasmussen, S., Miles, J. (2009) Self-harm in adolescents: self report survey in schools in Scotland. BMJ, 194, 68-72.

O’Hara, R. Johnson, M. Siriwardena, N. Weyman, A. Turner, J. Shaw, D. Mortimer, P. Newman, C. Hirst, E. Storey, M. Mason, S. Quinn, T. Shewan, J. (2015) A qualitative study of systemic influences on paramedic decision making: care transitions and patient safety. Journal of Health Services Research \& Policy. 20 Suppl. 1

O’Neill, S. Colette, V. Corry. Murphy, S. Brady, S. Bunting, B, P. (2014) Characteristics of deaths by suicide in Northern Ireland from 2005 to 2011 and use of health services prior to death. Journal of AffectiveDisorders. 168; 466-471

Obafunwa, J. Busuttil, A. (1994). A review of completed suicides in the Lothian and Borders region of Scotland (1987e1991). Social Psychiatry and Psychiatric Epidemiology. 29, 100-106.

Oginska-Bulik, N. Kaflik-Piero’g, M. (2009) Occupational stress in rescue services. qo’d_z, Poland: WSH-E; 2009.

Okin, R.L. Boccellari, A. Azocar, F. (2000) The effects of clinical case management on hospital service use among ED frequent users. Am J Emerg Med; 18:603-8. 
Oliffea, J.L. Ogrodniczukc, J.S. Bottorff, J.L. Johnsona, J.L. Hoyaka, K. (2012) "You feel like you can't live anymore": Suicide from the perspectives of Canadian men who experience depression. Social Science \& Medicine. 74 (2012) 506-514

ONS (2014 a) Reported road casualties in Great Britain: main results 2013. Available from: https://www.gov.uk/government/statistics/reported-road-casualtiesin-great-britain-main-results-2013 [Accessed on 13/06/2015]

ONS (2014 b) Suicides in the United Kingdom, 2013 Registrations. Available from: http://www.ons.gov.uk/ons/dcp171778_395145.pdf [Accessed on 13/06/2015]

Otahbachi, M. Cevik, C. Bagdure, S. \& Nugent, K. (2010). Excited delirium, restraints and unexpected death: A review of pathogenesis. American Journal of Forensic Medicine and Pathology. 31, 107-112.

Ouzouni C, Nakakis K. (2013) Nurses' attitudes towards attempted suicide. Health Science Journal. 2013;7 (1):119-134

Owens, D. Horrocks, J. House, A. (2002) Fatal and non-fatal repetition of self- harm. British Journal of Psychiatry. 180 (3) 193-199.

Owens, D, Kelley, R, Munyombwe, T et al. (7 more authors) (2015) Switching methods of self-harm at repeat episodes: Findings from a multicentre cohort study. Journal of Affective Disorders, 180. pp. 44-51. ISSN 0165-0327 https://doi.org/10.1016/j.jad.2015.03.051

Pages, F. Arvers, P. Hassler, C. Choquet, M. (2004). What are the characteristics of adolescent hospitalized suicide attempters? European Child \& Adolescent Psychiatry, 13, 151-158.

Pajonk, F.G. Bartels, H.H. Biberthaler, P. Bregenzer, T. Moecke, H. (2001) Psychiatric emergencies in preclinical emergency service: incidence, treatment and evaluation by emergency physicians and staff. Der Nervenarz. 72(9):685-692

Palazzo, F. Warner, O.J. Harron, M (1998) Misuse of the London ambulance service: how much and why? J Accid Emerg Med; 15:368-70 
Parsons, T., 1958. Definitions of health and illness in the light of American values. In: Jaco, E.G. (Ed.), Patients, Physicians and Illness: Sourcebook in Behavioral Science and Medicine. The Free Press, Glencoe, pp. 165-187

Paton F, Wright K, Ayre N, Dare C, Johnson S, Lloyd-Evans B, (2016) Improving outcomes for people in mental health crisis: a rapid synthesis of the evidence for available models of care. Health Technol Assess 2016;20(3).

Peers, I. (1996) Statistical analysis for education and psychology researchers. Bristol, PA: Falmer Press

Pels, P. (1999) Professions of duplexity, a prehistory of ethical codes in anthropology. Current Anthropology 1999, 40(2):101-136.

Pennycook, A. Makower, R. Morrison, W. (1991) Use of the emergency ambulance service to an inner city accident and emergency department a comparison of general practitioner and '999' calls. J R Soc Med; 84:726-7.

Penterman, B. Nijman, H. (2011). Assessing aggression risks in patients of the ambulatory health crisis team. Community Mental Health, 47, 463-471.

Pines JM, Asplin BR, Kaji AH, Lowe RA, Magid DJ, Raven M, (2011) Frequent users of emergency department services: gaps in knowledge and a proposed research agenda. Acad Emerg Med. 2011;18: e64-e69. pmid:21676051

Platt, S. Hawton, K. (2000) Suicidal behaviour and the labour market. In: Hawton K, Van Heeringen K, eds. The international handbook of suicide and attempted suicide. Chichester: Wiley, 2000.

Police and Crime Act (2017) Available from: http://www.legislation.gov.uk/ukpga/2017/3/part/4/chapter/4/enacted [accessed on 23/2/2018]

Polit, D.F. Beck, C.T. (2004) Nursing Research: Principles and Methods, seventh ed. Lippincott Williams and Wilkins, Philadelphia 
Pollock, K. (2012) Procedure versus process: ethical paradigms and the conduct of qualitative research. BMC Medical Ethics, 13:25

Pope, D. Fernandes, C.M. Bouthillette, F et al. (2000) Frequent users of the emergency department: a program to improve care and reduce visits. CMAJ;162:1017-20.

Porter, A. Snooks, H. Youren, A. Gaze, S. Whitfield, R. Rapport, F. Woollard, M. (2007) 'Should I stay or should I go?' Deciding whether to go to hospital after a 999 call. Journal of Health Services Research \& Policy. Vol 12 Suppl 1, 2007: 32-38

Porter, R.B. (2010) Nurse managers' moral distress in the context of the hospital ethical climate. Available from:

https://pdfs.semanticscholar.org/67d1/2edf5d6c90c217e3f8570c7f74b2835d82a5.pdf [accessed on 10/12/2017]

Potton, M. Q. (1990) Qualitative Evaluation and Research Methods. $2^{\text {nd }}$ Edition. London, Sage

Pozner, C.R. Zane, R. Nelson, S.J. Levine, M. (2004) International EMS Systems: The United States: past, present, and future. Resuscitation 60 (2004) 239-244

Quinlivan, L. Cooper, J. Steeg, S. Davies, L. Hawton, K. Gunnell, D. Kapur, N. (2014) Scales for predicting risk following self-harm: an observational study in 32 hospitals in England. BMJ Open; 4:e004732. doi:10.1136/bmjopen-2013-004732

Quinlivan, L. Cooper, J. Meehan, D. Longson, D. Potokar J. Hulme, Marsden, J. Brand, F. Lang, K. Riseborough, E. Page, L. Metcalfe, C. Davie, L. O'Connor, R. Hawton , K. T. Gunnell, D. Kapur, N. (2017) Predictive accuracy of risk scales following self-harm: multicentre, prospective cohort study. The British Journal of Psychiatry Jun 2017, 210 (6) 429-436; DOI: 10.1192/bjp.bp.116.189993

Rabone and another v Pennine Care NHS Foundation Trust [2012] UKSC 2. Rasic, D. Belik, S. Elias, B. Katz, L. Enns, M. Sareen, J. (2009) Spirituality, religion and suicidal behavior in a nationally representative sample. Journal of Affective Disorders 114 (1-3), 32-40. 
Rasic, D. Belik, S. Elias, B. Katz, L. Enns, M. Sareen, J. (2009) Spirituality, religion and suicidal behavior in a nationally representative sample. Journal of Affective Disorders 114 (1-3), 32-40.

RCPsych (2006) Royal College of Psychiatrists: Better Services for People who SelfHarm Quality Standards for Healthcare Professionals. Available from: http://www.rcpsych.ac.uk/cru/auditSelfHarm.htm. [Accessed on 12/05/2014] RCPsych (2010) Psychiatrists' report on selfharm, suicide, and risk. Available from: http://rcpsych.ac.uk/ publications/collegereports/cr/cr158.aspx [Accessed on 02/03/2014]

RCPsych (2013) Royal College of Psychiatrists: Whole-person care: from rhetoric to reality Achieving parity between mental and physical health. Available from: http://www.rcpsych.ac.uk/pdf/OP88.pdf [Accessed on 28/02/2016]

Re T (adult: Refusal of Medical Treatment) [1993] Fam 95 at 103 (Donaldson LJ) Reason, J. T. (1990). Human error. Cambridge, England: Cambridge University Press.

Rees, N, Rapport, F, Thomas, G. John, A, Snooks,H. (2014) Perceptions of Paramedic and Emergency Care Workers of those who Self Harm: A Systematic Review of the Quantitative Literature. Journal of Psychosomatic Research. 77(6): 449-56. doi: 10.1016/j.jpsychores.2014.09.006.

Rees, N. Rapport, F. Snooks, H. (2015) Perceptions of paramedics and emergency staff about the care they provide to people who Self Harm: Constructivist Metasynthesis of the qualitative literature. Journal of Psychosomatic Research. 78(6):529-35. doi: 10.1016/j.jpsychores.2015.03.007.

Rees, N. Rapport, Snooks, H. John, A. (2017) How do emergency ambulance paramedics view the care they provide to people who self harm?: Ways and Means. In press: accepted for publication. International Journal of Law \& Psychiatry. 50:6167. DOI 10.1016/j.ijlp.2016.05.010 
Rees, N. Whitfield, R (2005) Violence and Aggression directed towards Ambulance Services personnel: Guidance through application of behavioural sciences.

Ambulance UK. Vol 20:3. P. 267-176

Reuland, M. Schwarzfeld, M. Draper, L. (2009) Law Enforcement Responses to People with Mental Illnesses: A Guide to Research Informed Policy and Practice. Council for State Governments Justice Center; New York, NY: 2009.

Rhodes, A. Bethell, J. Spence, J. Links, P. Streiner, D. Jaakkimainen, R. (2008) Agesex differences in medicinal self-poisonings: a population-based study of deliberate intent and severity. Soc Psychiatry Psychiatr Epidemiol; 43(8):642-52.

Richards, J. Ferrall, S. (1999) Inappropriate use of emergency medical services transport: comparison of provider and patient perspectives. Acad Emerg Med; 6:1420.

Rinke, M.L. Dietrich, E. Kodeck, T, (2012) Operation care: a pilot case management intervention for frequent emergency medical system users. Am J Emerg Med; $30: 352-7$.

Rittel, H.W.J. Webber, M.W. (1973) Dilemmas in a general theory of planning. Policy Sciences; 4:155-69.

Roberts, L. Henderson, J (2009) Paramedic perceptions of their role, education, training and working relationships when attending cases of mental illness. Journal of Emergency Primary Health Care (JEPHC), 7: 3.

Robinson-Steel, I. (2004) "Reforming Emergency Care": the ambulance impact. A personal view. Emerg Med J; 21:207-211 doi:10.1136/emj.2002.001669

Robson, C. (2002). Real world research. 2nd ed. Blackwell: Oxford.

Rosenau, P. (1992) Post-modernism and the social sciences. New Jersey: Princeton University Press. 
Røtvold, K., \& Wynn, R. (2015). Involuntary psychiatric admission: The referring general practitioners' assessment of patients' dangerousness and need for psychiatric hospital treatment. Nordic Journal of Psychiatry, 69(8), 637-642.

Rowe. J. Jaye, C. (2017) Caring for self-harming patients in general practice. J Prim Health Care. 2017;9(4):279-285. doi:10.171/HC17023

Roy, A. Roy, M. Janal, M. (2010) Suicide attempts and ideation in African-American type 1 diabetic patients. Psychiatry Res; 179:53-6.

Royal College of Psychiatrists (2004). Assessment Following Self-Harm in Adults. Royal College of Psychiatrists: London. Available from: http://www.rcpsych.ac.uk/pdf/Assessment\%20following\%20SH\%20CR122.pdf [Accessed on 03/06/2016]

Royal College of Psychiatrists (2011) Rethinking risk to others in mental health services. Final report of a scoping group. London: Royal College of Psychiatrists. Available from: http://www.rcpsych.ac.uk/pdf/CR150\%20rethinking\%20risk.pdf [accessed on 13/06/2016]

Rush, W.A. Whitebird, R.R. Rush, M.R. (2008) Depression in patients with diabetes: does it impact clinical goals? J Am Board Fam Med; 21:392-7.

Sadowski, L.S. Kee, R.A. VanderWeele, T.J. et al. (2009) Effect of a housing and case management program on emergency department visits and hospitalizations among chronically ill homeless adults: a randomized trial. JAMA; 301:1771-8

Samaritans (2012) Suicide Statistics Report 2012 Data for 2008-2010. Available from:

http://www.samaritans.org/sites/default/files/kcfinder/files/Suicide\%20Statistics\%20 Report\%202012.pdf [accessed on 05/04/2017]

Samaritans (2015) Suicide statistics report 2015. Available from: http://www.samaritans.org/sites/default/files/kcfinder/branches/branch96/files/Suicide_statistics_report_2015.pdf [Accessed on 24/03/2017] 
Samaritans (2016) Suicide statistics report 2016. Available from:

https://www.samaritans.org/sites/default/files/kcfinder/files/Samaritans\%20suicide\% 20statistics\%20report\%202016.pdf. Accessed on 30/09/2018

Sandelowski, M. Docherty, S. Emden, C. (1997) Focus on qualitative methods. Qualitative metasynthesis: issues and techniques. Res Nurs Health; 20:365-71.

Sandelowski M. \& Barroso J. (2003) Classifying the findings in qualitative studies. Qualitative Health Research. 13, 905-923.

SANE (2017) Self-harm The 'secret self'. Available from:

http://www.sane.org.uk/uploads/self-harm.pdf [accessed on 05/04/2017]

Sarantakos, S. (2005). Social Research. (3rd ed.). Melbourne: Macmillan Education.

Sasson, C. Rogers, M.A. Dahl, J. Kellermann, A.L. (2010) Predictors of survival from out-of-hospital cardiac arrest: a systematic review and meta-analysis. Circ Cardiovasc Qual Outcomes 2010;3:63-81.3.

Saunders, K.E.A. Hawton, K. Fortune, S. Farrell, S. (2012) Attitudes and knowledge of clinical staff regarding people who self-harm: A systematic review. Journal of Affective Disorders 139 (2012) 205-216

Saunders, M. Lewis, P. Thornhill, A (2007) Research Methods for Business Students. Prentice Hall: London

Savage v South Essex Partnership NHS Foundation Trust [2008] UKHL 74.

Schindeler, P. Stegink-Jansen, C.W. (2011) Introduction: psychosocial issues at hand. J Hand Ther. 24 (2): 80-81, 2011

Schomerus, G. Angermeyer, M. (2008). Stigma and its impact on help-seeking for mental disorders: what do we know? Epidemiologia e Psichiatria Sociale 17, 31-37

Schwandt, T. A. (2000). Three epistemological stances for qualitative inquiry: Interpretivism, hermeneutics, and social constructionism. In N. K. Denzin \& Y. S. Lincoln (Eds), Handbook of qualitative research (2nd ed., pp. 189-213). Thousand Oaks, CA: Sage. 
Scoliers, G. Portzky, G. Madge, N. Hewitt, A. Hawton, K. De Wilde, J. Ystgaard, E. Arensman, M. Leo, D. Fekete, S. Van Heeringn, K. (2008) Reasons for adolescent deliberate self-harm: a cry of pain and/or a cry for help? Findings from the child and adolescent self-harm in Europe (CASE) study. Soc Psychiatry Psychiatr Epidemiol Epub ahead of print DOI 10.1007/s00127-008-0469-z

Scott R (2000) Evaluation of a mobile crisis program: effectiveness, efficiency, and consumer satisfaction. Psychiatric Services. 51, 9, 1153-1156.

Scott, J. Strickland, A.P. Warner, K. (2014) Describing and predicting frequent callers to an ambulance service: analysis of 1 year call data. Emerg Med J; 31:40814.

Seal v Chief Constable of South Wales Police (2007) UK HL 31

Sessay v South London and Maudsley NHS Foundation Trust (2011) EWHC 2617 (QB)

SG (2017) Scottish Government. Available at: http://www.gov.scot/About/Performance/scotPerforms/partnerstories/NHSScotlandp erformance/ambulanceStandard. 2012 (Accessed 4th January 2017).

Shaban, R. (2006) Paramedics clinical judgment and mental health assessments in emergency contexts: research, practice and tools of the trade. JEHPC; 4.

Shaban, R.Z (2005a.) Paramedic clinical judgment of mental illness: Representations of official accounts. Journal of Emergency Primary Health Care (JEPHC). Vol.3, Issue 4

Shaban, R.Z. (2005 b.) Accounting for assessments of mental illness in paramedic practice: A new theoretical framework Journal of Emergency Primary Health Care. JEPHC; Vol.3, Issue 3

Shaban, R.Z. (2004a) Mental health and mental illness in paramedic practice: A warrant for research and inquiry into accounts of paramedic clinical judgment and decision-making Journal of Emergency Primary Health Care (JEPHC), Vol.2, Issue 3-4. 
Shaban, R.Z. Wyatt-Smith, C.M. Cumming, J. (2004) Uncertainty, error and risk in human clinical judgment: Introductory theoretical frameworks in paramedic practice. Journal of Emergency Primary Health Care 2004, 2(1-2)

Sharp, Richard; Thompson, Sean. (2016) Understanding the Place of Advance Directives in Paramedic Pre-Hospital Care. Whitireia Nursing \& Health Journal; Porirua City Iss. 23: 13-19.

Shaw, L. Ford, G. Exley, C. Flynn, D. McMeekin, P. Rodgers, H. Snooks, H. Tyrell, P. Vale, L. Price, C. (2016) Paramedic acute stroke treatment assessment (pasta) trial. EMJ. Volume 33, Issue 9

Sheridan, E.P. Teplin, L (1982) Police-referred psychiatric emergencies: advantages of community treatment. Journal of Community Psychology 9:140-147.

Shumway, M. Boccellari, A. O’Brien, K. Okin, R.L. (2008) Cost-effectiveness of clinical case management for ED frequent users: results of a randomized trial. Am J Emerg Med. 26:155-64

Sidani, S. Fox, M. (2014) Patient-centered care: clarification of its specific elements to facilitate interprofessional care. J. Interprofessional Care. 28 (2), 134-141. http://dx.doi.org/10.3109/13561820.2013.862519

Sikes, P. (2008) Researching research cultures: The case of new universities. In Researching education from the inside: Investigations from within, eds Sikes, P and Potts, A, 144-58. London: Routledge.

Silverman, M. (2011) Challenges to classifying suicidal ideations, communications, and behaviours. In: Connor, R. Platt, S. Gordon, J. (Eds.), International Handbook of Suicide Prevention Research, Policy and Practice. Wiley-Blackwell, UK, pp. 9-26.

Simpson, P. Thomas, R. Bendall, J. Lord, B. Lord, S. Close, J. (2017) 'Popping nana back into bed' - a qualitative exploration of paramedic decision making when caring for older people who have fallen. BMC Health Services Research (2017) 17:299

Skegg, K. (2005) Self-harm. The Lancet. 366(9495):1471-1483. 
Slade, M. Byford, S. BarratT, B. Lloyd-Evans, B. Gilburt, H. Osborn, D.P.J. Skinner, R. Thornicroft, G. Johnson, S (2010) 'Alternatives to standard acute in-patient care in England: short-term clinical outcomes and cost-effectiveness'. The British Journal of Psychiatry. vol 197, pp s14-s19.

Smyth, A., \& Holian, R. (2008). Credibility Issues in Research from within Organisations. In P. Sikes \& A. Potts (Eds.), Researching education from the inside (pp. 33-47). New York, NY: Taylor \& Francis

Snooks H, Anthony R, Chatters R, Cheung W-Y, Dale J, Donohoe R, Gaze S, Halter M, Koniotou M, Logan P, Lyons R, Mason S, Nicholl J, Phillips C, Phillips J, Russell I, Siriwardena AN, Wani M, Watkins A, Whitfield R \& Wilson L (2012) Support and assessment for fall emergency referrals (SAFER 2) research protocol: cluster randomised trial of the clinical and cost effectiveness of new protocols for emergency ambulance paramedics to assess and refer to appropriate community-based care.. BMJ Open, 2(6).

Snooks HA, Anthony R, Chatters R, Dale J, Fothergill R, Gaze S, Halter M, Humphreys I, Koniotou M, Logan P, Lyons R, Mason S, Nicholl J, Peconi J, Phillips C, Phillips J, Porter A, Siriwardena AN, Smith G, Toghill A, Wani M, Watkins A, Whitfield R, Wilson L, Russell IT (2017). Support and Assessment for Fall Emergency Referrals (SAFER) 2: Cluster randomised trial and systematic review of clinical and cost effectiveness of new protocols for emergency ambulance paramedics to assess older people following a fall with referral to community based care when appropriate. Health Technology Assessment. 21(13), 1-218.

Snooks HA, Anthony R, Chatters R, Dale J, Fothergill RT, Gaze S, Halter M, Humphreys I, Koniotou M, Logan P, Lyons RA, Mason S, Nicholl J, Peconi J, Phillips C, Porter A, Siriwardena AN, Wani M, Watkins A, Wilson L \& Russell IT (2017) Paramedic Assessment of Older Adults After Falls, Including Community Care Referral Pathway: Cluster Randomized Trial. Ann Emerg Med 70(4), 495505.e28. 
Snooks, H. Halter, M. Close, J.C.T. Cheung, W.Y. Moore, F. Roberts, S. E (2006) Emergency care of older people who fall: a missed opportunity. Qual Saf Health Care. 2006 Dec; 15(6): 390-392.

Social Care Institute for Excellence (2006) Using qualitative research in systematic reviews: older people's views of hospital discharge. How knowledge works in social care. SCIE, London.

Sommer-Rotenberg, D. (1998) Suicide and language. CMAJ;159:239-40

Spillane, L.L. Lumb, E.W. Cobaugh, D. J. Wilcox, S.R. Clark, J.S. Schneider, S.M. (1997) Frequent users of the emergency department: can we intervene? Acad Emerg Med. 4:574-80.

Squires, J.P. Mason, S. (2004) Developing alternative ambulance response schemes: analysis of attitudes, barriers, and change. Emergency Medicine Journal. 21, 724727.

Stanley, B. Gameroff, M.J. Michalsen, V. \& Mann, J.J. (2001) Are suicide attempters who self-mutilate a unique population? Am. J. Psychiat. 2001;158:3.427432.

Stiell, I.G. Wells, G.A. Field, III B.J. Spaite, D.W. De Maio, V.J. Ward, R. (1999) Improved Out-of-Hospital Cardiac Arrest Survival Through the Inexpensive Optimization of an Existing Defibrillation Program: OPALS Study Phase II. JAMA: The Journal of the American Medical Association. 281: 1175-1181.

Strauss, A. \& Corbin, J. (1990) Basics of qualitative research: Grounded theory procedures and techniques. Newbury Park, CA: Sage.

Strauss, A. (1993) Continual Permutations of Action, Aldine De Gruyter, New York. Strauss, A. Corbin, J. (1994). "Grounded Theory Methodology." In NK Denzin \& YS Lincoln (Eds.) Handbook of Qualitative Research (pp. 217-285). Thousand Oaks, Sage Publications.

Strauss, A. Corbin, J. (1998). Basics of qualitative research: Techniques and procedures for developing grounded theory. (2nd ed.). Thousand Oaks, CA: Sage. 
Strauss, A. L. (1987). Qualitative analysis for social scientists. New York:

Cambridge University Press.

Streubert, H.J. Carpenter, D.R. (1999) Qualitative research in nursing: advancing the humanistic imperative. 2nd Edition Philadelphia: Lippincott

Stroup, T.C. (2011) Substance use and schizophrenia: adverse correlates in the CATIE study sample. Schizophrenia Research. 132,177-182.

Stuart v Kirkland-Veenstra [2009]

Sun, B.C. Burstin, H.R. Brennan, T.A. (2003) Predictors and outcomes of frequent emergency department users. Acad Emerg Med. 2003; 10:320-8.

Suokas, J. Lonnqvist, J. (1989) Work stress has negative effects on the attitudes of emergency personnel towards patients who attempt suicide. Acta Psychiatr Scand; 79: $474-80$

Suokas, J. Suominen, K. Lonnqvist, J. (2009) The attitudes of emergency staff toward attempted suicide patients: a comparative study before and after establishment of a psychiatric consultation service. Crisis 30, 161-165.

Suserund, B. Blomquist, M. Johansson, I. (2002) Experiences of threats and violence in the Swedish Ambulance Service. Accid Emerg Nurs; 10:127-35.

Squires, J.P. Mason, S. (2004) Developing alternative ambulance response schemes: analysis of attitudes, barriers, and change. Emergency Medicine Journal 21, 724-727

Parsons, T. (1958) Definitions of health and illness in the light of American values. In: Jaco, E.G. (Ed.), Patients, Physicians and Illness: Sourcebook in Behavioral Science and Medicine. The Free Press, Glencoe, pp. 165-187

TAC (2015) Overlooked in the Undercounted: The Role of Mental Illness in Fatal Law Enforcement Encounters. Available from: http://www.treatmentadvocacycenter.org/overlooked-in-the-undercounted [accessed on $01 / 10 / 2018]$ 
Tangherlini, T.R. (2000) Heroes and Lies: Storytelling Tactics among Paramedics. Folklore 111 (2000):43- 66

Taylor, T. Hawton, K. Fortune, S. (2009) Attitudes towards clinical services among people who self-harm; systematic review. Br J Psychiatry; 194:104-10.

Teplin, L (1986) Keeping the Peace: The Parameters of Police Discretion in Relation to the Mentally Disordered. National Institute of Justice Final Report. Washington, DC, US Department of Justice, 1986. Available from: https://www.ncjrs.gov/pdffiles1/jr000244c.pdf [accessed on 12/01/2016]

Teplin, L. A. (2000). Keeping the peace: Police discretion and mentally ill persons. National Institute of Justice Journal. 244, 8-15.

Teplin, L. Pruett, N (1992) Police as street-corner psychiatrist: managing the mentally ill. International Journal of Law and Psychiatry. 15:139-156, 1992,

Thornicroft G (2008). Stigma and discrimination limit access to mental health care. Epidemiologia e Psichiatria Sociale 17, 14-19.

The Commission on Acute Adult Psychiatric Care (2015). Improving acute inpatient psychiatric care for adults in England. Interim report. London: The Commission on Acute Adult Psychiatric Care. Available from: www.caapc.info/ [accessed on 23/06/2015]

The Justice Gap (2015) Tazer use in Mental Health. Available from: http://thejusticegap.com/2015/12/12158/ [Accessed on 22/01/17]

Thornicroft, G. (2011) 'Physical health disparities and mental illness: the scandal of premature mortality', The British Journal of Psychiatry 199(6), 441-2. doi:10.1192/bjp.bp.111.092718

Timmermans, S. (1999a). Sudden death and the myth of CPR. Philadelphia: Temple University Press.

Timmermans, S. (1999b). When death isn't dead: implicit social rationing during resuscitative efforts. Sociological Inquiry. 69, 51-75. 
Townsend, A. Cox, M. Li, L.C. (2010) Qualitative Research Ethics: Enhancing Evidence-Based Practice in Physical Therapy. Physical Therapy. Volume 90 Number 4

Townsend, R. Luck, M. (2009). Protective jurisdiction, patient autonomy and paramedics: the challenges of applying the NSW Mental Health Act. Australasian Journal of Paramedicine. 7(4).

Treatment Advocacy Center (2005) Briefing Paper: Law enforcement and people with severe mental illnesses. Available from:

Center:http://www.popcenter.org/problems/mental_illness/PDFs/TAC_2005a.pdf. [accessed on 01/02/2016]

Tuli, F. (2010) The Basis of Distinction Between Qualitative and Quantitative Research in Fekede Tuli (2010) Social Science: Reflection on Ontological, Epistemological and Methodological Perspectives 1 Ethiop. J. Educ. \& Sc. Vol. 6 No 1 September 2010

Turnbull, G. Chalder, T. (1997) Effects of education on attitudes to deliberate selfharm. Psychiatric Bulletin 21, 334-335

Tzeng, W.C. \& Lipson, J.C (2004) The cultural context of suicide stigma in Taiwan. Qualitative Health Research. 14, 345-358

United Kingdom Parliament (1997) The New NHS: Modern, Dependable, Cm 3807. London: HMSO

UK Government (2017) Terms of reference-Independent review of the Mental Health Act (1983). Available from: https://www.gov.uk/government/publications/mentalhealth-act-independent-review/terms-of-reference-independent-review-of-themental-health-act-1983 [accessed on 10/102017]

United Nations (2006). Convention on rights of people with disabilities and optional protocol. New York: United Nations. Available from:

http://www.un.org/disabilities/documents/convention/convoptprot-e.pdf. [Accessed on $2 / 08 / 2016]$ 
United Nations (2011). Toward universal participation and implementation:

Factsheet \#1. New York: United Nations. Available from:

https://treaties.un.org/doc/source/events/2011/Press_kit/fact_sheet_1_english.pdf. [Accessed on 2/08/2016]

United Nations (2014) General comment no. 1. Article 12: Equal recognition before thelaw. New York: United Nations. Available from: https://documents-ddsny.un.org/doc/ UNDOC/GEN/G14/031/20/PDF/G1403120.pdf. [Accessed on $2 / 08 / 2016]$

Van Heeringen, K. (2002) The international handbook of suicide and attempted suicide. Chichester: Wiley

Vaughn, M.G. Salas-Wright, C.P. DeLisi, M. Larson, M. (2015) Deliberate self-harm and the nexus of violence, victimization, and mental health problems in the United States. Psychiatry Research. Vol 225: Issue 3. p 588-595

Verheij, R. (1996). Explaining urban-rural variations in health: a review of interactions between individual and environment. Social Science \& Medicine, 42, 923-935.

Vickers, B. (2000). Memphis, Tennessee, police department's crisis intervention team. U.S. Available from: https://www.ncjrs.gov/pdffiles1/bja/182501.pdf . [accessed on 12/4/2017]

Vivekananda, K. (2000). Interating models for understanding self-injury. Psychotherapy in Australia. 7:189725.

Wahyuni, D. (2012) The Research Design Maze: understanding paradigms, cases, methods and methodologies. Journal of applied management accounting research. 10(1), pp.69-80.

Walsh B. (2007) Clinical assessment of self-injury: a practical guide. J Clin Psychol. 63(11):1057-1068.

Warm, A. Murray, C. Fox, J. (2002). Who helps? Supporting people who self-harm. Journal of Mental Health. 11: 12197130. 
WAST (2016) Ambulance Services in Wales, November 2016. Available from: http://gov.wales/docs/statistics/2016/161229-ambulance-services-november-2016en.pdf (Accessed 4th January 2017).

Watson, A. C., Morabito, M. S., Draine, J. (2008). Improving police response to persons with mental illness: A multilevel conceptualization of CIT. International Journal of Law and Psychiatry. 31: 359-368.

Webley v St George (2014) Webley v St George's Hospital NHS Trust and anr [2014] EWHC 299

Wenger, E. (1998) Communities of Practice: Learning, Meaning and Identity. Cambridge University Press, Cambridge.

Warm, A. Murray, C. Fox, J. (2002). Who helps? Supporting people who self-harm. Journal of Mental Health. 11: 12197130.

Westling, S. Ahrén, B. Träskman-Bendz, L. Westrin, A. (2004) High CSF-insulin in violent suicide attempters. Psychiatry Res; 129:249-55.

WG (2015) National Assembly for Wales Health and Social Care Committee Alcohol and substance misuse. Available from:

http://www.assembly.wales/laid\%20documents/cr-ld10329/cr-ld10329-e.pdf [accessed on 12/08/2016]

WG (2015) Welsh Government Inquiry into alcohol and substance misuse. College of Paramedics written response. Available from: http://www.senedd.assembly.wales/documents/s36259/ASM\%2003\%20Wales\%20C ollege\%20of\%20Paramedics.pdf [Accessed on 22/11/2016]

WG (2018) Welsh Government Health, Social Care and Sport Committee's inquiry into suicide prevention in Wales: Welsh Ambulance Service oral submission. Available from: http://senedd.assembly.wales/mgIssueHistoryHome. aspx ?IId=20058\&Opt=0 [accessed on 08/09/2018] 
Whitley, E. Gunnell, D. Dorling, D. Davey-Smith, G. (1999). Ecological study of social fragmentation, poverty, and suicide. British Medical Journal, 319: 1034-1037.

WHO (2000) World Health Organization Preventing Suicide: A Resource for General Physicians Mental and Behavioural Disorders, Department of Mental Health. Available from: http://www.who.int/mental_health/media/en/56.pdf. [accessed on 12/03/2015]

Wickremsinhe, M. N. (2018) Emergency involuntary treatment law for people with mental disorders. A comparative analysis of legislation in LMICs. International Journal of Law and Psychiatry. 56 (2018) 1-9

Winkler, T. (2013) Misuse of the Emergency Medical Services system: Frequent flyers and ambulance abusers. Available from:

http://www.academia.edu/4538036/Misuse_of_the_Emergency_Medical_Services_s ystem_Frequent_flyers_and_ambulance_abusers. [Accessed on 09/02/2016]

Woollard, M. Furber, R (2010). The College of Paramedics (British Paramedic Association) position paper regarding the Joint Royal Colleges Ambulance Liaison Committee recommendations on paramedic intubation. Emerg Med J, 27(3): 167170. doi:10.1136/emj.2009.088443

Worster, A. Gilboy, N. Fernandes, C.M. (2004) Assessment of inter-observer reliability of two five-level triage and acuity scaes: a randomized controlled trial. Canadian Journal of Emergency Medicine. 6 (4), 240-245.

Yip, P. Callanan, C. Yuen, H. P. (2000). Urban/rural and gender differentials in suicide rates: East and West. Journal of Affective Disorders. 57, 99-106.

Ystgaard, M. Reinholdt, N. P. Husby, J. Mehlum, L. (2003) Deliberate Self Harm in adolescents. Tidsskrift for den Norske Laegeforening. 123: 2241-2245

Zahl, D. Hawton, K. (2004) Repetition of deliberate self-harm and subsequent suicide risk: long-term follow-up study in 11,583 patients. Br J Psychiatry. 185: 7075. 
Zdanowicz, M. (2001). A sheriff's role in arresting the mental illness crisis. Sheriff, 53(3), 2-4.

Zealberg, J. J. Christie, S. D. Puckett, J. A. (1992). A mobile crisis program collaboration between emergency psychiatric-services and police. Hospital and Community Psychiatry, 43, 612-615.

Zealberg, J. J. Christie, S. D. Puckett, J. A. (1992). A mobile crisis program collaboration between emergency psychiatric-services and police. Hospital and Community Psychiatry. 43: 612-615.

Zimmermann, P.G. (2001) The case for a universal, valid, reliable 5-tier triage acuity scale for U.S. emergency departments. J Emerg Nurse; 27: 246-54.

Zuckerman, S. Shen, Y.C. (2004) Characteristics of occasional and frequent emergency department users: do insurance coverage and access to care matter? Med Care. 42:176-82. 


\section{Appendix A}

\section{NICE (2004 p.29) recommend the following key aims and objectives in the treatment of Self-Harm:}

\section{Respect, understanding and choice}

- People who SH should be treated with the same care, respect and privacy as any patient, and health professionals should take account of likely distress associated with SH.

\section{Staff training}

- Staff who have contact with people who SH should be provided with appropriate training to equip them to understand and care for people who $\mathrm{SH}$.

\section{Activated charcoal}

- Ambulance and ED services should ensure that activated charcoal is immediately available to staff at all times.

\section{Triage}

- All people who SH should be offered a psychosocial assessment at triage. Assessment should determine a person's mental capacity, their willingness to remain for further (psychosocial) assessment, their level of distress and the possible presence of mental illness.

- Consideration should be given to introducing the Australian Mental Health Triage Scale.

- If a person who has SH has to wait for treatment, they should be offered an environment that is safe, supportive and minimises any distress.

\section{Treatment}

- People who SH should be offered treatment for the physical consequences of $\mathrm{SH}$, regardless of their willingness to accept psychosocial assessment or psychiatric treatment.

- Adequate anaesthesia and/or analgesia should be offered to people who have self-injured throughout the process of suturing or other painful treatments.

- Full information should be provided about the treatment options, and all efforts necessary should be made to ensure that someone who has self-harmed can give, and has the opportunity to give, meaningful and informed consent before any and each procedure (for example, taking the person to hospital by ambulance) or treatment is initiated.

\section{Assessment of needs}

- All people who have self-harmed should be offered an assessment of needs, which should be comprehensive and include evaluation of the social, psychological and motivational factors specific to the act of self-harm, current suicidal intent and hopelessness, as well as a full mental health and social needs assessment. 


\section{Assessment of risk}

- All people who have self-harmed should be assessed for risk: this assessment should include identification of the main clinical and demographic features known to be associated with risk of further self-harm and/or suicide, and identification of the key psychological characteristics associated with risk, in particular depression, hopelessness and continuing suicidal intent.

\section{Psychological, psychosocial and pharmacological interventions}

- Following psychosocial assessment for people who have self-harmed, the decision about referral for further treatment and help should be based upon a comprehensive psychiatric, psychological and social assessment, including an assessment of risk, and should not be determined solely on the basis of having self-harmed. 


\section{NICE (2004 p.48) key priorities for implementation in caring for SH}

- Rapid assessment of physical and psychological need (triage)

- Effective engagement of service user (and carers where appropriate)

- Effective measures to minimise pain and discomfort

- Timely initiation of treatment, irrespective of the cause of self-harm

- Harm reduction (from injury and treatment; short-term and longer-term)

- Rapid and supportive psychosocial assessment (including risk assessment and comorbidity)

- Prompt referral for further psychological, social and psychiatric assessment and treatment when necessary

- Prompt and effective psychological and psychiatric treatment when necessary

- An integrated and planned approach to the problems of people who self-harm, involving primary and secondary care, mental and physical healthcare personnel and services, and appropriate voluntary organisations

- Ensuring that the special issues applying to children and young people who have self-harmed are properly addressed, such as child protection issues, confidentiality, consent and competence. 


\section{Appendix C}

\section{The recommendations from NICE (2004 p.55) dedicated to ambulance staff}

- When attending a person who has self-harmed, ambulance staff should urgently establish the likely physical risk, and the person's emotional and mental state, in an atmosphere of respect and understanding

- Ambulance staff should be trained in the assessment and early management of SH. Training should address the different methods of SH and the appropriate treatments, the likely effects if untreated, and issues of consent and mental capacity, as these apply both to adults, and to children and young people.

- If following an act of self-injury, the service user does not require emergency treatment in the ED, ambulance staff should consider, having taken full account of the service user's preferences, taking the service user to an alternative appropriate service, such as a specialist mental health service. The decision to do so should be taken jointly between the ambulance staff, the service user and the receiving service.

- Ambulance Trusts, the ED and Mental Health Trusts should work in partnership to develop locally agreed protocols for ambulance staff to consider alternative care pathways to emergency departments for people who have self-harmed, where this is appropriate and does not increase the risks to the service user.

- In cases of self-poisoning, ambulance staff should obtain all substances and/or medications found at the scene of an emergency call, whether thought to be involved in the overdose or not, and pass these to staff upon arrival at the emergency department.

- Unless the service user's clinical condition requires urgent treatment that should not be delayed, ambulance staff should record relevant information about the service user's home environment, social and family support network, and history leading to self-harm, as well as the service user's initial emotional state and level of distress. This information should be passed to ED staff.

- When transporting people who have self-harmed to an ED, wherever possible, ambulance staff should take into account the service user's preferences when more than one emergency department facility exists within a reasonable distance, unless doing so significantly increases the risk to the service user, or when one department has specialised in the treatment of people who have self-harmed.

- When a person who has self-poisoned presents to the ambulance service within 1 hour of ingestion and is fully conscious and able to protect his or her own airway, ambulance staff should consider offering activated charcoal at the earliest opportunity. Activated charcoal should be offered only when the substance(s) ingested are likely to be adsorbed by activated charcoal and when the person is considered to be at risk of significant harm.

- Activated charcoal may also be considered between 1 and 2 hours after ingestion as there is some evidence that activated charcoal may still be effective in reducing absorption, especially if the ingested substance delays gastric emptying, such as tricyclic antidepressants. Activated charcoal should be offered only when the substance(s) ingested are likely to be adsorbed by activated charcoal and when the person is considered to be at risk of significant harm. 
- In the emergency treatment of opioid overdose when using intravenous naloxone, ambulance staff should adhere to the guidelines established by the Joint Royal Colleges Ambulance Liaison Committee. Particular attention should be given to the possible need for repeated doses of naloxone and frequent monitoring of vital signs, because the effects of naloxone are short lived in comparison with the effects of most opioids and service users frequently relapse once the effect of naloxone has worn off. All people who have overdosed with opioids should be conveyed to hospital, even if the initial response to naloxone has been good.

- The ambulance services should ensure that there is rapid access to TOXBASE and the NPIS so that their crew can gain additional information on substances and/or drugs ingested in cases of self-poisoning in order to assist in decisions regarding urgent treatment and the transfer of patients to the most appropriate facilities.

- When people who have self-harmed are considering refusing further treatment, ambulance staff should assess mental capacity and provide information about the potential consequences of not receiving treatment when attempting to gain valid consent. When consent is withheld, the guidance on consent and capacity in this guideline should be followed.

- PCTs, in conjunction with acute and mental health trusts, should consider the level of support needed for the delivery of an adequate pre-hospital care system for self-harm. Specific consideration should be given to the provision of telephone advice to ambulance staff from crisis resolution teams, approved social workers and Section 12 approved doctors, regarding the assessment of mental capacity and the possible use of the Mental Health Act in the urgent assessment of people who have self-harmed.

- Ambulance Trusts should regularly update ambulance staff about any change in local arrangements for services available for the emergency treatment of people who have self-harmed.

- Ambulance Trusts should routinely audit incidents of overdose, both to ensure that interventions are being used consistently and effectively, and to monitor adverse incidents. 
Appendix D

\section{Royal College of Psychiatrists RCPsych (2006) guidelines in relation to ambulance staff care for people who SH}

- Ambulance services should work with other organisations to develop care pathways for patients already known to the service, including service users being taken directly to mental health units, primary care, crisis intervention teams or to social services.

- Ambulance trusts, the ED and mental health trusts should work in partnership to develop locally agreed protocols for ambulance staff to consider alternative care pathways to emergency departments for people who have self-harmed, where this is appropriate and does not increase the risks to the service user.

- If the service user does not require treatment in the emergency department, ambulance staff should consider taking the service user to an alternative appropriate service, such as a specialist mental health service.

- Ambulance staff should have access to telephone advice from crisis resolution teams.

- Ambulance staff should have access to telephone advice from approved doctors, regarding the assessment of mental capacity and the possible use of the Mental Health Act in the urgent assessment of people who have self-harmed.

- Ambulance staff should have access to telephone advice from approved social workers. (RCPsych 2006 p.25, p40) 


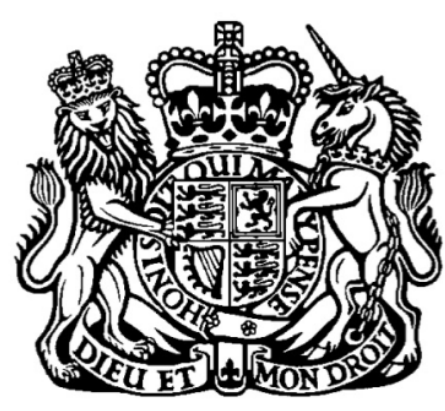

\section{Policing and Crime Act 2017}

CHAPTER 3

\section{THE 'NEW' SECTION 136}

What follows is the full, amended text of section 136 Mental Health Act 1983, as it will be following a commencement order for the Policing and Crime Act 2017 which will bring it in to effect. It also includes the new sections 136A, 136B and 136C. See related posts for the 'new' section 135 and section 138 MHA.

NB! - this is NOT the current law as of 03rd March 2017 - this is the law as it will become, following a commencement order yet to be laid before Parliament. The full Mental Health Act 1983, as it stands today and which will be kept updated once the commencement order takes effect, can be found on the UK Government legislation website.

Section 136 - removal of mentally disordered persons without warrant.

(1) If a person appears to a constable to be suffering from mental disorder and to be in immediate need of care or control, the constable may, if he thinks it necessary to do so in the interests of that person or for the protection of other persons-

(a) remove the person to a place of safety within the meaning of section 135 , or 
(b) if the person is already at a place of safety within the meaning of that section, keep the person at that place or remove the person to another place of safety.

(1A) The power of a constable under subsection (1) may be exercised where the mentally disordered person is at any place, other than-

(a) any house, flat or room where that person, or any other person, is living, or

(b) any yard, garden, garage or outhouse that is used in connection with the house, flat or room, other than one that is also used in connection with one or more other houses, flats or rooms.

(1B) For the purpose of exercising the power under subsection (1), a constable may enter any place where the power may be exercised, if need be by force.

(1C) Before deciding to remove a person to, or to keep a person at, a place of safety under subsection (1), the constable must, if it is practicable to do so, consult-

(a) a registered medical practitioner,

(b) a registered nurse,

(c) an approved mental health professional, or

(d) a person of a description specified in regulations made by the Secretary of State.

(2) A person removed to or kept at a place of safety under this section may be detained there for a period not exceeding the permitted period of detention for the purpose of enabling him to be examined by a registered medical practitioner and to be interviewed by an approved mental health professional and of making any necessary arrangements for his treatment or care.

(2A) In subsection (2), "the permitted period of detention" means-

(a) the period of 24 hours beginning with-

(i) in a case where the person is removed to a place of safety, the time when the person arrives at that place;

(ii) in a case where the person is kept at a place of safety, the time when the constable decides to keep the person at that place; or

(b) where an authorisation is given in relation to the person under section 136B, that period of 24 hours and such further period as is specified in the authorisation.

(3) A constable, an approved mental health professional or a person authorised by either of them for the purposes of this subsection may, before the end of the permitted 
period of detention mentioned in subsection (2) above, take a person detained in a place of safety under that subsection to one or more other places of safety.

(4) A person taken to a place of a safety under subsection (3) above may be detained there for a purpose mentioned in subsection (2) above for a period ending no later than the end of the permitted period of detention mentioned in that subsection.

(5) This section is subject to section 136A which makes provision about the removal and taking of persons to a police station, and the keeping of persons at a police station, under this section.

\section{Section 136A - use of police stations as places of safety.}

(1) A child may not, in the exercise of a power to which this section applies, be removed to, kept at or taken to a place of safety that is a police station.

(2) The Secretary of State may by regulations-

(a) provide that an adult may be removed to, kept at or taken to a place of safety that is a police station, in the exercise of a power to which this section applies, only in circumstances specified in the regulations;

(b) make provision about how adults removed to, kept at or taken to a police station, in the exercise of a power to which this section applies, are to be treated while at the police station, including provision for review of their detention.

(3) Regulations under this section-

(a) may make different provision for different cases;

(b) may make provision that applies subject to specified exceptions;

(c) may include incidental, supplementary or consequential provision or transitional, transitory or saving provision.

(4) The powers to which this section applies are-

(a) the power to remove a person to a place of safety under a warrant issued under section $135(1)$;

(b) the power to take a person to a place of safety under section $135(3 \mathrm{~A})$;

(c) the power to remove a person to, or to keep a person at, a place of safety under section 136(1);

(d) the power to take a person to a place of safety under section 136(3).

(5) In this section- 
(a) "child" means a person aged under 18;

(b) "adult" means a person aged 18 or over.

\section{Section 136B - extension of detention}

(1) The registered medical practitioner who is responsible for the examination of a person detained under section 135 or 136 may, at any time before the expiry of the period of 24 hours mentioned in section 135(3ZA) or (as the case may be) 136(2A), authorise the detention of the person for a further period not exceeding 12 hours (beginning immediately at the end of the period of 24 hours).

(2) An authorisation under subsection (1) may be given only if the registered medical practitioner considers that the extension is necessary because the condition of the person detained is such that it would not be practicable for the assessment of the person for the purpose of section 135 or (as the case may be) section 136 to be carried out before the end of the period of 24 hours (or, if the assessment began within that period, for it to be completed before the end).

(3) If the person is detained at a police station, and the assessment would be carried out or completed at the station, the registered medical practitioner may give an authorisation under subsection (1) only if an officer of the rank of superintendent or above approves it.

\section{Section 136C - protective searches}

(1) Where a warrant is issued under section 135(1) or (2), a constable may search the person to whom the warrant relates if the constable has reasonable grounds for believing that the person-

(a) may present a danger to himself or herself or to others, and

(b) is concealing on his or her person an item that could be used to cause physical injury to himself or herself or to others.

(2) The power to search conferred by subsection (1) may be exercised-

(a) in a case where a warrant is issued under section 135(1), at any time during the period beginning with the time when a constable enters the premises specified in the warrant and ending when the person ceases to be detained under section 135;

(b) in a case where a warrant is issued under section 135(2), at any time while the person is being removed under the authority of the warrant.

(3) Where a person is detained under section 136(2) or (4), a constable may search the person, at any time while the person is so detained, if the constable has reasonable grounds for believing that the person- 
(a) may present a danger to himself or herself or to others, and

(b)is concealing on his or her person an item that could be used to cause physical injury to himself or herself or to others.

(4) The power to search conferred by subsection (1) or (3) is only a power to search to the extent that is reasonably required for the purpose of discovering the item that the constable believes the person to be concealing.

(5) The power to search conferred by subsection (1) or (3) -

(a) does not authorise a constable to require a person to remove any of his or her clothing other than an outer coat, jacket or gloves, but

(b) does authorise a search of a person's mouth.

(6) A constable searching a person in the exercise of the power to search conferred by subsection (1) or (3) may seize and retain anything found, if he or she has reasonable grounds for believing that the person searched might use it to cause physical injury to himself or herself or to others.

(7) The power to search a person conferred by subsection (1) or (3) does not affect any other power to search the person.

The full Mental Health Act 1983, which will be updated once the commencement order takes effect, can be found here. 


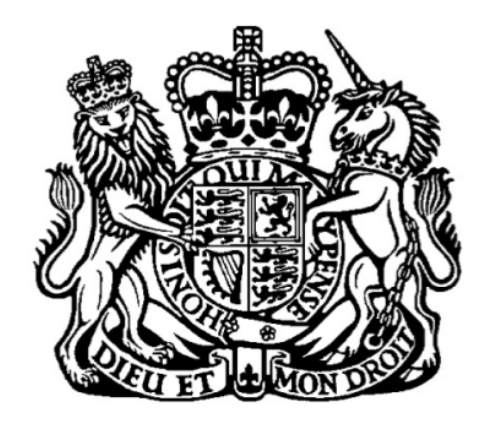

\section{Policing and Crime Act 2017}

CHAPTER 3

\section{THE 'NEW' SECTION 135}

What follows is the full, amended text of section 135 Mental Health Act 1983, as it will be following a commencement order for the Policing and Crime Act 2017 which will bring it in to effect. See related posts for the 'new' section $136 \mathrm{MHA}$, including sections 136A, 136B and 136C, as well as section 138.

NB! - this is NOT the current law as of 03rd March 2017 - this is the law as it will become, following a commencement order yet to be laid before Parliament. The full Mental Health Act 1983, as it stands today and which will be kept updated once the commencement order takes effect, can be found on the UK Government legislation website.

\section{Section 135 - Warrant to search for and remove patients.}

(1) If it appears to a justice of the peace, on information on oath laid by an approved mental health professional, that there is reasonable cause to suspect that a person believed to be suffering from mental disorder-

(a) has been, or is being, ill-treated, neglected or kept otherwise than under proper control, in any place within the jurisdiction of the justice, or

(b) being unable to care for himself, is living alone in any such place,the justice may issue a warrant authorising any constable to enter, if need be by force, any premises 
specified in the warrant in which that person is believed to be, and, if thought fit, to remove him to a place of safety with a view to the making of an application in respect of him under Part II of this Act, or of other arrangements for his treatment or care.

(1A) If the premises specified in the warrant are a place of safety, the constable executing the warrant may, instead of removing the person to another place of safety, keep the person at those premises for the purpose mentioned in subsection (1).

(2) If it appears to a justice of the peace, on information on oath laid by any constable or other person who is authorised by or under this Act or under article 8 of the Mental Health (Care and Treatment)(Scotland) Act 2003 (Consequential Provisions) Order 2005 to take a patient to any place, or to take into custody or retake a patient who is liable under this Act or under the said article to be so taken or retaken-

(a) that there is reasonable cause to believe that the patient is to be found on premises within the jurisdiction of the justice; and

(b) that admission to the premises has been refused or that a refusal of such admission is apprehended, the justice may issue a warrant authorising any constable to enter the premises, if need be by force, and remove the patient.

(3)A patient who is removed to a place of safety in the execution of a warrant issued under subsection (1) or kept at the premises specified in the warrant under subsection (1A), may be detained there for a period not exceeding the permitted period of detention.

(3ZA) In subsection (3), "the permitted period of detention" means-

(a) the period of 24 hours beginning with-

(i) in a case where the person is removed to a place of safety, the time when the person arrives at that place;

(ii) in a case where the person is kept at the premises specified in the warrant, the time when the constable first entered the premises to execute the warrant; or

(b) where an authorisation is given in relation to the person under section $136 \mathrm{~B}$, that period of 24 hours and such further period as is specified in the authorisation.

(3A) A constable, an approved mental health professional or a person authorised by either of them for the purposes of this subsection may, before the end of the permitted period of detention mentioned in subsection (3) above, take a person detained in a place of safety under that subsection to one or more other places of safety.

(3B) A person taken to a place of safety under subsection (3A) above may be detained there for a period ending no later than the end of the permitted period of detention mentioned in subsection (3) above. 
(4) In the execution of a warrant issued under subsection (1) above, a constable shall be accompanied by an approved mental health professional and by a registered medical practitioner, and in the execution of a warrant issued under subsection (2) above a constable may be accompanied-

(a) by a registered medical practitioner;

(b) by any person authorised by or under this Act or under article 8 of the Mental Health (Care and Treatment)(Scotland) Act 2003 (Consequential Provisions) Order 2005 to take or retake the patient.

(5) It shall not be necessary in any information or warrant under subsection (1) above to name the patient concerned.

(6) In this section "place of safety" means residential accommodation provided by a local social services authority under Part 1 of the Care Act 2014 or Part 4 of the Social Services and Well-being (Wales) Act 2014 a hospital as defined by this Act, a police station, an independent hospital or care home for mentally disordered persons or any other suitable place.

(7) For the purpose of subsection (6) -

(a) a house, flat or room where a person is living may not be regarded as a suitable place unless-

(i) if the person believed to be suffering from a mental disorder is the sole occupier of the place, that person agrees to the use of the place as a place of safety;

(ii) if the person believed to be suffering from a mental disorder is an occupier of the place but not the sole occupier, both that person and one of the other occupiers agree to the use of the place as a place of safety;

(iii) if the person believed to be suffering from a mental disorder is not an occupier of the place, both that person and the occupier (or, if more than one, one of the occupiers) agree to the use of the place as a place of safety;

(b) a place other than one mentioned in paragraph (a) may not be regarded as a suitable place unless a person who appears to the constable exercising powers under this section to be responsible for the management of the place agrees to its use as a place of safety.

(8) This section is subject to section 136A which makes provision about the removal and taking of persons to a police station under this section. 


\section{Appendix F: Biosketch}

The paramedic researcher in this study (NR) had been a paramedic for twenty-five years on starting the study. He had held various positions supporting education and clinical practice. He had mentored many student paramedics, taught small groups of paramedics, lectured at higher education and spoken at many national and international conferences. In his clinical practice he had worked in many environments providing basic ambulance care and advanced care in the ED as an Advanced Emergency Practitioner. The researcher had therefore cared for many people who $\mathrm{SH}$, witnessed death by suicide because of $\mathrm{SH}$ and observed the care of other paramedics throughout this time.

Throughout the duration of this study, the researcher paramedic had changed jobs several times, leaving full time clinical practice in the ambulance service to work in an ED, then returning to the ambulance service and eventually ending up in a leadership post. 


\section{Appendix G}

Participant information sheet, Demographics Consent form, and Interview Guide

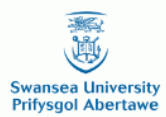

For office use only: Participant number

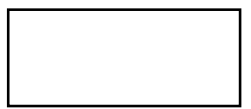

\section{PhD Study: Paramedics' Perceptions of Caring for Those who Self Harm}

\section{Consent Form}

Centre: College of Medicine, Swansea University, Singleton Park, Swansea SA2 8PP

Funder: Welsh Ambulance Service NHS Trust

Full title: "Paramedics Perceptions of Care for Those Who Self Harm"

Principal Investigator (PI): Nigel Rees

PhD supervisors :

Snooks

Professor Frances Rapport

Paramedic Researcher contact details for any queries:

Tel: email:

Please initial all of the statements in the boxes below to signify your consent.

1. I confirm that I have read and understood the information sheet for the above study and have had the opportunity to ask questions.

2. I agree to take part in the above study as a participant in an interview with possible follow up interviews.

3. I understand that my participation is voluntary and that I am free to withdraw at any time, without giving any reason.

4. I understand that the information gathered during the course of the interview(s) will be anonymous.

5. I understand that the interview(s) will be tape recorded, the paramedic researcher will be taking notes, and verbatim quotations from recordings may be used anonymously in the study.

6. I understand that information gathered will be read and analysed by the paramedic researcher and a selected group of academic and research professionals. I give permission for these professionals to have access to this information.

7. I agree to information being used for research purposes and publications (in whole or in part) in peer-reviewed academic journals.

8. I agree to information being used for teaching purposes and publications (in whole or in part) in appropriate educational forms.

Your name:

Date:

Address:

Telephone No(s):

Email:

Signature:

The completed consent form will be kept in a locked cabinet at Swansea University in a study file (1 original) with 1 copy for you. 


\section{PhD Study: Paramedics' Perceptions of Caring for Those who Self Harm (Demographic data)}

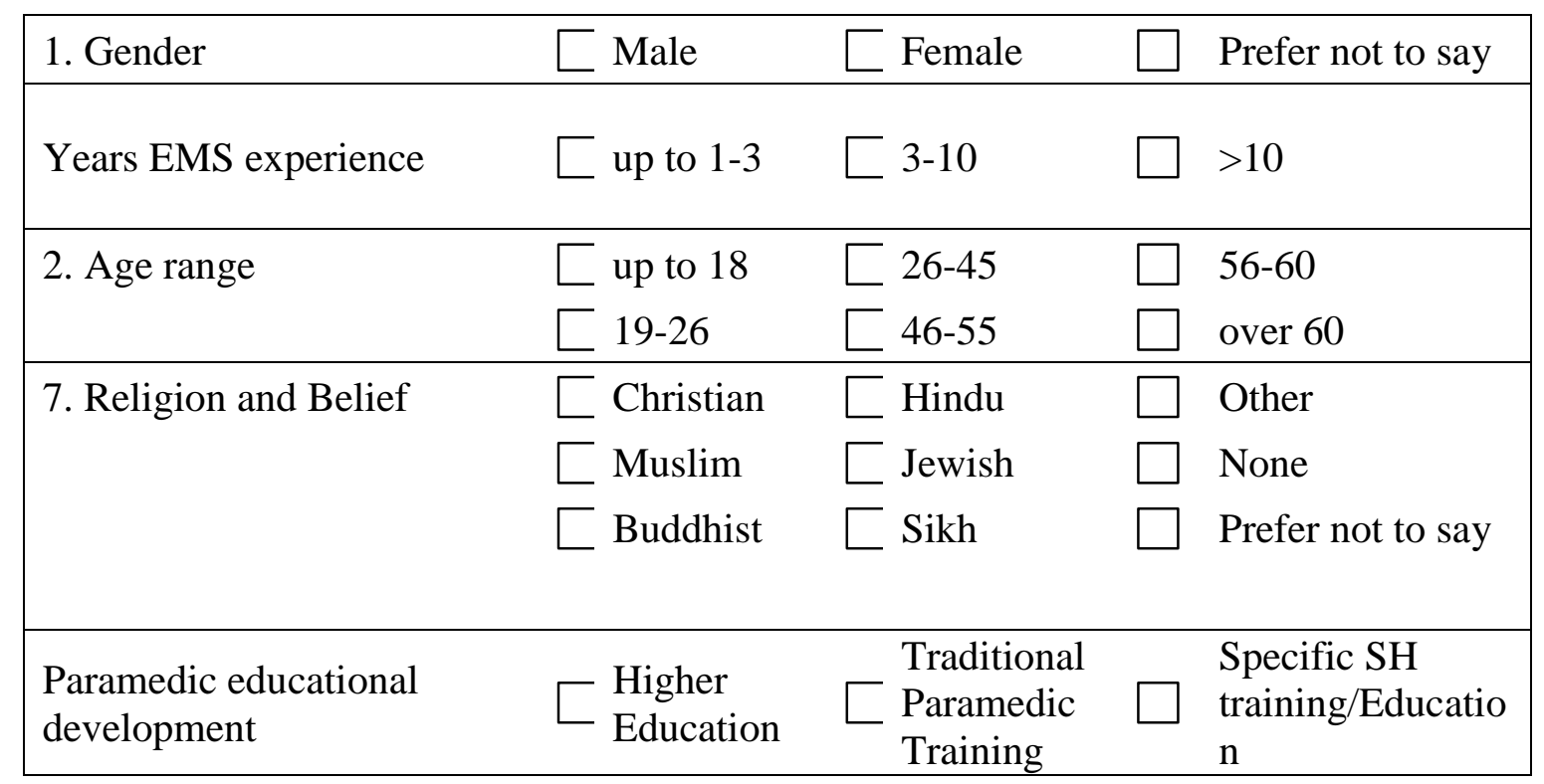




\section{Email:}

\section{"Paramedics' Perceptions of Caring for Those who Self Harm" Information Sheet}

\section{Study:}

The U.K. has one of the highest rates of Self Harm (SH) in Europe at 400 per 100,000 of population, and it is one of the five top causes of acute hospital admissions, increasing by ten per cent every three years. Paramedics are often the first professionals encountered by those who engage in $\mathrm{SH}$, yet few have sought to investigate their care. This study seeks to address this gap, by conducting a deep explorative enquiry to reveal paramedics' perceptions of care for those who SH. Categorys that emerge will be integrated into an educational programme for paramedics, and theory on paramedic care for those who $\mathrm{SH}$ will then be developed.

A paramedic researcher at the College of Medicine, Swansea University and the Welsh Ambulance Services NHS Trust (WAST), would like to invite you to take part in a study being part-funded by WAST that will investigate paramedics' perceptions of care for those who SH.

I have been funded to work with paramedics working in the emergency setting who have experiences of delivering care to people who SH. I wish to understand how paramedics perceive care for those who $\mathrm{SH}$, what, their experiences are of caring for those who $\mathrm{SH}$, the issues and challenges in the pre hospital setting of caring for people who $\mathrm{SH}$, and how this care may be improved.

I would like to invite you to participate in a one-hour interview with the potential of additional follow-up interviews or correspondence to address these issues. The interview will take place in a mutually agreed venue to be decided nearer the time on a day that is mutually convenient for you. At the end of the study you will be provided with brief feedback, which will also be sent to WAST.

The study will build on previous published research about care for those who SH. Members of the research team (paramedic researcher and Supervisory group) have experience of facilitating these kinds of interviews with professionals across Wales.

\section{Reasons for involvement}

Paramedics are often the first point of contact for those who SH, yet little research has taken place that examines the paramedic's perspective of care for Self Harm, nor their potential contribution. Most of the research has been based on "in hospital" studies; there are subsequently gaps in our understandings of this "pre hospital" phase of care. We aim 
to produce research papers and support the development of a tailored educational intervention for paramedics caring for those who $\mathrm{SH}$.

Please read the following information carefully before you decide if you would like to take part and get back to the paramedic researcher if you would like any further information.

\section{What is the purpose of the study?}

I intend to gather the views of paramedics who care for those who SH. I want to learn more about the experiences and challenges that paramedics face when dealing with SH. I want to fully involve paramedics in assessing their care for people who $\mathrm{SH}$, benefits that come as a result and where there might be room for improvement to the care provided.

\section{What would be your role?}

I would like to involve you by asking you to spend an hour (with a break before and after with refreshments) discussing paramedic care for those who $\mathrm{SH}$, your thoughts and experiences of this care, and the particular challenges you face. You will be attending individually. I will listen to your personal experiences, and I will be asking you questions to explore both positive and challenging aspects of this care.

I will use the information we gathered to write a $\mathrm{PhD}$ thesis, academic journal articles for wide audiences and I may also use the information for educational events, teaching events and for presentations.

\section{Practicalities}

The study will be led from Swansea University and your involvement will be for a one hour interview that will take place in a mutually agreed venue which will be an optional, convenient and private location (venue and date will be confirmed by the researcher). You may be requested to take part in further follow-up meetings. If you wish to participate, you should keep this information sheet, sign the consent form you are given, and return it in the stamped-addressed envelope provided as a sign of willingness to participate.

If you decide to take part you are still free to withdraw at any time and without giving any reason. At the beginning of the study, the researcher will contact you with details of date, venue etc for the interview.

\section{Possible benefits of taking part}

Your role in this study will help us to understand paramedics' perceptions of care for those who SH, which may inform policy, practice and assist in the development of a tailored educational intervention for paramedics. It will also be a good opportunity for Continuous Professional Development to shape future paramedic practice.

\section{Confidentiality and anonymity}

The interviews will be tape recorded and notes will be taken. Data will be anonymised and kept securely in locked cabinets at Swansea University. Any data which is transcribed will be anonymized and documents will remain confidential. The information will only be analysed by members of the research team and your name and contact details will not be linked to that material. With your permission, anonymized documents may be used for publication, research, and teaching purposes, and we may include verbatim quotations. Such quotations will be used to describe paramedics' experiences and will not be linked to any individual participant. The study will be conducted in accordance with ethical principles and Swansea University's Code of Ethics and Research Governance.

\section{Funding and ethics}


This study is part funded by the Welsh Ambulance Services NHS Trust. Research and Development Approval has formally been given by the WAST. Research ethics approval was not required as paramedics are participants are by virtue of their NHS employment, in line with UK Health Departments Governance Arrangements for Research Ethics Committees.

\section{Reimbursement for your time or travel}

You will receive full payment for your time and travel expenses in line with Welsh Ambulance Services NHS Trust normal arrangements. (Approved through WAST Research support and permissions). Claim time and travel as per usual practice; your Clinical Team Leader will be required to complete the attached recharge form.

\section{Concerns that this study raises}

Should you have any concerns about taking part in this study or if any anxieties are raised as a result of participating in the study, please contact Nigel Rees

you can contact the anonymous counselling service Network of Staff Support (NOSS),
and for internal WAST concerns over the research please contact Richard Whitfield
Research \& Development Manager on

\section{Further information}

For clarification of any of these details, please contact the researcher on this project:

Mr Nigel Rees Email:

Principal Investigator: Nigel Rees paramedic researcher

Supervisors: Professor Helen Snooks

Professor Frances Rapport

\section{I would like to thank you for your time in reading this.}




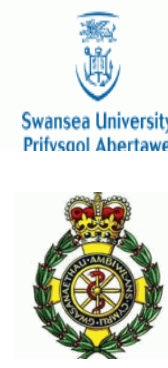

Pre-hospital Emergency Research Unit

Lansdowne Hospital

Sanatorium Road

Cardiff

CF11 8UL

Tel:

Email:

\section{"Paramedics' Perceptions of Caring for Those who Self Harm" Information Sheet}

Dear,

Thank you for agreeing to participate in the above study. We are nearing the stage of data collection and interview. Would you please read the information sheet, sign and complete the enclosed documents, then return them in the envelope supplied.

When I receive the documents I will be contacting you to arrange the interview.

Once again, thank you for agreeing to participate in the study.

Warm Regards

Nigel Rees

MSc, BSc hons, MCPARA

Paramedic Researcher 


\begin{tabular}{|c|c|}
\hline & Interview Guide \\
\hline Pre Amble & $\begin{array}{l}\text { My name is Nigel Rees I want to thank you for taking the } \\
\text { time to meet with me today. I would also like to thank you for } \\
\text { reading and completing the forms sent to you. We are here to } \\
\text { discuss paramedics' perceptions of care for those who Self } \\
\text { Harm. Before starting the interview I would like you to } \\
\text { consider the following definition of Self Harm. Please } \\
\text { remember, this is just one definition of Self Harm: } \\
\text { "An intentional act of self-poisoning or self-injury } \\
\text { irrespective of the type of motivation or degree of suicidal } \\
\text { intent. Thus it includes suicide attempts as well as acts where } \\
\text { little or no suicidal intent is involved (e.g. where people harm } \\
\text { themselves to reduce internal tension, distract themselves } \\
\text { from intolerable situations, as a form of interpersonal } \\
\text { communication of distress or other difficult feelings, or to } \\
\text { punish themselves." (RCPsych2010) }\end{array}$ \\
\hline Questions: & $\begin{array}{l}\text { 1. I have given you this definition so that when we discuss } \\
\text { Self Harm we have a shared understanding. Would you } \\
\text { agree with this definition? } \\
\text { 2. What do you think about the care that you as a paramedic } \\
\text { provide for people who Self Harm? } \\
\text { 3. What do you think about the care that paramedics as a } \\
\text { professional group give to people who Self Harm? } \\
\text { 4. Can you give an example of an incident where a } \\
\text { paramedic provided what you would perceive as a high } \\
\text { standard of care to a person who had Self Harmed? } \\
\text { 5. Can you give an example of an incident where a } \\
\text { paramedic provided what you would perceive as a low } \\
\text { standard to care to a person who had Self Harmed? } \\
\text { 6. Is the care that paramedics give to people who Self Harm } \\
\text { appropriate? } \\
\text { 7he care that those who Self Harm get in an emergency } \\
\text { may affect their future view of care, case management or } \\
\text { future Self Harm incidents. Do you feel paramedic care } \\
\text { should be influence by these factors? } \\
\text { 8. Do you feel your care is sufficiently informed and } \\
\text { supported to deliver care for people who Self Harm? }\end{array}$ \\
\hline $\begin{array}{l}\text { Closing Key } \\
\text { Components: } \\
\text { - Additional } \\
\text { comments } \\
\text { - Next steps } \\
\text { - Thank you }\end{array}$ & $\begin{array}{l}\text { Wrap up notes to self: } \\
\text { 1. Do you have anything else to add? } \\
\text { 2. Is there anything I should have asked? } \\
\text { 3. How did the interview feel for you? } \\
\text { 4. You have agreed to follow up interviews is this still } \\
\text { OK with you? } \\
\text { 5. Thank you for your time. }\end{array}$ \\
\hline
\end{tabular}




\section{NHS R\&D Approvals}

\section{Appendix H:}

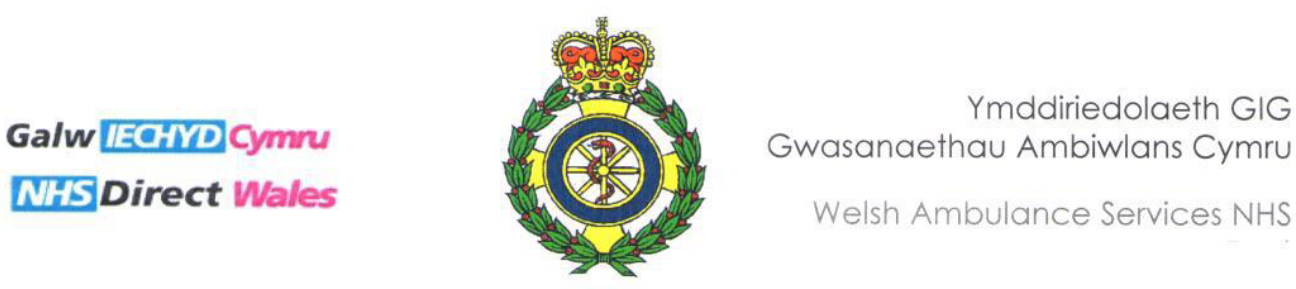

Finance Building, Lansdowne Site Sanatorium Road, Cardiff CF11 8PL

Ffon/Tel 02920932903 Ffacs/Fax 02920237930

www.ambulance.wales.nhs.uk

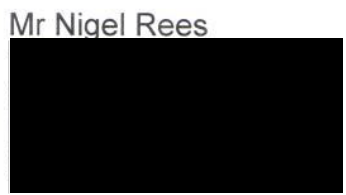

$17^{\text {th }}$ January 2013

Dear Nigel

Paramedics' perceptions of caring for those who Self Harm: An evolved grounded theory exploring the development and impact of a custom educational programme

Your above research proposal has been considered by the WAST R\&D Forum's Research Review Risk Group. I am pleased to inform you that your proposal has been granted NHS R\&D Permission to commence in the Welsh Ambulance Services NHS Trust.

I have spoken to Professor Helen Snooks of Swansea University who informs me that the work you are doing for your doctorate will inform the larger SAFER 4 Project (development of a mental health model-of-care for application and testing in pre-hospital emergency services) which will no doubt be a portfolio study if it is successful in its funding application. I am therefore able to offer you funding support from the Trust as we can class the work that you are doing for your doctorate as a 'Pathway to Portfolio Study'. We will be able to provide funding for any expenses that may be incurred such as overtime payments for staff for interviews and staff travelling expenses but unfortunately we will not be able to pay your doctorate fees.

I look forward to liaising with you during the course of your study and if you have any queries please do not hesitate to contact me.

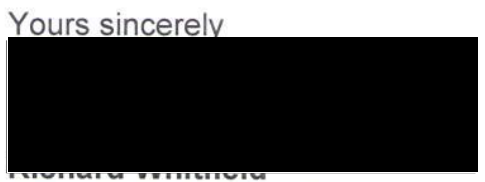

Practice Research \& Development Manager

cc: Paul Hughes, Medical Director

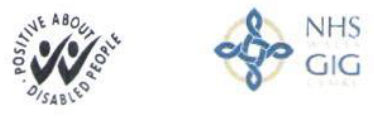

Pencadlys yr Ymddiriedolaeth, Ysbyty HM Stanley, Llanelwy, Sir Ddinbych, LL17 ORS Trust Headquarters, HM Stanley Hospital, St Asaph, Denbighshire, LL17 ORS 
Finance Building, Lansdowne Site

Sanatorium Road, Cardiff CF11 8PL

Ffon/Tel 02920932903 Ffacs/Fax 02920237930

www.ambulance.wales.nhs.uk

18 March 2013

Nigel Rees

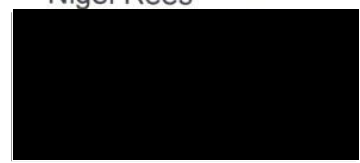

Dear Nigel

Paramedics' perceptions of caring for those who Self Harm: An evolved grounded theory exploring the development and impact of a custom educational program RD037

We have reviewed your research proposal and note that your research project will be interviewing healthcare staff (paramedics) by virtue of their professional role and that there will be no actual patient contact or any individual or identifiable patient data used.

The WAST R\&D Office therefore considers that there are no material ethical issues and that you will not require any formal research ethical approval to carry out your study.

Yours sincerely

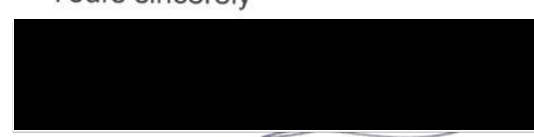

Richard Whitfield

Practice Research \& Development Manager 


\title{
Appendix I
}

\section{Rees, N. Rapport, F. Thomas, G. John, A. Snooks, H. (2014) Perceptions of Paramedic and Emergency Care Workers of those who Self Harm: A Systematic Review of the Quantitative Literature. Journal of Psychosomatic Research. 77: 449-456}

\begin{abstract}
Objective: The U.K. has one of the highest rates of self harm in Europe at 400 per 100,000 of population. Paramedics and emergency staff may be the first professionals encountered, therefore understanding their views and approaches to care is crucial. The aim of this study was to systematically review published quantitative literature relating to paramedic and emergency workers' perceptions and experiences of caring for people who self harm.
\end{abstract}

Methods: CINAHL®, MEDLINE®, OVID $®$ and Psych INFO® databases were searched, PRISMA guidelines were followed, two researchers independently screened titles, abstracts and full papers against a priori eligibility criteria. Data synthesis was achieved by extracting and descriptively analysing study characteristics and findings. Results: 16 studies met inclusion criteria; one included ambulance staff, all used questionnaires. Training, policies and guidelines improved staff knowledge and confidence in caring for people who self harm. Limited access to training was reported, ranging from $75 \%$ to $90 \%$ of staff lacking any. Limited departmental guidelines were also reported. Staff in acute settings exhibited increased feelings of negativity, becoming less positive closer to front line care. Recent studies report positive attitudes amongst emergency staff.

Discussion: Despite guidelines indicating need for education and policies to guide staff in self harm care, there is limited evidence of this happening in practice. The lack of literature including paramedics suggests a gap in our understanding about care for self harm patients. This gap warrants greater attention in order to improve care for patients who self harm in their first point of contact. 


\title{
Appendix J
}

Rees, N. Rapport, F. Snooks, H. (2015) Perceptions of paramedics and emergency staff about the care they provide to people who Self Harm: Constructivist Metasynthesis of the qualitative literature. Journal of Psychosomatic Research. 78: 529-535

\begin{abstract}
Objective: Presentations of self-harm to paramedic and emergency staff are increasing, and despite being the first professionals encountered, patients who self-harm report the quality of care and attitudes from these staff are unsatisfactory. Understanding this care may provide opportunities to improve services. The aim of this study is to enhance knowledge building and theory generation in order to develop practice and policy through a metasynthesis of qualitative research relating to perceptions of paramedic and emergency care for people who self-harm.

Methods: The metasynthesis draws on Evolved Grounded Theory Methodology (EGTM). A search was undertaken of CINAHL®, MEDLINE®, OVID $®$ and Psych INFO ${ }^{\circledR}$, and grey literature. Subject headings of 'self-harm' were used alongside key words 'suicide', 'paramedic' 'emergency', 'overdose', 'pre-hospital' mental health, ambulance, perceptions of care, emergency.
\end{abstract}

Results: A total of 1103 papers were retrieved; 12 were finally included. No papers investigated paramedic care for self-harm. The following metaphors emerged: (a) frustration, futility and legitimacy of care; (b) first contact in the pre-hospital environment: talking, immediate and lasting implications of the moral agent; (c) decision making in self-harm: balancing legislation, risk and autonomy; (d) paramedics' perceptions: harnessing professionalism and opportunities to contribute to the care of self-harm.

Conclusion: Paramedics are often the first health professional contact following selfharm yet limited qualitative literature has explored this encounter. Metaphors revealed in this paper highlight challenges in decision making and legislation, also opportunities to improve care through professionalization and tailored education. 


\begin{tabular}{|c|c|c|c|}
\hline \multicolumn{4}{|c|}{$\begin{array}{r}\text { Truncated representation of the coding book for the Meta } \\
\text { within codes. }\end{array}$} \\
\hline \multicolumn{4}{|c|}{ Selective Codes } \\
\hline $\begin{array}{l}\text { Frustration, futility } \\
\text { and legitimacy of } \\
\text { care }\end{array}$ & $\begin{array}{l}\text { First contact in the pre-hospital } \\
\text { environment: talking, immediate } \\
\text { and lasting implications of the } \\
\text { moral agent; }\end{array}$ & $\begin{array}{l}\text { Decision making in self-harr } \\
\text { legislation, risk and autonon }\end{array}$ & to the \\
\hline \multicolumn{4}{|c|}{ Axial Codes } \\
\hline $\begin{array}{l}\text { Frustration } \\
\text { SH less important than } \\
\text { other physical problems } \\
\text { Rationing of time and care. } \\
\text { Competing demands } \\
\text { Insubstantiality of } \\
\text { interventions } \\
\text { Division between } \\
\text { deliberate nature } \\
\text { of SH and acute medical } \\
\text { conditions, } \\
\text { Calls to SH treated more } \\
\text { slowly } \\
\text { SH of less value } \\
\text { Drug-induced, }\end{array}$ & $\begin{array}{l}\text { First contact } \\
\text { pre-hospital environment } \\
\text { First point of contact with those who SH } \\
\text { paramount and has bearing on future } \\
\text { acceptance of care } \\
\text { Negative experience in first contact can } \\
\text { lead to avoiding emergency services } \\
\text { Individual-oriented care } \\
\text { Immediate and lasting implications } \\
\text { Acceptance or rejection of paramedic care } \\
\text { Environment of ED not conducive to } \\
\text { talking/ engaging with people who SH } \\
\text { Trust and confidence } \\
\text { Paramedic criticised for being a social } \\
\text { worker because he would actually sit } \\
\text { down and talk to people following SH } \\
\text { Context, }\end{array}$ & $\begin{array}{l}\text { Challenge in dealing with people SH and refuse care or } \\
\text { transport } \\
\text { Individual-oriented care } \\
\text { autonomy } \\
\text { Dangers } \\
\text { Decision making } \\
\text { Legalisation } \\
\text { SH potentially life threatening } \\
\text { Safety } \\
\text { Different priorities } \\
\text { Patients have a right to refuse care } \\
\text { Once they do, it's a minefield for us, then } \\
\text { Professional judgments } \\
\text { Unique and non-routine situations } \\
\text { Psychiatric patients identified by paramedic's as 'mad } \\
\text { people' and the police called. } \\
\text { Section } 136 \text { of the MHA }\end{array}$ & $\begin{array}{l}\text { Whole systems approac } \\
\text { Paramedic's first healtt } \\
\text { following SH } \\
\text { Context, } \\
\text { Paramedic criticised fo } \\
\text { because he would actua } \\
\text { people following SH } \\
\text { Professional responsibi } \\
\text { paramedic environmen } \\
\text { in private at the scene, } \\
\text { Capturing opportunitie } \\
\text { SH } \\
\text { Multiple sources of inf } \\
\text { Paramedic role not full } \\
\text { care } \\
\text { Individual-oriented car }\end{array}$ \\
\hline
\end{tabular}




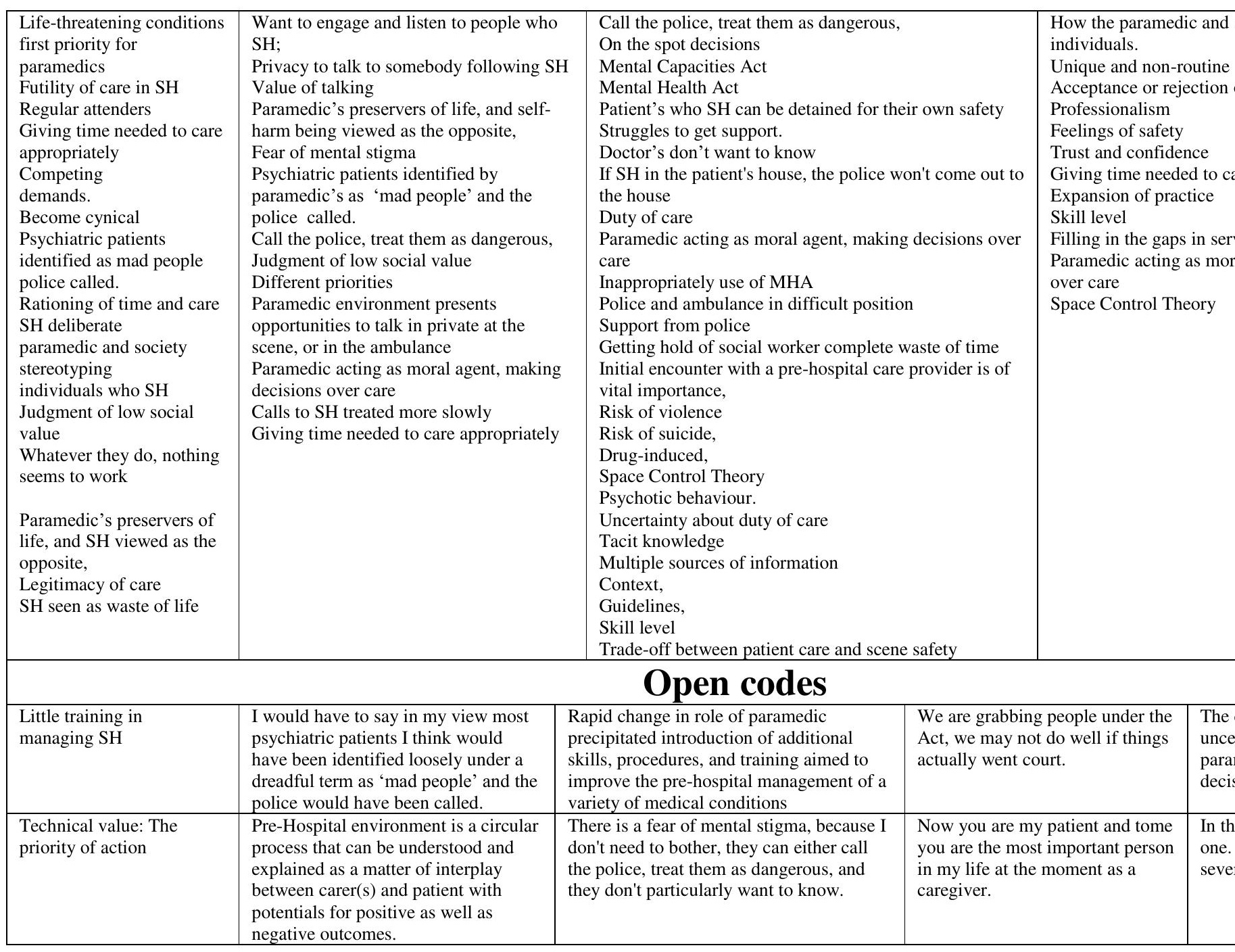




\begin{tabular}{|c|c|c|c|}
\hline & $\begin{array}{l}\text { A call for a patient living in a housing } \\
\text { project known to them, the team } \\
\text { complains about being given another } \\
\text { lousy case and starts driving to the } \\
\text { call without much conviction. On } \\
\text { arrival they find a patient almost } \\
\text { unconscious. Upon seeing this, the } \\
\text { paramedics pick up their pace rapidly } \\
\text { administering Naloxone (a drug that } \\
\text { reverses the effects of overdose). The } \\
\text { patient regains consciousness and } \\
\text { starts breathing normally. } \\
\text { The team recognizes that the patient } \\
\text { has probably overdosed on drugs and } \\
\text { she is placed in the ambulance and } \\
\text { transferred to the hospital without any } \\
\text { display of empathy. }\end{array}$ & $\begin{array}{l}\text { I was criticised for being a social worker } \\
\text { because I would actually sit down and talk } \\
\text { to them. I think it really depends on how } \\
\text { the paramedic and society stereotypes } \\
\text { individuals. }\end{array}$ & $\begin{array}{l}\text { There are ten of us in the room } \\
\text { and obviously we have all got } \\
\text { different ideas if the patient meets } \\
\text { the criteria. }\end{array}$ \\
\hline $\begin{array}{l}\text { I don't think they realise } \\
\text { how potentially life } \\
\text { potentially life threatening } \\
\text { it is. }\end{array}$ & $\begin{array}{l}\text { One girl often takes a lot of } \\
\text { Paracetamol. When she comes in she } \\
\text { has Parvolex treatment every time- } \\
\text { you know, the antidote. I mean, you } \\
\text { are just doing the same thing } \\
\text { time and time again ... working } \\
\text { inhere, I have become cynical-you } \\
\text { just get sick of it. }\end{array}$ & $\begin{array}{l}\text { Transportation or Care, } \\
\text { Acceptance or Rejection, } \\
\text { Participation or Exclusion, } \\
\text { Giving oneself over or calling the care in } \\
\text { question, } \\
\text { The Emergency Ward - Relief or Negative } \\
\text { contrast. }\end{array}$ & $\begin{array}{l}\text { We are probably under equipped } \\
\text { in so far as what we are doing } \\
\text { under the Mental } \\
\text { Health Act is putting us into a } \\
\text { legal realm as such, as we are } \\
\text { grabbing people under } \\
\text { the Act, we may not do well if } \\
\text { things actually went court. }\end{array}$ \\
\hline $\begin{array}{l}\text { Skills in Mental health } \\
\text { ranked higher than skills } \\
\text { and knowledge from } \\
\text { recognised emergency care } \\
\text { courses such as; Pre } \\
\text { Hospital Trauma Life } \\
\text { Support (PHTLS), } \\
\text { Advanced Life Support } \\
\text { (ALS), Pre Hospital } \\
\text { Paediatric Life Support } \\
\text { (PHPLS). }\end{array}$ & $\begin{array}{l}\text { They lifted me on to the stretcher. I'd } \\
\text { close my eyes and felt how well off I } \\
\text { was at the moment. The carers were } \\
\text { wonderful. They listened to me, what } \\
\text { I had strength enough to say. What I } \\
\text { thought was best, what I wanted and } \\
\text { what we should do next, because I } \\
\text { knew my own body. They saw what } \\
\text { they saw but I knew my body }\end{array}$ & $\begin{array}{l}\text { We are probably under equipped in so far } \\
\text { as what we are doing under the Mental } \\
\text { Health Act is putting us into a legal realm } \\
\text { as such, as we are grabbing people under } \\
\text { the Act, we may not do well if things } \\
\text { actually went court }\end{array}$ & $\begin{array}{l}\text { Empathic listening and talking } \\
\text { have key therapeutic benefits }\end{array}$ \\
\hline $\begin{array}{l}\text { There are ten of us in the } \\
\text { room and obviously we } \\
\text { have all got different ideas } \\
\text { [about] if the patient meets } \\
\text { the criteria }\end{array}$ & $\begin{array}{l}\text { You feel you can't give patients the } \\
\text { time that is needed' because of } \\
\text { competing demands. }\end{array}$ & $\begin{array}{l}\text { unsure of the relationship between self- } \\
\text { laceration and both mental illness and risk } \\
\text { of suicide. }\end{array}$ & $\begin{array}{l}\text { If they say, 'I'm not going', we } \\
\text { have no power to take them. We'd } \\
\text { be done for } \\
\text { assault. Either way, you're } \\
\text { screwed, you are. At the end of } \\
\text { the day, they have a right, } \\
\text { their own rights, to refuse. Once } \\
\text { they do, it's a minefield for us, } \\
\text { then. }\end{array}$ \\
\hline
\end{tabular}




\begin{tabular}{|c|c|c|c|}
\hline $\begin{array}{l}\text { Intellectual value: the } \\
\text { attraction of complexity }\end{array}$ & $\begin{array}{l}\text { Interplay between carer(s) and patient } \\
\text { with potentials for positive as well as } \\
\text { negative outcomes. }\end{array}$ & Medical and surgical value & $\begin{array}{l}\text { I became frustrated ... instead of } \\
\text { taking me directly to the hospital } \\
\text { they started fixing thing ... . so I } \\
\text { told them to hurry up. }\end{array}$ \\
\hline $\begin{array}{l}\text { I find it frustrating that I } \\
\text { can't help ... I know of } \\
\text { kids that have been here- } \\
\text { my colleagues have said } \\
\text { that have been regular } \\
\text { attenders-self-harm-it's } \\
\text { frustrating... I mean that is } \\
\text { the frustrating part of it. }\end{array}$ & $\begin{array}{l}\text { Experienced increased job satisfaction } \\
\text { and skills development. }\end{array}$ & $\begin{array}{l}\text { Unclear how mental } \\
\text { illness, whether or not it is classified as an } \\
\text { emergency, is recognised, managed or } \\
\text { represented in the field. }\end{array}$ & $\begin{array}{l}\text { Sessions available on specific } \\
\text { mental health diseases and } \\
\text { symptoms/management. More } \\
\text { information on risk assessment of } \\
\text { suicide/aggression. Information } \\
\text { on negotiation with patient, while } \\
\text { assessing to reduce the need for } \\
\text { SAPOL section } 23 \text { [legal } \\
\text { detention]. }\end{array}$ \\
\hline $\begin{array}{l}\text { When you've got a } \\
\text { department or ward full of } \\
\text { severe asthma, meningitis, } \\
\text { etc and then you've got a } \\
\text { couple of young girls who } \\
\text { have taken a cocktail of } \\
\text { things ... They cannot ... } \\
\text { with our current resources . } \\
\text {. . be looked after in the } \\
\text { same way. }\end{array}$ & $\begin{array}{l}\text { Complexity } \\
\text { of the decision-making process }\end{array}$ & $\begin{array}{l}\text { In contrast to other healthcare providers, } \\
\text { paramedics do not have predetermined } \\
\text { work areas, but accept the location where } \\
\text { they find a patient as their working area } \\
\text { and adapt themselves and the environment } \\
\text { around the patient accordingly, and it is } \\
\text { this adaptation he suggests enables } \\
\text { provision patient care }\end{array}$ & $\begin{array}{l}\text { The doctor doesn't want to know, } \\
\text { usually. orwe'll get in touch with } \\
\text { the police If it's in the patient's } \\
\text { house, the police won't come out } \\
\text { to the house, they won't come } \\
\text { across the threshold, very often. }\end{array}$ \\
\hline $\begin{array}{l}\text { The team recognizes that } \\
\text { the patient has probably } \\
\text { overdosed on drugs and } \\
\text { she is placed in the } \\
\text { ambulance and transferred } \\
\text { to the hospital without any } \\
\text { display of empathy. }\end{array}$ & $\begin{array}{l}\text { Nursing services are at the core of the } \\
\text { ED, and are usually the first } \\
\text { healthcare professionals with whom a } \\
\text { self harm patient comes into contact, } \\
\text { providing triage, first aid and } \\
\text { psychological support. }\end{array}$ & $\begin{array}{l}\text { Think safety is of paramount concern with } \\
\text { mental illness, possibly more so than with } \\
\text { other complaints and certainly your } \\
\text { preparation and awareness of potential or } \\
\text { unpredictable situations is heightened } \\
\text { when attending a case with mental health } \\
\text { issues. }\end{array}$ & $\begin{array}{l}\text { The roles of paramedics and } \\
\text { contributions they can make to } \\
\text { the care of the mentally ill } \\
\text { in the wider continuum of health } \\
\text { care have not been fully } \\
\text { recognised. }\end{array}$ \\
\hline $\begin{array}{l}\text { This department isn't } \\
\text { conducive to being able to } \\
\text { talk to them in private. you } \\
\text { have got different } \\
\text { priorities. }\end{array}$ & $\begin{array}{l}\text { Patient's capacity to make decisions } \\
\text { and how this is interpreted }\end{array}$ & $\begin{array}{l}\text { The caregiver's ability to inspire } \\
\text { immediate confidence and trust is of vital } \\
\text { importance, otherwise there is an obvious } \\
\text { risk that the patient will distrust the care } \\
\text { situation and a vicious circle of mistrust } \\
\text { emerges". }\end{array}$ & $\begin{array}{l}\text { The busy quality of such wards } \\
\text { (busyness) } \\
\text { How this group of clients impede } \\
\text { the busy quality } \\
\text { Strategies nurses use to cope with } \\
\text { the difficulties }\end{array}$ \\
\hline
\end{tabular}


Appendix L

Rees N, Porter A, Rapport F, Hughes S, John A (2018) Paramedics' perceptions of the care they provide to people who self-harm: A qualitative study using evolved grounded theory methodology. PLoS ONE 13(10): e0205813. https://doi.org/10.1371/journal.pone.0205813

\begin{abstract}
Background: Self-harm (SH) accounts for over 5\% of the workload of emergency ambulance services, and therefore Paramedics are often the first health professional in contact with people who SH. The authors of this paper have reported elsewhere the significant gaps in our understandings which exist surrounding this early care interaction, and some of the challenges paramedics and opportunities in Pandemic care for people who SH. This study aimed to explore paramedics' perceptions of caring for those who SH using Evolved Grounded Theory Methodology.

Methods: This study took place between 2014-2016 in one UK ambulance service covering a population of three million people. Semi structured interviews were conducted, purposively sampling paramedics until saturation was reached. Interviews were recorded, transcribed verbatim, and coded through open, axial, and selective levels of coding, identifying the Basic Social Process (BSP) and developing a Grounded Theory. A second researcher (SH) independently reviewed early results, which were also member-checked with participants.

Results: Eleven paramedics were interviewed. The following six categories emerged: Context; Judgements and values; Isolation and system failure; Managing risk; Competence at the boundary of mental and physical health needs; Professional, legal and ethical tensions. The BSP Decision making in a context of risk was identified. The final Grounded Theory that emerged was one of 'Wicked Complexity of paramedic care for people who $\mathrm{SH}$, which includes usual factors such as tiredness and frequent callers, heightened factors including lack of support and pathways, and factors specific to $\mathrm{SH}$ such assessing mental health and suicide risk.
\end{abstract}

Conclusions: This study builds on a very small body of literature to have explored paramedic care for people who SH and has found that this care interaction provides uniquely complex challenges. The multiple influences within the categories defined in this study need considering conjointly when making improvements to care. 


\section{Truncated representation of the coding book, and the interrelationship within codes.}

The Evolved Grounded Theory: Wicked Complexity in Paramedics' care provided to people who Self-h

\section{Basic Social Process: Decision making in a context of risk}

\section{Selective Codes}

\begin{tabular}{|c|c|c|c|c|c|}
\hline $\begin{array}{l}\text { Isolation and } \\
\text { system failures }\end{array}$ & Managing risk & $\begin{array}{l}\text { Judgements and } \\
\text { values }\end{array}$ & $\begin{array}{l}\text { Competence at the } \\
\text { boundary of mental } \\
\text { and physical health } \\
\text { needs }\end{array}$ & $\begin{array}{l}\text { Professional, } \\
\text { legal and ethical } \\
\text { tensions }\end{array}$ & Context \\
\hline \multicolumn{6}{|c|}{ Axial Codes } \\
\hline $\begin{array}{l}\text { First contact } \\
\text { Poor standard of care } \\
\text { offered by } \\
\text { paramedics } \\
\text { Physical Versus } \\
\text { Mental Health } \\
\text { problems } \\
\text { Professional role in } \\
\text { mental health } \\
\text { Technical skills } \\
\text { versus knowledge } \\
\text { and decision making } \\
\text { Time after hand over } \\
\text { Desire to help } \\
\text { Knowledge and } \\
\text { understanding } \\
\text { Judgement of risk } \\
\end{array}$ & $\begin{array}{l}\text { Judgement of risk } \\
\text { Role of alcohol } \\
\text { First contact } \\
\text { Physical Versus Mental } \\
\text { Health problems } \\
\text { Professional role in mental } \\
\text { health } \\
\text { Technical skills versus } \\
\text { knowledge and decision } \\
\text { making } \\
\text { Knowledge and } \\
\text { understanding } \\
\text { Training and education } \\
\text { Mostly minor } \\
\text { Sometimes goes wrong } \\
\text { Safety } \\
\text { Of others }\end{array}$ & $\begin{array}{l}\text { Role of alcohol } \\
\text { First contact } \\
\text { Defending patients: } \\
\text { Relationships built up } \\
\text { with regulars (patients) } \\
\text { Empathy } \\
\text { Desire to help } \\
\text { Repetition } \\
\text { A performance for others } \\
\text { Mostly minor } \\
\text { Sometimes goes wrong } \\
\text { Waste of time } \\
\text { Escalating risk } \\
\text { Frustrating } \\
\text { Talking } \\
\text { Busyness } \\
\text { Guarding patients } \\
\end{array}$ & $\begin{array}{l}\text { Role of alcohol } \\
\text { First contact } \\
\text { Poor standard of care } \\
\text { offered by paramedics } \\
\text { Technical skills versus } \\
\text { knowledge and decision } \\
\text { making } \\
\text { Physical Versus Mental } \\
\text { Health problems } \\
\text { Technical skills versus } \\
\text { knowledge and decision } \\
\text { making } \\
\text { Knowledge and } \\
\text { understanding } \\
\text { Access to training } \\
\text { Training and education } \\
\text { Judgement of risk }\end{array}$ & $\begin{array}{l}\text { Sometimes } \\
\text { surreptitious means } \\
\text { Relationships with } \\
\text { Police } \\
\text { Role of alcohol } \\
\text { First contact } \\
\text { Defending patients: } \\
\text { Professional role in } \\
\text { mental health } \\
\text { Knowledge and } \\
\text { understanding } \\
\text { Access to training } \\
\text { Training and education } \\
\text { Judgement of risk } \\
\text { Safety } \\
\text { Of others } \\
\text { Frustrating }\end{array}$ & $\begin{array}{l}\text { Role of alcohol } \\
\text { First contact } \\
\text { Technical skills ve } \\
\text { decision making } \\
\text { Technical skills ve } \\
\text { decision making } \\
\text { Time after hand o } \\
\text { Empathy } \\
\text { Judgement of risk } \\
\text { Repetition } \\
\text { A performance for } \\
\text { Mostly minor } \\
\text { Crying for help } \\
\text { Sometimes goes u } \\
\text { Waste of time } \\
\text { Talking } \\
\text { Busyness }\end{array}$ \\
\hline
\end{tabular}




\begin{tabular}{|c|c|c|c|c|c|c|}
\hline $\begin{array}{l}\text { Repetition } \\
\text { Crying for help } \\
\text { Guarding patients } \\
\text { Tazers } \\
\text { Role of alcohol } \\
\text { Ways and means } \\
\text { Overplaying risk } \\
\text { Frustrating } \\
\text { Competence } \\
\text { SH viewed as Mental } \\
\text { Health problem } \\
\text { Law } \\
\text { Appropriate care } \\
\text { options } \\
\text { Rural v urban }\end{array}$ & $\begin{array}{l}\text { Escalating risk } \\
\text { Guarding patients } \\
\text { Competence } \\
\text { SH viewed as Mental } \\
\text { Health problem } \\
\text { Fear of litigation } \\
\text { Law } \\
\text { Detaining is sometimes } \\
\text { quicker: } \\
\text { Risk of suicide } \\
\text { Ways and means }\end{array}$ & $\begin{array}{l}\text { Poor attitudes; not me } \\
\text { but some of my } \\
\text { colleagues } \\
\text { Time In the back one to } \\
\text { one } \\
\text { SH viewed as Mental } \\
\text { Health problem } \\
\text { Mostly minor } \\
\text { Scale of SH }\end{array}$ & $\begin{array}{l}\text { Mostly minor } \\
\text { Crying for help } \\
\text { Sometimes goes wrong } \\
\text { Competence } \\
\text { SH viewed as Mental } \\
\text { Health problem } \\
\text { Fear of litigation } \\
\text { Appropriate care options } \\
\text { Risk of suicide } \\
\text { Ways and means } \\
\text { Overplaying risk }\end{array}$ & \multicolumn{2}{|c|}{$\begin{array}{l}\text { Busyness } \\
\text { Guarding patients } \\
\text { Poor attitudes; not me } \\
\text { but some of my } \\
\text { colleagues } \\
\text { Competence } \\
\text { SH viewed as Mental } \\
\text { Health problem } \\
\text { Fear of litigation } \\
\text { Detention } \\
\text { Law } \\
\text { Detaining is sometimes } \\
\text { quicker: } \\
\text { Detaining is sometimes } \\
\text { quicker: } \\
\text { Risk of suicide } \\
\text { Tazers } \\
\text { Ways and means } \\
\text { Overplaying risk } \\
\end{array}$} & $\begin{array}{l}\text { Guarding patients } \\
\text { Poor attitudes; not } \\
\text { colleagues } \\
\text { Time In the back } \\
\text { SH viewed as Mer } \\
\text { Rural v urban } \\
\text { In the back } \\
\text { A call for help son } \\
\text { Appropriate care c } \\
\text { Risk of suicide } \\
\text { Mostly minor } \\
\text { Scale of SH }\end{array}$ \\
\hline & & & \multicolumn{4}{|l|}{ pen codes } \\
\hline $\begin{array}{l}\text { Safety } \\
\text { Of others } \\
\text { Potential of a greater } \\
\text { threat to the patient } \\
\text { or threat towards you } \\
\text { as well. concern, as } \\
\text { always, with these } \\
\text { individuals, that they } \\
\text { will go on to cause } \\
\text { serious injury. I } \\
\text { ascertained that she } \\
\text { wasn't a danger to } \\
\text { anybody. quite } \\
\text { aggressive and } \\
\text { angry, and then they } \\
\text { could be harm to } \\
\text { other people. }\end{array}$ & \multicolumn{2}{|c|}{$\begin{array}{l}\text { Escalating risk } \\
\text { sort of like progressive, so it can sort of start off as self } \\
\text { harming and then become more intentional. Our main } \\
\text { concern has to be that, you know, do they then move from } \\
\text { that situation where perhaps they've caused themselves } \\
\text { some harm, but not real harm with the possibility of } \\
\text { ending their life. will they then go on to continue to do so } \\
\text { until they actually, you know, succeed in attempting their } \\
\text { own, taking their own lives. started with small } \\
\text { lacerations to the wrists, the lacerations got bigger, and } \\
\text { the last time I attended she'd actually severed down to } \\
\text { her carotid artery. people do progress for whatever } \\
\text { reason. started off with minor lacerations, and the } \\
\text { lacerations have got bigger, and bigger, and bigger. they } \\
\text { could move up a stage and do something a lot worse next } \\
\text { time. Today it could be a scratch, tomorrow it could be a } \\
\text { deeper wound that's nicked an artery or nicked a tendon. }\end{array}$} & \multicolumn{2}{|c|}{$\begin{array}{l}\text { Training and education } \\
\text { we don't have the training, due to our } \\
\text { limited training. I would prefer, erm, I } \\
\text { would like paramedics, the world seems } \\
\text { to be full of statutory and mandatory } \\
\text { training, You know, the training simply } \\
\text { isn't there to give you any } \\
\text { understanding. You have to--, you } \\
\text { formulate your care and your } \\
\text { understanding of such people from your } \\
\text { own sort of background, your own } \\
\text { thought process, your own empathy } \\
\text { towards them, as opposed to, you know, } \\
\text { having some training and understanding } \\
\text { in understanding why people self harm. } \\
\text { Well, there's no specific training, they } \\
\text { give appropriate care physically, } \\
\text { medically, and try their best emotionally } \\
\text { I think }\end{array}$} & \multicolumn{2}{|c|}{$\begin{array}{l}\text { Waste of time } \\
\text { disdain pretty much, because } \\
\text { you're wasting my time actua } \\
\text { they're a bloody nuisance. st } \\
\text { wasting our time }\end{array}$} \\
\hline
\end{tabular}




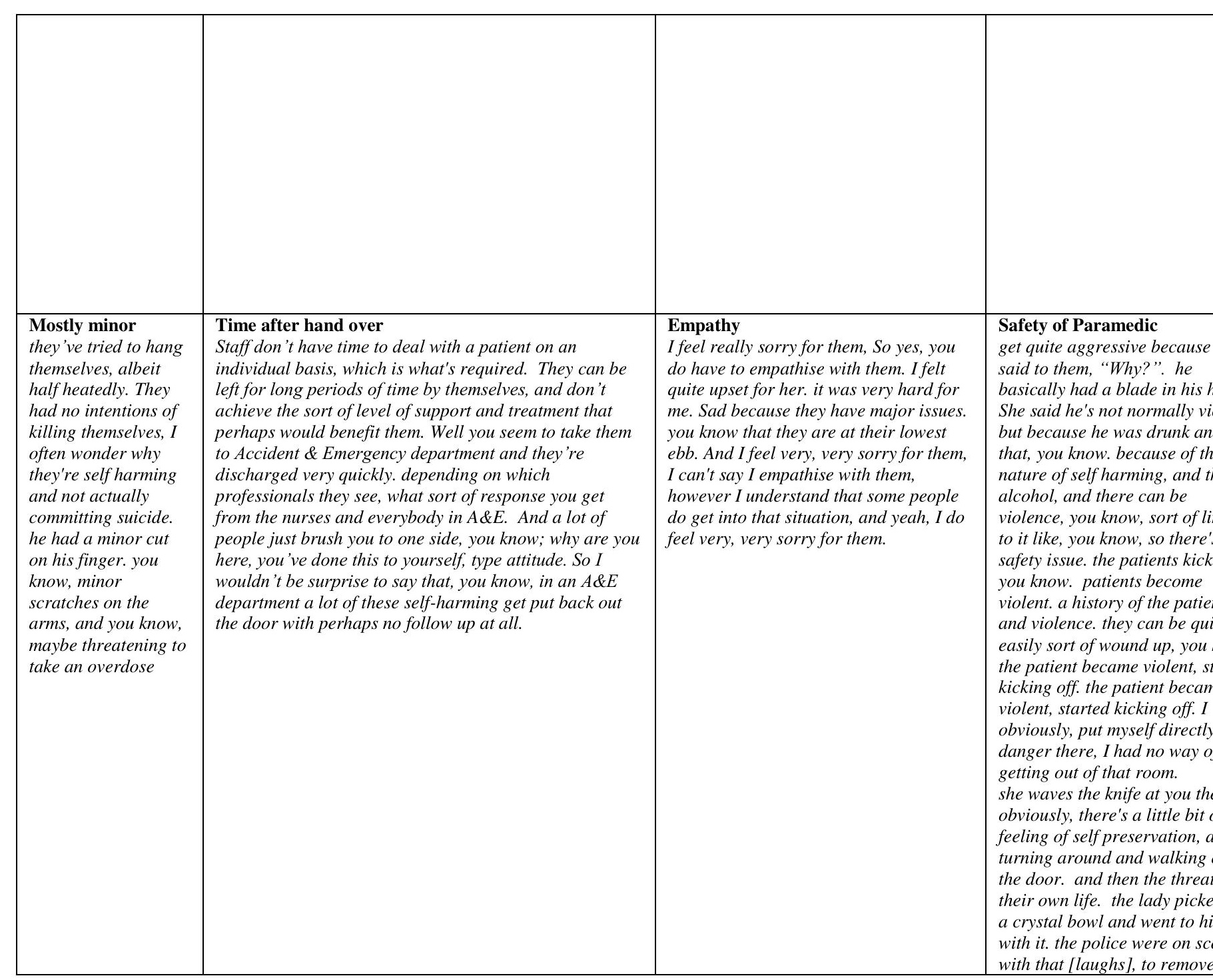




\begin{tabular}{|c|c|c|c|}
\hline & & & $\begin{array}{l}\text { knife, etc. But then he come } \\
\text { the ambulance and he'd had } \\
\text { knife hidden in his }\end{array}$ \\
\hline $\begin{array}{l}\text { Desire to help } \\
\text { I would like to help } \\
\text { them really. we } \\
\text { wanted to sort of help } \\
\text { her and it was very } \\
\text { hard to tell whether } \\
\text { she wanted it or not, }\end{array}$ & $\begin{array}{l}\text { Access to training } \\
\text { through my time at university this subject was broached } \\
\text { a few times, once by an ethicist, a professor in ethics. } \\
\text { Whereas we spoke to a lawyer, we had a lecture from a } \\
\text { lawyer and the same scenario was put to that lawyer, } \\
\text { after the ethics lecture. I certainly haven't had enough } \\
\text { training to be able to provide these people with the } \\
\text { instant support they require at that point. We touch upon } \\
\text { mental health issues in university. So mental health crisis } \\
\text { teams would be great, you know, if we could have some } \\
\text { experiences with that. when I was in university when I } \\
\text { chose a subject similar to that to do some research on } \\
\text { and I realised that my perceptions of self harmers were } \\
\text { not quite correct. One thing I learned from higher } \\
\text { education is that I don't believe everything I read } \\
\text { whereas I think I did before, but that taught me that a lot } \\
\text { of people will make statements and because they're } \\
\text { written down there's not strictly any research behind it. } \\
\text { the difficulties are managing patients with psychiatric } \\
\text { problems to start with with the minimum training that } \\
\text { we've had. through the university, the university was--, } \\
\text { erm, was quite helpful. We did quite a lot of psychology } \\
\text { during the course so we did cover certain aspects of it. } \\
\text { However, I didn't feel that self harm and suicide we } \\
\text { covered a huge amount on. We had maybe an hour or } \\
\text { two lectures on it. I have some knowledge on the } \\
\text { background through the university. }\end{array}$ & $\begin{array}{l}\text { Knowledge and understanding } \\
\text { the involuntary section, I can't remember } \\
\text { the numbers of them. that a lot of people } \\
\text { will make statements and because } \\
\text { they're written down there's not strictly } \\
\text { any research behind it. I think one thing } \\
\text { that ambulance staff are pretty good at } \\
\text { is trying to make sense out of the } \\
\text { situation. Because nobody understands } \\
\text { it. if we don't understand it we can't } \\
\text { treat them correctly }\end{array}$ & $\begin{array}{l}\text { A performance for others } \\
\text { people who make a big displc } \\
\text { going to commit suicide, peo, } \\
\text { that stand on the bridge and } \\
40 \text { police officers who think } \\
\text { they're all heroes around the } \\
\text { never going to actually do it } \\
\text { intentionally. some do it for, } \\
\text { know, sort of attention. sort } \\
\text { show like. enough to cause a } \\
\text { an attention. there'll be a bit } \\
\text { personality performance in ti } \\
\text { hospital, and they like to gain } \\
\text { attention. }\end{array}$ \\
\hline $\begin{array}{l}\text { Professional role in } \\
\text { mental health } \\
\text { I think a paramedic's } \\
\text { role is whatever's } \\
\text { needed, to me as a } \\
\text { lay professional, } \\
\text { your professional } \\
\text { practice, down to us } \\
\text { as health care } \\
\text { professionals, I am } \\
\text { duty bound, prior } \\
\text { HCPC registration, } \\
\text { fearful of losing their } \\
\text { professional status, } \\
\text { as professionals we }\end{array}$ & $\begin{array}{l}\text { Relationships built up with regulars (patients) } \\
\text { I've been to him dozens of times. I say, you're regular } \\
\text { one where, you know, they're calling you 'cause they've } \\
\text { self harmed, they know exactly what the system is. people } \\
\text { call us out and they say, "Well thank you for coming." } \\
\text { consistently seeing this type of call, over and over and } \\
\text { over again, it's the same thing, you know you're probably } \\
\text { going to see them again. I was having her as a regular } \\
\text { patient. we were on first name terms, I'd go in and I'd } \\
\text { say, "Oh fuck Lucy, what have you done now?", I mean } \\
\text { this guy Darren Yeah, I know Chris, I'm sorry, How } \\
\text { much blood have you lost Lucy?" "Oh a shed load." } \\
\text { "Okay, come on sweet cheeks." I think it helped Lucy } \\
\text { feel not so bad about herself and what she'd done. all of } \\
\text { a sudden it went to hell for her again. And I saw her }\end{array}$ & $\begin{array}{l}\text { Physical Versus Mental Health } \\
\text { problems } \\
\text { I can do very little for you medically } \\
\text { wise, psyche patients you're less inclined } \\
\text { to start messing around with them, } \\
\text { you're less inclined to sort of like } \\
\text { intervene with someone with } \\
\text { psychological issues, you could actually } \\
\text { miss a medical condition. there's nothing } \\
\text { to treat, There's no wound, there's no } \\
\text { medical condition that you have, you } \\
\text { know, you've got no tools, this is } \\
\text { psychological issue, like you know, and } \\
\text { there's very little you can do. if you've } \\
\text { got someone with a respiratory }\end{array}$ & $\begin{array}{l}\text { Sometimes goes wrong } \\
\text { I don't believe for a minute th } \\
\text { they intended to take their on } \\
\text { at that stage, but have, they } \\
\text { done so, if you can do it by e } \\
\text { just grabbed the closest thing } \\
\text { hand, not realising by taking } \\
\text { substance they've taken that } \\
\text { is actually going to have a se } \\
\text { effect, and probably going to } \\
\text { their life. And on all three } \\
\text { occasions the patient has act } \\
\text { died later on. I don't think he } \\
\text { intended to harm himself eve } \\
\text { though the act would have ta }\end{array}$ \\
\hline
\end{tabular}




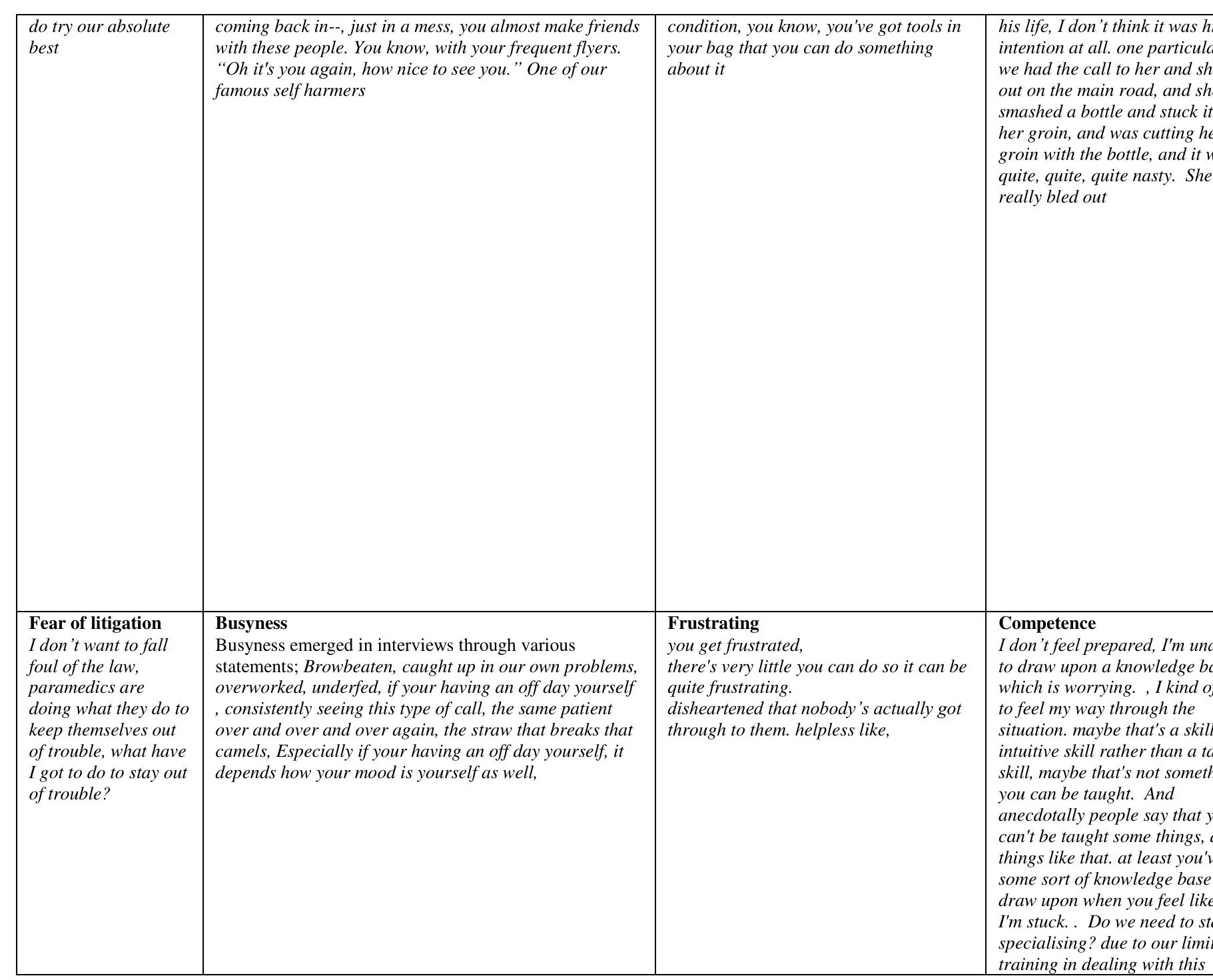




\begin{tabular}{|c|c|c|c|}
\hline & & & $\begin{array}{l}\text { always look at the worst case } \\
\text { scenario }\end{array}$ \\
\hline $\begin{array}{l}\text { Detention } \\
\text { sectioning somebody, } \\
\text { a place of safety, the } \\
\text { police could arrest } \\
\text { him, entrapment, } \\
\text { force this gentleman } \\
\text { into hospital, save } \\
\text { that person's life by } \\
\text { force if necessary, } \\
\text { forcibly intervening, } \\
\text { restrain people who } \\
\text { are intent on self } \\
\text { harming themselves. }\end{array}$ & $\begin{array}{l}\text { Poor attitudes; not me but some of my colleagues } \\
\text { the biggest barrier is the person themselves, as in the } \\
\text { paramedic themselves. a lot of my colleagues will say } \\
\text { that, you know, that they are wasting our time. the kind } \\
\text { of attitudes by... a quantity of ambulance staff is that, } \\
\text { "We're out to this idiot again.", we've got a number of } \\
\text { people that are not very understanding. a colleague I'd } \\
\text { been working with hasn't been so understanding. we've } \\
\text { got a lot of people that don't understand, I have the } \\
\text { desires to do what's right--, what's best for the patients, } \\
\text { but I don't see that in every paramedic. the medic at the } \\
\text { time has not treated it well. the colleague I was with was } \\
\text { quite aggressive towards the patient, not a caring sort of } \\
\text { attitude. }\end{array}$ & $\begin{array}{l}\text { Guarding patients } \\
\text { My problem is to worry about my } \\
\text { patients, which I do and I'm not } \\
\text { interested in your eight minute response, } \\
\text { that's your problem. Reference } 1 \text { - } \\
\text { O.77\% Coverage } \\
\text { I have on occasions, you know, got my } \\
\text { colleague to stop the vehicle if we've got } \\
\text { a relative, if I think that there's an } \\
\text { increase in distress in the patient, and } \\
\text { put the relative in the front, and that's } \\
\text { generally when we find that your patient } \\
\text { will talk to you more about the problems } \\
\text { they find. They just don't want } \\
\text { friends/family knowing what they're } \\
\text { going through. } \\
\text { And when we drove to the hospital and } \\
\text { we report clear, he asked what was that } \\
\text { about. I said, "I didn't like the silence } \\
\text { in the back of my ambulance." "Well I } \\
\text { was in the back," "I don't care that you } \\
\text { were in the back, if you can't talk to your } \\
\text { patient you'll come out the back every } \\
\text { time." } \\
\text { I took him into the office and explained it } \\
\text { to him that not to talk to a patient was } \\
\text { unacceptable. }\end{array}$ & $\begin{array}{l}\text { Talking } \\
\text { Absolutely back right off, you } \\
\text { don't want to talk to me that' } \\
\text { That's your prerogative, you } \\
\text { want to talk, don't talk, } \\
\text { occasionally that will get the } \\
\text { talking. just conversation aro } \\
\text { other things I didn't really kn } \\
\text { what to say, I didn't know wh } \\
\text { were the right things to say } \\
\text { you worry about the woman } \\
\text { suddenly saying, "No, I don, } \\
\text { want to go," and, "Stop the } \\
\text { ambulance." I've had people } \\
\text { badly to, erm, my comments } \\
\text { back of an ambulance, or get } \\
\text { aggressive because I've said } \\
\text { them, "Why?" And people sa } \\
\text { don't want to talk." Absolute } \\
\text { back right off, you don't wan } \\
\text { talk to me that's fine. That's } \\
\text { prerogative, you don't want } \\
\text { talk, don't talk. So we're not } \\
\text { there like a pair of dummies. } \\
\text { don't feel that I'm, I'm pushir } \\
\text { being pushy or whatever. or } \\
\text { ask them stupid questions, or } \\
\text { make some comment about, } \\
\text { love those shoes." [Laughte } \\
\text { "Love those shoes." And }\end{array}$ \\
\hline
\end{tabular}




\begin{tabular}{|c|c|c|c|}
\hline & & & $\begin{array}{l}\text { occasionally that will get the } \\
\text { talking. you know, just } \\
\text { conversation around other th } \\
\text { Occasionally they'll open up. } \\
\text { They're not going to talk to y } \\
\text { change the subject, you knou } \\
\text { talk about something comple } \\
\text { different. I'm never one to eit } \\
\text { sit back and do nothing and } \\
\text { talk to them }\end{array}$ \\
\hline $\begin{array}{l}\text { Support and } \\
\text { appropriate care } \\
\text { Lonely. Concerned, } \\
\text { on occasions We } \\
\text { don't have the } \\
\text { pathways. you're the } \\
\text { clinician on the } \\
\text { scene, you have to } \\
\text { make that decision. It } \\
\text { does make you feel } \\
\text { lonely. I think it's } \\
\text { chronically } \\
\text { underfunded, I don't } \\
\text { think there's enough } \\
\text { expertise readily } \\
\text { available to help } \\
\text { these people you } \\
\text { can't get hold of } \\
\text { mental health } \\
\text { practitioners to be } \\
\text { able to help you, the } \\
\text { lack of advice and } \\
\text { support. The lack of } \\
\text { referral facilities. }\end{array}$ & $\begin{array}{l}\text { In the back } \\
\text { get her out to the ambulance, try and get them into } \\
\text { hospital, get her in the truck, that left me and this person } \\
\text { in the back of the ambulance, kind of crunched up on our } \\
\text { trolley, "Stop the ambulance.", transporting that patient } \\
\text { to hospital. police have come into the ambulance as well, } \\
\text { just driving them into hospital, Just come out to the back } \\
\text { of the ambulance, }\end{array}$ & $\begin{array}{l}\text { Detaining is sometimes quicker: } \\
\text { the easier decision would have been to } \\
\text { force this gentleman into hospital, } \\
\text { To carry out an assessment then that } \\
\text { quite often would take several--, several } \\
\text { hours } \\
\text { I think the easier decision would have } \\
\text { been to force this gentleman into } \\
\text { hospital, certainly. I mean if you wanted } \\
\text { to be lazy about it, you know, that would } \\
\text { have been the easiest thing, you know, } \\
\text { less time we've seen, less head work and } \\
\text { things. }\end{array}$ & $\begin{array}{l}\text { Ways and means } \\
\text { the Ways and Means Act, an } \\
\text { was the ways and means of g } \\
\text { somebody--, and you know, I } \\
\text { quite happily put my hands u } \\
\text { on tape and say that, you kno } \\
\text { I've been in situations where } \\
\text { somebody has deliberately se } \\
\text { harmed and the police have } \\
\text { themselves powerless to do } \\
\text { anything } \\
\text { you know, where they lie in } \\
\text { regards to sectioning someb } \\
\text { or taking somebody } \\
\text { there was some talk about tr. } \\
\text { to get him to go outside whe } \\
\text { police could arrest him beca } \\
\text { he was no longer inside. No } \\
\text { ethicacy of that doesn't sit w } \\
\text { with me, but if the gentleman } \\
\text { going to die from this overdo } \\
\text { then, you know, what's more } \\
\text { important life or ethics? } \\
\text { A cigarette, come outside for } \\
\text { cigarette. So you know, that } \\
\text { using, I don't know, entrapm } \\
\text { something [laughs]. I don't } \\
\text { what the word is. But certai } \\
\text { get him outside under false } \\
\text { pretences, and then once he } \\
\text { outside the property he coulo } \\
\text { arrested by the police 'cause } \\
\text { wasn't in his own property. }\end{array}$ \\
\hline
\end{tabular}




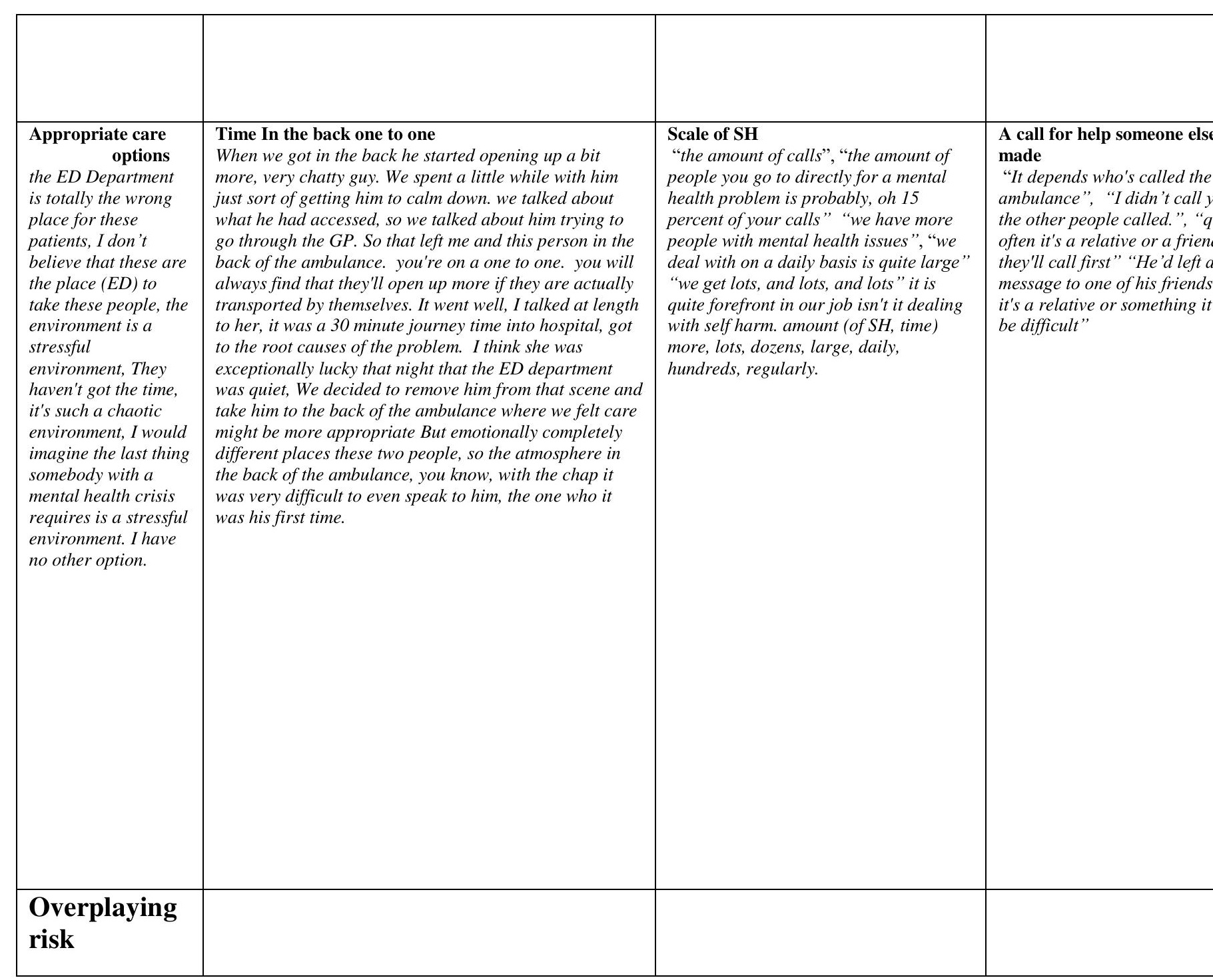




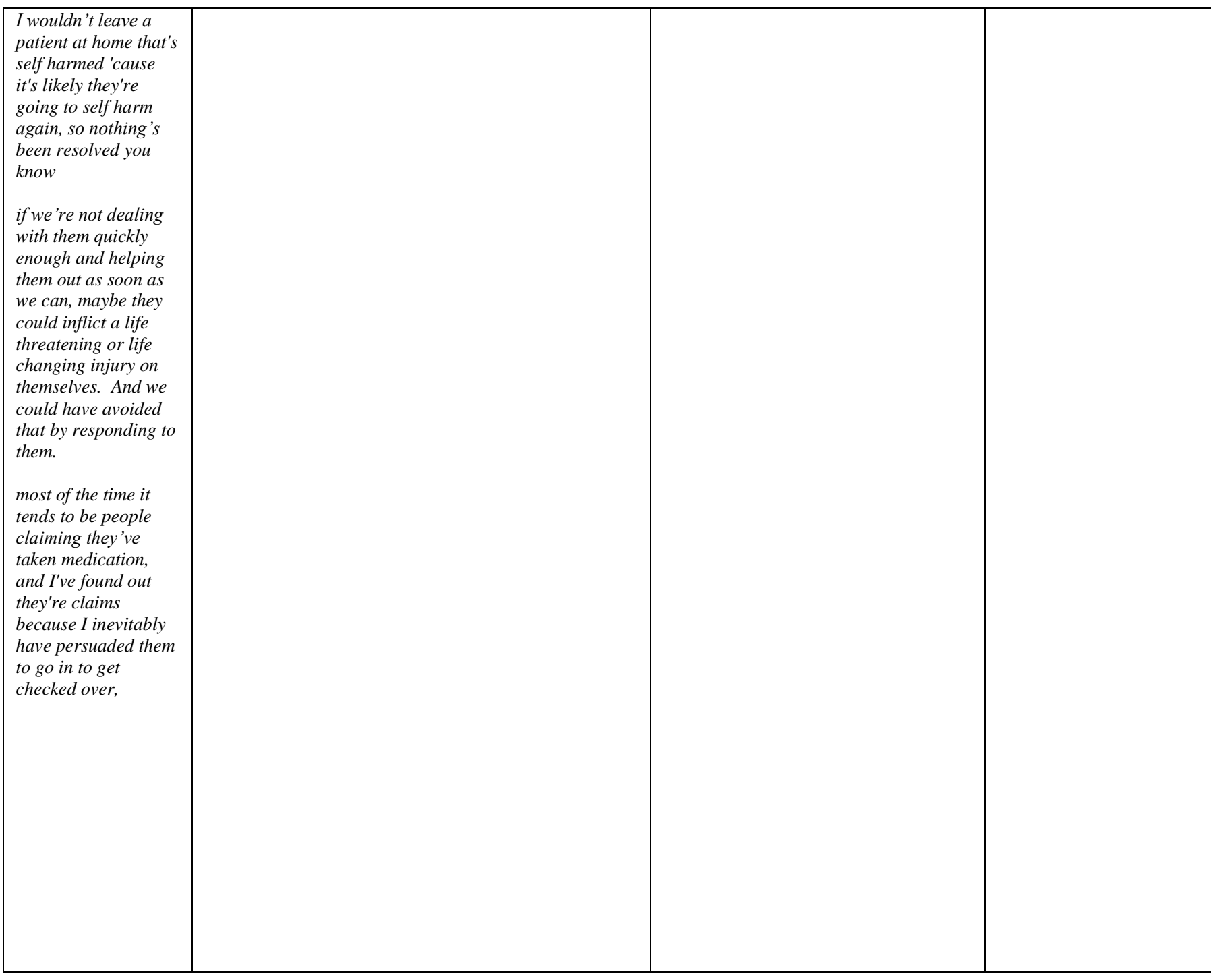




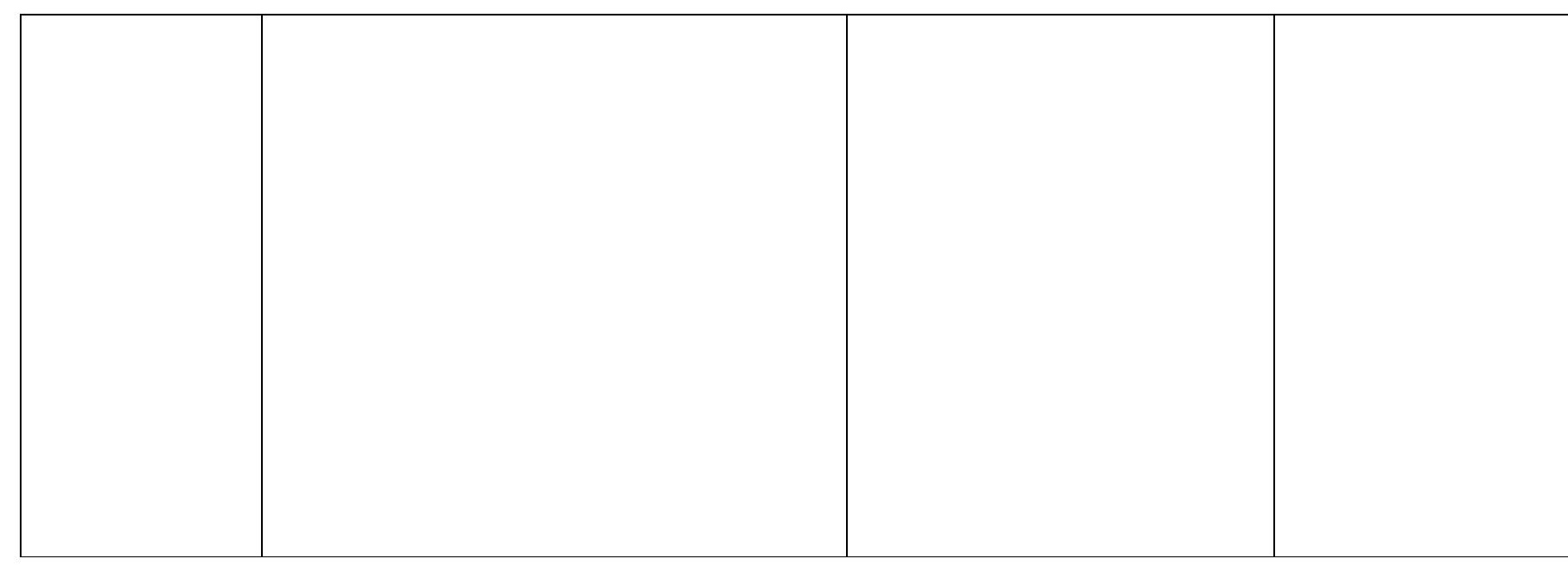


Appendix N

\section{Activities to include meetings, peer reviewed oral and poster presentations at conferences, publications, and supporting position statements.}

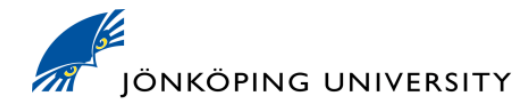

Programme for visit to School of Health Sciences at Jönköping University, $10^{\text {th }}-12^{\text {th }}$ March, 2014

Nigel Rees, Senior Research Lead for the Welsh Ambulance Service, Swansea University Monday $10^{\text {th }}$ March

10.00-11.30 Visit to the School of Health Sciences (HHJ, Hälsohögskolan in Swedish). Karl Hedman, International Relations Manager, School of Health Sciences Place: 5C 419 (4th floor at $\mathrm{HHJ}$ ) side corridor towards the tax office building

12.00-14.00 Lunch at Culture House Spira http://www.kulturhusetspira.se/ with Karl Hedman

14.00-16.00 Research discussions about for example caring for people who self harm in ambulance emergencies in Wales and Stockholm, Sweden, with Karl Hedman writing his PhD at Lund University about emergency calls to the Stockholm emergency medical dispatch centre about suicide attempts and other reasons.

Nigel Rees to present to pre hospital researchers and students: Paramedic care for people who Self Harm

Tuesday $11^{\text {th }}$ March

10.10 Bus nr 200 from the Jönköping bus station to the Ulricehamn bus station arriving 11.10

where Karl is picking you up at the bus station. We then drive to Borås for lunch before visiting the ambulance services.

14.00- Visit to the ambulance station at Borås Hospital Ingemar Sundgren, Education Head Ambulanssjukvården i Södra Älvsborg Samaritvägen 9

Entrance $22 \mathrm{C}$ plan 1

50182 Borås

Phone:

Fax:

E-mail:

About the procedures of the Borås ambulance services and:

- The link between researchers, academics, ambulance services and clinical practice.

- I would also like a discussion on the influence of mental health legislation on the ambulance services

- Alternatives pathways to the emergency department 
16.56-17.51 Ulricehamn-Jönköping bus 200 or alternatively:

17.41-18.36 Ulricehamn-Jönköping bus 200

\section{Wednesday $11^{\text {th }}$ March}

9.00-12.00 Lecture by Nigel Rees Paramedics perceptions of caring for people who Self harm: An Evolved Grounded Theory, in lecture room Ga 334, School of Health Sciences

Contact:

Karl Hedman

Office

Mobile phone

Department of Nursing: Ingalill Gimbler Berglund and Eva Andersson 


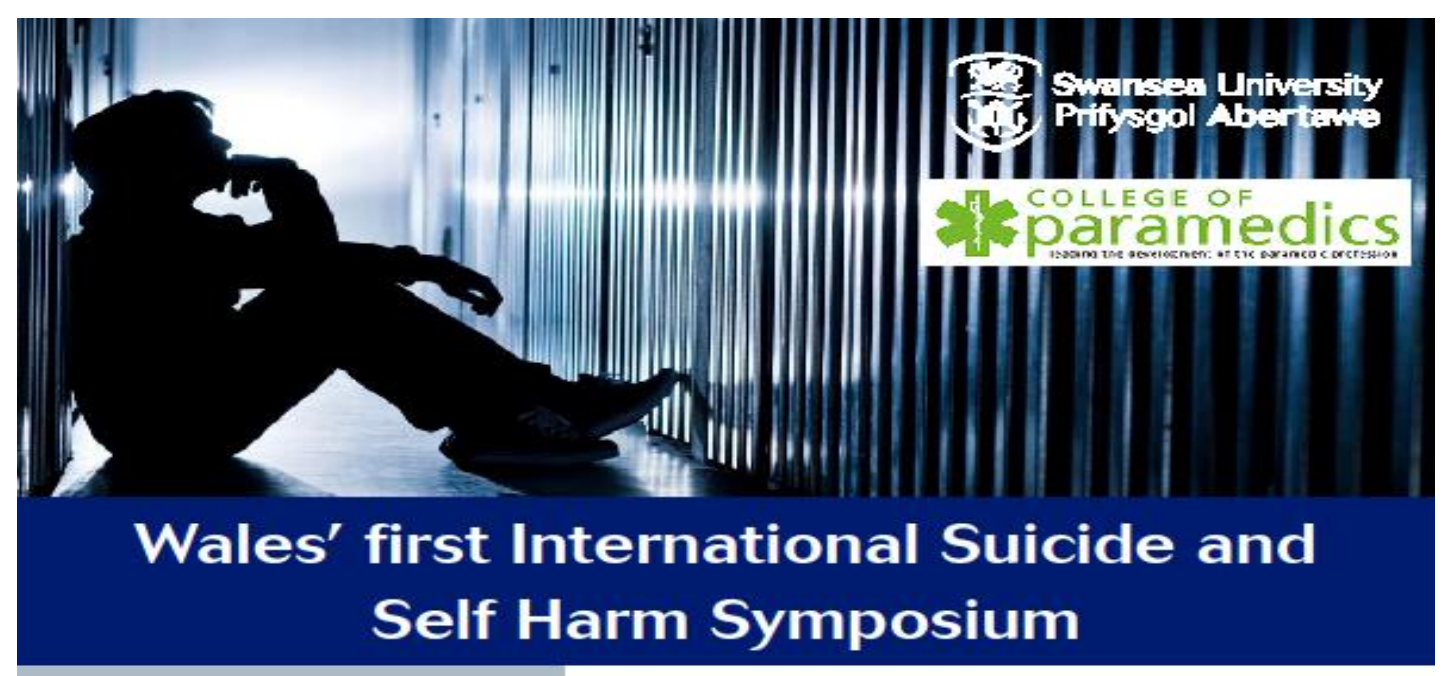

About the event:

This day has been created to help priority care providers (those who respond to people in crisis) prevent suicide and self harm, treat those who have attempted suicide and self harm and care for those who have been affected by these issues. Expert guests will speak and take questions from the audience

Date:

22nd September 2017

Venue:

All Nations Centre

Sachville Avenue, Heath.

Cardiff, CF14 3NY

Costs:

College of Paramedics members $£ 15$ Non-members $£ 30$

Refreshments, lunch and CPD certificates will be provided.

Places are limited to 180 Book your place today by visiting: wrww.collegeofparamedics.co.uk/events

All welcome!

Event schedule:

08:30am Registration. Tea/Coffee

09:00am Welcome

09:05am Professor Ella Arensman - President of the International Association for Suicide Prevention.

09:50am Professor Dr. Ann John - Welsh National lead for suicide and self harm prevention.

10:35am Break tea/coffee

11:00am Mr Ged Flynn - Chief Executive of Papyrus. Prevention of young suicide.

11:45am Detective Chief Superintendent Paul Hurley - Effective

12:30pm Panel Questions

$12: 45 \mathrm{pm}$ Lunch 01:30pm Dr Ben Windsor-Shellard - Senior Research Officer. Office
of National Statistics. Suicide by occupation

02:00pm Mr David Davis - National Clinical Lead / Head of Programme Integrated Urgent Care (NHS 111) Workforce Development

02:30pm Break. Tea/Coffee

03:00pm Mr Nigel Rees - Head of research and innovation at Welsh Ambulance Service. Perceptions of self-harm.

03:30pm Mr Neil Hore - Swansea University Paramedic Sciences Lead. College of Paramedics National Education Advisor. Th education of suicide and self harm prevention.

04:00pm Ms Sarah Stone - Executive Director for Wales, Samaritans.

04.30pm Panel Questions \& Event close 


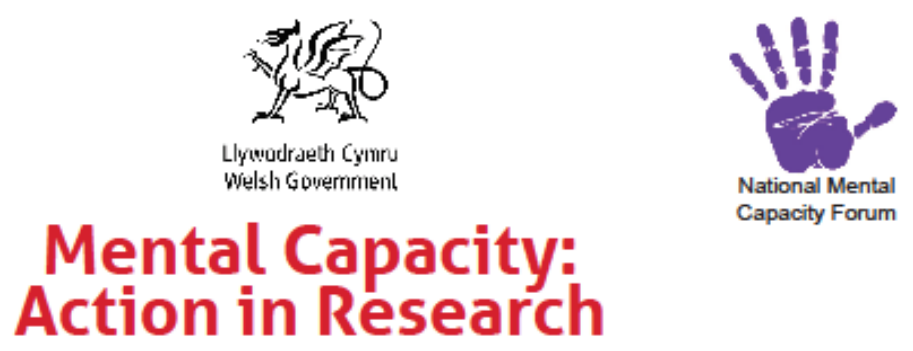

16 March 2016

Ferrier Hall, City Hall, Cardiff

10:00 Registration $\theta$ refreshments

10:30 Welcome from Chair

Dr Sarah Watkins, Head of Mental Health and Vulnerable Groups Policy. Welsh Government

10:40 Keynote

Baroness Finlay of Llandaff, Chair National Mental Capacity Forum

10:50 Personal perspectives

Legal - Heledd Wyn, Solicitor, Estate Planning, Care \& Capacity, Mowbray Woodwards

Researcher - Victoria Shepherd, Research Associate, Cardiff University

Participant - Charlotte Harding, research champion, National Centre for Mental Health

Carer - Mrs Caryl Kelly, Carers Wales

11:10 Issues in practice workshops

- Emergency care - Nigel Rees, Head of Research $\&$ Innovation, Welsh Ambulance Services NHS Trust

- Intensive care - Dr Matt Wise, Critical Care Consultant

- Learning disability - Prof Ruth Northway, Professor of Learning Disability Nursing, University of South Wales

- Dementias - Prof Bob Woods, Director, Dementia Services Development Centre, Bangor University

- Mental health - Prof lan Jones, Director, National Centre for Mental Health

- End of life / palliative - Dr Anthony Byrne, Clinical Director, Marie Curie Palliative Care Research Centre

\section{2:10 Lunch}

13:15 Keynote - William Mieville-Hawkins

13:45 Issues in practice workshops

- Emergency care - Nigel Rees, Head of Research $\theta$ Innovation, Welsh Ambulance Services NHS Trust

- Intensive care - Dr Matt Wise, Critical Care Consultant

- Learning disability - Prof Ruth Northway, Professor of Learning Disability Nursing, University of South Wales

- Dementias - Prof Bob Woods, Director, Dementia Services Development Centre, Bangor University

- Mental health - Prof lan Jones, Director, National Centre for Mental Health

- End of life / palliative - Dr Anthony Byrne, Clinical Director, Marie Curie Palliative Care Research Centre

15:00 Vaughan Gething, Cabinet Secretary for Health, Well-being and Sport

15:10 Make a difference: next actions

Chaired by Baroness Finlay of Llandaff, Chair National Mental Capacity Forum

\section{\#MCresearch}




\section{Euro Global Summit and Medicare Expo on Psychiatry}

July 20-22, 2015 Barcelona, Spai

To explore paramedics perceptions and experiences of caring for people who Self $\mathrm{Harm}(\mathrm{SH})$ in order to inform education and policy

Nioel Rece

Woleh Amb-lanco Sorvioge NHS Truat, UK

Background: The U.K. has one of the highest rates of SH in Europe. SH is amongst the five top causes of acute hospital admissions. Paramedics are often the first professionals encountered following $\mathrm{SH}_{\text {, yet few have investigated their care }}$

Methods: The theoretical perspectivedraws on Evolved Grounded Thecry. Systematic reviews of quantitative and metasynthes is of qualitative literature were conducted. Fifteen paramedics particlpated semi structured interviews, generating themes by constant comparison, open, axial and selective coding

Results: Systematic review identified themes: Effect of SH education and training age of staff, gender, attitudes towards SH, setting anfluence of policies and guidelines. Metasynthesis revealed metaphors. Frustration, futlity and legitimacy of care. First contact in the pre hospital environment: Talking immediate and lasting implications of the moral agent. Decislon making in SH balancing legislation, risk and autowomy. Paramedics' perceptions: harnessing professlonallsm and opportunities to contribute to the care of SH. Paramedicine: Culture and education in transition.

Themes emerging from interviews induding professionalism, legislation and ethical issues.

The therwe Relationships with Police: Procedural, historical practices and evolution of surreptitious strategles.

sif you wanted somebody detalned then we all know detertion under Section 136 needs to take place in a public place. Therefore often the easiest way to do it, to prevent somebody, or to treat sorwebody with self harm was normally to try to coax thern into public place where a pollce officer could take action, because they were, obvioushy very reluctant to do it in a private property:"

Conclusion: Paramediccare for SH is a complex professional, clinical and sodal interaction, the developing theoryis continuing to evolve our understanding of this encounter, and potential contribution of paramedics to SH care. 


\section{Appendix O}

\section{Authorship statement}

\section{Declaration:}

Three chapters in this thesis were associated with published papers but did not reproduce them exactly. These chapters include the systematic review of quantitative literature (Rees et al 2014) in chapters four, the Metasynthesis of qualitative literature (Rees et al 20015) in chapter five, and results (Rees et al 2018) in chapter in six. The following people and institutions contributed to these publications:

\begin{tabular}{|l|l|}
\hline Candidate & $\begin{array}{l}\text { Nigel Rees } \\
\text { Swansea University Medical School }\end{array}$ \\
\hline Author 1 & $\begin{array}{l}\text { Associate Professor Alison Porter } \\
\text { Institute of Life Sciences, Swansea University } \\
\text { Medical School, Swansea, Wales, United Kingdom }\end{array}$ \\
\hline Author 2 & $\begin{array}{l}\text { Professor Frances Rapport } \\
\text { Australian Institute of Health Innovation, Macquarie } \\
\text { University, Sydney, New South Wales, Australia }\end{array}$ \\
\hline Author $\mathbf{3}$ & $\begin{array}{l}\text { Professor Ann John } \\
\text { Farr Institute, Swansea University Medical School, } \\
\text { Swansea, Wales, United Kingdom }\end{array}$ \\
\hline Author $\mathbf{4}$ & $\begin{array}{l}\text { Professor Helen Snooks } \\
\text { Institute of Life Sciences, Swansea University, } \\
\text { Singleton Park, Swansea, Wales SA2 8PP, United } \\
\text { Kingdom. }\end{array}$ \\
\hline Author 5 & $\begin{array}{l}\text { Sarah Hughes } \\
\text { Abertawe Bro Morgannwg University Health Board, } \\
\text { Princess of Wales Hospital, Bridgend, Wales, United } \\
\text { Kingdom }\end{array}$ \\
\hline Author $\mathbf{6}$ & $\begin{array}{l}\text { Gareth Thomas } \\
\text { Institute of Life Sciences, Swansea University, } \\
\text { Singleton Park, Swansea, Wales SA2 8PP, United } \\
\text { Kingdom. }\end{array}$ \\
\hline \hline
\end{tabular}

\section{Author details and their roles:}

\section{Paper 1}

Nigel Rees, Alison Porter, Frances Rapport, Sarah Hughes, Ann John (2018) Paramedics' perceptions of the care they provide to people who self-harm: A qualitative study using evolved grounded theory methodology. Plos One Published: October 17, 2018 https://doi.org/10.1371/journal.pone.0205813 
Located in: Chapter Six

Candidate contributed to:

Conceptualization, Data curation, Formal analysis, Funding acquisition, Investigation, Methodology, Project administration, Resources, Validation, Visualization, Writing - original draft, Writing - review \& editing

Author 1 contributed to: Formal analysis, Methodology, Writing - original draft Writing - review \& editing Supervision,

Author 2 contributed Formal analysis, Methodology, Writing - original draft, Writing - review \& editing Supervision,

Author 3 contributed Formal analysis, Methodology, Writing - original draft, Writing - review \& editing Supervision,

Author 4 Acknowledgement for discussions held.

Author 5 contributed to: contributed to: Validation, Writing - review \& editing

\section{Paper 2}

Nigel Rees, Frances Rapport, Helen Snooks (2015) Perceptions of paramedics and emergency staff about the care they provide to people who self-harm:

Constructivist metasynthesis of the qualitative literature. Journal of

Psychosomatic Research. Volume 78, Issue 6, June 2015, Pages 529-535

Located in: Chapter Six

Candidate contributed to:

Conceptualization, Data curation, Formal analysis, Funding acquisition, Investigation, Methodology, Project administration, Resources, Validation, Visualization, Writing - original draft, Writing - review \& editing

Author 2 contributed to: Formal analysis, Methodology, Writing - original draft Writing - review \& editing Supervision,

Author 4 contributed Formal analysis, Methodology, Writing - original draft, Writing - review \& editing Supervision,

\section{Paper 3}

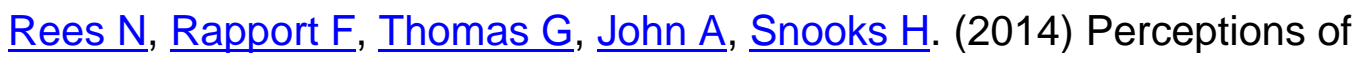
paramedic and emergency care workers of those who self harm: a systematic review of the quantitative literature. Journal of Psychosomtic Research. 2014 Dec;77(6):449-56. doi: 10.1016/j.jpsychores.2014.09.006. Epub 2014 Sep 16.

Located in: Chapter Six

\section{Candidate contributed to:}

Conceptualization, Data curation, Formal analysis, Funding acquisition, Investigation, Methodology, Project administration, Resources, Validation, Visualization, Writing - original draft, Writing - review \& editing

Author 2 contributed to: Formal analysis, Methodology, Writing - original draft Writing - review \& editing Supervision,

Author 3 contributed Formal analysis, Methodology, Writing - original draft, Writing - review \& editing Supervision

Author 4 contributed Formal analysis, Methodology, Writing - original draft, Writing - review \& editing Supervision,

Author 6 contributed to: contributed to: Validation, Writing - review \& editing 
We the undersigned agree with the above stated "proportion of work undertaken" for each of the above published peer-reviewed manuscripts contributing to this thesis:

Signed Candidate

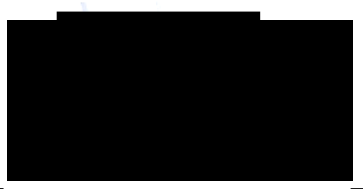

Author 1

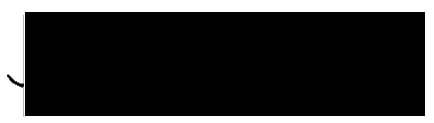

Author 2

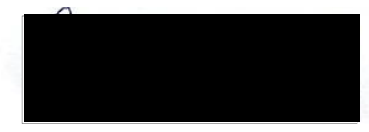

Author 3

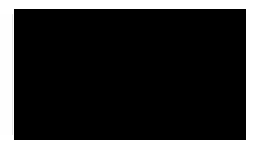

Author 4

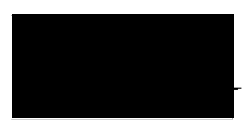

Author 5

Author $6 \quad$ Unable to contact 